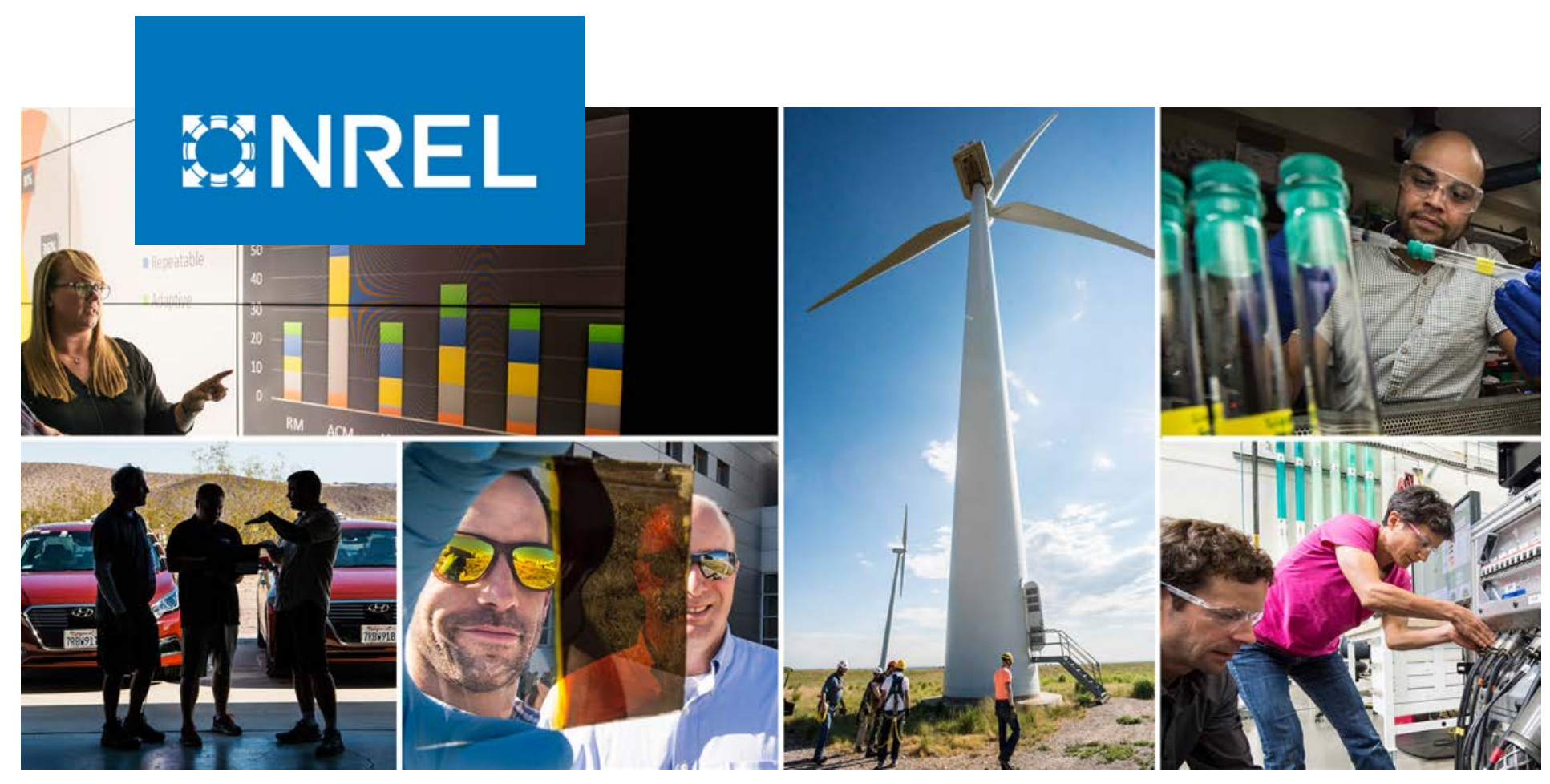

\title{
MOVES Activity Updates Using Fleet DNA Data: Interim Report
}

Andrew Kotz and Kenneth Kelly

National Renewable Energy Laboratory

Produced under direction of the U.S. Environmental Protection Agency by the National Renewable Energy Laboratory (NREL) under Interagency Agreement 92402501.

NREL is a national laboratory of the U.S. Department of Energy Office of Energy Efficiency \& Renewable Energy

Operated by the Alliance for Sustainable Energy, LLC

This report is available at no cost from the National Renewable Energy Laboratory (NREL) at www.nrel.gov/publications.
Strategic Partnership Project Report NREL/TP-5400-70671

January 2019 


\title{
FANEL
}

\section{MOVES Activity Updates Using Fleet DNA Data: Interim Report}

\author{
Andrew Kotz and Kenneth Kelly
}

National Renewable Energy Laboratory

\section{Suggested Citation}

Kotz, Andrew and Kenneth Kelly. 2019. MOVES Activity Updates Using Fleet DNA Data:

Interim Report. Golden, CO: National Renewable Energy Laboratory.

NREL/TP-5400-70671. https://www.nrel.gov/docs/fy19osti/70671.pdf.

NREL is a national laboratory of the U.S. Department of Energy Office of Energy Efficiency \& Renewable Energy Operated by the Alliance for Sustainable Energy, LLC

This report is available at no cost from the National Renewable Energy Laboratory (NREL) at www.nrel.gov/publications.

Contract No. DE-AC36-08GO28308
Strategic Partnership Project Report NREL/TP-5400-70671 January 2019

National Renewable Energy Laboratory 15013 Denver West Parkway Golden, CO 80401 303-275-3000 • www.nrel.gov 


\section{NOTICE}

This work was supported by the National Renewable Energy Laboratory, operated by Alliance for Sustainable Energy, LLC, for the U.S. Department of Energy (DOE) under Contract No. DE-AC36-08GO28308, and U.S. Environmental Protection Agency under Interagency Agreement 92402501. The views expressed herein do not necessarily represent the views of the DOE or the U.S. Government.

This report is available at no cost from the National Renewable Energy Laboratory (NREL) at www.nrel.gov/publications.

U.S. Department of Energy (DOE) reports produced after 1991 and a growing number of pre-1991 documents are available free via www.OSTI.gov.

Cover Photos by Dennis Schroeder: (clockwise, left to right) NREL 51934, NREL 45897, NREL 42160, NREL 45891, NREL 48097, NREL 46526.

NREL prints on paper that contains recycled content. 


\section{Acknowledgments}

The authors would like to thank Darrell Sonntag, Gary Dolce, David Brzezinski, Jessica Brakora, and Carl Fulper of the U.S. Environmental Protection Agency (EPA) as well as Sudheer Ballare, an Oak Ridge Institute for Science and Education participant supported by an interagency agreement between the EPA and the U.S. Department of Energy, for their assistance with creating this report. 


\section{Table of Contents}

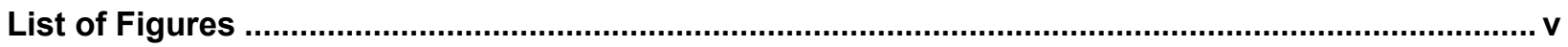

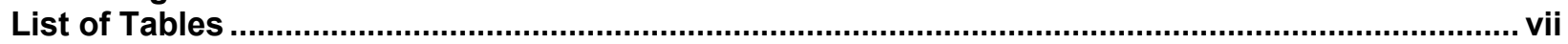

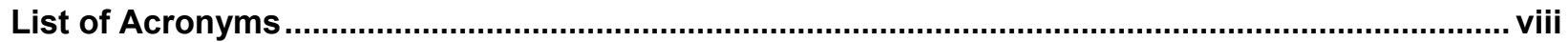

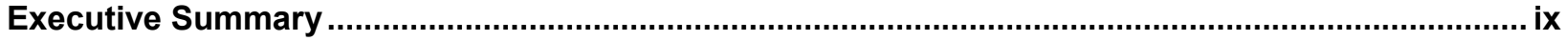

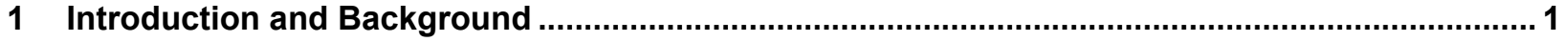

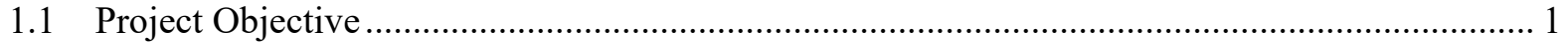

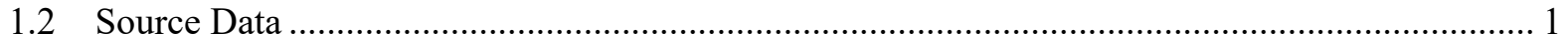

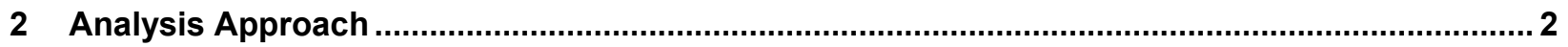

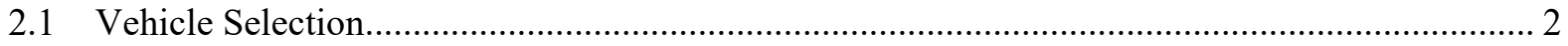

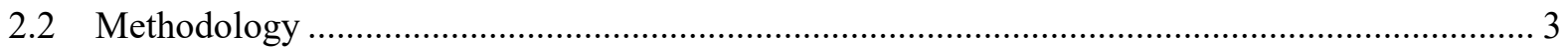

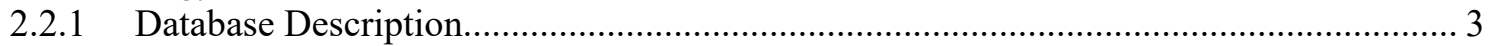

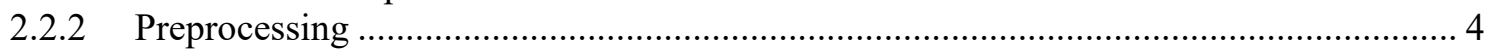

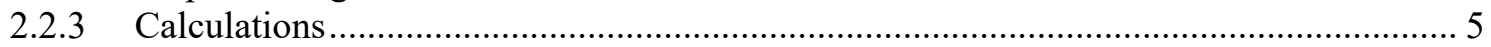

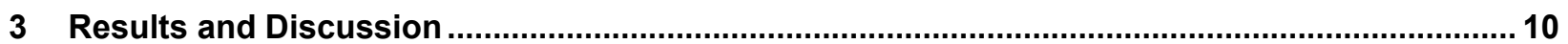

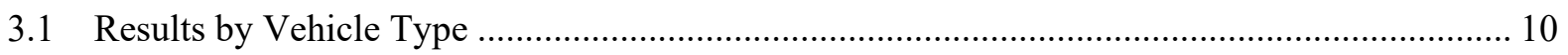

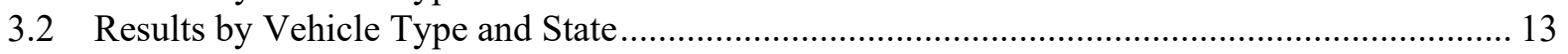

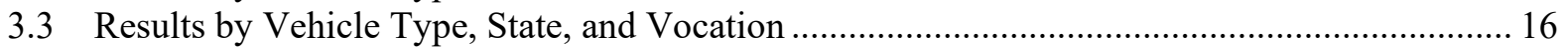

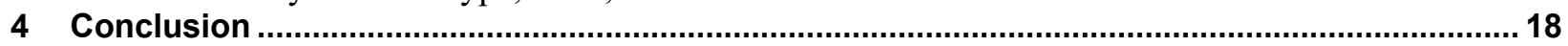

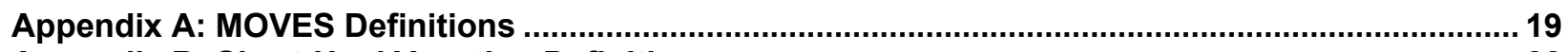

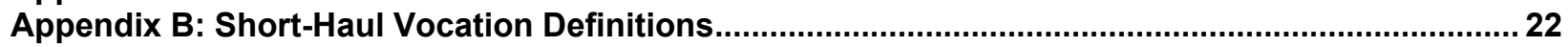

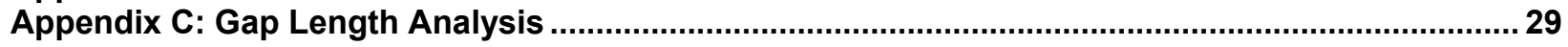

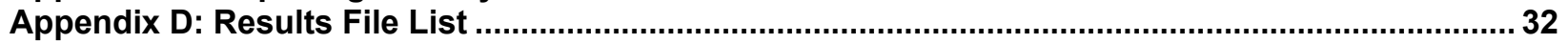

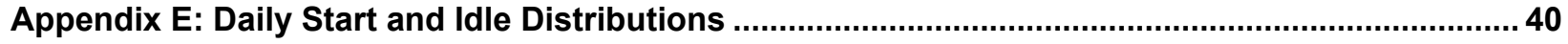

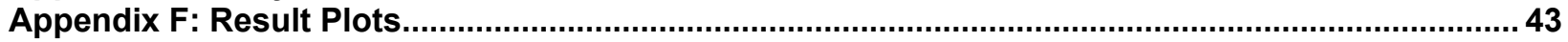

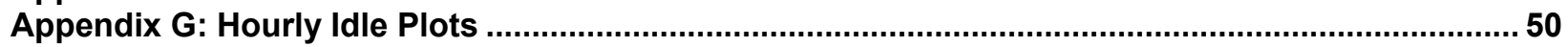

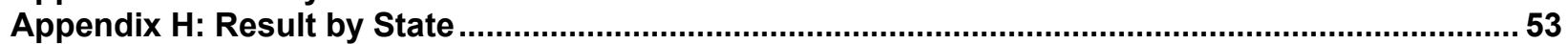

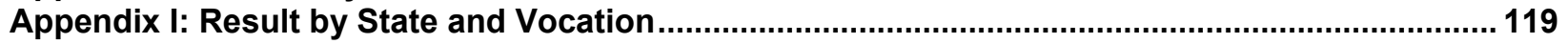

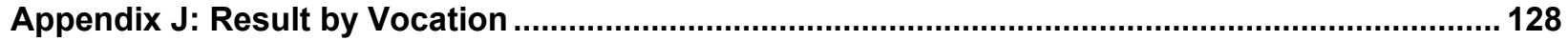

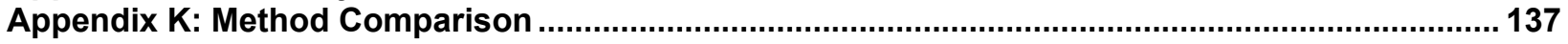




\section{List of Figures}

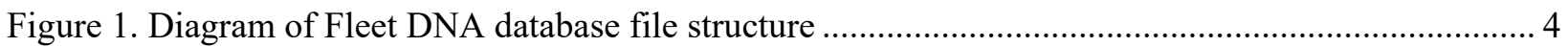

Figure 2. Soak fraction multiplied by start fraction vs. hour of the day for weekdays ............................. 11

Figure 3. Extended and workday idle fractions vs. day type and vehicle type ........................................ 12

Figure 4. Extended and workday idle fractions vs. vehicle type for weekdays ....................................... 13

Figure 5. Start fraction times normalized soak fraction vs. hour for short-haul classes ........................... 14

Figure 6. Vocational differences between beverage and parcel delivery combination trucks ................... 16

Figure 7. Start and soak distributions for single-unit California delivery vehicles................................ 17

Figure C.1. Start fraction weighted soak distribution by gap length: Comb.-Unit Long-Haul................... 30

Figure C.2. Start fraction weighted soak distribution by gap length: Single-Unit Short-Haul .................. 31

Figure F.1. Soak fraction and start fraction vs. hour of the day for Transit Bus..................................... 43

Figure F.2. Soak fraction and start fraction vs. hour of the day for School Bus....................................... 44

Figure F.3. Soak fraction and start fraction vs. hour of the day for Refuse Truck ................................. 45

Figure F.4. Soak fraction and start fraction vs. hour of the day for Single-Unit Short-Haul.................... 46

Figure F.5. Soak fraction and start fraction vs. hour of the day for Comb.-Unit Short-Haul ..................... 47

Figure F.6. Soak fraction and start fraction vs. hour of the day for Comb.-Unit Long-Haul ..................... 48

Figure F.7. Extended and workday idle fractions vs. day type and vehicle type .................................... 49

Figure G.1. Hourly idle fraction vs. hour of the day for Transit Bus.................................................. 50

Figure G.2. Hourly idle fraction vs. hour of the day for School Bus ...................................................... 50

Figure G.3. Hourly idle fraction vs. hour of the day for Refuse Truck.................................................. 51

Figure G.4. Hourly idle fraction vs. hour of the day for Single-Unit Short-Haul .................................... 51

Figure G.5. Hourly idle fraction vs. hour of the day for Comb.-Unit Short-Haul ................................... 52

Figure G.6. Hourly idle fraction vs. hour of the day for Comb.-Unit Long-Haul ................................... 52

Figure H.1. Soak fraction and start fraction vs. hour of the day for Transit Bus - CO ............................ 53

Figure H.2. Soak fraction and start fraction vs. hour of the day for Transit Bus - MN ........................... 54

Figure H.3. Soak fraction and start fraction vs. hour of the day for Transit Bus - TN .............................55

Figure H.4. Extended and workday idle fractions vs. day type and state for Transit Bus ........................ 56

Figure H.5. Soak fraction and start fraction vs. hour of the day for School Bus - CA ............................5 57

Figure H.6. Soak fraction and start fraction vs. hour of the day for School Bus - Blank ......................... 58

Figure H.7. Extended and workday idle fractions vs. day type and state for School Bus ........................ 59

Figure H.8. Soak fraction and start fraction vs. hour of the day for Refuse Truck - CO ...........................6 60

Figure H.9. Soak fraction and start fraction vs. hour of the day for Refuse Truck - FL .......................... 61

Figure H.10. Soak fraction and start fraction vs. hour of the day for Refuse Truck - IN .......................... 62

Figure H.11. Soak fraction and start fraction vs. hour of the day for Refuse Truck - OH........................ 63

Figure H.12. Soak fraction and start fraction vs. hour of the day for Refuse Truck - Blank ...................... 64

Figure H.13. Extended and workday idle fractions vs. day type and state for Refuse Truck ..................... 65

Figure H.14. Soak fraction and start fraction vs. hour of the day for Single-Unit Short-Haul - CA .......... 66

Figure H.15. Soak fraction and start fraction vs. hour of the day for Single-Unit Short-Haul - CO .......... 67

Figure H.16. Soak fraction and start fraction vs. hour of the day for Single-Unit Short-Haul - IN........... 68

Figure H.17. Soak fraction and start fraction vs. hour of the day for Single-Unit Short-Haul - MN ......... 69

Figure H.18. Soak fraction and start fraction vs. hour of the day for Single-Unit Short-Haul - TN .......... 70

Figure H.19. Soak fraction and start fraction vs. hour of the day for Single-Unit Short-Haul - TX .......... 71

Figure H.20. Soak fraction and start fraction vs. hour of the day for Single-Unit Short-Haul - UT .......... 72

Figure H.21. Soak fraction and start fraction vs. hour of the day for Single-Unit Short-Haul - WA ......... 73

Figure H.22. Soak fraction and start fraction vs. hour of the day for Single-Unit Short-Haul - Blank ...... 74

Figure H.23. Extended and workday idle fractions vs. day type and state for Single-Unit Short-Haul ..... 75

Figure H.24. Soak fraction and start fraction vs. hour of the day for Comb.-Unit Short-Haul - CA.......... 76

Figure H.25. Soak fraction and start fraction vs. hour of the day for Comb.-Unit Short-Haul - CO.......... 77

Figure H.26. Soak fraction and start fraction vs. hour of the day for Comb.-Unit Short-Haul - FL........... 78

Figure H.27. Soak fraction and start fraction vs. hour of the day for Comb.-Unit Short-Haul - MD........ 79 
Figure H.28. Soak fraction and start fraction vs. hour of the day for Comb.-Unit Short-Haul - MI .......... 80

Figure H.29. Soak fraction and start fraction vs. hour of the day for Comb.-Unit Short-Haul - TN......... 81

Figure H.30. Soak fraction and start fraction vs. hour of the day for Comb.-Unit Short-Haul - TX.......... 82

Figure H.31. Soak fraction and start fraction vs. hour of the day for Comb.-Unit Short-Haul - WI .......... 83

Figure H.32. Soak fraction and start fraction vs. hour of the day for Comb.-Unit Short-Haul - Blank...... 84

Figure H.33. Extended and workday idle fractions vs. day type and state for Comb.-Unit Short-Haul..... 85

Figure H.34. Soak fraction and start fraction vs. hour of the day for Comb.-Unit Long-Haul - AL .......... 86

Figure H.35. Soak fraction and start fraction vs. hour of the day for Comb.-Unit Long-Haul - AR .......... 87

Figure H.36. Soak fraction and start fraction vs. hour of the day for Comb.-Unit Long-Haul - AZ .......... 88

Figure H.37. Soak fraction and start fraction vs. hour of the day for Comb.-Unit Long-Haul - CA .......... 89

Figure H.38. Soak fraction and start fraction vs. hour of the day for Comb.-Unit Long-Haul - CO .......... 90

Figure H.39. Soak fraction and start fraction vs. hour of the day for Comb.-Unit Long-Haul - ID ........... 91

Figure H.40. Soak fraction and start fraction vs. hour of the day for Comb.-Unit Long-Haul - IL............ 92

Figure H.41. Soak fraction and start fraction vs. hour of the day for Comb.-Unit Long-Haul - IN ........... 93

Figure H.42. Soak fraction and start fraction vs. hour of the day for Comb.-Unit Long-Haul - KS .......... 94

Figure H.43. Soak fraction and start fraction vs. hour of the day for Comb.-Unit Long-Haul - KY .......... 95

Figure H.44. Soak fraction and start fraction vs. hour of the day for Comb.-Unit Long-Haul - MD ......... 96

Figure H.45. Soak fraction and start fraction vs. hour of the day for Comb.-Unit Long-Haul - MN ......... 97

Figure H.46. Soak fraction and start fraction vs. hour of the day for Comb.-Unit Long-Haul - MO ......... 98

Figure H.47. Soak fraction and start fraction vs. hour of the day for Comb.-Unit Long-Haul - MS.......... 99

Figure H.48. Soak fraction and start fraction vs. hour of the day for Comb.-Unit Long-Haul - MT ....... 100

Figure H.49. Soak fraction and start fraction vs. hour of the day for Comb.-Unit Long-Haul - NC ........ 101

Figure H.50. Soak fraction and start fraction vs. hour of the day for Comb.-Unit Long-Haul - ND........ 102

Figure H.51. Soak fraction and start fraction vs. hour of the day for Comb.-Unit Long-Haul - NE ........ 103

Figure H.52. Soak fraction and start fraction vs. hour of the day for Comb.-Unit Long-Haul - NM ....... 104

Figure H.53. Soak fraction and start fraction vs. hour of the day for Comb.-Unit Long-Haul - NV ........ 105

Figure H.54. Soak fraction and start fraction vs. hour of the day for Comb.-Unit Long-Haul - NY........ 106

Figure H.55. Soak fraction and start fraction vs. hour of the day for Comb.-Unit Long-Haul - OH........ 107

Figure H.56. Soak fraction and start fraction vs. hour of the day for Comb.-Unit Long-Haul - OK........ 108

Figure H.57. Soak fraction and start fraction vs. hour of the day for Comb.-Unit Long-Haul - OR ........ 109

Figure H.58. Soak fraction and start fraction vs. hour of the day for Comb.-Unit Long-Haul - PA ........ 110

Figure H.59. Soak fraction and start fraction vs. hour of the day for Comb.-Unit Long-Haul - SC......... 111

Figure H.60. Soak fraction and start fraction vs. hour of the day for Comb.-Unit Long-Haul - SD ........ 112

Figure H.61. Soak fraction and start fraction vs. hour of the day for Comb.-Unit Long-Haul - TN ........ 113

Figure H.62. Soak fraction and start fraction vs. hour of the day for Comb.-Unit Long-Haul - TX ........ 114

Figure H.63. Soak fraction and start fraction vs. hour of the day for Comb.-Unit Long-Haul - UT ........ 115

Figure H.64. Soak fraction and start fraction vs. hour of the day for Comb.-Unit Long-Haul - WA....... 116

Figure H.65. Soak fraction and start fraction vs. hour of the day for Comb.-Unit Long-Haul - WY ....... 117

Figure H.66. Extended and workday idle fractions vs. day type and state for Comb.-Unit Long-Haul. Note the Maryland Idle Fraction is based on only one truck. 118 


\section{List of Tables}

Table 1. Heavy-Duty Vehicle Types with Population of Conventional Vehicles in Fleet DNA ................. 2

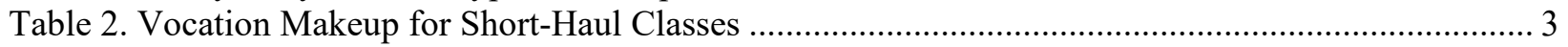

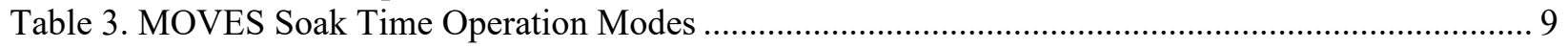

Table 4. Summary Statistics: Weekday Averages by Vehicle Type...................................................... 10

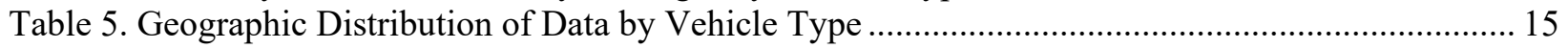

Table 6. Average Daily Distance by Vocation for Short-Haul Vehicle Types ........................................ 16

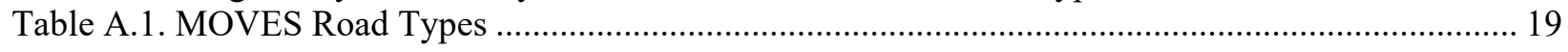

Table A.2. Heavy-Duty Vehicle MOVES Vehicle Types .................................................................... 19

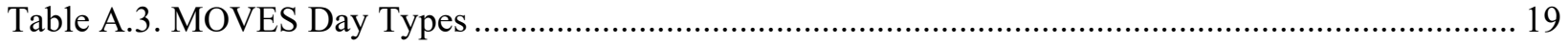

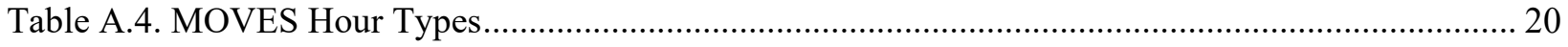

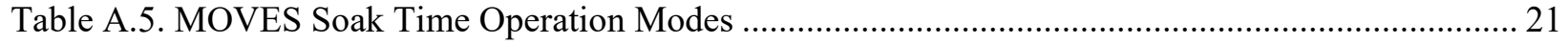




\section{List of Acronyms}

EPA U.S. Environmental Protection Agency

GPS

MOVES

$\mathrm{mph}$

NREL

rpm

Global Positioning System

Motor Vehicle Emissions Simulator

miles per hour

National Renewable Energy Laboratory

revolutions per minute 


\section{Executive Summary}

The U.S. Environmental Protection Agency's (EPA's) MOtor Vehicle Emissions Simulator (MOVES) is a publicly available tool used by researchers and policy makers to help understand motor vehicle emission sources at a national, county, and project level. Estimates of heavy-duty activity in the most recent version of the model, MOVES2014, have been identified as an area needing improvement. The start activity in MOVES2014 is based on a limited and dated data set. In addition, MOVES2014 relies on drive cycles that represent on-network activity, but does not account for idling activity that occurs on off-network roads, such as at a distribution center, while the truck is queuing or during loading and unloading. As a result, MOVES may currently underestimate the number of starts, and idle and soak time for heavy-duty trucks in real-world operation.

Under the guidance and expertise of the EPA, the National Renewable Energy Laboratory (NREL) has leveraged its expansive Fleet DNA database of heavy-duty vehicles to reinforce the data set behind the next-generation MOVES model and enhance idle and start activity for six of the nine heavy-duty vehicle classes in the model. The data available in Fleet DNA from 415 conventional, diesel-powered vehicles provided activity estimates from more than 120,000 hours of operation throughout 14,682 vehicle days between October 2006 and January 2016. NREL calculated start fraction, starts per day, soak fraction, and idle fraction by hour of the day for each vehicle type, state, and vocation, and provided results in .CSV files that can be translated to MOVES table inputs. This midterm report details these results providing graphical analysis and context for the start, soak, and idle distributions. 


\section{Introduction and Background}

\subsection{Project Objective}

The U.S. Environmental Protection Agency's (EPA's) MOtor Vehicle Emissions Simulator (MOVES) is a publicly available tool used by researchers and policy makers to help understand motor vehicle emission sources at a national, county, and project level. However, current estimates of heavy-duty activity are based on a limited and dated data set ${ }^{1}$. Further, there is concern that emissions from off-network activity are not accounted for with existing on-network activity. Under the guidance and expertise of the EPA, the National Renewable Energy Laboratory (NREL) has leveraged its extensive Fleet DNA database of heavy-duty vehicles to reinforce the data set behind the next-generation MOVES model and enhance vehicle idle rates, start activity, and soak fractions for six of the nine heavy-duty vehicle classes in MOVES. Definitions of these activity types are available in section 2.2.3.

\subsection{Source Data}

In collaboration with fleet and industry partners across the country, the Commercial Vehicle Technologies team in NREL's Transportation and Hydrogen Systems Center focuses on evaluating the real-world performance of alternative fuel and advanced vehicle technologies deployed in medium- and heavy-duty commercial fleet vehicles throughout the United States. NREL's Commercial Vehicle Technologies team instruments vehicles operating in the field with data recording devices to capture $1-\mathrm{Hz}$ telematics and controller area network data and works with industry partners to collect existing vehicle data sets. The data are then used to calculate inuse fuel economy and perform drive-cycle characterization and system-level duty-cycle analysis, which can be complemented with chassis dynamometer emissions and fuel economy testing. These results are used to provide feedback to stakeholders such as fleets, technology providers, researchers, and government agencies, helping to inform and provide insight on the real-world performance of advanced technology and fuels. The data for these evaluation projects are stored in the Fleet DNA database along with additional externally-sourced data that have been supplied by Fleet DNA project partners. Data from 415 conventional, diesel-powered vehicles was used to provide activity estimates from more than 120,000 hours of operation throughout 14,682 vehicle days between October 2006 and January 2016.

\footnotetext{
${ }^{1}$ The starts and soak lengths in MOVES2014 were derived from datasets that tested 124 trucks in late 1990's and early 2000s's. The MOVES inputs for most of the heavy-duty source types were estimated using less than 200 vehicle trips from these trucks per source type. See USEPA (2016). Population and Activity of On-road Vehicles in MOVES2014. EPA-420-R-16-003a. Ann Arbor, MI, Office of Transportation and Air Quality. US Environmental Protection Agency. March 2016. https://www.epa.gov/moves/moves-technical-reports.
} 


\section{Analysis Approach}

This report provides an analysis of heavy-duty idle rates, start activity, and soak fractions in accordance with the EPA's direction. Activity data in the default MOVES database is differentiated according to road type, vehicle type, day type of either weekday or weekend, and hour of the day. The formal definitions of these distinctions are provided in Appendix A. The following sections provide the methodology of NREL's analysis.

\subsection{Vehicle Selection}

While the Fleet DNA database includes a wide range of vehicle drivetrains and propulsion mechanisms, a more limited selection containing diesel-powered conventional vehicles was identified for this study to ensure the selected drive cycles are representative of traditional operation and not modified to accommodate vehicle architecture. Additionally, Fleet DNA vehicles with missing or erroneous engine data were filtered out. The final statistics in this report are derived from the population of conventional vehicles shown in Table 1 and Table 2.

Table 1. Heavy-Duty Vehicle Types with Population of Conventional Vehicles in Fleet DNA

\begin{tabular}{|llll|}
\hline MOVES Vehicle ID & Vehicle Type & Vehicles in Fleet DNA & Days of Data \\
\hline 41 & Intercity Buses (non-school / transit) & 0 & 0 \\
\hline 42 & Transit Buses & 16 & 810 \\
\hline 43 & School Buses & 7 & 246 \\
\hline 51 & Refuse Trucks & 37 & 641 \\
\hline 52 & Single-Unit Short-Haul Trucks & 119 & 2,794 \\
\hline 53 & Single-Unit Long-Haul Trucks & 0 & 0 \\
\hline 54 & Motor Homes & 0 & 0 \\
\hline 61 & Combination-Unit Short-Haul Trucks & 105 & 2,631 \\
\hline 62 & Combination-Unit Long-Haul Trucks & 131 & 7,560 \\
\hline & Total & $\mathbf{4 1 5}$ & $\mathbf{1 4 , 6 8 2}$ \\
\hline
\end{tabular}

The vehicle classifications shown in Table 1 are the vehicle types provided by the EPA that correspond to the MOVES inputs. The number of NREL's Fleet DNA vehicle data sets matching those classifications is shown in the far-right column. The vehicle vocation composition for the two short-haul classes is provided in Table 2. Specific definitions are provided in Appendix B to give an understanding of the population diversity for these broad classes. The vehicle vocations shown in Table 2 were self-reported by the collecting agency making the vocational makeup and subsequent differentiation between short- and long-haul vocations slightly subjective. To classify single- and combination-unit vehicles as short or long-haul, we used the daily mean trip length from each truck vehicle grouping from each data provider (e.g., combination drayage trucks from NREL's Fleet DNA) as an objective measure. If most of the vehicles in a truck group drove an average of 200 or more miles per operating day, the trucks were considered long-haul trucks. Conversely, if most of the vehicles drove less than 200 miles per operating day, the trucks were considered short haul. This objective measure was not used if enough information was known 
about the operation of a vehicle group to classify it differently. For instance, the six parcel delivery tractors from Texas, which are shown in Figure H.30 of Appendix H, had two shifts of drivers operating local pickup and delivery during the day and inter-warehouse delivery at night with each truck returning to the base depot at night. On average, these vehicles drove over 200 miles a day, but because most was local operation, these were classified as short-haul combination-unit trucks.

Table 2. Vocation Makeup for Short-Haul Classes

\begin{tabular}{|llll|}
\hline Comb.-Unit Short-Haul & Number & Single-Unit Short-Haul & Number \\
\hline Beverage Delivery & 10 & Warehouse Delivery & 9 \\
\hline Food Delivery & 13 & Parcel Delivery & 39 \\
\hline Drayage & 28 & Linen Delivery & 17 \\
\hline Transfer Truck & 28 & Food Delivery & 30 \\
\hline Local Delivery & 7 & Snow Plow & 11 \\
\hline Regional Haul & 7 & Towing & 4 \\
\hline Dump Truck & 4 & Concrete & 3 \\
\hline Parcel Delivery & 5 & Delivery & 1 \\
\hline Dry Van & 3 & Shredder & 1 \\
\hline & & Propane Tank & 1 \\
\hline
\end{tabular}

a The concrete trucks in the Fleet DNA database were combination (as shown in Appendix B). For activity purposes, these were grouped with the single-unit short-haul ones because the short-haul combination trucks in MOVES imply delivery trucks, not vocational trucks like concrete mixers.

No single-unit trucks were found to travel more than 200 miles on average, except the $2 \%$ of the single-unit short haul trucks which had additional information regarding operation to identify them as single-unit short haul. Additionally, no information was available that would suggest the trucks traveling less than 200 miles per day on average should be reclassified as long-haul trucks.

This classification method resulted in combination-unit long-haul trucks having $88 \%$ of the vehicles driving over 200 miles on average, $78 \%$ of combination-unit short-haul vehicles driving under 200 miles on average, $98 \%$ of single-unit short-haul vehicles driving under 200 miles on average, and no vehicles being categorized as single-unit long-haul.

\subsection{Methodology}

\subsubsection{Database Description}

Prior to calculation and preprocessing, the data are collected from the database, which involves loading and combining all 1-Hz data from Fleet DNA into a single one-dimensional data array for each parameter. Each data file is arranged in the database by vehicle, day, and parameter as shown in Figure 1. To create one contiguous array per parameter, the processing script loads each parameter and appends it to the parameter from the previous day, resulting in five onedimensional arrays of equal n-length, allowing the five arrays to be joined together creating a single two-dimensional array of n-rows and five columns. 


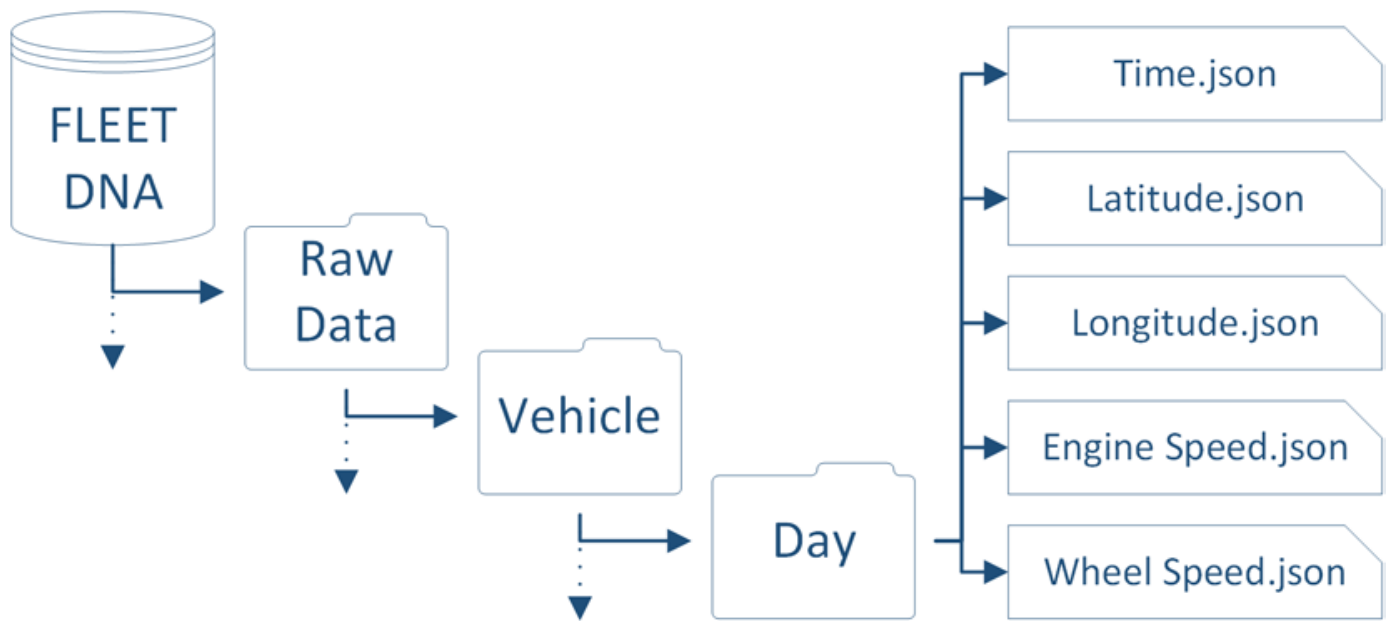

Figure 1. Diagram of Fleet DNA database file structure

\subsubsection{Preprocessing}

Two key parameters in the database are engine speed (revolutions per minute [rpm]) and wheel speed (miles per hour [mph]). An engine speed greater than zero indicates that the vehicle engine is running, and a wheel speed greater than zero mph signifies that the vehicle is in motion. In this analysis, vehicle starts are calculated by identifying the transition from an engine speed of zero to greater than zero, vehicle soak is defined as the length of time between engine off (engine speed of zero) and the next time it is started (engine speed greater than zero), and a vehicle is considered to be idling when its wheel speed is less than one mph and the engine speed is greater than zero.

These calculations depend on accurate reporting of the vehicle data, so data preprocessing is performed to eliminate erroneous information. The first preprocessing step is to filter-out parameter values that are unrealistic. Some vehicle networks will report a meaningless default value (usually this is the "FF" or highest value in hex notation) prior to powering down, which causes the engine speed to falsely exceed 8,000 rpm after the engine shuts off and could then be mischaracterized as a new start. The preprocessing filter removes data points with engine speeds greater than 7,000 rpm to account for this issue. On top of these intrinsic idiosyncrasies, malfunctions and defects can further hinder data quality. For instance, if the wheel speed sensor is broken or not properly installed, it may still report values of zero, causing the vehicle to seem as though it is idling throughout the entire operation of the vehicle. Therefore, in the preprocessing process, if the wheel speed is reported as zero for the entire vehicle operation, we assume a wheel sensor error and use speed from the global positioning system (GPS) as a substitute for wheel speed.

Another common vehicle data characteristic is jumps or "gaps" in timestamp between data points. It is reasonable to expect that a vehicle may slow to zero, shut down the engine, and soak for long periods, such that the next timestamp recorded is an hour or more from the previous timestamp. Alternatively, the GPS may cause a gap in time (generally an hour) when it updates the data logger's clock as the vehicle crosses a time zone, or a gap in time may be generated by the data logger if it pauses the recording while saving a log file to memory. Furthermore, if the logger was taken off a vehicle on the West Coast and placed on a vehicle on the East Coast, it is possible for the timestamp to jump three hours at the beginning of logging once the GPS updates 
the internal clock to local time. These are trivial instances in which there is a logical explanation for a gap in time with near-contiguous data that are valuable to keep. However, in some instances a data logger will shut off prior to recording a zero for engine speed, and it is unclear from the data if the engine turned off. As a result, a corresponding soak time and start may not be accurately categorized or missed altogether. This is addressed during the preprocessing as described below.

The next step of the data preprocessing uses a gap filling algorithm to try and infer engine stops and starts that may have been missed in these nontrivial events by identifying leaps or gaps in timestamp from one data point to the next. If the algorithm finds a time gap larger than a predetermined value of gap length, a zero is added to the start and end of the engine speed data to trigger a shutoff and start determination. When considering an appropriate upper bound for a gap length, we tested gap lengths of 2 seconds, 6 minutes, 30 minutes, 1 hour, 1.5 hours, 2 hours, 6 hours, 12 hours, and 30 hours as defined by the operational mode times for vehicle soak. To prevent counting trivial time gaps as instances where the engine has shut off, we identified 6 hours as an appropriate length of time or "gap length" beyond which we assume an engine-off event has occurred. Appendix $\mathrm{C}$ provides results of this analysis as well as further discussion of the process behind choosing six hours as the designated time gap.

Finally, the first and last days of data are eliminated to avoid counting incomplete or unrepresentative operation when the data logger is being installed or removed. The summary of the preprocessing steps are as follows:

1. Filter-out values that are unrealistic

2. Fill time gaps greater than 6 hours with an engine start

3. Remove the first and last days

\subsubsection{Calculations}

Vehicle activity values in MOVES' default database are intended to represent average activity at a national scale. MOVES uses average idle fraction, start fraction, and soak fraction to quantify off-network activity, and two approaches were considered to translate the Fleet DNA data to national averages.

The first approach calculated idle fractions, start fractions, and soak fractions for individual vehicles and took an average of those fractions from all vehicles in the same vehicle type and day type. This approach created some unrealistically high values for vehicles that had very little operation, because vehicles with low-operation were given equal weight with vehicles with more operation. We do not present the data from this approach in the report, but files are available upon request.

In the second approach, we start by calculating the daily and hourly averages for operation time, idle time, starts, for each individual vehicle. Next, we calculated idle fractions, start fractions, and soak fractions by dividing the sum of the average statistic of interest (e.g daily average idle time) by the denominator of the corresponding statistic (e.g. daily operation time) across all vehicles within the same vehicle and day type. This approach normalizes the recorded activity by 
the amount of time each vehicle was instrumented. We chose to move forward with second approach, which is described in more detail in the following sections, and is used to calculate the results presented in this report. Examples of the impact of calculating the MOVES inputs using the proposed method compared to the first method on the idle fraction, start fraction, and soak fractions is presented in Appendix K.

\subsubsection{Idle Fraction}

Currently in MOVES, off-network activity includes starts, soak, and idling (both extended idle and auxiliary power unit operation for long-haul combination trucks). Activity includes speed distributions as well as the idling that takes place at intersections and on congested roadways. This idling is represented in the MOVES driving cycles that are selected based on the average speed distributions. None of these driving cycles include a full estimate of "workday idle," which also includes the idling that takes place during the "workday" truck operation, such as at distribution centers while the truck is queuing, loading, and unloading. To supplement these gaps in idle activity information, total, workday, and extended idle fractions were calculated for the vehicles provided in Table 1 .

The idle activity analysis provided by NREL (see Appendix D for result files) calculates total idle, workday idle, and extended idle fraction for each vehicle type and day type. However, this is limited to engine idling and does not include additional "hoteling" activity from devices such as auxiliary power units, auxiliary battery power, auxiliary heating units, and other engine-off hoteling operation. After preprocessing, idle is calculated when a vehicle's wheel speed is less than $1 \mathrm{mph}$ and engine speed is greater than $0 \mathrm{rpm}$. Using these two logic statements, a binary output indicates if a vehicle is idling or not. Next, periods of contiguous idle are identified by the length and the day type corresponding to the start of the idle. If an idle period starts during one day type and ends on another, that activity will only be counted for the day type in which it started. For this analysis, operating time is defined as the total time when engine speed is greater than zero.

To calculate the idle fraction for an individual vehicle and specific day type (weekday or weekend), the sum of the idle time is divided by the sum of operation taking place within each day type for each individual vehicle, using Equation 1.

$$
\text { Idle Fraction }=\frac{\sum \text { Idle Time }}{\sum \text { Operating Time }}
$$

To accommodate the request for workday and extended idle fractions, the idle segments were further classified into segments less than or equal to 3,600 seconds (one hour) as workday idle and those greater than 3,600 seconds as extended idle. The workday and extended idle fractions were then calculated using Equations 2 and 3, respectively, such that the sum of the workday and extended idle is equal to the total idle as shown in Equation 4 for each day type and individual vehicle.

$$
\text { Workday Idle Fraction }=\frac{\sum \text { Workday Idle Time }}{\sum \text { operating Time }}
$$




$$
\begin{aligned}
& \text { Extended Idle Fraction }=\frac{\sum \text { Extended Idle Time }}{\sum \text { Operating Time }} \\
& \text { Workday Idle Fraction }+ \text { Extended Idle Fraction }=\text { Idle Fraction }
\end{aligned}
$$

As discussed earlier, two approaches were used to calculate the average idle fraction for a given day type and vehicle type. In the first approach, we averaged idle fraction from Equations 1, 2 and 3 across all vehicles within a vehicle type and day type.

In the second approach, the sum of the average idle time for each vehicle within a day and vehicle type is divided by the sum of the average operation time within each day and vehicle type. Equation 5 provides the calculation of the average idle fraction for each day and vehicle type. We refer to the idle fraction calculated using this approach as idle fraction_final in the data files.

$$
\text { Idle Fraction Final }=\frac{\sum \text { Average Idle } \text { Time }_{i}}{\sum \text { Average Operating } \text { Time }_{i}}
$$

Where $\mathrm{i}=$ individual vehicle ID

This calculation was performed for idle fraction, workday idle, and extended idle by vehicle source type and day type. Idle fraction was also calculated within a vehicle source type (e.g. short-haul combination truck) by state of operation (e.g. California), and vocation (e.g. beverage delivery). In addition, the hourly idle fraction was calculated by vehicle type, day type, and hour ID using the same equations.

\subsubsection{Start Fraction}

Within the MOVES model, start fraction distributes engine starts temporally throughout the day. Current start estimates in MOVES are based on a limited data set of heavy-duty commercial vehicles, prompting the concern that the model may not adequately represent the wide range of heavy-duty vocations. Further, additional data allow for differentiation between vehicle types resulting in a more robust and higher resolved answer.

Starts are calculated using the engine rpm data channel and identifying instances when the engine rpm transitions from zero to greater than zero reporting a start instance each time the vehicle starts.

To calculate the start fraction for an individual vehicle and specific day type (weekday or weekend), the sum of the starts taking place within a specific hour ID, and day type, are divided by the sum of all starts occurring for that day type as shown with Equation 6. If the data logger was installed but did not record any activity, the start fraction is zero; however, if the data logger was not installed on a specific day type, those values are denoted as "nan" (not-a-number) and are removed from the results. The sum of the hourly fractions across all hours of the day is one.

$$
\text { Start Fraction } \text { Fr }_{h}=\frac{\sum \text { Hourly starts }}{\sum \text { Starts }}
$$

$$
\text { Where } h=\text { hour ID (1-24) }
$$


Like for idle fraction, two approaches were used to calculate the start fraction for a given day type and vehicle type. In the first approach, we averaged the start fractions calculated from Equation 6 across all vehicles within a vehicle type and day type. This approach weighted all vehicles the same, including vehicles that had very few starts.

For the second approach, starts within each day type and hour of the day are counted and then divided by the number of unique days of measurement to get a daily-average starts-per-hour for each individual vehicle. The average hourly starts from each individual vehicle are added together within each hour and divided by the average daily starts of each vehicle for each day type, and vehicle type (Equation 7). In this approach, the start fraction is influenced more by the vehicles which have the most starts per day. The start fraction using this approach is labeled as start_fraction_final in the data files.

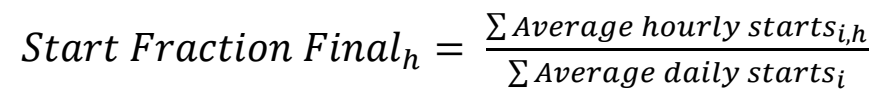

$$
\text { Where } i=\text { individual vehicle ID }
$$

$$
h=\text { hour ID (1-24) }
$$

This calculation was performed for each source type and day type. Start fraction was also calculated within a vehicle source type by state of operation, and vocation (See Appendix D for result files).

\subsubsection{Soak Fraction}

Emissions from vehicle starts are dependent on the vehicle's engine temperature prior to start. In MOVES, the starts are classified since last active operation or "soak" time. Vehicle soak is defined as the time between when an engine stops and the next time the engine starts. This is calculated by taking the time difference between when the engine speed transitions from greater than zero to zero for the stop and from zero to greater than zero for the start. This calculation is shown in Equation 8, and the results are binned according to the soak length bin defined in Table 3 , resulting in an output table containing soak length bin and timestamps.

$$
\text { Soak Time }=\text { Start Time }- \text { Stop Time }(\text { prior to the start })
$$

To calculate the soak fraction for an individual vehicle, the sum of the starts of a given soak time (also referred to as operating mode, o) within a specific hour ID and day type, are divided by the sum of all starts occurring for that hour ID and day type as shown with Equation 9. The sum of the eight soak fractions will equal one within the day type and hour of the day. If no starts occurred within that day type and hour ID, the sum of the eight soak length bin fractions would equal zero.

$$
\text { Soak } \text { Fraction }_{o}=\frac{\sum \text { Starts }_{o}}{\sum \text { Starts }}
$$

Where o = soak length bin, or operating mode ID (101-108) 
Table 3. MOVES Soak Time Operation Modes

\begin{tabular}{|ll|}
\hline MOVES Bin ID & Soak Length Bin Description \\
\hline 101 & Soak Time $<6$ minutes \\
\hline 102 & 6 minutes $\leq$ Soak Time $<30$ minutes \\
\hline 103 & 30 minutes $\leq$ Soak Time $<60$ minutes \\
\hline 104 & 60 minutes $\leq$ Soak Time $<90$ minutes \\
\hline 105 & 90 minutes $\leq$ Soak Time $<120$ minutes \\
\hline 106 & 120 minutes $\leq$ Soak Time $<360$ minutes \\
\hline 107 & 360 minutes $\leq$ Soak Time $<720$ minutes \\
\hline 108 & 720 minutes $\leq$ Soak Time \\
\hline
\end{tabular}

Like for the idle and start fractions, two approaches were used to calculate the soak fraction by vehicle source type. In the first approach, we averaged the soak fractions calculated from Equation 9 across all vehicles within a vehicle source type, day type, and hour of the day. This approach weighted all vehicles the same, including vehicles that had very little starts in certain hours of the day.

For the second approach, starts within each soak length bin, day type and hour of the day are counted and then divided by the number of unique days of measurement to get an average hourly starts by soak length bin and day type for each individual vehicle. The average hourly starts by soak length bin from each individual vehicle are added together and divided by the sum of all starts for each hour of the day, day type, and vehicle type (Equation 10). In this approach, the soak fraction is influenced more by the vehicles which have the most starts in that hour of the day. The soak fraction using this approach is labeled as soak_fraction_final in the data files.

$$
\begin{aligned}
& \text { Soak Fraction Final }{ }_{o}=\frac{\sum \text { Average hourly starts }}{\sum, o} \\
& \sum \text { Average hourly starts } \\
& \text { Where } i=\text { individual vehicle ID } \\
& o=\text { soak length bin, or operating mode ID }(101-108)
\end{aligned}
$$

This calculation was performed for each source type and day type, and hour of the day. Soak fraction was also calculated within a vehicle source type by state of operation, and vocation (See Appendix D for result files). 


\section{Results and Discussion}

This analysis used data from 415 conventional heavy-duty vehicles with over 120,000 hours of operation, providing a diverse data set encompassing 23 vehicle vocations in 36 states. The current resulting set includes idle, start, and soak fractions for the six vehicle types provided in Table 1 with a summary of vehicle type averaged results shown in Table 4.

Table 4. Summary Statistics²: Weekday Averages by Vehicle Type

\begin{tabular}{|lccccc|}
\hline Vehicle Type & Daily Operation & Starts per Day & Workday Idle & Extended Idle & Soak Length \\
\hline Transit Bus & $9.04 \mathrm{hrs}$ & 8.59 & $36.32 \%$ & $4.54 \%$ & $3.74 \mathrm{hrs}$ \\
\hline School Bus & $2.45 \mathrm{hrs}$ & 5.41 & $30.98 \%$ & $0.19 \%$ & $17.15 \mathrm{hrs}$ \\
\hline Refuse & $5.19 \mathrm{hrs}$ & 2.73 & $47.99 \%$ & $0.05 \%$ & $18.84 \mathrm{hrs}$ \\
\hline $\begin{array}{l}\text { Single-Unit } \\
\text { Short-Haul }\end{array}$ & $3.07 \mathrm{hrs}$ & 36.26 & $31.47 \%$ & $2.33 \%$ & $7.20 \mathrm{hrs}$ \\
\hline $\begin{array}{l}\text { Comb.-Unit } \\
\text { Short-Haul }\end{array}$ & $6.55 \mathrm{hrs}$ & 12.25 & $31.02 \%$ & $1.74 \%$ & $7.73 \mathrm{hrs}$ \\
\hline $\begin{array}{l}\text { Comb.-Unit } \\
\text { Long-Haul }\end{array}$ & $9.64 \mathrm{hrs}$ & 0.81 & $15.27 \%$ & $12.59 \%$ & $19.11 \mathrm{hrs}$ \\
\hline
\end{tabular}

On average, single and combination unit short-haul vehicles showed the largest number of starts per day, low extended idle fraction, and short soak lengths, whereas the combination long-haul vehicles showed the fewest starts per day, highest extended idle fraction, and long average soak length. Further, the average starts per day for combination long-haul is less than one due to the vehicle not operating some days, possible team trucking where drivers switch between shifts, and the limited amount of stops long-haul trucks make. The soak length for the combination trucks is the largest at over 19 hours. This is likely due to the vehicle not operating on some days, including weekend days.

Transit buses had the highest average daily operating time and lowest average soak length, indicating a high utilization compared to school buses and refuse trucks which had low average daily operation and long soak lengths. Finally, all vehicle types had workday idle fractions double that of combination-unit long-haul trucks as well as higher average starts per day, suggesting a more transient drive cycle from the local operations than that of the vehicles in the long-haul class. Appendix E provides further frequency distributions of starts per day and idle.

\subsection{Results by Vehicle Type}

The first analyses examined start, idle, and soak fractions of each vehicle type using the analysis approach described earlier. Figure 2 shows the vehicle type-specific start fraction multiplied by the soak bin fraction as a function of hour of the day for weekdays providing context as to the types of start emissions and the relative impact for each hour. Across all hours of the day, the

\footnotetext{
${ }^{2}$ Daily operation, starts per day, and soak length are calculated on a vehicle basis (each vehicle is weighted equally). Work-day idle and extended idle are calculated using the final approach outlined in Section 2.2.3 which is weighted to the average vehicle operation times.
} 
start fraction sums to 1, within each hour of the day, the soak bin fraction sums to 1 . By multiplying the two together, the graphs display the percentage of starts that occur at a given hour of the day and of a given soak length. For example, school buses have a $100 \%$ soak fraction of greater than 12 hours for hour six, suggesting the engine will be cold and there is a high potential for cold-start emissions during that hour, but the overall start fraction is small for hour 6, and most of the starts occur in the later morning and afternoon hours.

For the short- and long-haul vehicles, the largest portion of starts took place around midday, coinciding with operation during business hours with the short-haul vehicles having frequent starts with minimal soak time likely due to stopping for deliveries or implementation of idle shut-off programs such as the Clean Idle program in California. Conversely, combination longhaul trucks have a higher fraction of long soak periods which are indicative of long-haul trucking with limited stopping and most trips happening on highways. For refuse trucks, the majority of starts and largest portion of soak fractions longer than 12 hours occur between 5 a.m. and 8 a.m., corresponding to the start of the business day, suggesting this is the time period most drivers start their refuse collection routes.
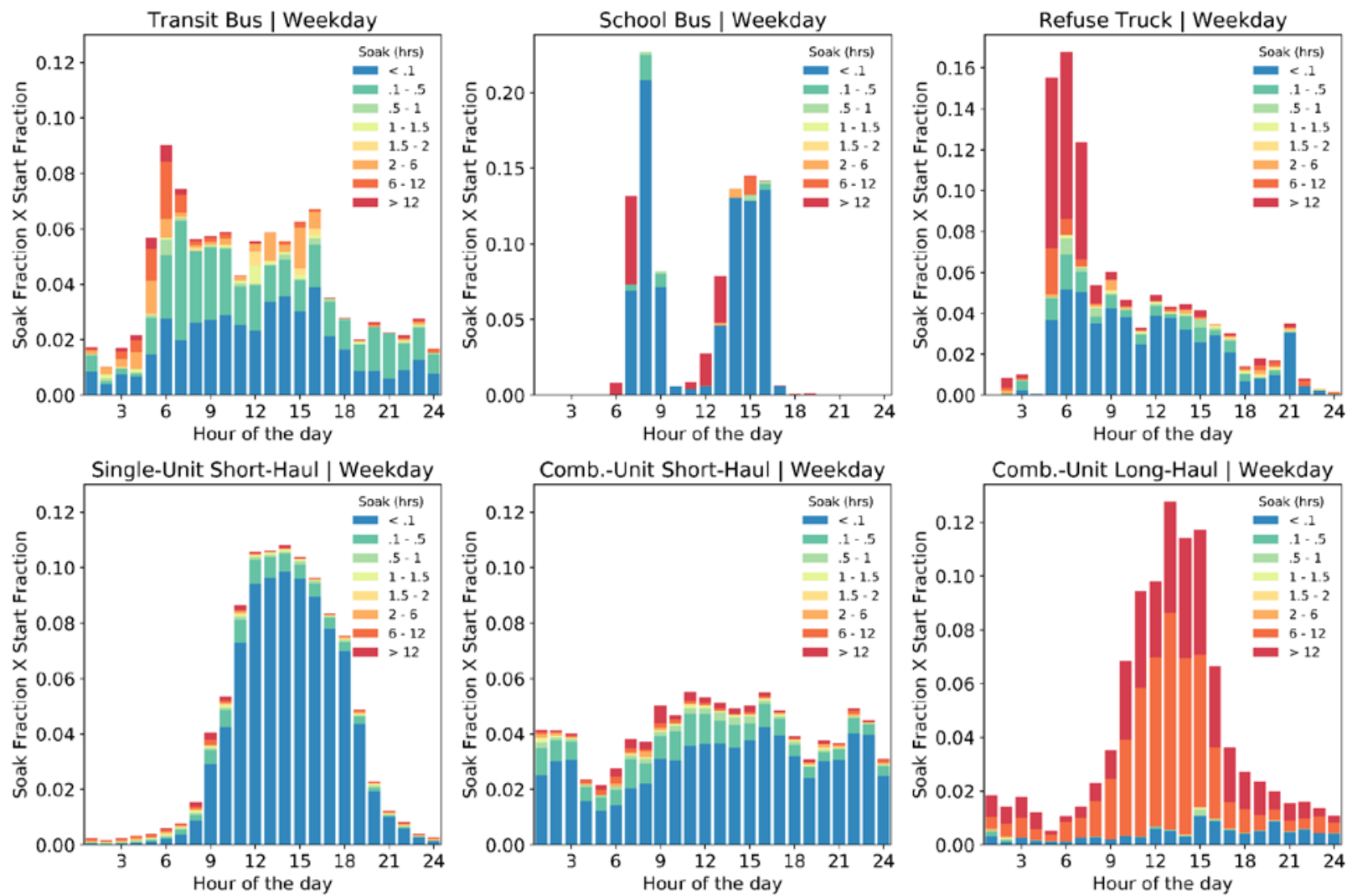

Figure 2. Soak fraction multiplied by start fraction vs. hour of the day for weekdays

Similarly, transit buses have the largest start fraction around 6 a.m.; however, the peak is less pronounced and at a similar level to the rest of the day in contrast to the refuse operation. Further, there are far shorter soak periods for the transit buses, indicating high utilization per vehicle. Finally, school buses show two very distinct peaks coinciding with the beginning and end of the school day as well as mid-day pickup, though due to the small sample size of vehicles 
in the school bus data set, values are likely to differ from the nation-wide average and do not cover summer operations. The remaining plots of soak bin fraction multiplied by start fraction by hour of the day are provided in Appendix F for each vehicle and day type.

Idle fraction is defined as the fraction of operation where the vehicle is traveling less than one $\mathrm{mph}$. The periods that last longer than one hour are considered extended idle periods. Figure 3 provides the workday and extended idle fractions by vehicle type and day type. Refuse trucks operating on weekdays have the largest idle fraction of all the vehicle and day types, whereas the school buses have no idle on weekends since none of the buses operated on those days. The longhaul class has the largest extended idle fraction of all vehicle types for both day types.

To gain a better understanding of idle fraction, Figure 4 examines weekday idle fraction multiplied by the fraction of operation as a function of hour and vehicle type ${ }^{3}$. Put another way, each bar in Figure 4 is the fraction of the total operation that the idle from that hour contributes. Short-haul vehicles have minimal extended idle throughout the day with workday idle being the main contributor and a peak around mid-day. Long-haul vehicles show a similar trend with peak idle just after mid-day, but there is a substantial contribution from extended idle that persists throughout all hours. This is likely a result of the engine idling while the driver is sleeping or after arriving at a depot. Both transit and school buses show morning and afternoon peaks that coincide with traffic for the transit bus and morning and afternoon pickup for school buses.

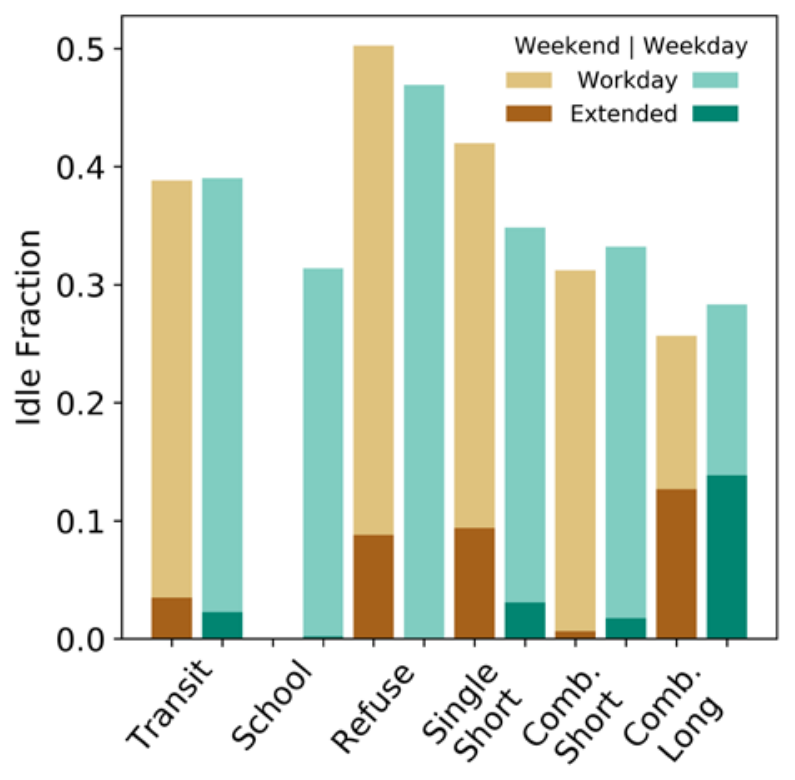

Figure 3. Extended and workday idle fractions vs. day type and vehicle type

\footnotetext{
${ }^{3}$ The operation fraction is the sum of operation within an hour divided by the sum of operation in all hours for a day and vehicle type. This estimates the percentage of vehicle operation that occurs at different hours of the day, and sums to 1 across all hours of the day. The idle fraction is the fraction of the total vehicle operation within each hour that is idling.
} 

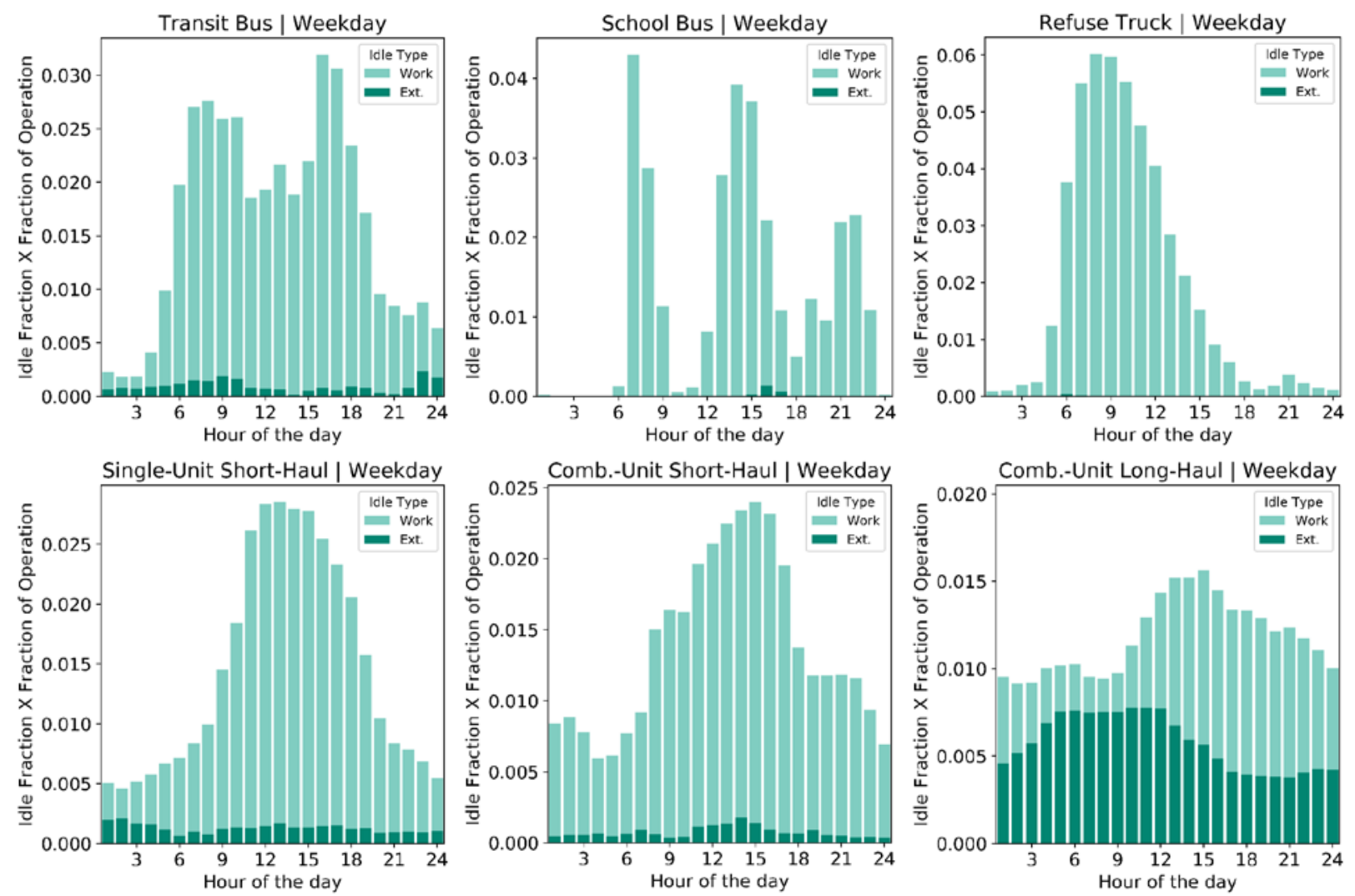

Figure 4. Extended and workday idle fractions vs. vehicle type for weekdays

Interestingly, the extended idle peaks for the transit buses in the morning, but peaks for the school buses in the afternoon. Finally, the plot for refuse trucks shows the majority of idle concentrated around business hours with the peak around 9 a.m. when refuse vehicles are making frequent stops at trash pickup locations. The plots for weekend day types are provided in Appendix G.

\subsection{Results by Vehicle Type and State}

Each vehicle type was further divided by state and re-analyzed to understand the differences in regional operation that may exist. One region-specific characteristic of interest is the effect of California's "clean idle" policy that requires vehicles not meeting idle emissions standards to shut down after idling a specified length of time depending on vocation. ${ }^{4}$ Table 5 provides the geographic locations of each vehicle type, indicating both the number of vehicles in each state and the fraction of the vehicle type population within that state. Location was determined by first using geographic information of the home base provided by the collecting agency. If information was not available, location was determined from the median latitude and longitude coordinates. In some instances, the data did not have geographic information of the home base, and the GPS was broken, in which case the location is designated as "Blank." The full result set for each state is provided in Appendix $\mathrm{H}$; however, a small subset of data is shown in Figure 5 to illustrate the regional differences in operation. California and Colorado single-unit short-haul vehicles have a

\footnotetext{
${ }^{4}$ In general, vehicles not meeting idle emissions standards are required to shut down after 5 minutes. Exceptions include passenger buses, which may idle for 10 minutes prior to passengers boarding, and school buses, which cannot idle longer than 30 seconds after arriving at or before departing from school.
} 
large portion of starts occurring with soak times less than six minutes, whereas vehicles from Washington have much longer soak times, which may be in part due to differing idle policies between the states. Looking at the soak fractions in Figure H.23 in Appendix H, we see that the single-unit short-haul vehicles from California do not have extended idling activity, whereas the vehicles from Colorado and Washington have small fractions of extended idle, thus confirming operational differences. It is unclear if vehicles from California show lower idle fractions and higher start counts due to the idle policy, or if other factors such as vocation and drive cycle contribute to these differences in operation.
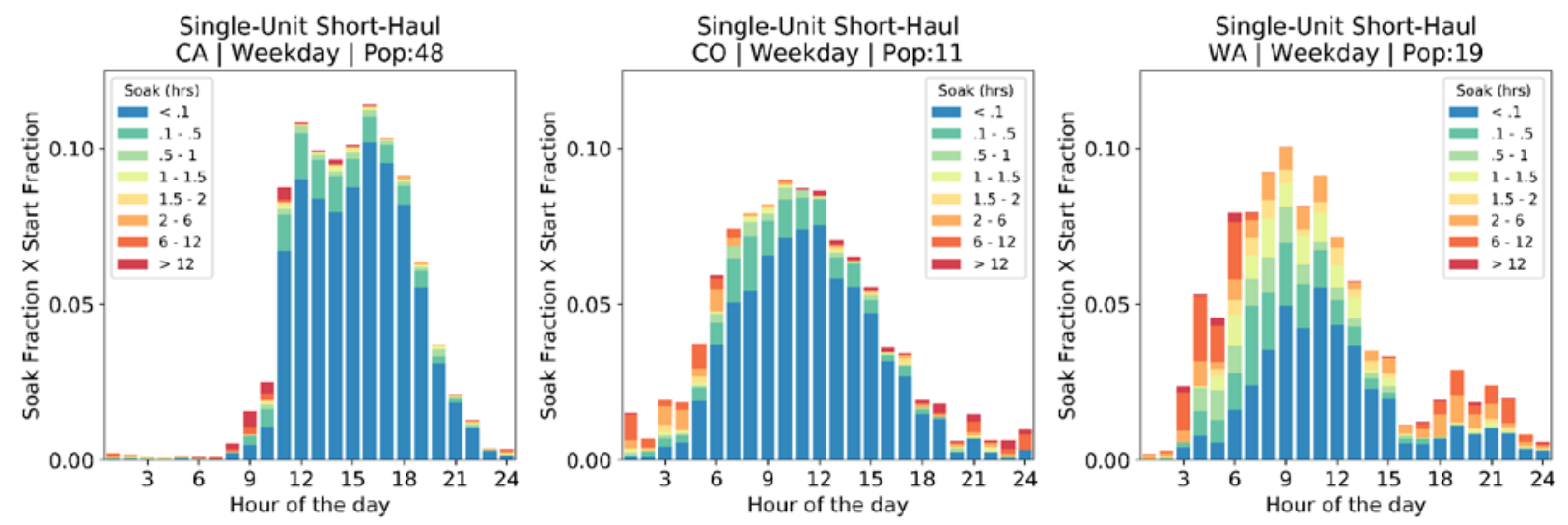

Figure 5. Start fraction times normalized soak fraction vs. hour for short-haul classes 
Table 5. Geographic Distribution of Data by Vehicle Type

\begin{tabular}{|c|c|c|c|c|c|c|c|c|}
\hline \multicolumn{3}{|c|}{ Transit Buses } & \multicolumn{3}{|c|}{ Comb.-Unit Short-Haul } & \multicolumn{3}{|c|}{ Comb.-Unit Long-Haul } \\
\hline State & Count & $\%$ & State & Count & $\%$ & State & Count & $\%$ \\
\hline $\mathrm{CO}$ & 4 & $25.0 \%$ & CA & 65 & $61.9 \%$ & $A L$ & 1 & $0.8 \%$ \\
\hline $\mathrm{MN}$ & 9 & $56.3 \%$ & $\mathrm{CO}$ & 13 & $12.4 \%$ & AR & 5 & $3.8 \%$ \\
\hline $\mathrm{TN}$ & 3 & $18.8 \%$ & FL & 1 & $1.0 \%$ & $A Z$ & 5 & $3.8 \%$ \\
\hline Blank & 0 & $0.0 \%$ & MD & 4 & $3.8 \%$ & $\mathrm{CA}$ & 14 & $10.7 \%$ \\
\hline Total & 16 & $100.0 \%$ & $\mathrm{MI}$ & 6 & $5.7 \%$ & $\mathrm{CO}$ & 5 & $3.8 \%$ \\
\hline \multirow{2}{*}{\multicolumn{3}{|c|}{ School Buses }} & TN & 3 & $2.9 \%$ & ID & 1 & $0.8 \%$ \\
\hline & & & TX & 6 & $5.7 \%$ & IL & 7 & $5.3 \%$ \\
\hline State & Count & $\%$ & WI & 1 & $1.0 \%$ & IN & 4 & $3.1 \%$ \\
\hline CA & 4 & $57.1 \%$ & Blank & 6 & $5.7 \%$ & KS & 4 & $3.1 \%$ \\
\hline Blank & 3 & $42.9 \%$ & Total & 105 & $100.0 \%$ & KY & 1 & $0.8 \%$ \\
\hline Total & 7 & $100.0 \%$ & & & & MD & 1 & $0.8 \%$ \\
\hline \multirow{2}{*}{\multicolumn{3}{|c|}{ Refuse Trucks }} & & & & MN & 2 & $1.5 \%$ \\
\hline & & & \multicolumn{3}{|c|}{ Single-Unit Short-Haul } & $\mathrm{MO}$ & 3 & $2.3 \%$ \\
\hline State & Count & $\%$ & State & Count & $\%$ & MS & 1 & $0.8 \%$ \\
\hline $\mathrm{CO}$ & 7 & $18.9 \%$ & CA & 48 & $40.3 \%$ & MT & 1 & $0.8 \%$ \\
\hline FL & 11 & $29.7 \%$ & $\mathrm{CO}$ & 11 & $9.2 \%$ & NC & 1 & $0.8 \%$ \\
\hline IN & 13 & $35.1 \%$ & IN & 9 & $7.6 \%$ & ND & 4 & $3.1 \%$ \\
\hline $\mathrm{OH}$ & 4 & $10.8 \%$ & $\mathrm{MN}$ & 11 & $9.2 \%$ & NE & 1 & $0.8 \%$ \\
\hline Blank & 2 & $5.4 \%$ & TN & 3 & $2.5 \%$ & NM & 3 & $2.3 \%$ \\
\hline \multirow[t]{15}{*}{ Total } & 37 & $100.0 \%$ & $\mathrm{TX}$ & 9 & $7.6 \%$ & NV & 6 & $4.6 \%$ \\
\hline & & & UT & 1 & $0.8 \%$ & NY & 1 & $0.8 \%$ \\
\hline & & & WA & 19 & $16.0 \%$ & $\mathrm{OH}$ & 5 & $3.8 \%$ \\
\hline & & & Blank & 8 & $6.7 \%$ & OK & 13 & $9.9 \%$ \\
\hline & & & Total & 119 & $100.0 \%$ & OR & 3 & $2.3 \%$ \\
\hline & & & & & & PA & 3 & $2.3 \%$ \\
\hline & & & & & & SC & 1 & $0.8 \%$ \\
\hline & & & & & & $S D$ & 2 & $1.5 \%$ \\
\hline & & & & & & $\mathrm{TN}$ & 13 & $9.9 \%$ \\
\hline & & & & & & $\mathrm{TX}$ & 3 & $2.3 \%$ \\
\hline & & & & & & UT & 7 & $5.3 \%$ \\
\hline & & & & & & WA & 6 & $4.6 \%$ \\
\hline & & & & & & $W Y$ & 4 & $3.1 \%$ \\
\hline & & & & & & Blank & 0 & $0.0 \%$ \\
\hline & & & & & & Total & 131 & $100.0 \%$ \\
\hline
\end{tabular}




\subsection{Results by Vehicle Type, State, and Vocation}

Single-unit and combination-unit short-haul classes contain multiple vocations compared to the others and would be more sensitive to vocational differences. Table 6 provides a breakdown of vocations for both short-haul vehicle types along with the average daily distance for each vocation to highlight the operational differences among the vocations.

Table 6. Average Daily Distance by Vocation for Short-Haul Vehicle Types

Single-Unit Short Haul

\begin{tabular}{|lcc|}
\hline Vocation & Vehicles & Avg. Miles \\
\hline Concrete & 3 & 79.79 \\
\hline Delivery & 1 & 294.41 \\
\hline Dump Truck & 3 & 70.53 \\
\hline Food Delivery & 30 & 49.88 \\
\hline Linen Delivery & 17 & 65.65 \\
\hline Parcel Delivery & 39 & 52.47 \\
\hline Propane Tank & 1 & 6.72 \\
\hline Shredder & 1 & 9.15 \\
\hline Snow Plow & 11 & 27.12 \\
\hline Towing & 4 & 33.79 \\
\hline Warehouse Delivery & 9 & 79.21 \\
\hline
\end{tabular}

Combination-Unit Short Haul

\begin{tabular}{|lcc|}
\hline Vocation & Vehicles & Avg. Miles \\
\hline Beverage Delivery & 10 & 95.71 \\
\hline Drayage & 28 & 119.18 \\
\hline Dry Van & 3 & 99.13 \\
\hline Dump Truck & 4 & 143.77 \\
\hline Food Delivery & 13 & 161.52 \\
\hline Local Delivery & 7 & 101.31 \\
\hline Parcel Delivery & 5 & 573.83 \\
\hline Regional Haul & 7 & 118.34 \\
\hline Transfer Truck & 28 & 174.27 \\
\hline
\end{tabular}

The five combination-unit parcel delivery trucks travel nearly 500 miles a day on average compared to the beverage delivery trucks, which travel less than 100 miles per day. The start and soak fractions for a subset of those vehicles, shown in Figure 6, illustrate the stark operational differences as well. The beverage delivery vehicles have their peak starts during normal business hours whereas these parcel delivery vehicles have a relatively flat distribution of starts around the clock. This is a result of the parcel delivery vehicles operating multiple shifts with local delivery during the day and inter-depot delivery at night.
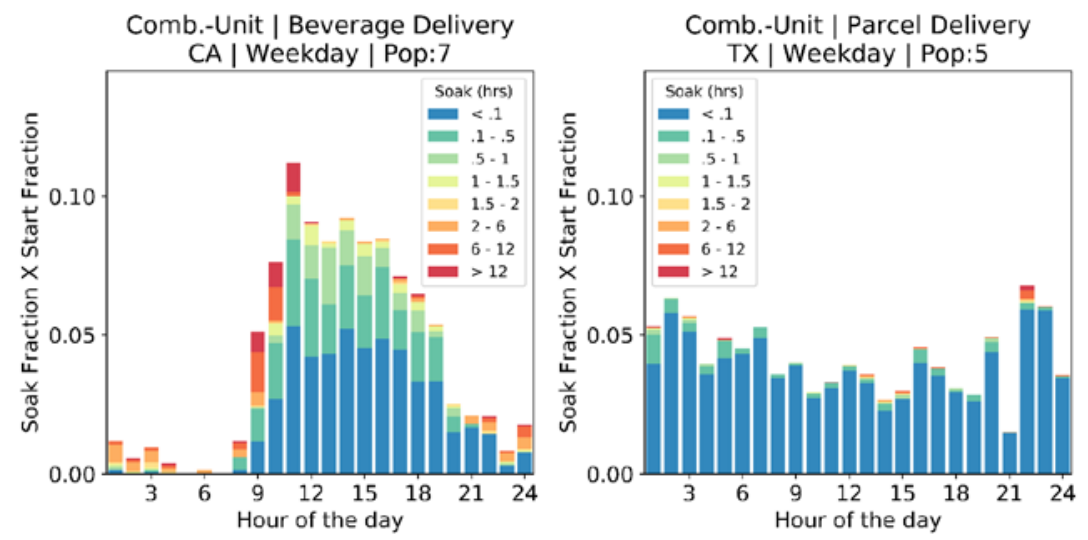

Figure 6. Vocational differences between beverage and parcel delivery combination trucks

Narrowing the scope to a single state and short-haul vocation type, Figure 7 shows that distinct differences exist between various vocations of delivery vehicle. The linen delivery vehicles show the highest fraction of starts around 2 p.m. and longer soak times towards the beginning of the 
day whereas the parcel delivery vehicles have the bulk starts in the afternoon, and the warehouse delivery vehicles show starts in nearly all hours of the day. The shapes of these start distributions, soak fractions associated with each start, and the populations of vehicles within the analysis may impact emissions timing throughout the day. The remaining start and soak distributions are shown in Appendix I.
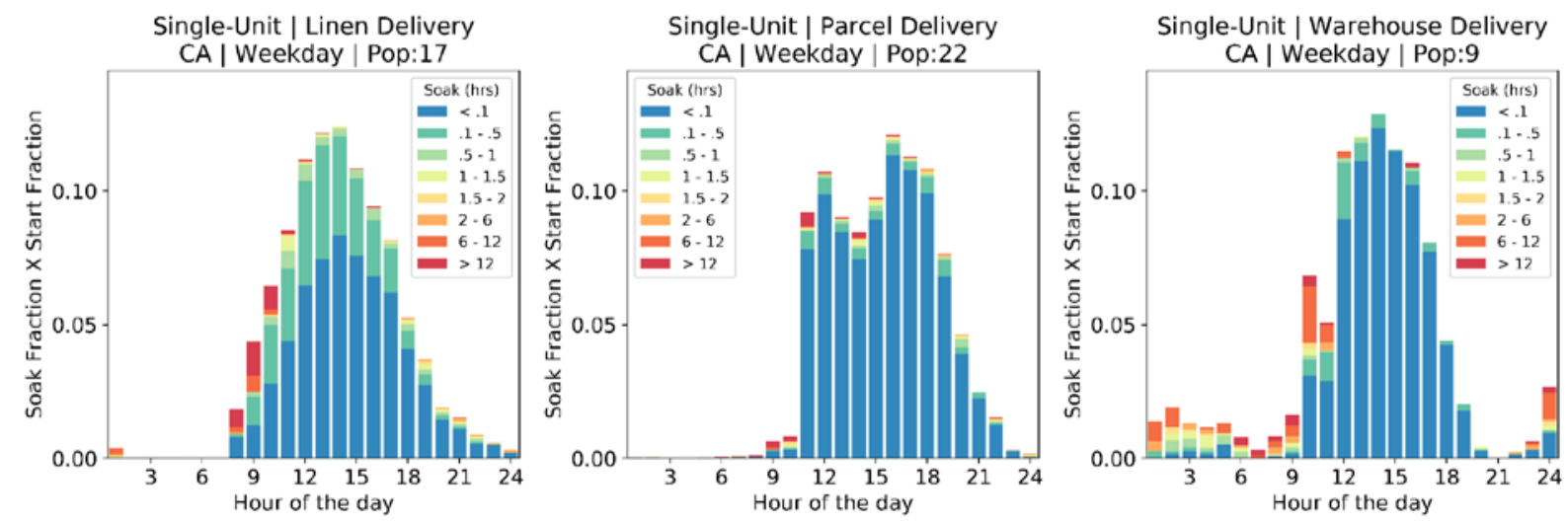

Figure 7. Start and soak distributions for single-unit California delivery vehicles 


\section{Conclusion}

This analysis used data from NREL's Fleet DNA database to provide heavy-duty fleet information as inputs informing the EPA's MOVES model. Six of the nine MOVES heavy-duty vehicle types were available in Fleet DNA, providing data from 415 conventional, dieselpowered vehicles with over 120,000 hours of operation. Start fraction, soak fraction, and idle fraction by hour of the day were derived for each vehicle type, state, and vocation. Results were provided in the form of .CSV files that can be easily translated to MOVES inputs. A list of the files is included in Appendix D. This midterm report detailed these results, providing graphical analysis and context for the start, soak, and idle distributions. Future analysis will include vehicle speed distributions broken down by the road type classifications provided in Table A.1. 


\section{Appendix A: MOVES Definitions}

Table A.1. MOVES Road Types

\begin{tabular}{|lll|}
\hline MOVES Road ID & $\begin{array}{l}\text { Road Type } \\
\text { Off Network }\end{array}$ & $\begin{array}{l}\text { FHWA Functional Types } \\
\text { Off Network }\end{array}$ \\
\hline 1 & Rural Restricted Access & Rural Interstate \\
\hline 3 & Rural Unrestricted Access & $\begin{array}{l}\text { Rural Principal Arterial, Minor Arterial, Major } \\
\text { Collector, Minor Collector, \& Local }\end{array}$ \\
\hline 4 & Urban Restricted Access & Urban Interstate \& Urban Freeway/Expressway \\
\hline 5 & Urban Unrestricted Access & $\begin{array}{l}\text { Urban Principal Arterial, Minor Arterial, Collector, \& } \\
\text { Local }\end{array}$ \\
\hline
\end{tabular}

Table A.2. Heavy-Duty Vehicle MOVES Vehicle Types

\begin{tabular}{|ll|}
\hline MOVES Vehicle ID & Vehicle Type \\
\hline 41 & Intercity Buses (non-school, non-transit) \\
\hline 42 & Transit Buses \\
\hline 43 & School Buses \\
\hline 51 & Refuse Trucks \\
\hline 52 & Single Unit Short-Haul Trucks \\
\hline 53 & Single Unit Long-Haul Trucks \\
\hline 54 & Motor Homes \\
\hline 61 & Combination Short-Haul Trucks \\
\hline 62 & Combination Long-Haul Trucks \\
\hline
\end{tabular}

Table A.3. MOVES Day Types

\begin{tabular}{|ll|}
\hline MOVES Day ID & Day Type \\
\hline 2 & Weekend Days: Saturday and Sunday \\
\hline 5 & Weekdays: Monday through Friday \\
\hline
\end{tabular}


Table A.4. MOVES Hour Types

\begin{tabular}{|c|c|}
\hline MOVES Hour ID & Hour \\
\hline 1 & Hour beginning at $12: 00$ midnight \\
\hline 2 & Hour beginning at 1:00 a.m. \\
\hline 3 & Hour beginning at 2:00 a.m. \\
\hline 4 & Hour beginning at 3:00 a.m. \\
\hline 5 & Hour beginning at 4:00 a.m. \\
\hline 6 & Hour beginning at 5:00 a.m. \\
\hline 7 & Hour beginning at 6:00 a.m. \\
\hline 8 & Hour beginning at 7:00 a.m. \\
\hline 9 & Hour beginning at 8:00 a.m. \\
\hline 10 & Hour beginning at 9:00 a.m. \\
\hline 11 & Hour beginning at 10:00 a.m. \\
\hline 12 & Hour beginning at 11:00 a.m. \\
\hline 13 & Hour beginning at $12: 00$ Noon \\
\hline 14 & Hour beginning at 1:00 p.m. \\
\hline 15 & Hour beginning at 2:00 p.m. \\
\hline 16 & Hour beginning at 3:00 p.m. \\
\hline 17 & Hour beginning at 4:00 p.m. \\
\hline 18 & Hour beginning at 5:00 p.m. \\
\hline 19 & Hour beginning at 6:00 p.m. \\
\hline 20 & Hour beginning at 7:00 p.m. \\
\hline 21 & Hour beginning at 8:00 p.m. \\
\hline 22 & Hour beginning at 9:00 p.m. \\
\hline 23 & Hour beginning at $10: 00$ p.m. \\
\hline 24 & Hour beginning at 11:00 p.m. \\
\hline
\end{tabular}


Table A.5. MOVES Soak Time Operation Modes

\begin{tabular}{|ll|}
\hline MOVES Bin ID & Soak Length Bin Description \\
\hline 101 & Soak Time $<6$ minutes \\
\hline 102 & 6 minutes $\leq$ Soak Time $<30$ minutes \\
\hline 103 & 30 minutes $\leq$ Soak Time $<60$ minutes \\
\hline 104 & 60 minutes $\leq$ Soak Time $<90$ minutes \\
\hline 105 & 90 minutes $\leq$ Soak Time $<120$ minutes \\
\hline 106 & 120 minutes $\leq$ Soak Time $<360$ minutes \\
\hline 107 & 360 minutes $\leq$ Soak Time $<720$ minutes \\
\hline 108 & 720 minutes $\leq$ Soak Time \\
\hline
\end{tabular}




\section{Appendix B: Short-Haul Vocation Definitions}

Note: All vehicles are representative examples and are not the actual vehicles tested.

\section{Combination Short Haul}

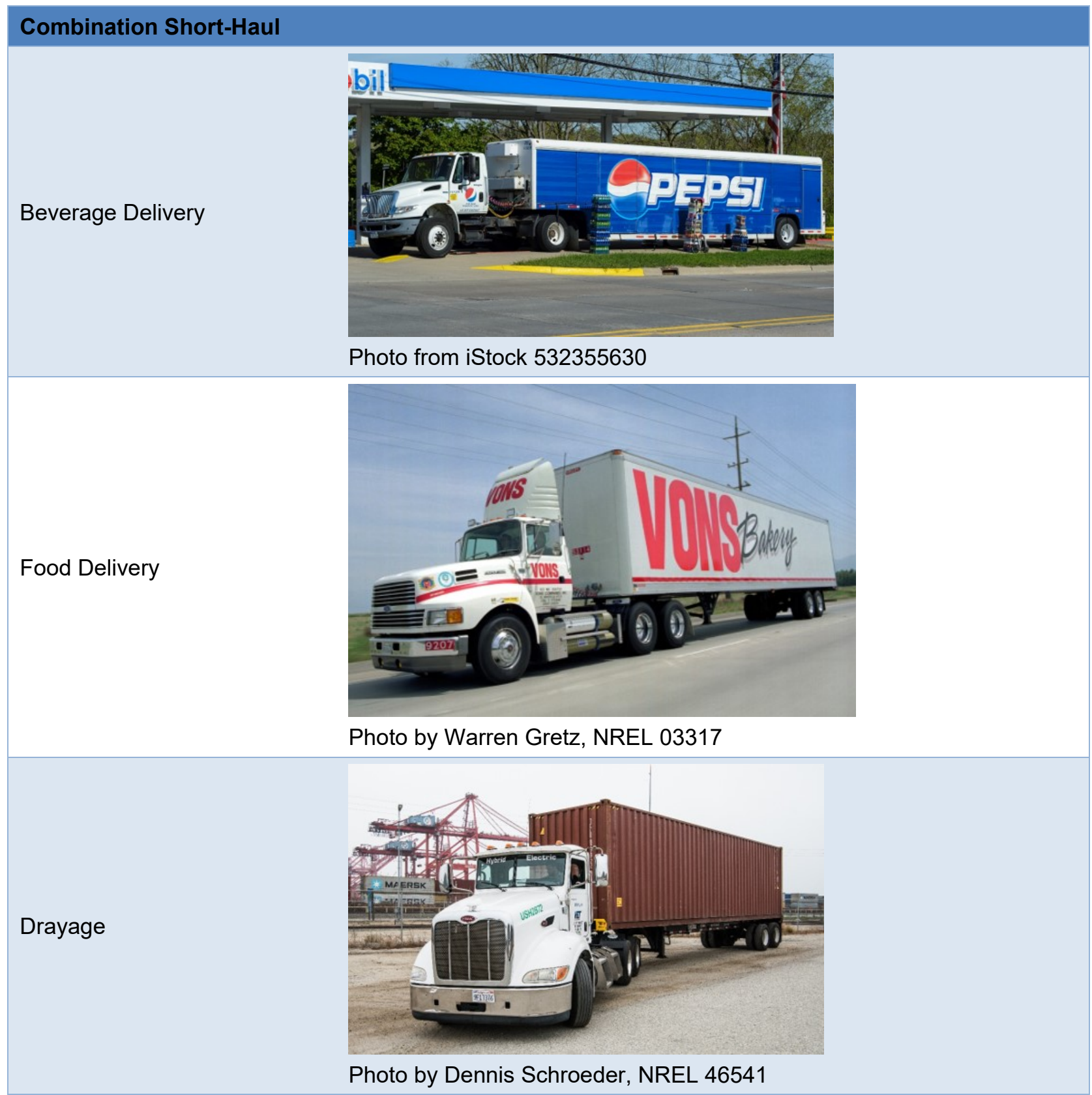




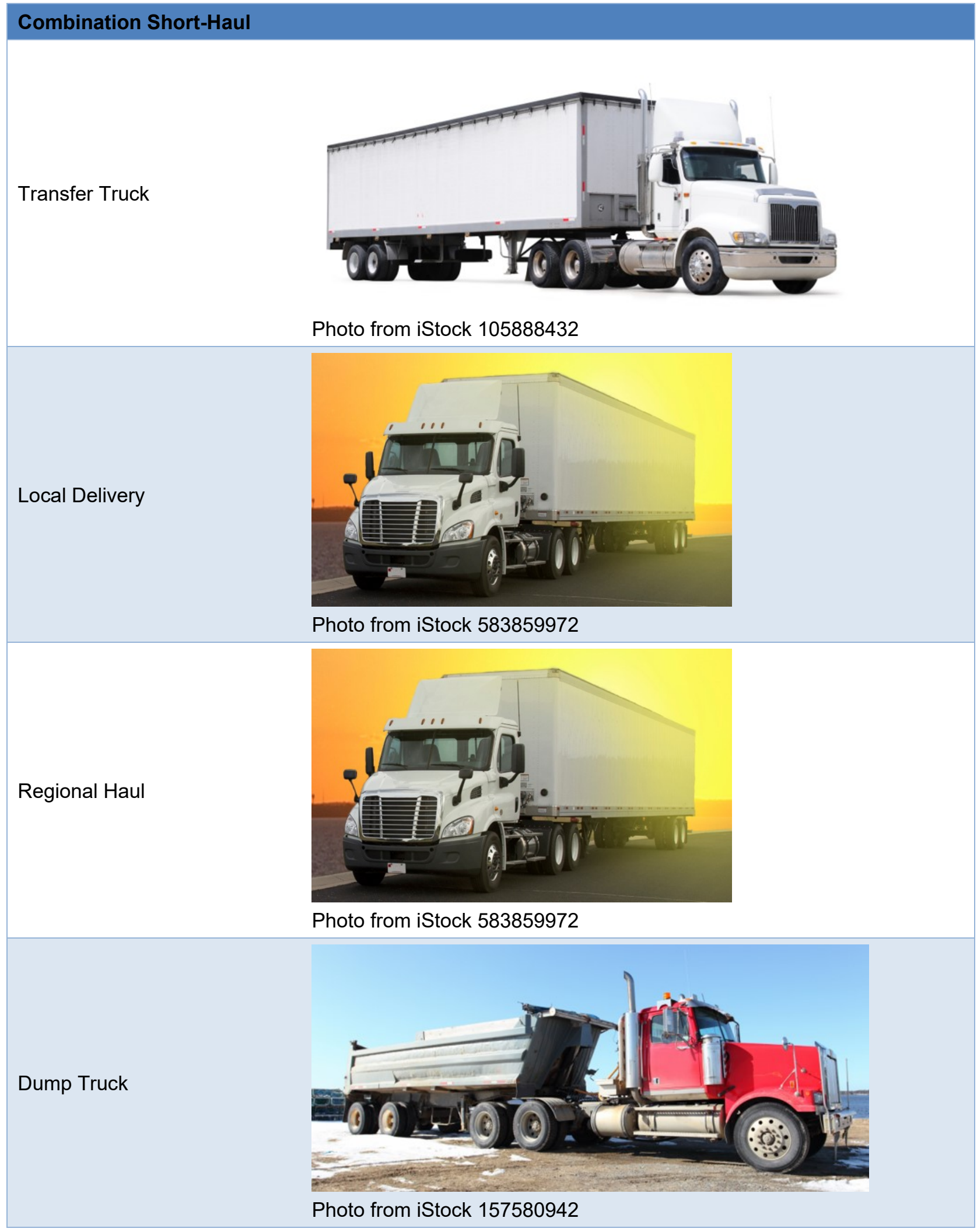




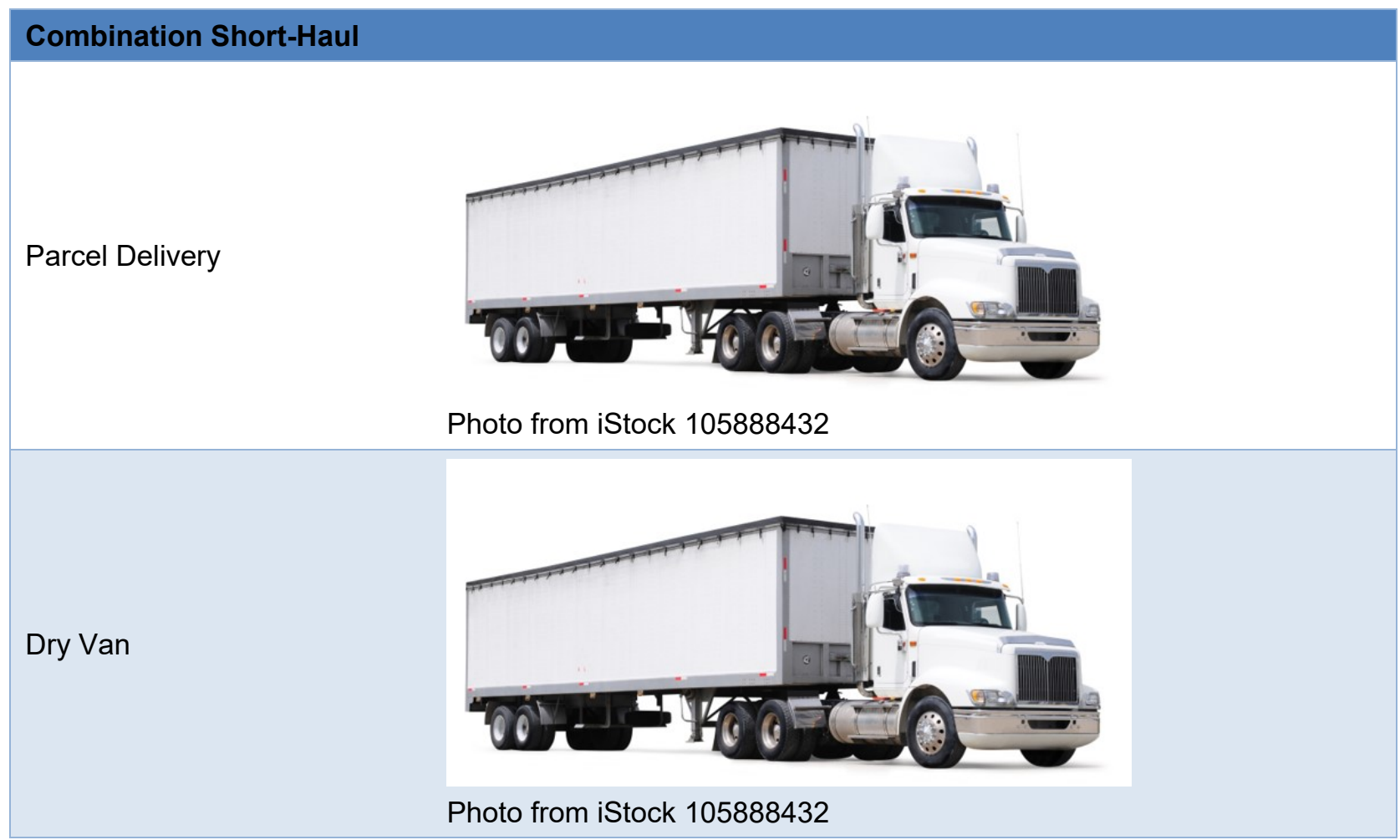

\section{Single-Unit Short Haul}

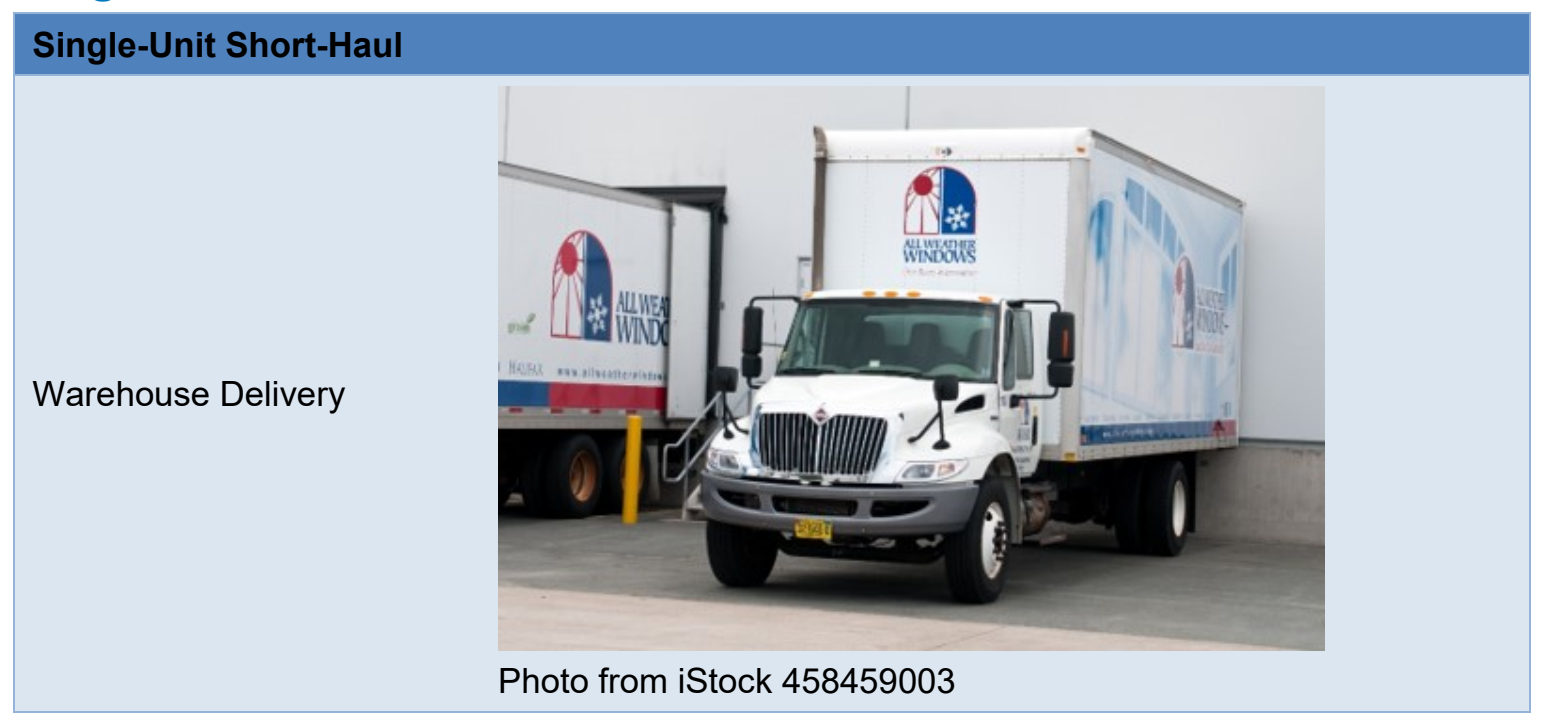




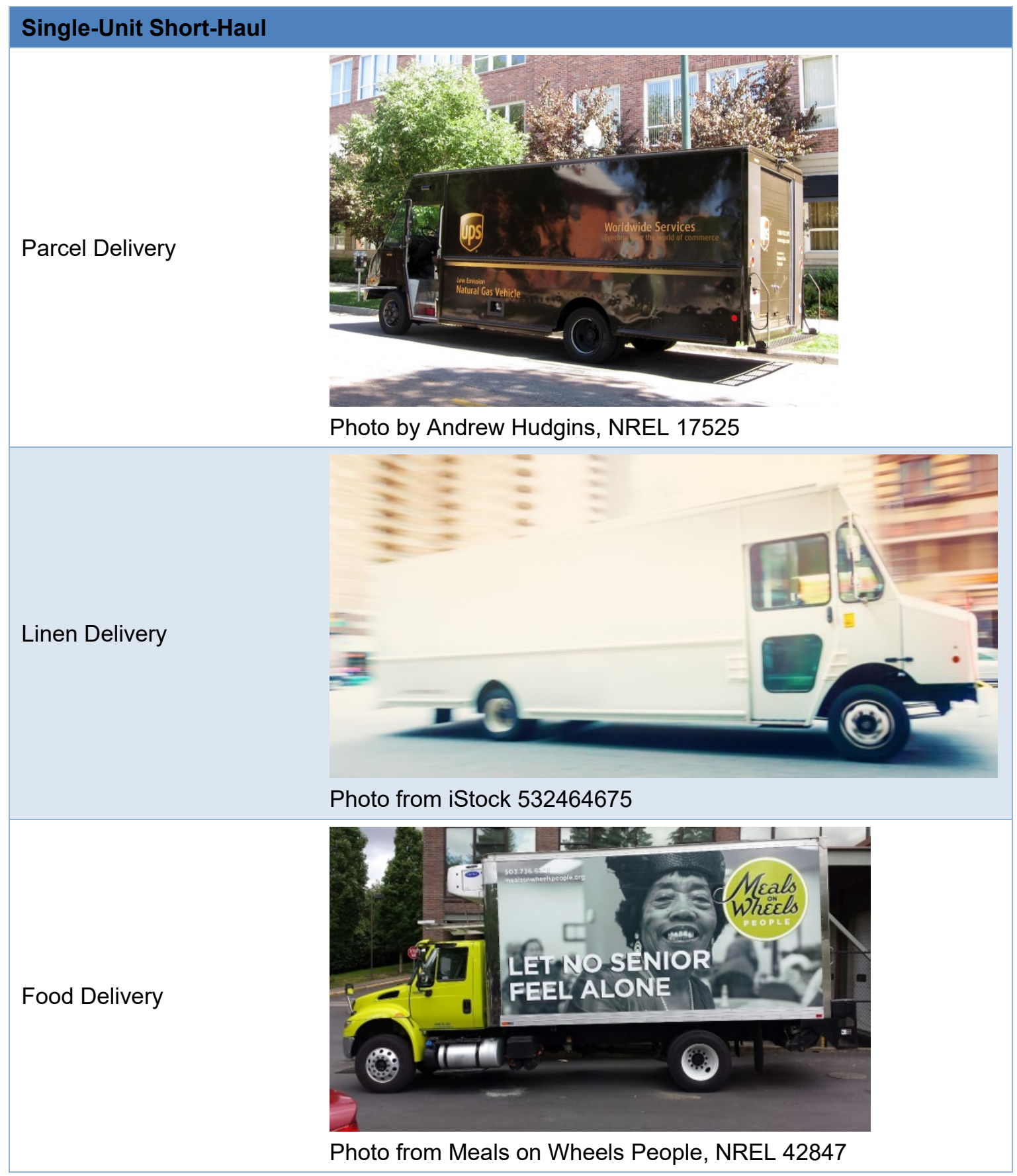




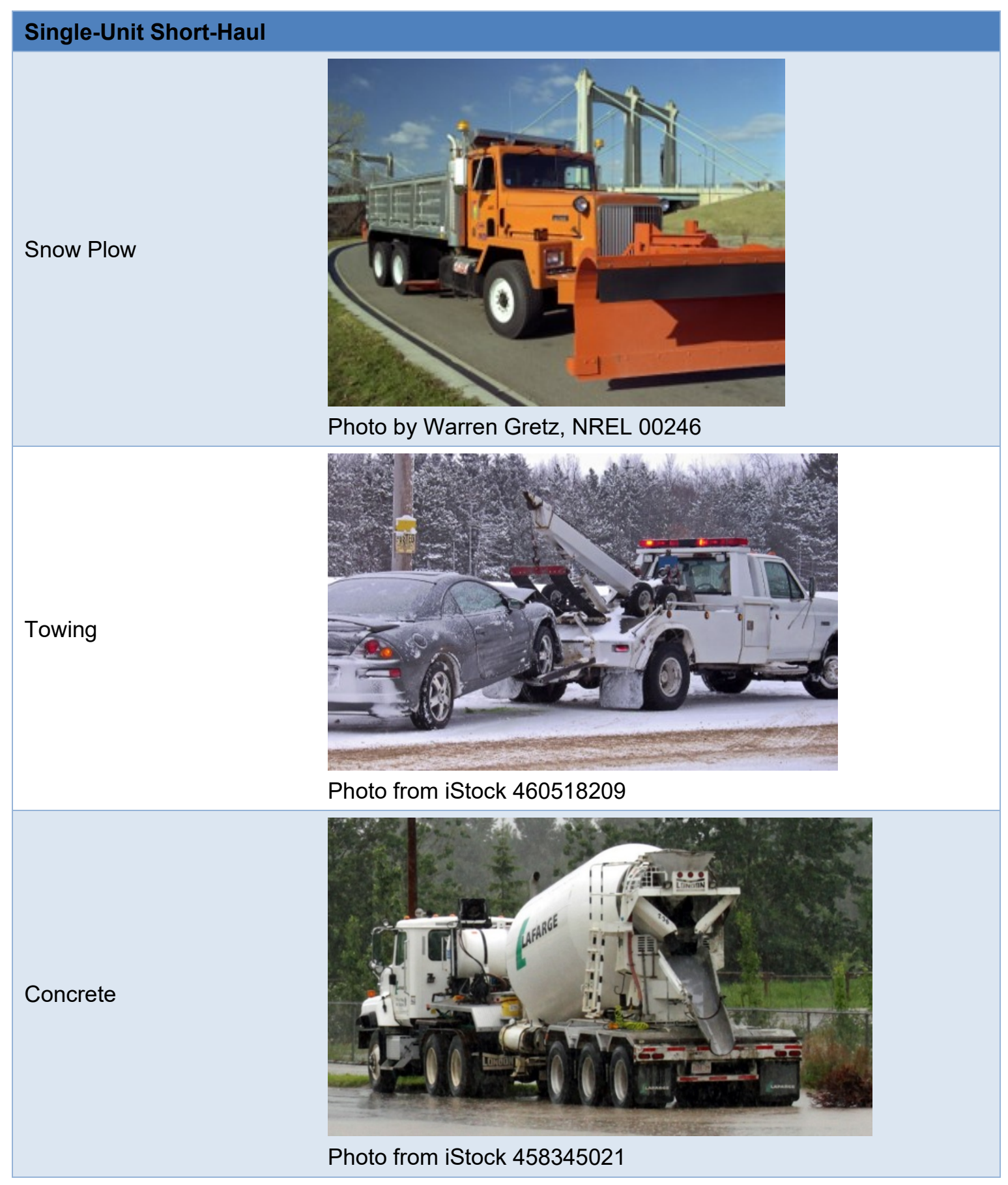




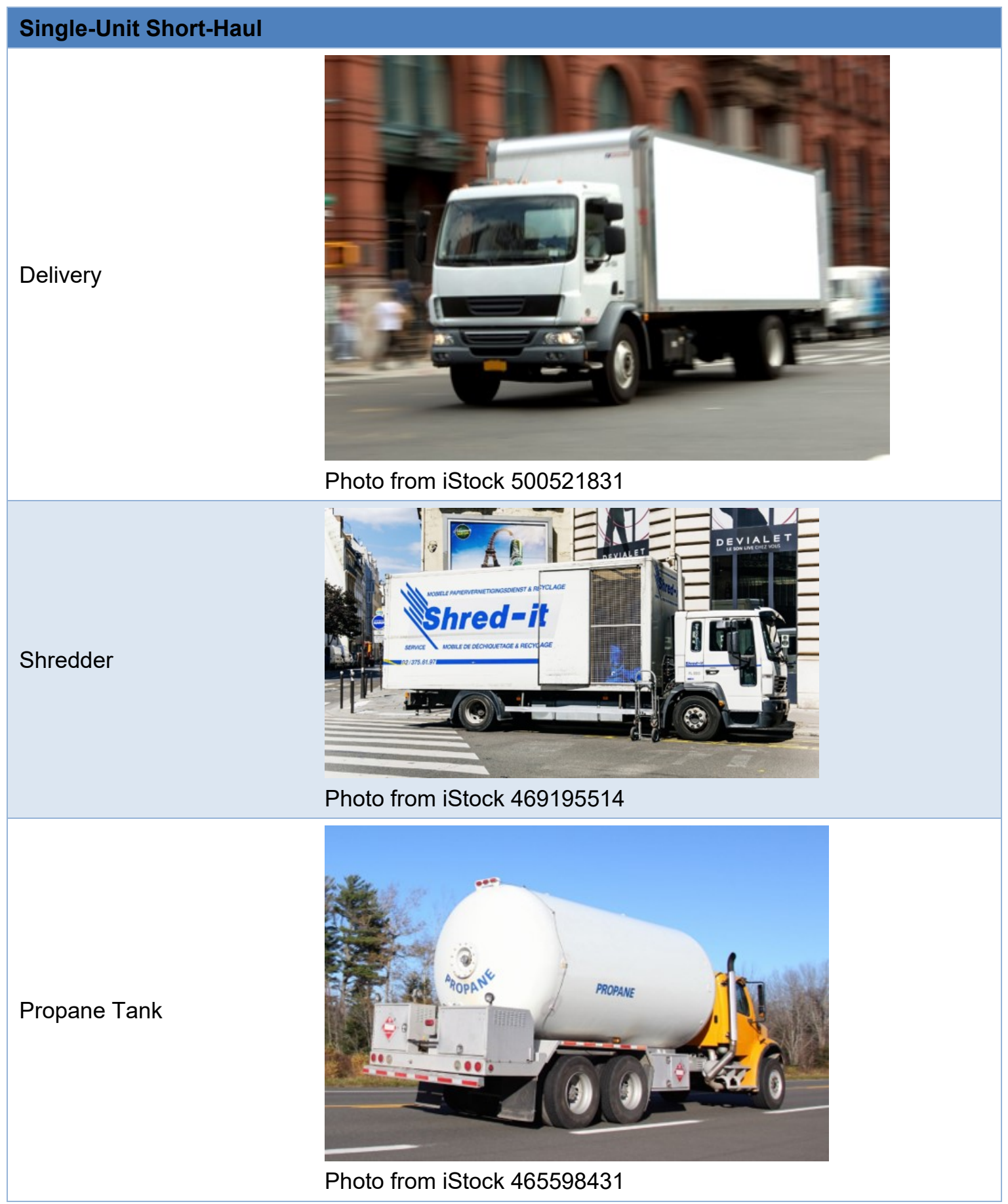




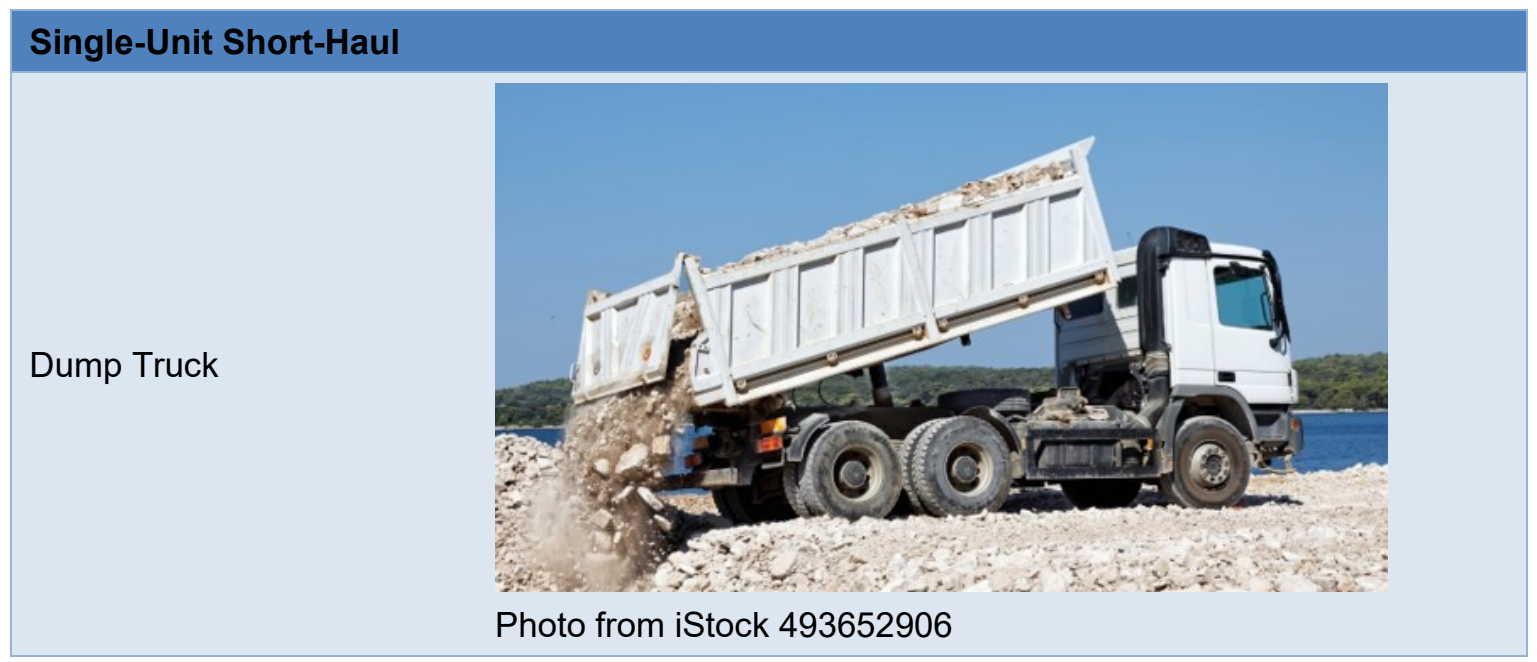




\section{Appendix C: Gap Length Analysis}

As mentioned in Section 2.2.2, when a gap occurred in the timestamp of the recorded vehicle activity without a corresponding engine start (the engine speed did not fall to zero), we implemented an algorithm that would add an engine start (by setting the engine speed to zero) depending on the length of the gap in the timestamp (referred to as the gap length).

To aid in the selection of a gap length at which to add starts, we examined the sensitivity of the vehicle soak bin distribution and start fraction by adding starts at different gap lengths. Plots of vehicle soak bin distribution multiplied by start fraction for gap lengths varying between 2 seconds and 30 hours are provided below. The result for the gap length of 30 hours was nearly identical to the result if the gap-filling algorithm was not used and should be considered as the baseline result. Figure C.1 shows the distributions for combination-unit long-haul trucks that have very few starts per day. Figure C.2 provides the distributions for single-unit short-haul trucks that have many starts per day. The gap length algorithm had the most noticeable effect on the long-haul trucks due to the high weighting placed on each start as a result of having very few starts per day. In other words, the starts added by the gap-filling algorithm, constituted a large percentage of the overall starts from these vehicles. As shown in Figure C.2, the choice of the gap length has less of an impact on the start fraction and soak fractions for single-unit short-haul vehicles because these vehicles had more recorded starts in the baseline case (gap length $>30$ hours), and were less influenced by imputing starts for gap lengths that occurred $<30$ hours.

Because combination long-haul trucks were the most affected by the gap length, we used the gap length chosen from combination long-haul (6 hours) on all other heavy-duty source types.

Six hours was selected as the gap length because it seemed to provide the most realistic results for long-haul trucks for two reasons: 1) the six-hour gaps minimized the percentage of starts occurring in the early morning (midnight to $6 \mathrm{am}$ ). The shorter gap lengths appeared to put an unrealistically high amount of starts in the early morning hours (midnight to 6 a.m.). 2) Gap length of greater than six hours appears to concentrate the start activity in the early afternoon hours, while also removing an excessive amount of the starts that occurred during the late morning hours (9 am -12 noon).

We believe that adding starts to gap lengths longer than 6 hours is a reasonable approach to correctly classify engine-off events that likely occurred during these long timestamp gaps, while avoiding overestimating the number of starts during short gap lengths where it is likely no engine-off events occurred, such as during a GPS timestamp updates.

A quantitative study of correctness could not be performed due to most data having some form of timestamp gap, so engineering judgement was used to identify a gap length that was likely to avoid categorizing legitimate time gaps as starts while still providing additional data points over the baseline result. It is possible that vehicle soaks less than six hours were missed; however, without knowing the unique caveats of each data set, it is challenging to quantify this. 

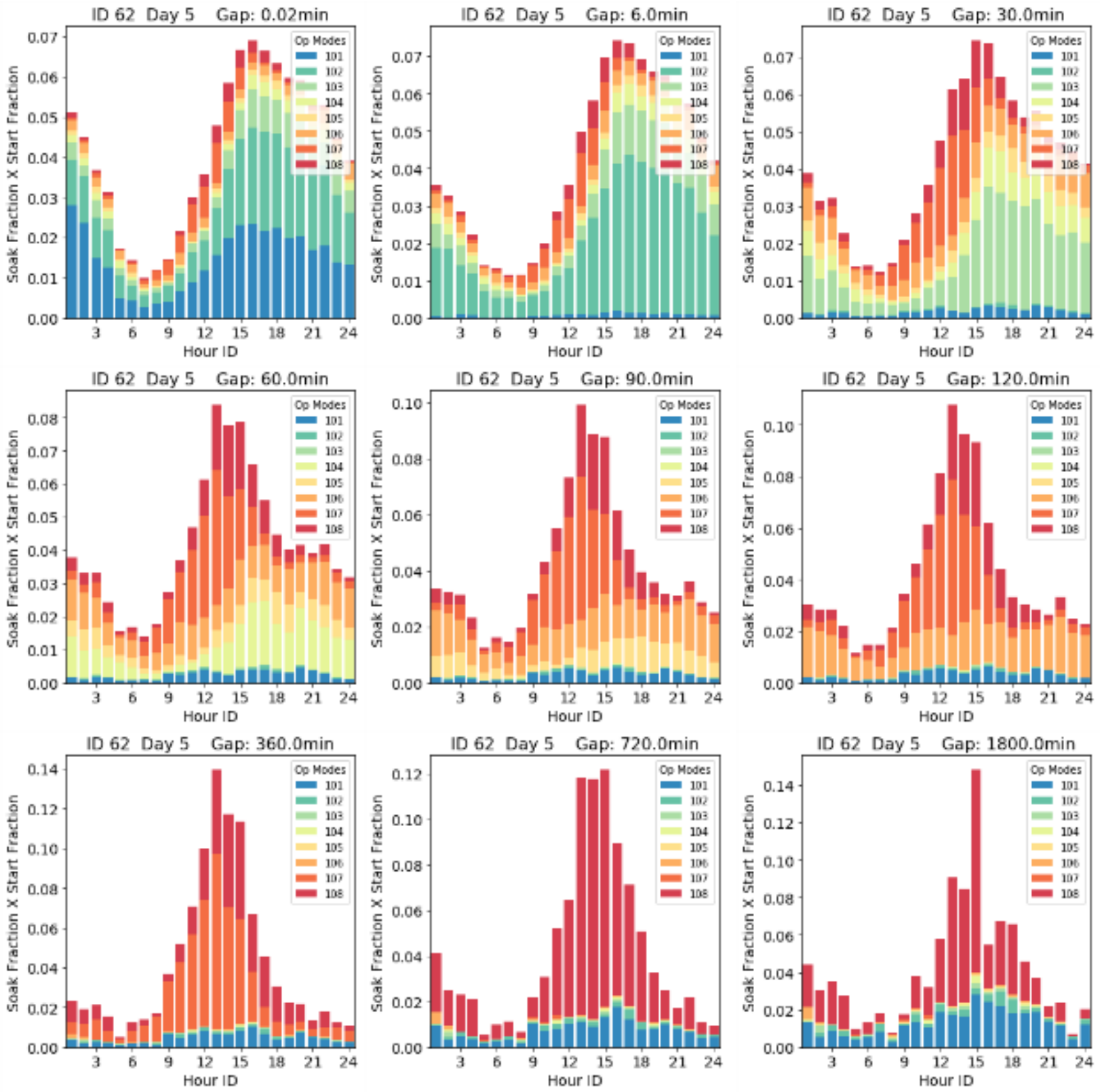

Figure C.1. Start fraction weighted soak distribution by gap length: Comb.-Unit Long-Haul 

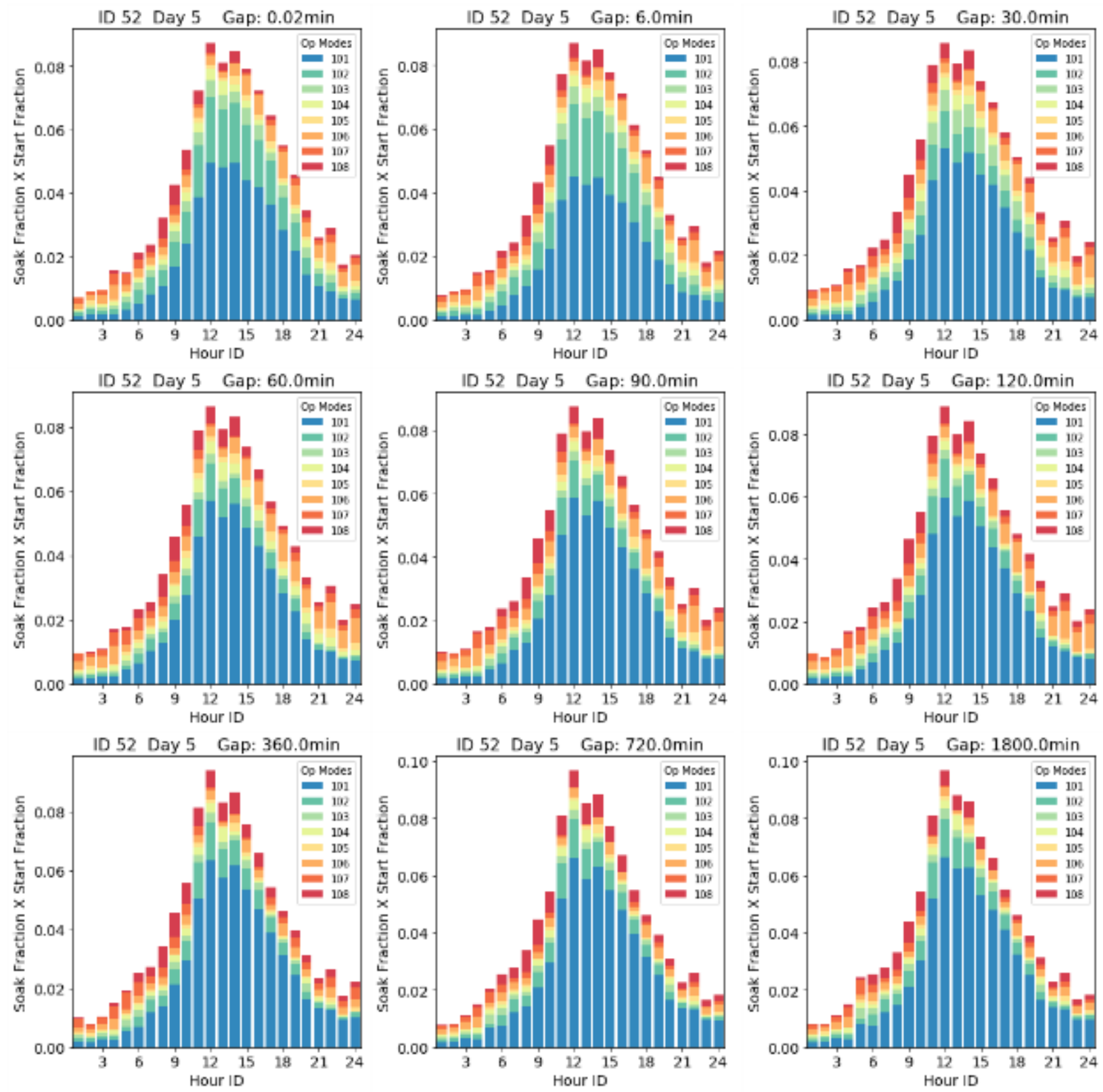

Figure C.2. Start fraction weighted soak distribution by gap length: Single-Unit Short-Haul 


\section{Appendix D: Results File List}

\section{Full Results Set File List}

\section{starts_final_102518.csv}

- This file provides the start fraction for each source type (vehicle type) and day type along with statistics of the data. The full list of parameters included in the file is as follows:

\begin{tabular}{|l|l|}
\hline Column Name & Description \\
\hline source_type & Source Type \\
\hline day & Day Type (weekday=5, weekend = 2) \\
\hline hour & Hour of the day (1-24) \\
\hline start_fraction_final & $\begin{array}{l}\text { Sum of the daily average starts for an individual hour, day and vehicle type divided by } \\
\text { the sum of the daily average starts for an individual day and vehicle type }\end{array}$ \\
\hline sum_starts & Sum of the daily average starts per hour, day and vehicle type \\
\hline population & Population of vehicles for that source type and day type \\
\hline avg_starts_per_day & Average starts per day \\
\hline
\end{tabular}

\section{soak_final_102518.csv}

- This file provides the soak fraction for each source type (vehicle type), day type, hour of the day and operation mode. The full list of parameters included in the file is as follows:

\begin{tabular}{|l|l|}
\hline Column Name & Description \\
\hline source_type & Source Type \\
\hline day & Day Type (weekday=5, weekend = 2) \\
\hline hour & Hour of the day (1-24) \\
\hline op_mode & Operation mode of the soaks (101-108) \\
\hline soak_fraction_final & $\begin{array}{l}\text { Sum of the daily average soaks for an individual op-mode, hour, day and vehicle type } \\
\text { divided by the sum of daily average starts for an individual hour, day and vehicle type }\end{array}$ \\
\hline sum_soaks & Sum of the daily average soaks per op-mode, hour, day and vehicle type \\
\hline
\end{tabular}

\section{idle_final_102518.csv}

- This file provides the idle fraction for each source type (vehicle type) and day type along with statistics of the data. The full list of parameters included in the file is as follows:

\begin{tabular}{|l|l|}
\hline Column Name & Description \\
\hline source_type & Source Type \\
\hline day & Day Type (weekday=5, weekend = 2) \\
\hline idle_fraction_final & $\begin{array}{l}\text { Sum of the daily average idle hours for all vehicles divided by sum } \\
\text { of the daily average operating hours for all vehicles in the day and } \\
\text { vehicle type }\end{array}$ \\
\hline workday_idle_fraction_final & $\begin{array}{l}\text { Sum of the daily average workday idle hours for all vehicles } \\
\text { divided by sum of the daily average operating hours for all vehicles } \\
\text { in the day and vehicle type }\end{array}$ \\
\hline
\end{tabular}




\begin{tabular}{|l|l|} 
extended_idle_fraction_final & $\begin{array}{l}\text { Sum of the daily average extended idle hours for all vehicles } \\
\text { divided by sum of the daily average operating hours for all vehicles } \\
\text { in the day and vehicle type }\end{array}$ \\
\hline sum_operation_hours & $\begin{array}{l}\text { Sum of the daily average operating hours for all vehicles in the day } \\
\text { and vehicle type }\end{array}$ \\
\hline sum_idle_hours & $\begin{array}{l}\text { Sum of the daily average idle hours for all vehicles in the day and } \\
\text { vehicle type }\end{array}$ \\
\hline sum_workday_idle_hours & $\begin{array}{l}\text { Sum of the daily average workday idle hours for all vehicles in the } \\
\text { day and vehicle type }\end{array}$ \\
\hline sum_extended_idle_hours & $\begin{array}{l}\text { Sum of the daily average extended idle hours for all vehicles in the } \\
\text { day and vehicle type }\end{array}$ \\
\hline sum_miles_driven & $\begin{array}{l}\text { Sum of the average daily miles driven for that day type and vehicle } \\
\text { type }\end{array}$ \\
\hline extended_idle_hours/miles_driven & $\begin{array}{l}\text { Sum of the daily average extended idle hours for all vehicles } \\
\text { divided by sum of the daily average miles traveled for all vehicles } \\
\text { in the day and vehicle type }\end{array}$ \\
\hline population & Population of vehicles for that source type and day type \\
\hline
\end{tabular}

\section{hourly_idle_final_102518.csv}

- This file provides the total, workday and extended idle fraction for each source type (vehicle type), day type and hour of the day along with statistics of the data. The full list of parameters included in the file is as follows:

\begin{tabular}{|l|l|}
\hline Column Name & Description \\
\hline source_type & Source Type \\
\hline day & Day Type (weekday=5, weekend = 2) \\
\hline hour & Hour of the day (1-24) \\
\hline idle_fraction_final & $\begin{array}{l}\text { Sum of the daily average idle hours for all vehicles divided by sum of the } \\
\text { daily average operating hours for all vehicles in hour, day and vehicle type }\end{array}$ \\
\hline workday_idle_fraction_final & $\begin{array}{l}\text { Sum of the daily average workday idle hours for all vehicles divided by } \\
\text { and vehicle type }\end{array}$ \\
\hline extended_idle_fraction_final & $\begin{array}{l}\text { Sum of the daily average operating hours for all vehicles in the hour, day } \\
\text { and vehicle type }\end{array}$ \\
\hline sum_operating_hours & $\begin{array}{l}\text { Sum of the daily average operating hours for all vehicles in the hour, day } \\
\text { and vehicle type }\end{array}$ \\
\hline sum_idle_hours & $\begin{array}{l}\text { Sum of the daily average idle hours for all vehicles in the hour, day and } \\
\text { vehicle type }\end{array}$ \\
\hline sum_workday_idle_hours & $\begin{array}{l}\text { Sum of the daily average workday idle hours for all vehicles in the hour, } \\
\text { day and vehicle type }\end{array}$ \\
\hline sum_extended_idle_hours & $\begin{array}{l}\text { Sum of the daily average extended idle hours for all vehicles in the hour, } \\
\text { day and vehicle type }\end{array}$ \\
\hline
\end{tabular}




\section{Results by State File List}

\section{starts_by_state_final_102518.csv}

- This file provides the start fraction for each state, source type (vehicle type) and day type along with statistics of the data. The full list of parameters included in the file is as follows:

\begin{tabular}{|l|l|}
\hline Column Name & Description \\
\hline state & State of origin or most frequent operation \\
\hline source_type & Source Type \\
\hline day & Day Type (weekday=5, weekend = 2) \\
\hline hour & Hour of the day (1-24) \\
\hline start_fraction_final & $\begin{array}{l}\text { Sum of the daily average starts for an individual hour, vehicle type, day type and state } \\
\text { and state }\end{array}$ \\
\hline sum_starts & Sum of the daily average starts per hour, vehicle type, day type and state \\
\hline population & Population of vehicles for that vehicle type, day type and state \\
\hline avg_starts_per_day & Average starts per day \\
\hline
\end{tabular}

\section{soak_by_state_final_102518.csv}

- This file provides the soak fraction for each state, source type (vehicle type), day type, hour of the day and operation mode. The full list of parameters included in the file is as follows:

\begin{tabular}{|l|l|}
\hline Column Name & Description \\
\hline state & State of origin or most frequent operation \\
\hline source_type & Source Type \\
\hline day & Day Type (weekday=5, weekend $=2)$ \\
\hline hour & Hour of the day $(1-24)$ \\
\hline op_mode & Operation mode of the soaks $(101-108)$ \\
\hline soak_fraction_final & $\begin{array}{l}\text { Sum of the daily average soaks for an individual op-mode, hour, vehicle type, day type } \\
\text { type day type and state }\end{array}$ \\
\hline sum_soaks & Sum of the daily average soaks per op-mode, hour, vehicle type, day type and state \\
\hline
\end{tabular}

\section{idle_by_state_final_102518.csv}

- This file provides the idle fraction for each state, source type (vehicle type) and day type along with statistics of the data. The full list of parameters included in the file is as follows:

\begin{tabular}{|l|l|}
\hline Column Name & Description \\
\hline state & State of origin or most frequent operation \\
\hline source_type & Source Type \\
\hline day & Day Type (weekday=5, weekend = 2) \\
\hline idle_fraction_final & $\begin{array}{l}\text { Sum of the daily average idle hours for all vehicles divided by } \\
\text { sum of the daily average operating hours for all vehicles in the } \\
\text { vehicle type, day type and state }\end{array}$ \\
\hline
\end{tabular}




\begin{tabular}{|l|l|} 
workday_idle_fraction_final & $\begin{array}{l}\text { Sum of the daily average workday idle hours for all vehicles } \\
\text { divided by sum of the daily average operating hours for all } \\
\text { vehicles in the vehicle type, day type and state }\end{array}$ \\
\hline extended_idle_fraction_final & $\begin{array}{l}\text { Sum of the daily average extended idle hours for all vehicles } \\
\text { divided by sum of the daily average operating hours for all } \\
\text { vehicles in the vehicle type, day type and state }\end{array}$ \\
\hline sum_operation_hours & $\begin{array}{l}\text { Sum of the daily average operating hours for all vehicles in the } \\
\text { vehicle type, day type and state }\end{array}$ \\
\hline sum_idle_hours & $\begin{array}{l}\text { Sum of the daily average idle hours for all vehicles in the vehicle } \\
\text { type, day type and state }\end{array}$ \\
\hline sum_workday_idle_hours & $\begin{array}{l}\text { Sum of the daily average workday idle hours for all vehicles in } \\
\text { the vehicle type, day type and state }\end{array}$ \\
\hline sum_extended_idle_hours & $\begin{array}{l}\text { Sum of the daily average extended idle hours for all vehicles in } \\
\text { the vehicle type, day type and state }\end{array}$ \\
\hline sum_miles_driven & $\begin{array}{l}\text { Sum of the average daily miles driven for that vehicle type, day } \\
\text { type and state }\end{array}$ \\
\hline & $\begin{array}{l}\text { Sum of the daily average extended idle hours for all vehicles } \\
\text { divided by sum of the daily average miles traveled for all vehicles } \\
\text { in the vehicle type, day type and state }\end{array}$ \\
\hline extended_idle_hours/miles_driven & Population of vehicles for that vehicle type, day type and state \\
\hline population
\end{tabular}

\section{Results by Vocation File List}

\section{starts_by_voc_final_102518.csv}

- This file provides the start fraction for each vocation, source type (vehicle type) and day type along with statistics of the data. The full list of parameters included in the file is as follows:

\begin{tabular}{|c|c|}
\hline Column Name & Description \\
\hline vocation & Vocation of the vehicle \\
\hline source_type & Source Type \\
\hline day & Day Type (weekday=5, weekend = 2) \\
\hline hour & Hour of the day (1-24) \\
\hline start_fraction_final & $\begin{array}{l}\text { Sum of the daily average starts for an individual hour, vehicle type, day type and } \\
\text { vocation divided by the sum of the daily average starts for an individual vehicle type, } \\
\text { day type and vocation }\end{array}$ \\
\hline sum_starts & Sum of the daily average starts per hour, vehicle type, day type and vocation \\
\hline population & Population of vehicles for that vehicle type, vocation, day type \\
\hline avg_starts_per_day & Average starts per day \\
\hline
\end{tabular}

\section{soak_by_voc_final_102518.csv}

- This file provides the soak fraction for each vocation, source type (vehicle type), day type, hour of the day and operation mode. The full list of parameters included in the file is as follows: 


\begin{tabular}{|l|l|}
\hline Column Name & Description \\
\hline vocation & Vocation of the vehicle \\
\hline source_type & Source Type \\
\hline day & Day Type (weekday=5, weekend = 2) \\
\hline hour & Hour of the day (1-24) \\
\hline op_mode & Operation mode of the soaks (101-108) \\
\hline soak_fraction_final & $\begin{array}{l}\text { Sum of the daily average soaks for an individual op-mode, hour, vehicle type, day type } \\
\text { and vocation divided by the sum of the daily average starts for individual hour, vehicle } \\
\text { type, day type and vocation }\end{array}$ \\
\hline sum_soaks & $\begin{array}{l}\text { Sum of the daily average soaks per op-mode, hour, vehicle type, day type and } \\
\text { vocation }\end{array}$ \\
\hline
\end{tabular}

\section{idle_by_voc_final_102518.csv}

- This file provides the idle fraction for each vocation, source type (vehicle type) and day type along with statistics of the data. The full list of parameters included in the file is as follows:

\begin{tabular}{|l|l|}
\hline Column Name & Description \\
\hline vocation & Vocation of the vehicle \\
\hline source_type & Source Type \\
\hline day & Day Type (weekday=5, weekend = 2) \\
\hline idle_fraction_final & $\begin{array}{l}\text { Sum of the daily average idle hours for all vehicles divided by } \\
\text { sum of the daily average operating hours for all vehicles in the } \\
\text { vehicle type, day type and vocation }\end{array}$ \\
\hline workday_idle_fraction_final & $\begin{array}{l}\text { Sum of the daily average workday idle hours for all vehicles } \\
\text { divided by sum of the daily average operating hours for all } \\
\text { vehicles in the vehicle type, day type and vocation }\end{array}$ \\
\hline extended_idle_fraction_final & $\begin{array}{l}\text { Sum of the daily average extended idle hours for all vehicles } \\
\text { divided by sum of the daily average operating hours for all } \\
\text { vehicles in the vehicle type, day type and vocation }\end{array}$ \\
\hline sum_operation_hours & $\begin{array}{l}\text { Sum of the daily average operating hours for all vehicles in the } \\
\text { vehicle type, day type and vocation }\end{array}$ \\
\hline sum_idle_hours & $\begin{array}{l}\text { Sum of the daily average idle hours for all vehicles in the vehicle } \\
\text { type, day type and vocation }\end{array}$ \\
\hline sum_workday_idle_hours & $\begin{array}{l}\text { Sum of the daily average workday idle hours for all vehicles in } \\
\text { the vehicle type, day type and vocation }\end{array}$ \\
\hline sum_extended_idle_hours & $\begin{array}{l}\text { Sum of the daily average extended idle hours for all vehicles in } \\
\text { the vehicle type, day type and vocation }\end{array}$ \\
\hline sum_miles_driven & $\begin{array}{l}\text { Sum of the average daily miles driven for that vehicle type, day } \\
\text { type and vocation }\end{array}$ \\
\hline extended_idle_hours/miles_driven & $\begin{array}{l}\text { Sum of the daily average extended idle hours for all vehicles } \\
\text { divided by sum of the daily average miles traveled for all vehicles } \\
\text { in the vehicle type, day type and vocation }\end{array}$ \\
\hline
\end{tabular}




\section{Results by State and Vocation File List}

\section{starts_by_state_and_voc_final_102518.csv}

- This file provides the start fraction for each state, vocation, source type (vehicle type) and day type along with statistics of the data. The full list of parameters included in the file is as follows:

\begin{tabular}{|l|l|}
\hline Column Name & Description \\
\hline state & State of origin or most frequent operation \\
\hline vocation & Vocation of the vehicle \\
\hline source_type & Source Type \\
\hline day & Day Type (weekday=5, weekend = 2) \\
\hline hour & Hour of the day (1-24) \\
\hline start_fraction_final & $\begin{array}{l}\text { Sum of the daily average starts for an individual hour, day type, vehicle type, state } \\
\text { vehicle type, state and vocation }\end{array}$ \\
\hline sum_starts & Sum of the daily average starts per hour, day type, vehicle type, state and vocation \\
\hline population & Population of vehicles for that vehicle type, vocation, state, and day type \\
\hline avg_starts_per_day & Average starts per day \\
\hline
\end{tabular}

\section{soak_by_state_and_voc_final_102518.csv}

- This file provides the soak fraction for each state, vocation, source type (vehicle type), day type, hour of the day and operation mode. The full list of parameters included in the file is as follows:

\begin{tabular}{|l|l|}
\hline Column Name & Description \\
\hline state & State of origin or most frequent operation \\
\hline vocation & Vocation of the vehicle \\
\hline source_type & Source Type \\
\hline day & Day Type (weekday=5, weekend = 2) \\
\hline hour & Hour of the day (1-24) \\
\hline op_mode & Operation mode of the soaks (101-108) \\
\hline soak_fraction_final & $\begin{array}{l}\text { Sum of the daily average soaks for an individual op-mode, hour, day type, vehicle type, } \\
\text { state and vocation divided by the sum of the daily average starts for individual hour, } \\
\text { day type, vehicle type, state and vocation }\end{array}$ \\
sum_soaks & $\begin{array}{l}\text { Sum of the daily average soaks per op-mode, hour, day type, vehicle type, state and } \\
\text { vocation }\end{array}$ \\
\hline
\end{tabular}

\section{idle_by_state_and_voc_final_102518.csv}

- This file provides the idle fraction for each state, vocation, source type (vehicle type) and day type along with statistics of the data. The full list of parameters included in the file is as follows: 


\begin{tabular}{|c|c|}
\hline Column Name & Description \\
\hline state & State of origin or most frequent operation \\
\hline vocation & Vocation of the vehicle \\
\hline source_type & Source Type \\
\hline day & Day Type $($ weekday=5, weekend $=2)$ \\
\hline idle_fraction_final & $\begin{array}{l}\text { Sum of the daily average idle hours for all vehicles divided by } \\
\text { sum of the daily average operating hours for all vehicles in the } \\
\text { day type, vehicle type, state and vocation }\end{array}$ \\
\hline workday_idle_fraction_final & $\begin{array}{l}\text { Sum of the daily average workday idle hours for all vehicles } \\
\text { divided by sum of the daily average operating hours for all } \\
\text { vehicles in the day type, vehicle type, state and vocation }\end{array}$ \\
\hline extended_idle_fraction_final & $\begin{array}{l}\text { Sum of the daily average extended idle hours for all vehicles } \\
\text { divided by sum of the daily average operating hours for all } \\
\text { vehicles in the day type, vehicle type, state and vocation }\end{array}$ \\
\hline sum_operation_hours & $\begin{array}{l}\text { Sum of the daily average operating hours for all vehicles in the } \\
\text { day type, vehicle type, state and vocation }\end{array}$ \\
\hline sum_idle_hours & $\begin{array}{l}\text { Sum of the daily average idle hours for all vehicles in the day } \\
\text { type, vehicle type, state and vocation }\end{array}$ \\
\hline sum_workday_idle_hours & $\begin{array}{l}\text { Sum of the daily average workday idle hours for all vehicles in } \\
\text { the day type, vehicle type, state and vocation }\end{array}$ \\
\hline sum_extended_idle_hours & $\begin{array}{l}\text { Sum of the daily average extended idle hours for all vehicles in } \\
\text { the day type, vehicle type, state and vocation }\end{array}$ \\
\hline sum_miles_driven & $\begin{array}{l}\text { Sum of the average daily miles driven for that day type, vehicle } \\
\text { type, state and vocation }\end{array}$ \\
\hline extended_idle_hours/miles_driven & $\begin{array}{l}\text { Sum of the daily average extended idle hours for all vehicles } \\
\text { divided by sum of the daily average miles traveled for all vehicles } \\
\text { in the day and vehicle type }\end{array}$ \\
\hline population & $\begin{array}{l}\text { Population of vehicles for that day type, vehicle type, state and } \\
\text { vocation }\end{array}$ \\
\hline
\end{tabular}

\section{Results for Individual Vehicles File List}

\section{idle_by_state_and_voc_individual_veh_all_102518.csv}

- This file provides the idle fraction for each state, vocation, source type (vehicle type) and day type along with statistics of the data. The full list of parameters included in the file is as follows:

$\bullet$

\begin{tabular}{|l|l|}
\hline Column Name & Description \\
\hline vid & Unique Vehicle ID \\
\hline state & State of origin or most frequent operation \\
\hline vocation & Vocation of the vehicle \\
\hline source_type & Source Type \\
\hline
\end{tabular}




\begin{tabular}{|l|l|} 
day & Day Type (weekday=5, weekend = 2) \\
\hline idle_fraction & Daily average idle hours for the vehicle \\
\hline workday_idle_fraction & Daily average workday idle hours for the vehicle \\
\hline extended_idle_fraction & Daily average extended idle hours for the vehicle \\
\hline operation_hours & Daily average operating hours for the vehicle \\
\hline idle_hours & Daily average idle hours for the vehicle \\
\hline workday_idle_hours & Daily average workday idle hours for the vehicle \\
\hline extended_idle_hours & Daily average extended idle hours for the vehicle \\
\hline miles_driven & Daily average miles driven for the vehicle \\
\hline extended_idle_hours/miles_driven & $\begin{array}{l}\text { Daily average extended idle hours divided by the daily average } \\
\text { miles traveled for the vehicle }\end{array}$ \\
\hline avg_starts_per_day & Average starts per day for the vehicle \\
\hline average_soak_length_minutes & Average soak length for the vehicle \\
\hline
\end{tabular}




\section{Appendix E: Daily Start and Idle Distributions}

\section{Distributions of Daily Starts by Vehicle Type}
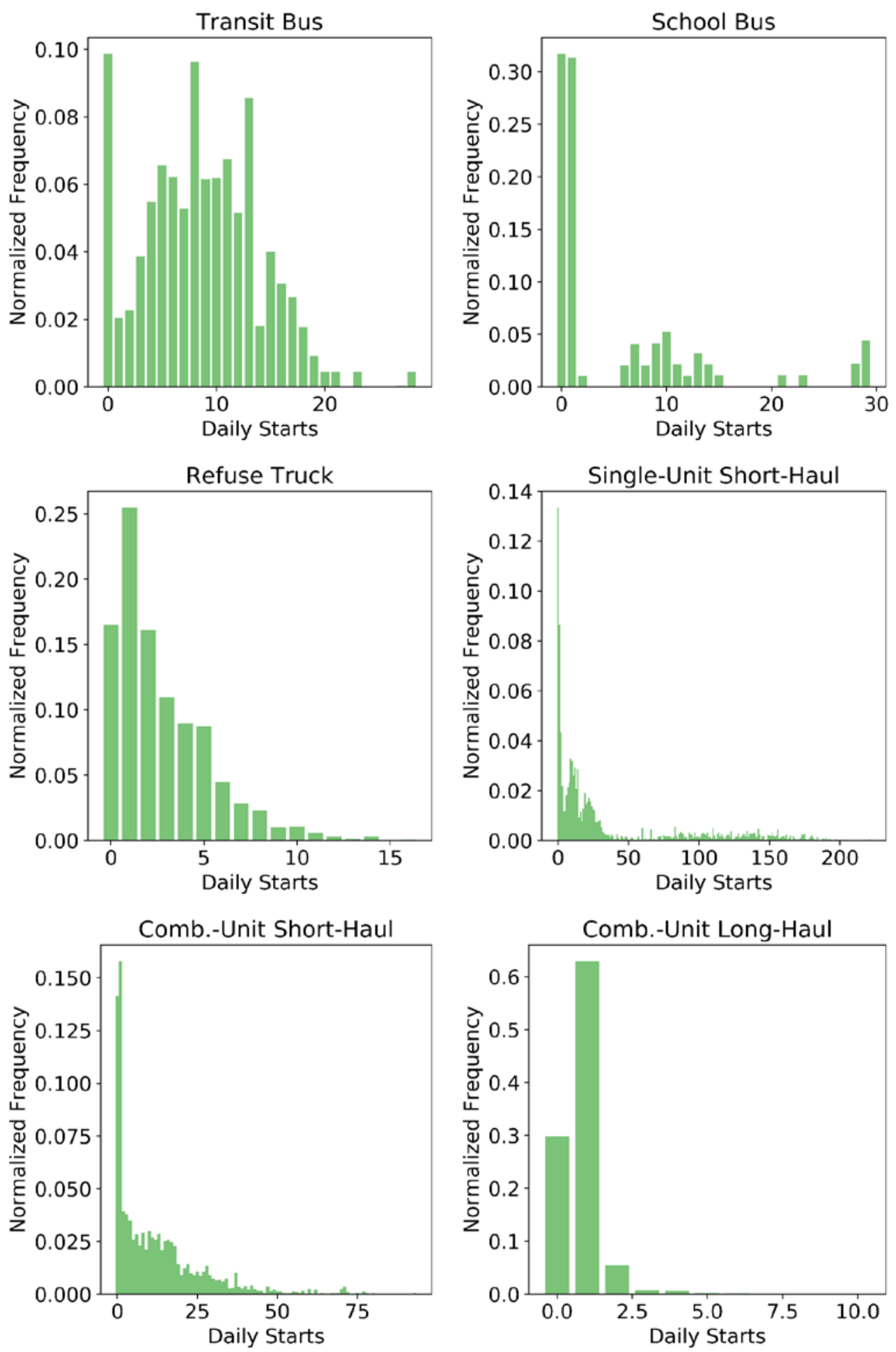


\section{Distributions of Idle Fraction by Vehicle Type and Idle Type}
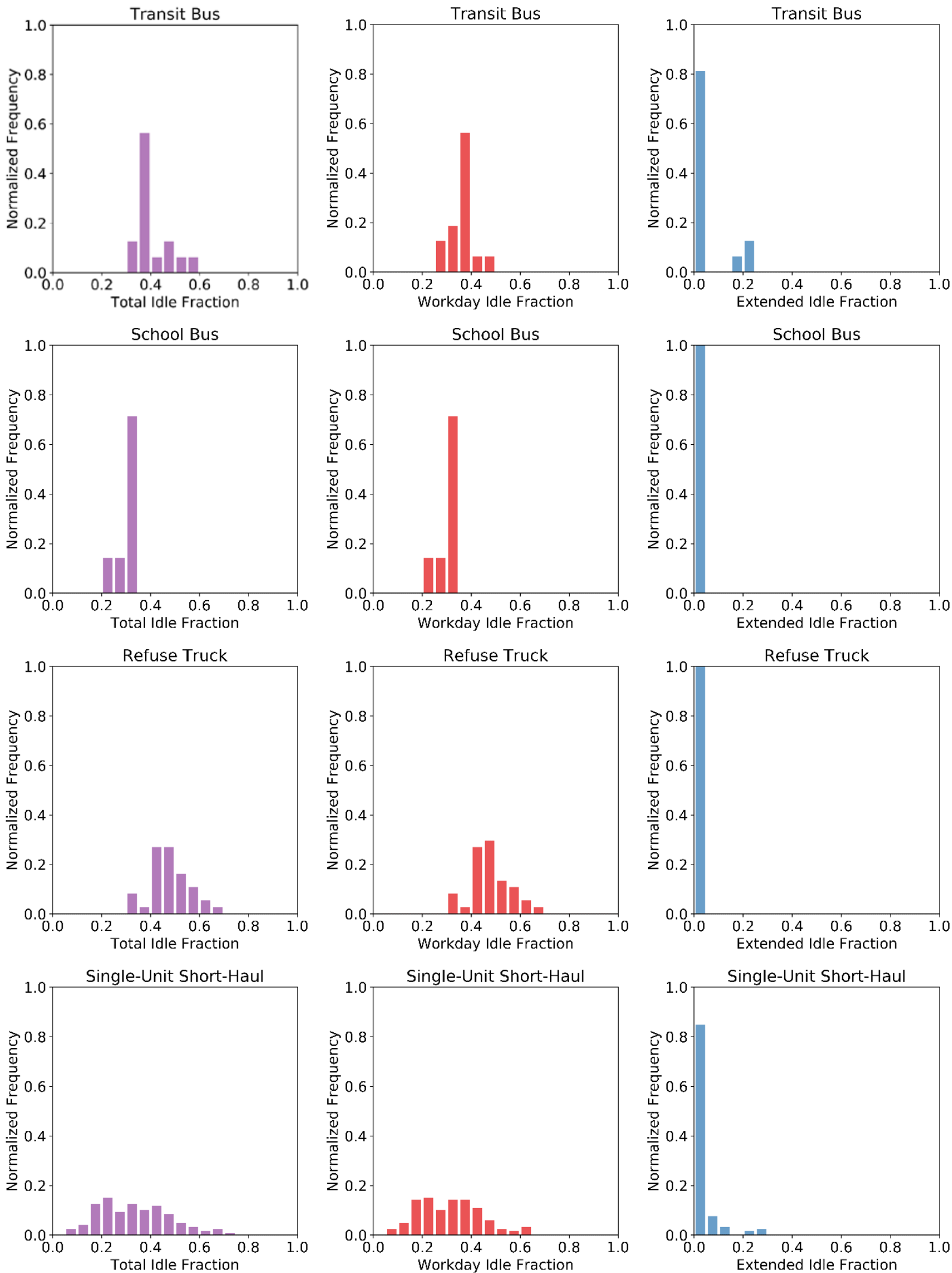

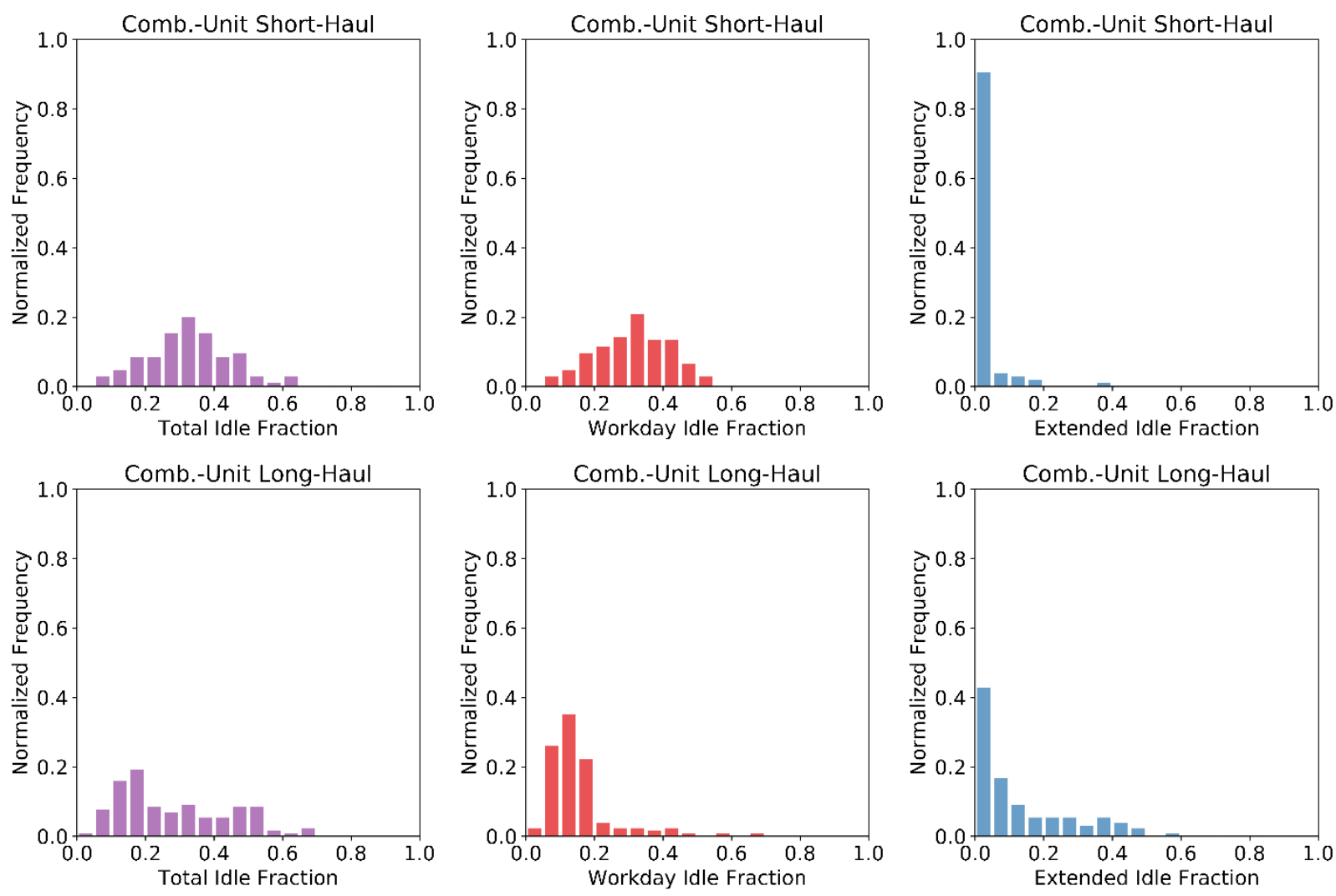


\section{Appendix F: Result Plots}
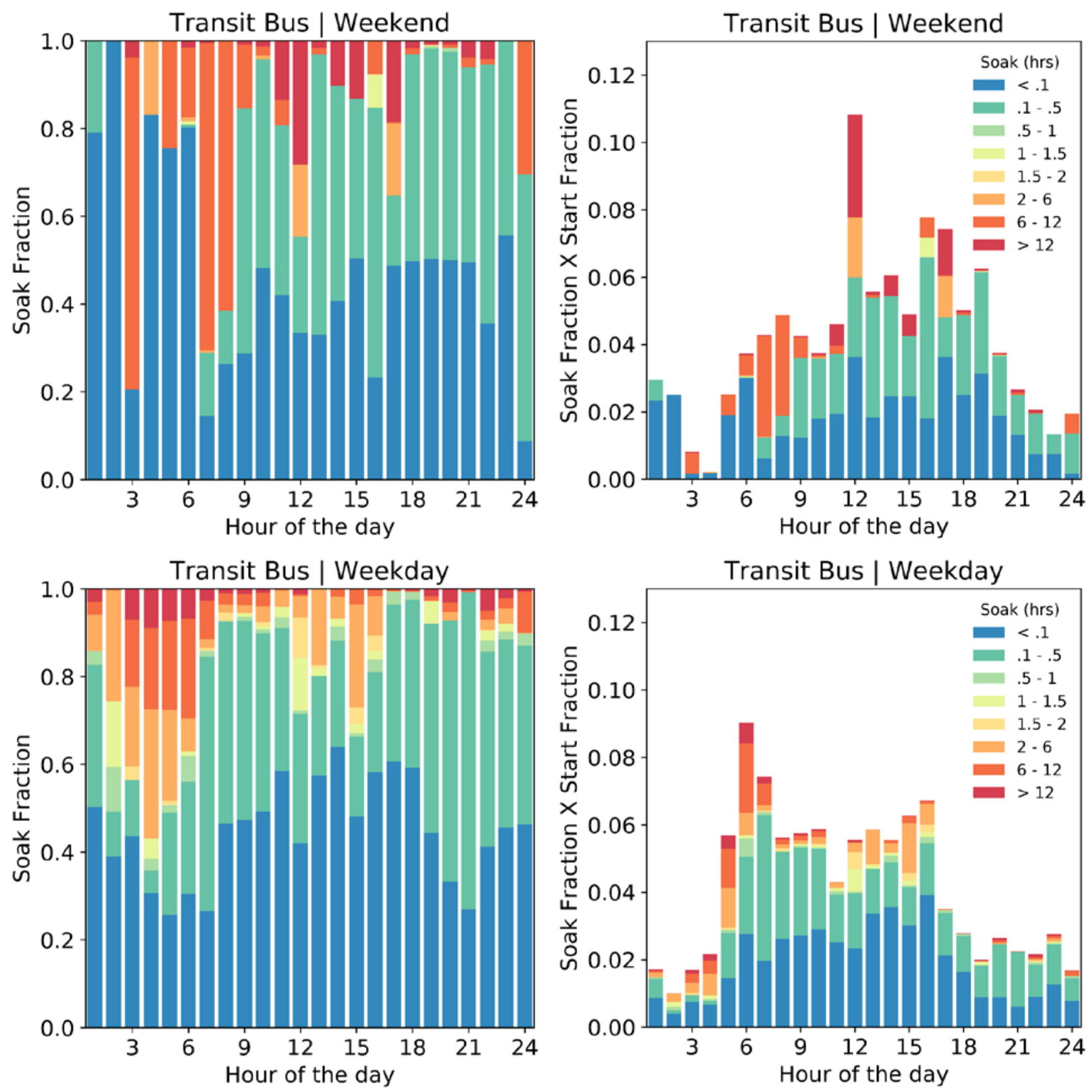

Figure F.1. Soak fraction and start fraction vs. hour of the day for Transit Bus 

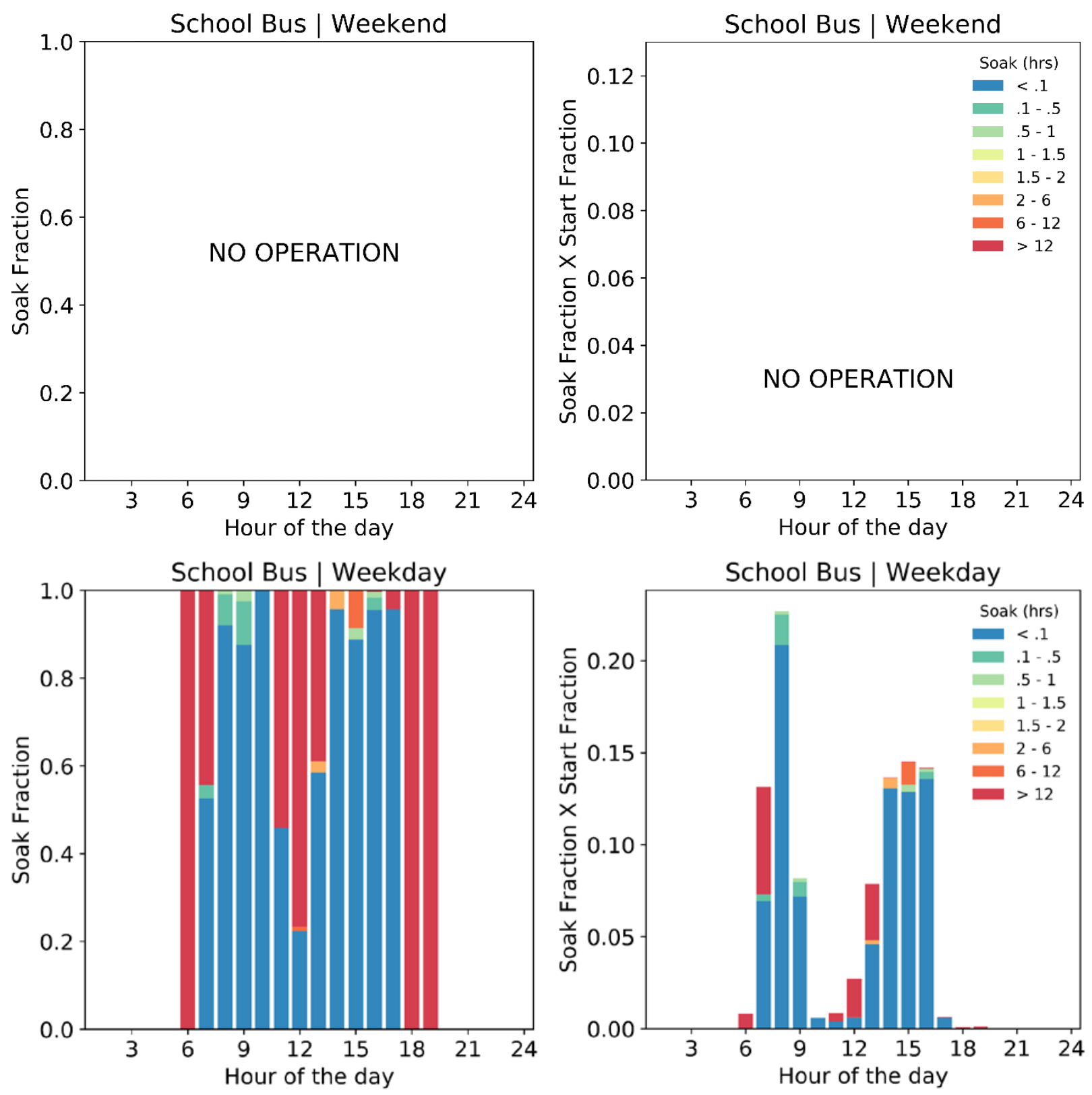

Figure F.2. Soak fraction and start fraction vs. hour of the day for School Bus 

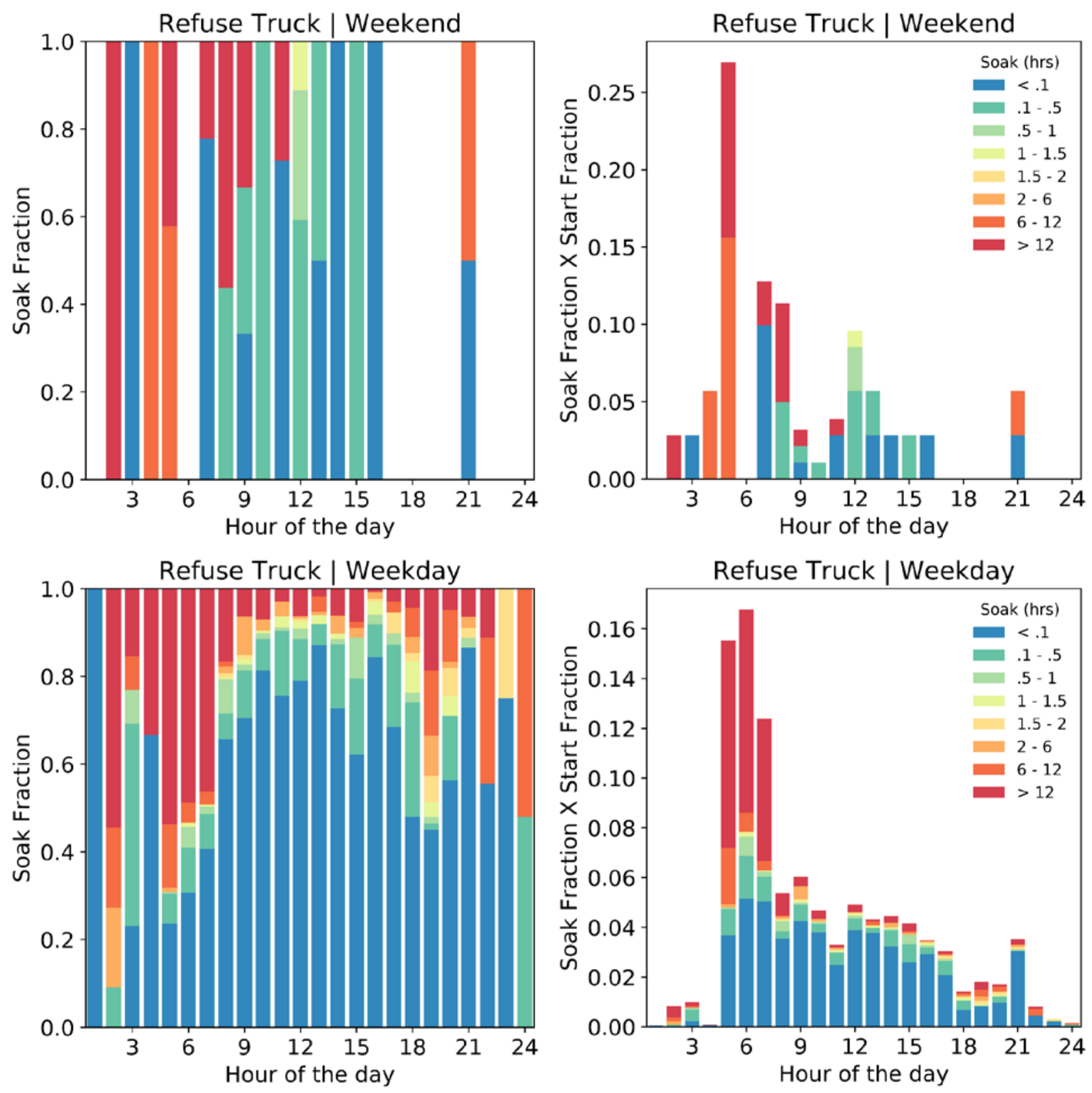

Figure F.3. Soak fraction and start fraction vs. hour of the day for Refuse Truck 

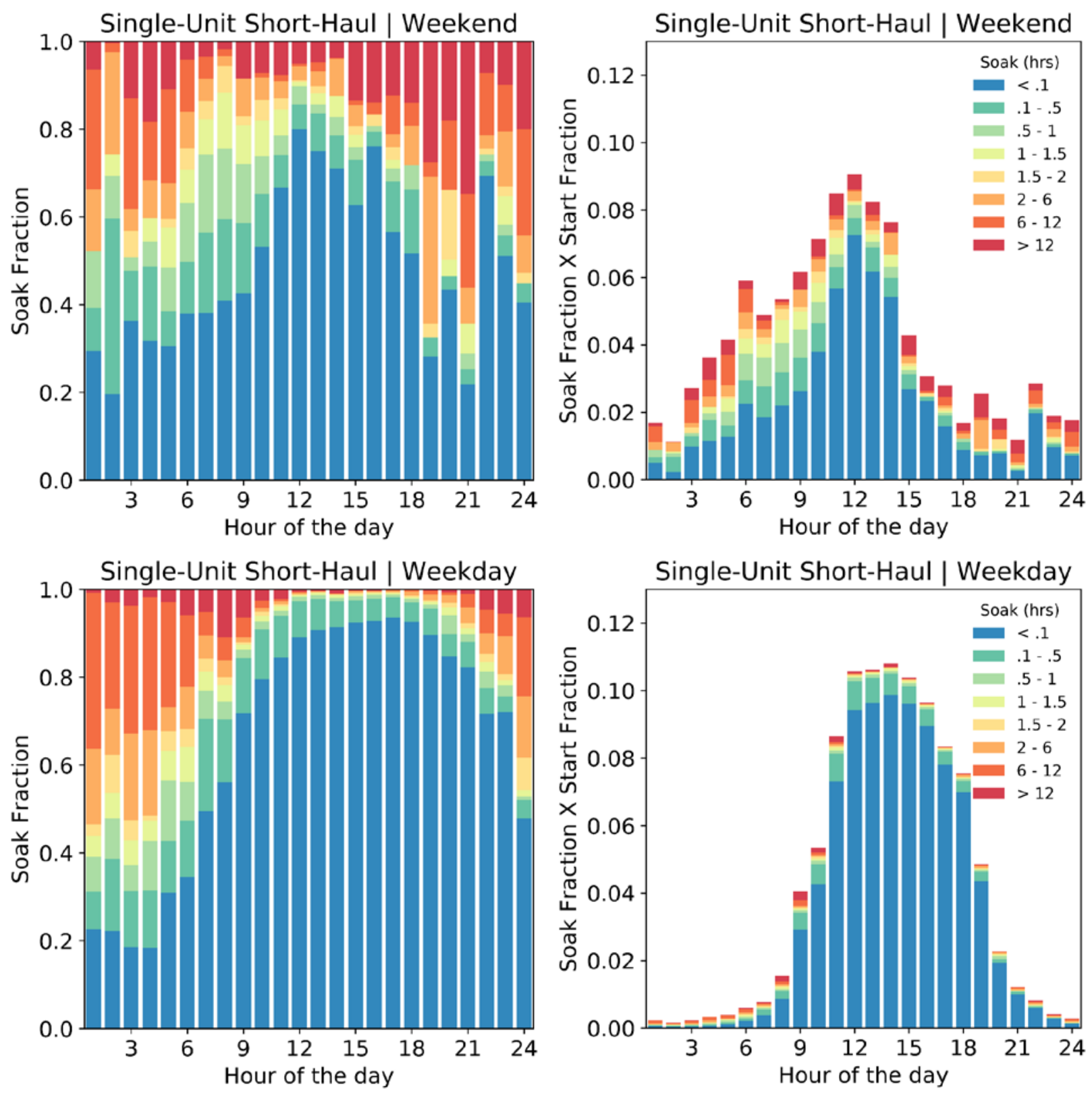

Figure F.4. Soak fraction and start fraction vs. hour of the day for Single-Unit Short-Haul 

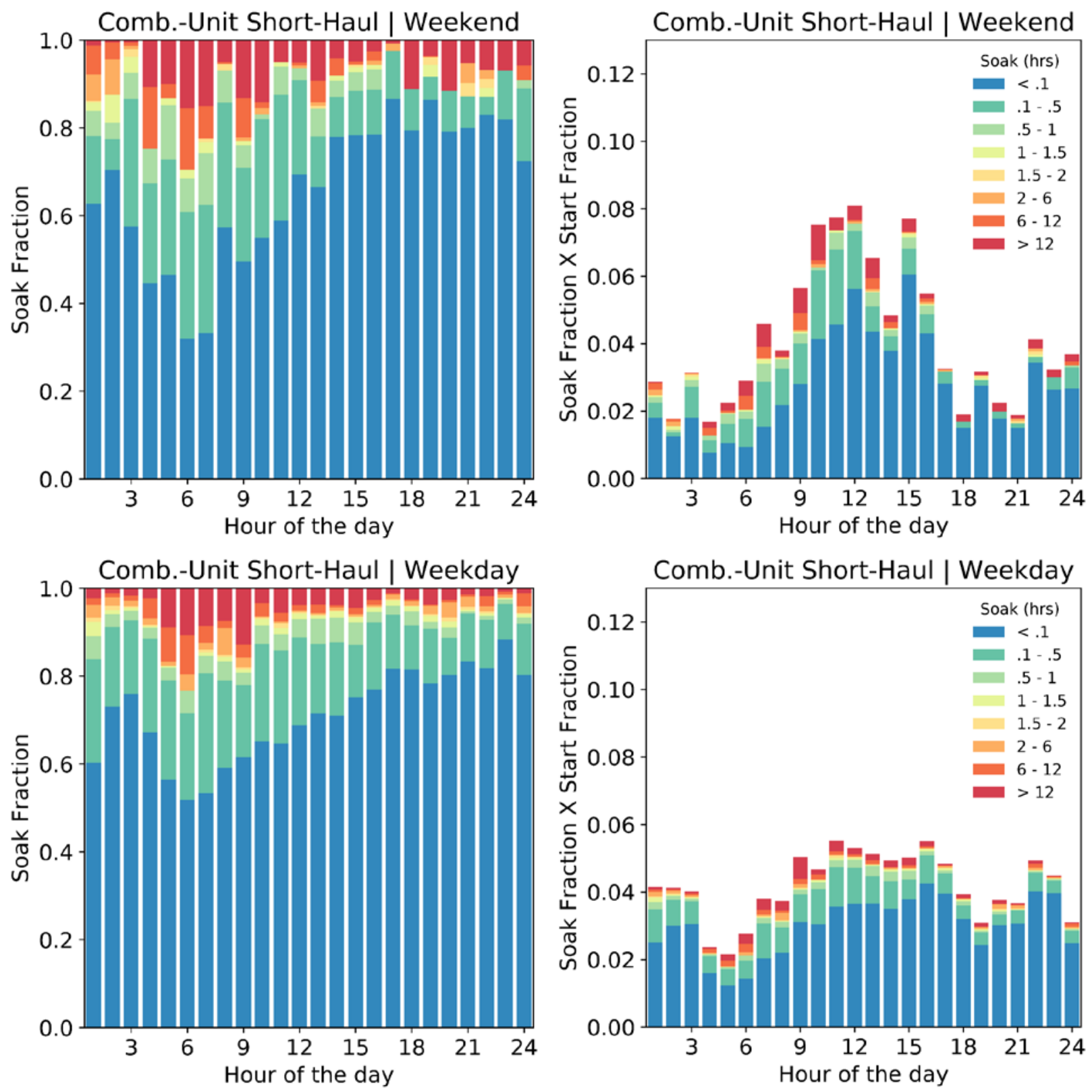

Figure F.5. Soak fraction and start fraction vs. hour of the day for Comb.-Unit Short-Haul 

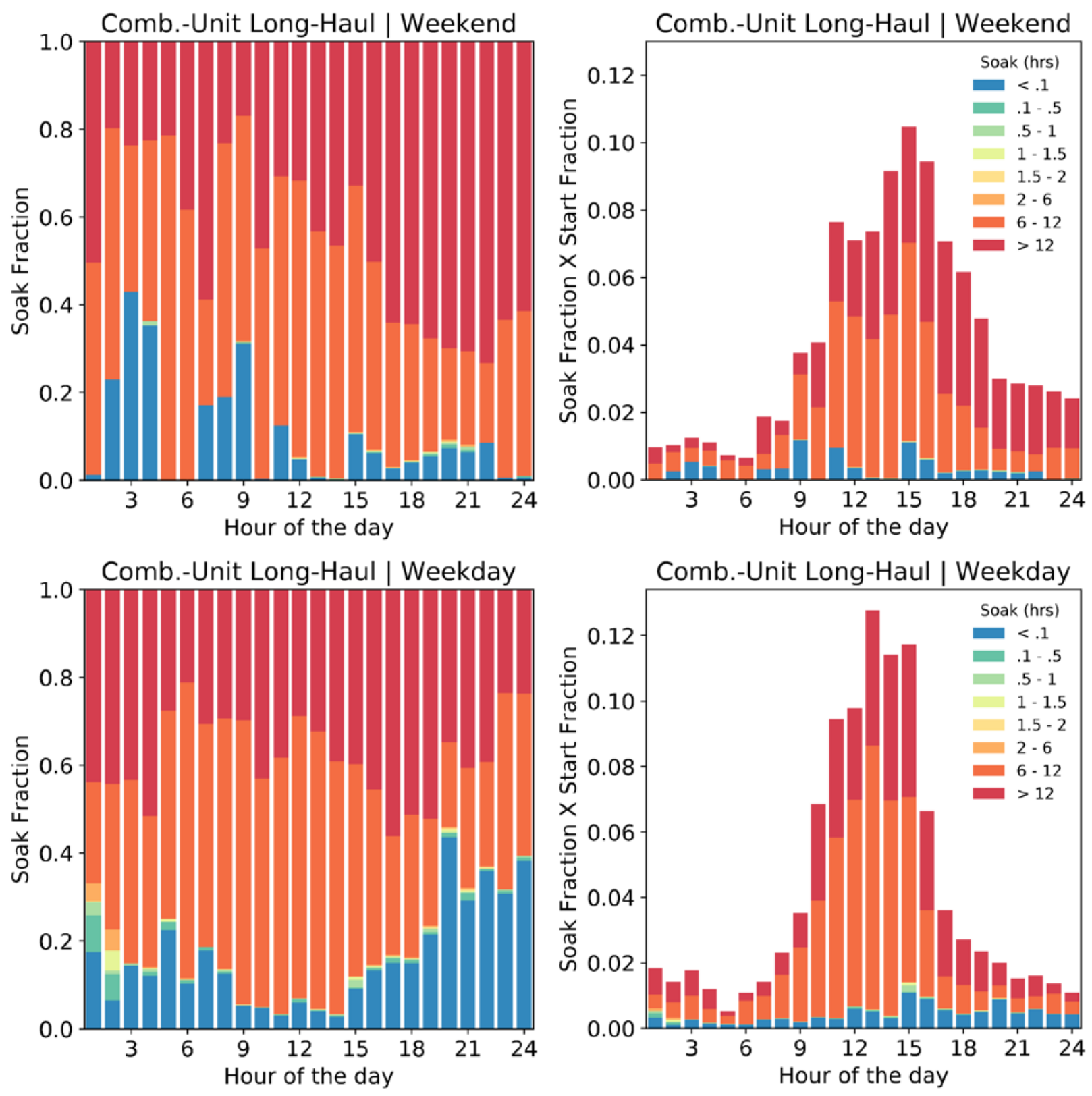

Figure F.6. Soak fraction and start fraction vs. hour of the day for Comb.-Unit Long-Haul 


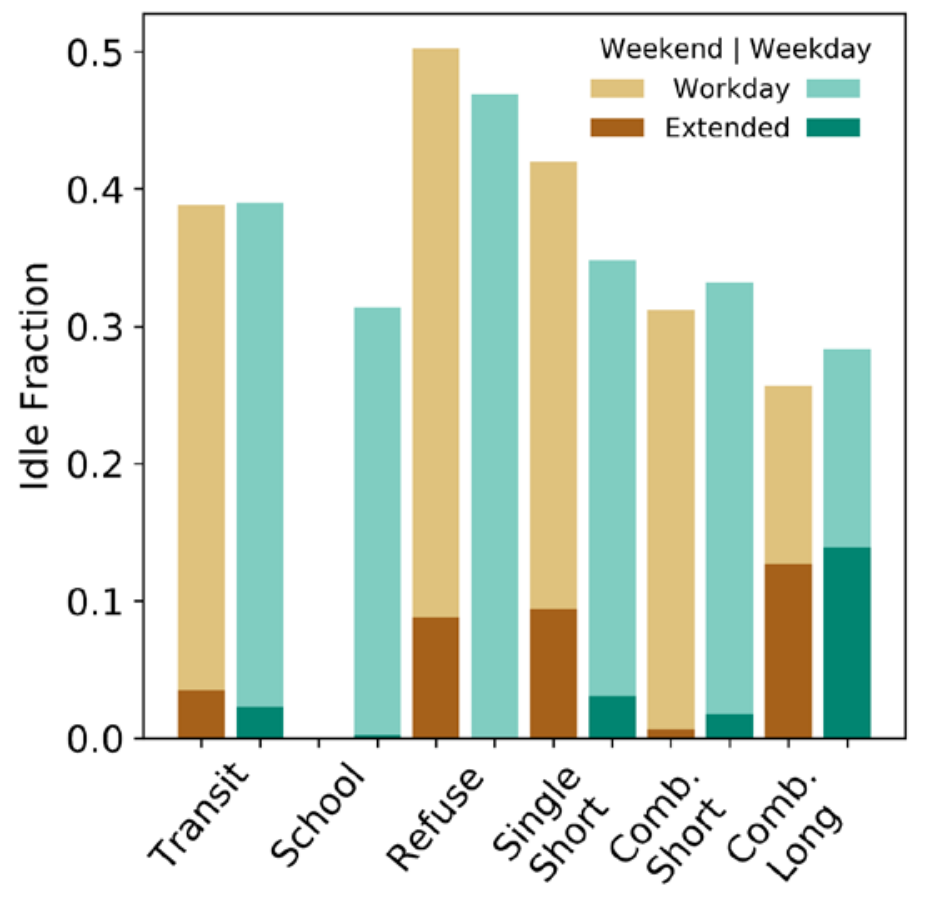

Figure F.7. Extended and workday idle fractions vs. day type and vehicle type 


\section{Appendix G: Hourly Idle Plots}
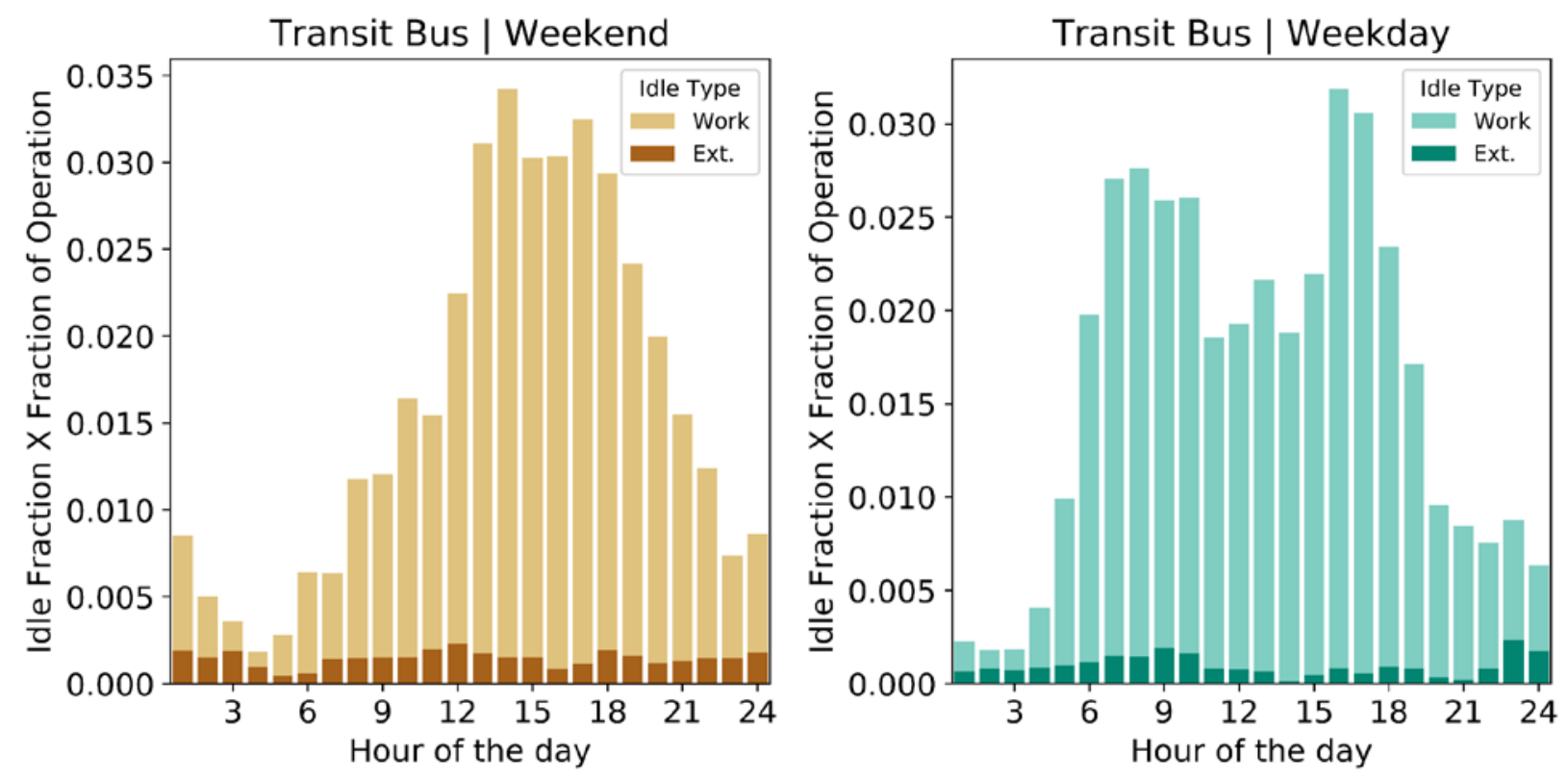

Figure G.1. Hourly idle fraction vs. hour of the day for Transit Bus
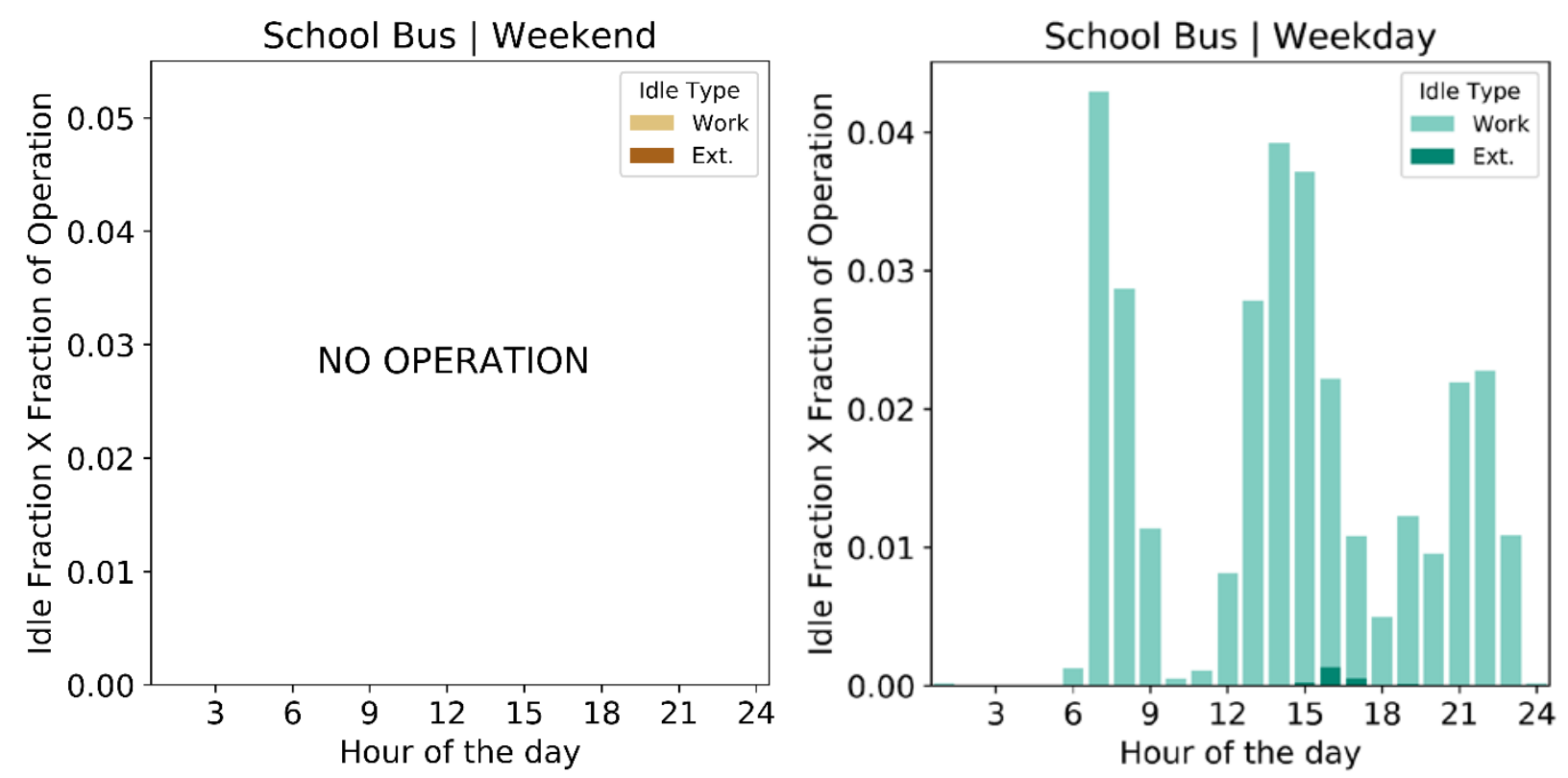

Figure G.2. Hourly idle fraction vs. hour of the day for School Bus 

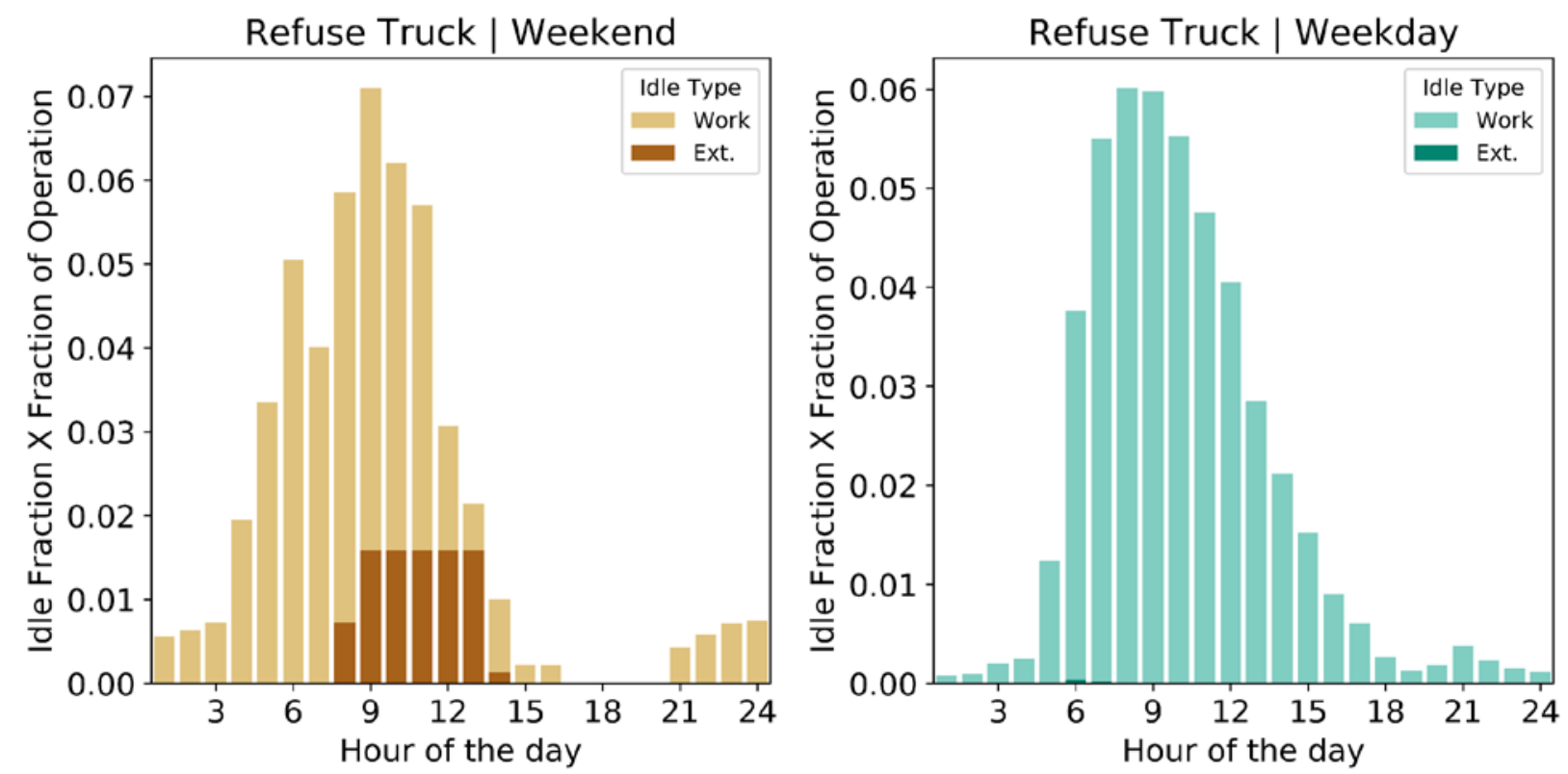

Figure G.3. Hourly idle fraction vs. hour of the day for Refuse Truck
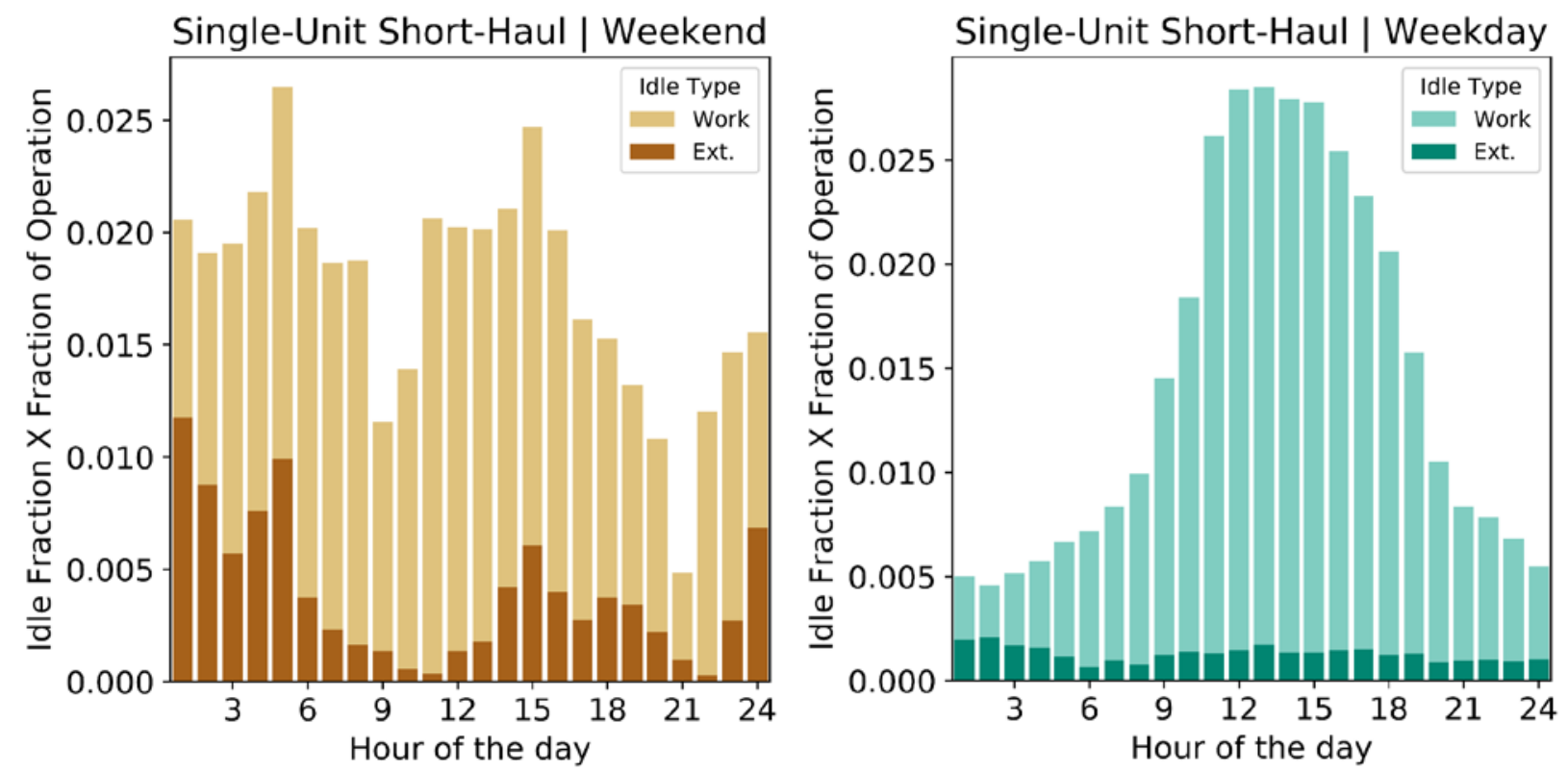

Figure G.4. Hourly idle fraction vs. hour of the day for Single-Unit Short-Haul 

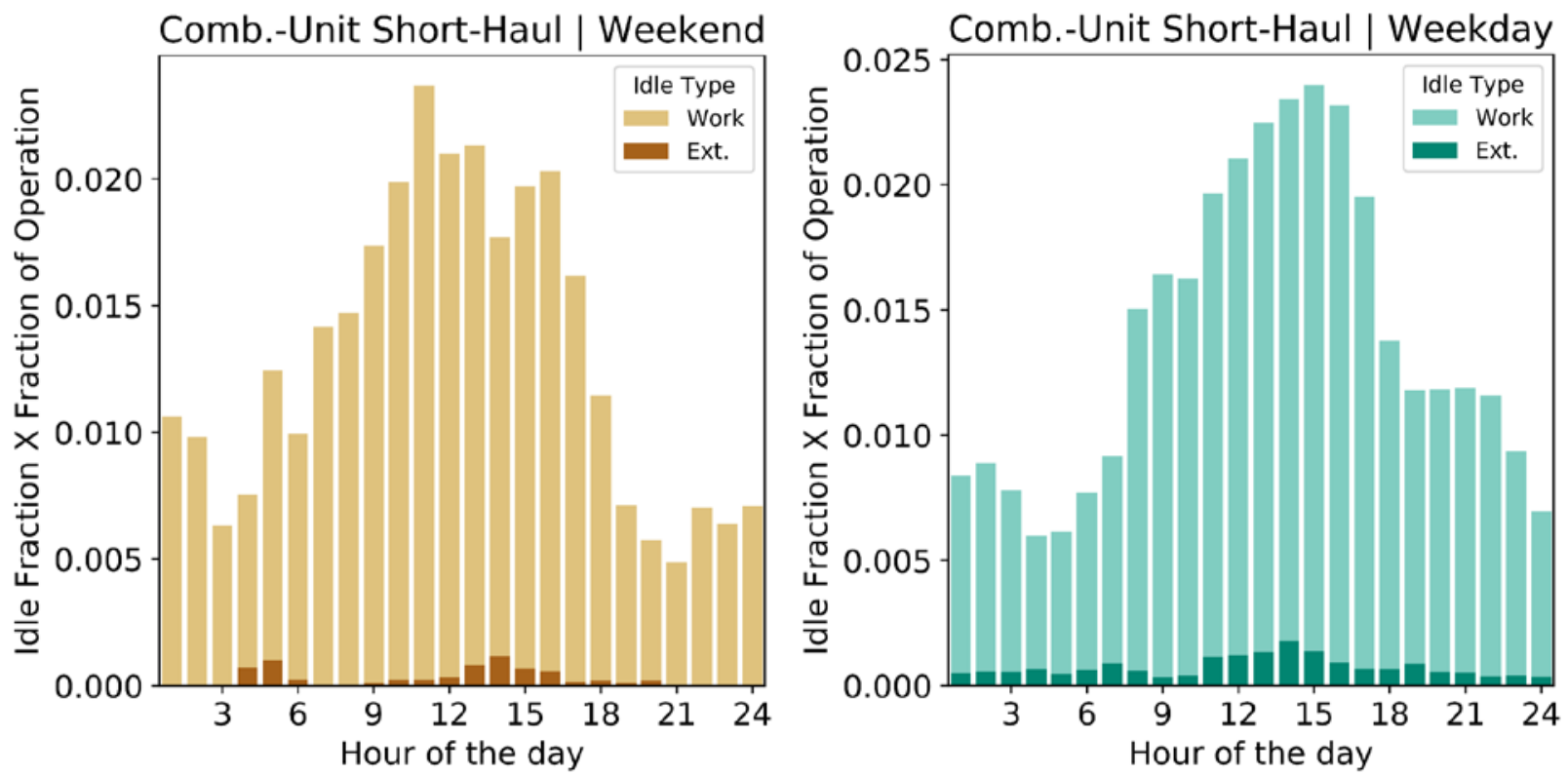

Figure G.5. Hourly idle fraction vs. hour of the day for Comb.-Unit Short-Haul
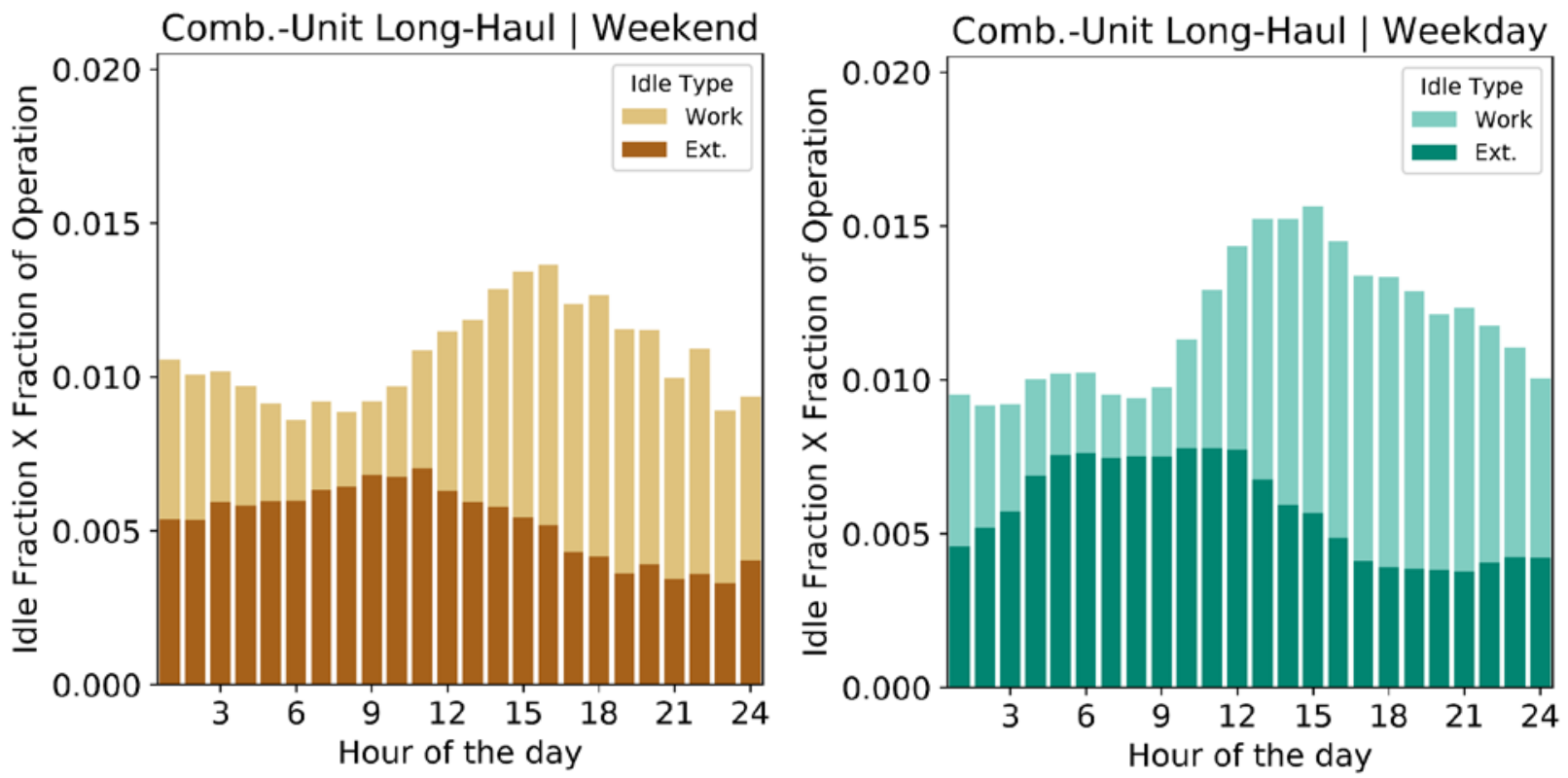

Figure G.6. Hourly idle fraction vs. hour of the day for Comb.-Unit Long-Haul 


\section{Appendix H: Result by State}

\section{Transit Bus}
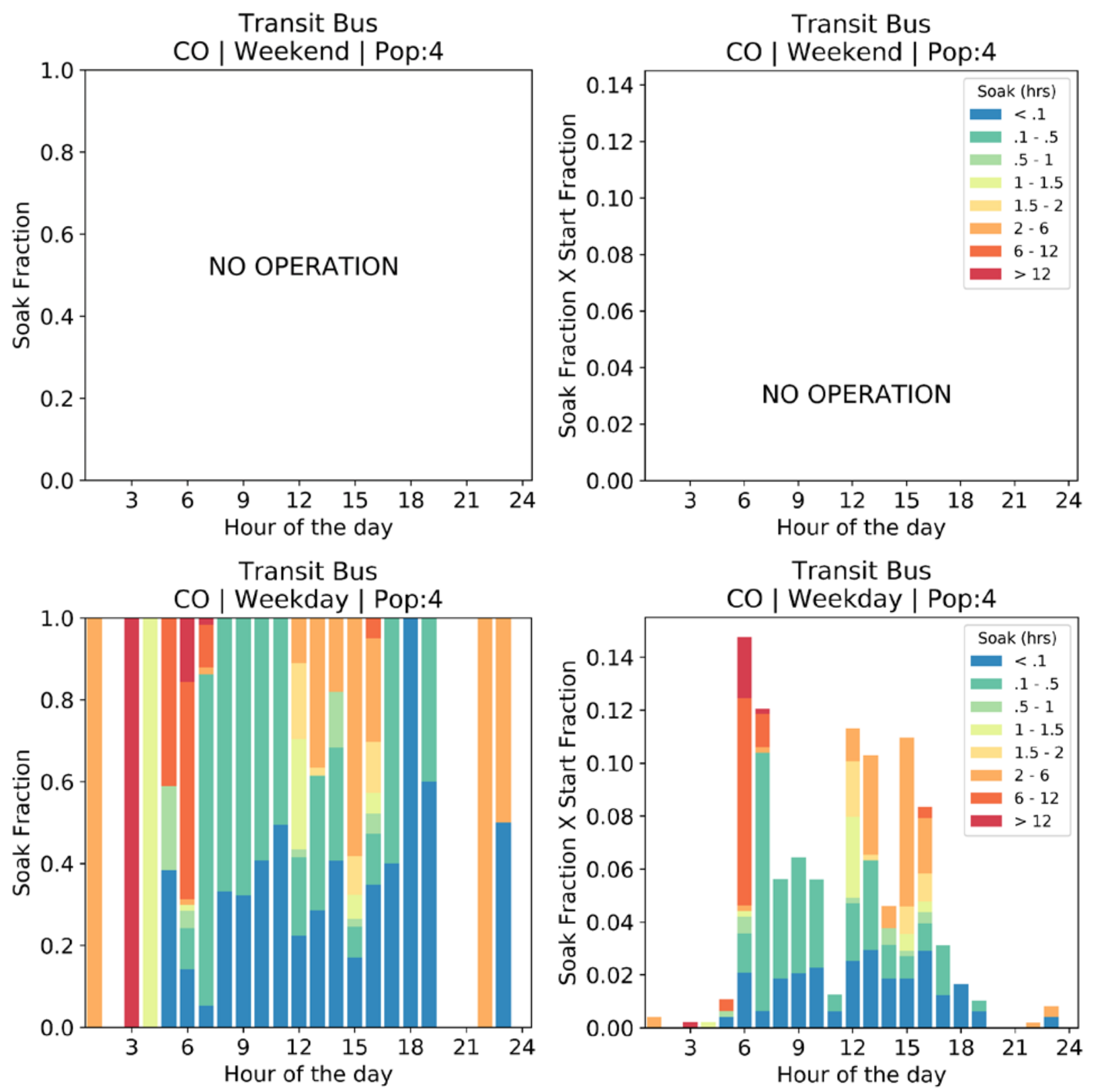

Figure H.1. Soak fraction and start fraction vs. hour of the day for Transit Bus - CO 

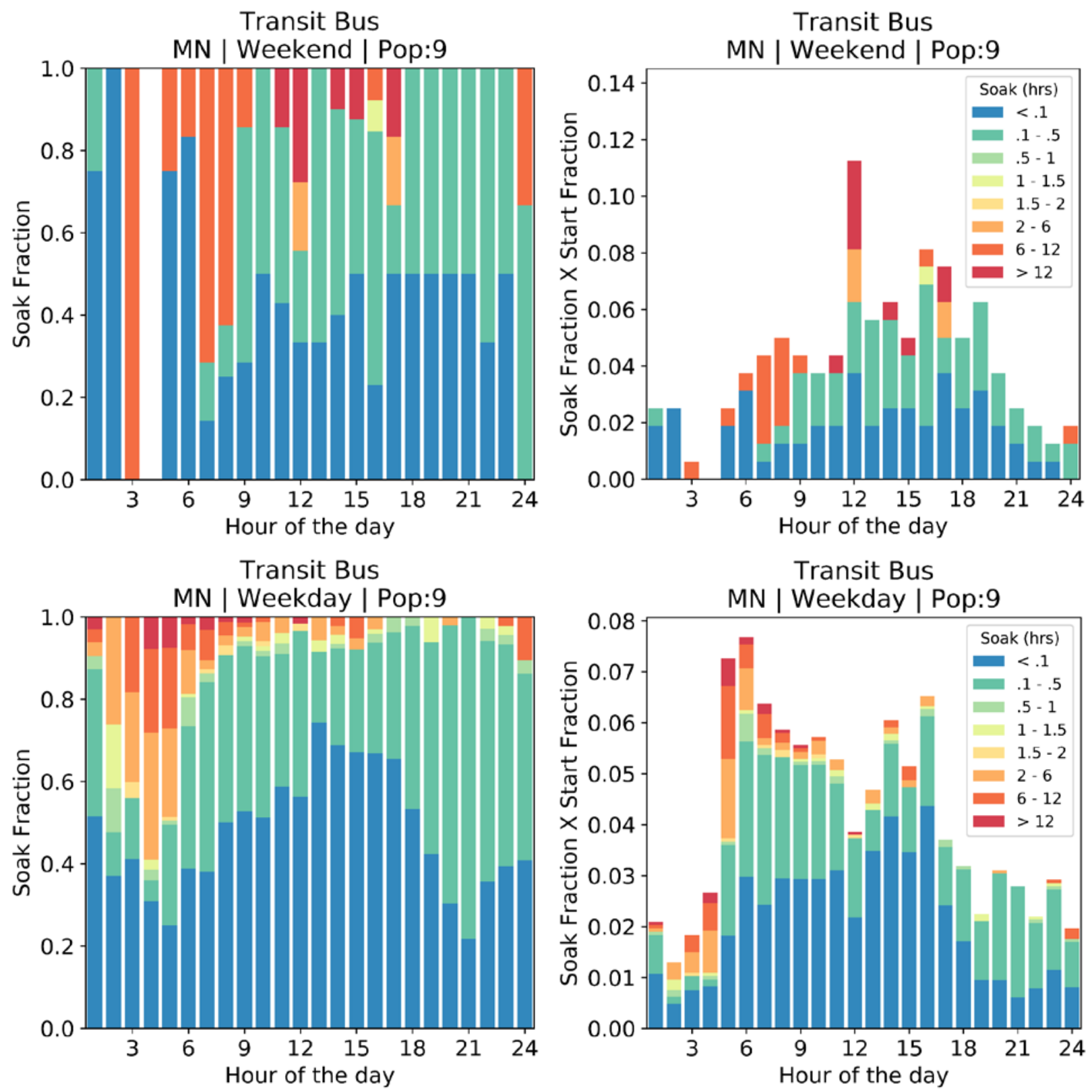

Figure H.2. Soak fraction and start fraction vs. hour of the day for Transit Bus - MN 

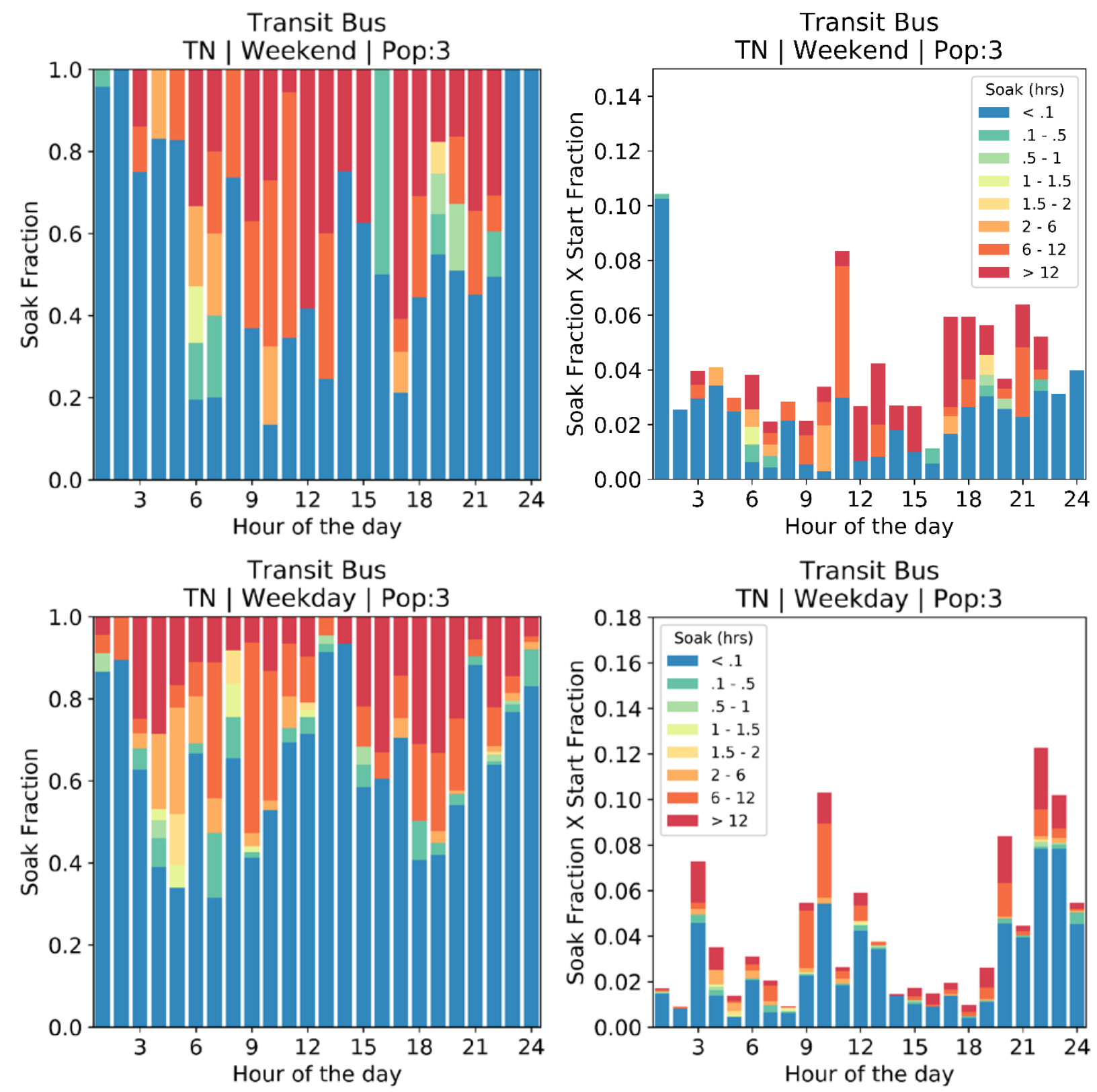

Figure H.3. Soak fraction and start fraction vs. hour of the day for Transit Bus - TN 


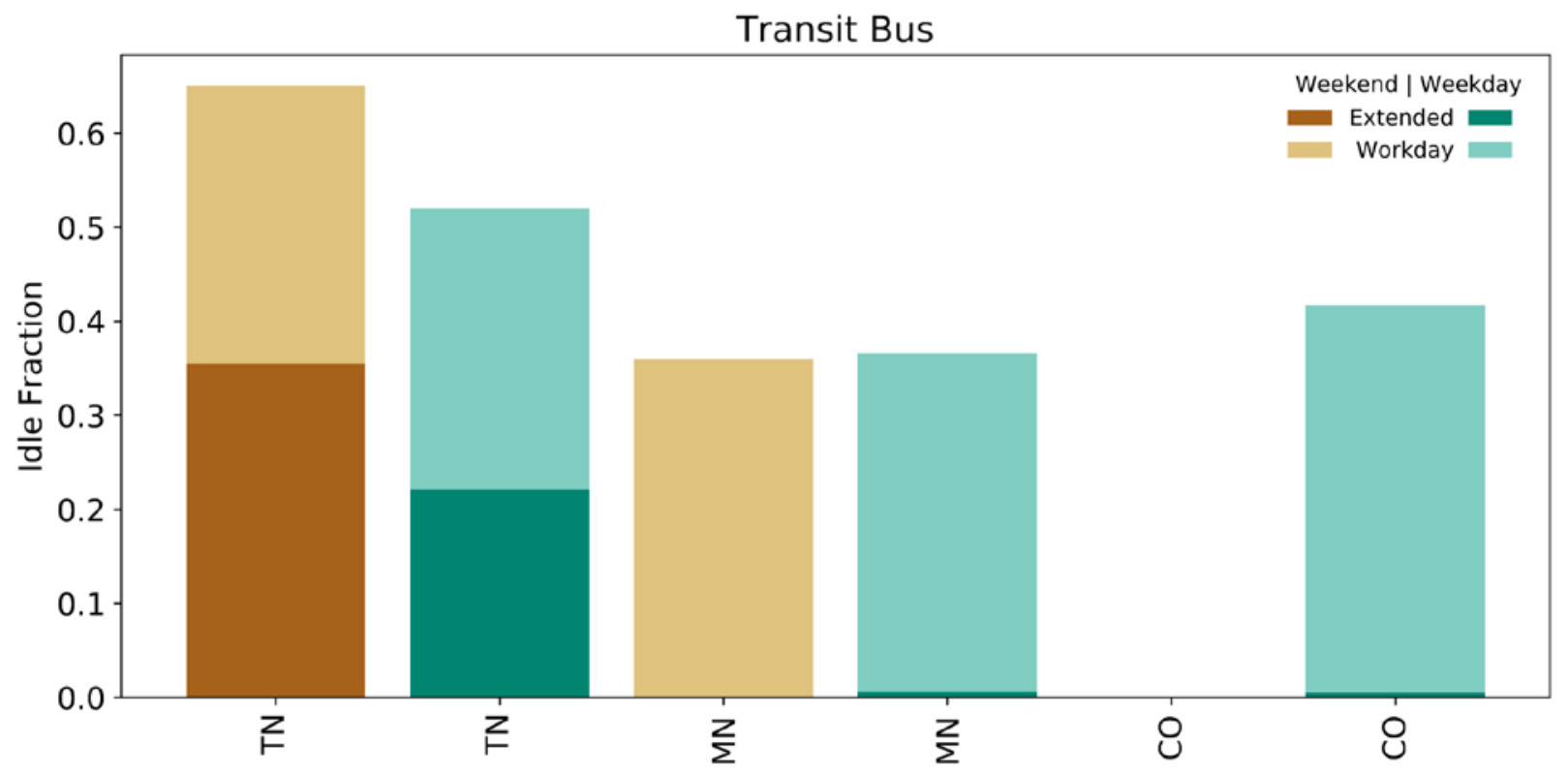

Figure H.4. Extended and workday idle fractions vs. day type and state for Transit Bus 
School Bus

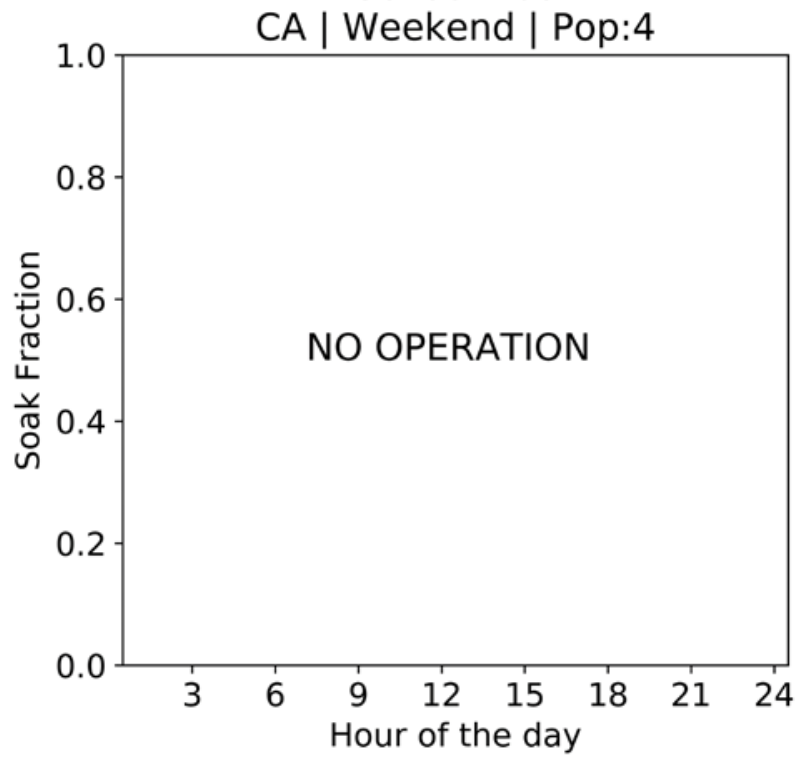

School Bus

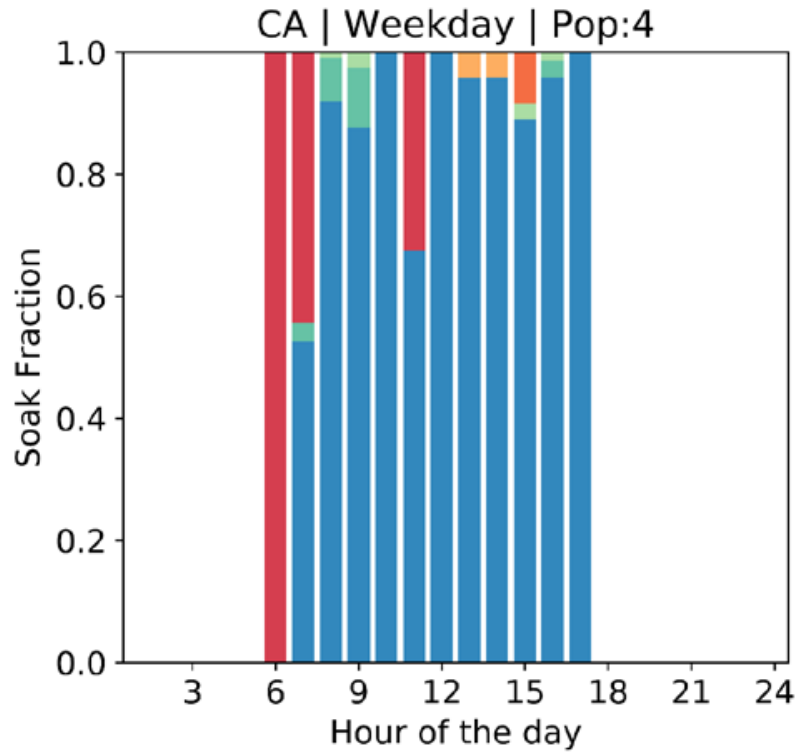

School Bus

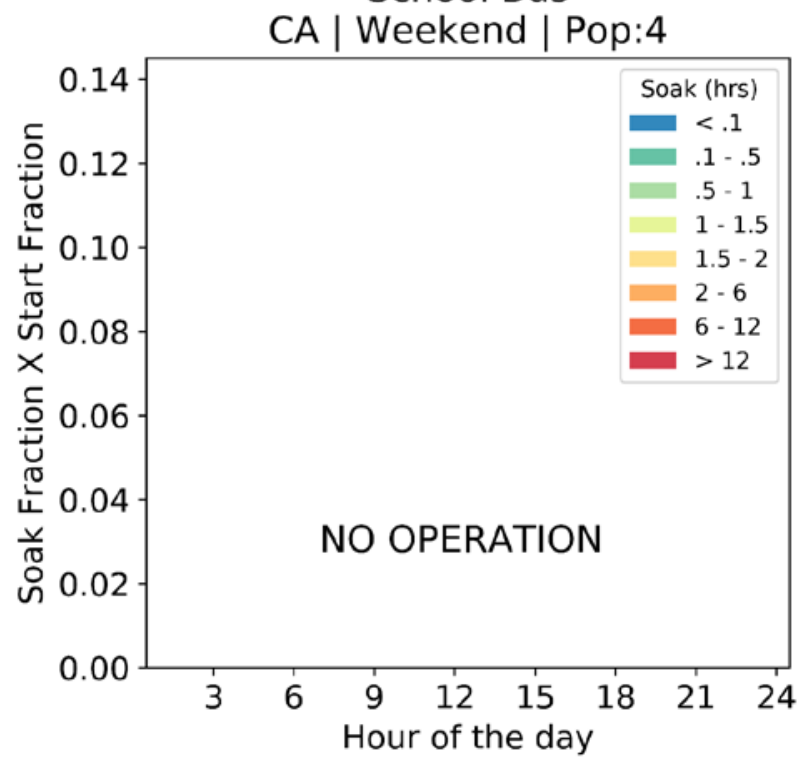

School Bus

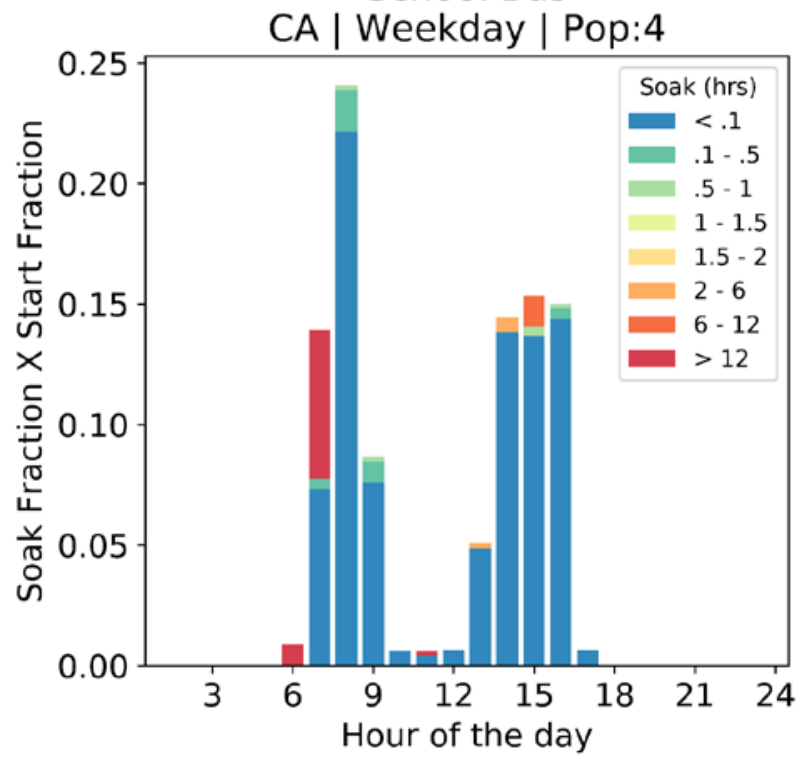

Figure H.5. Soak fraction and start fraction vs. hour of the day for School Bus - CA 
School Bus

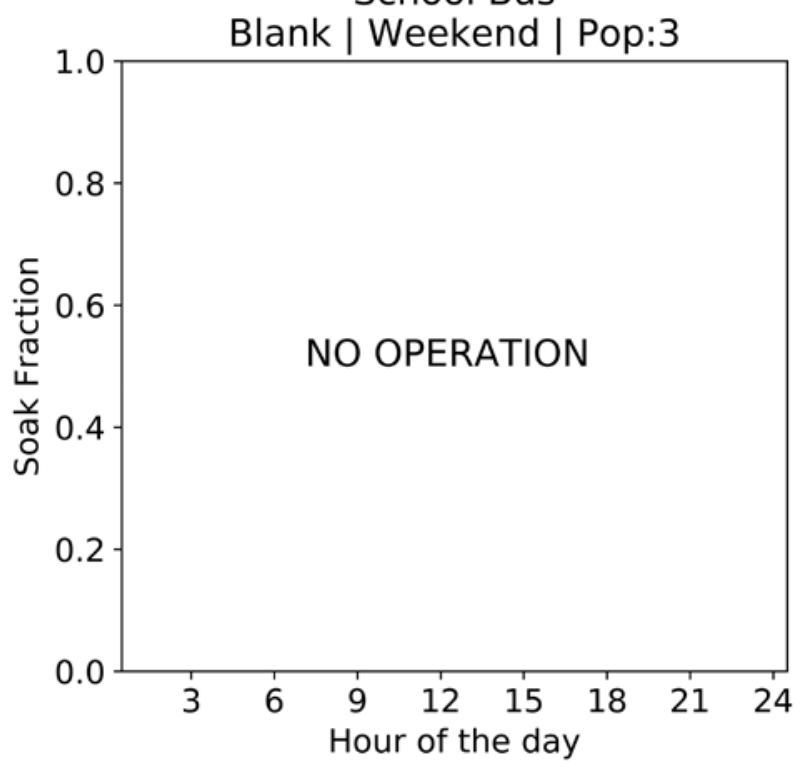

School Bus

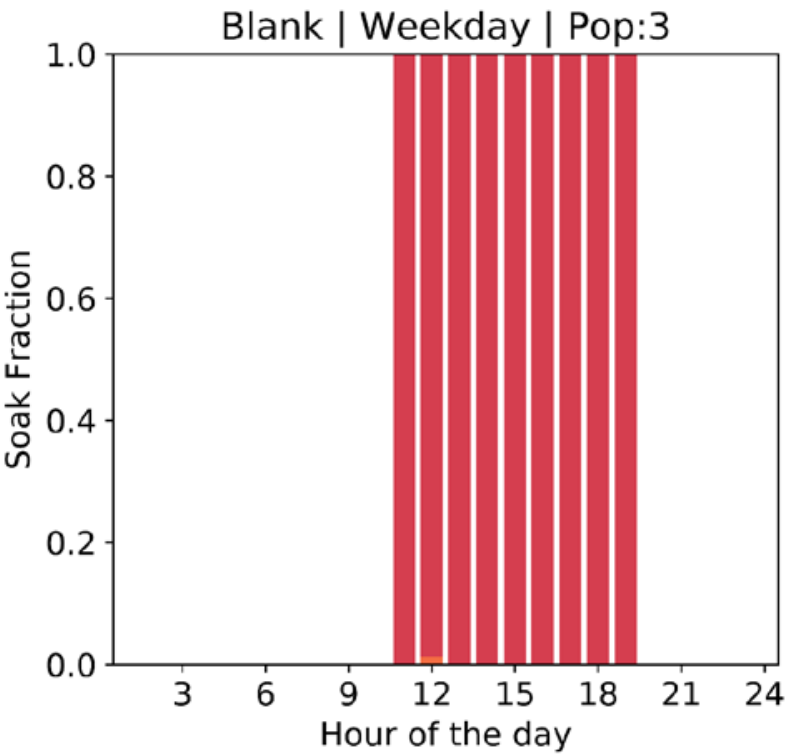

School Bus

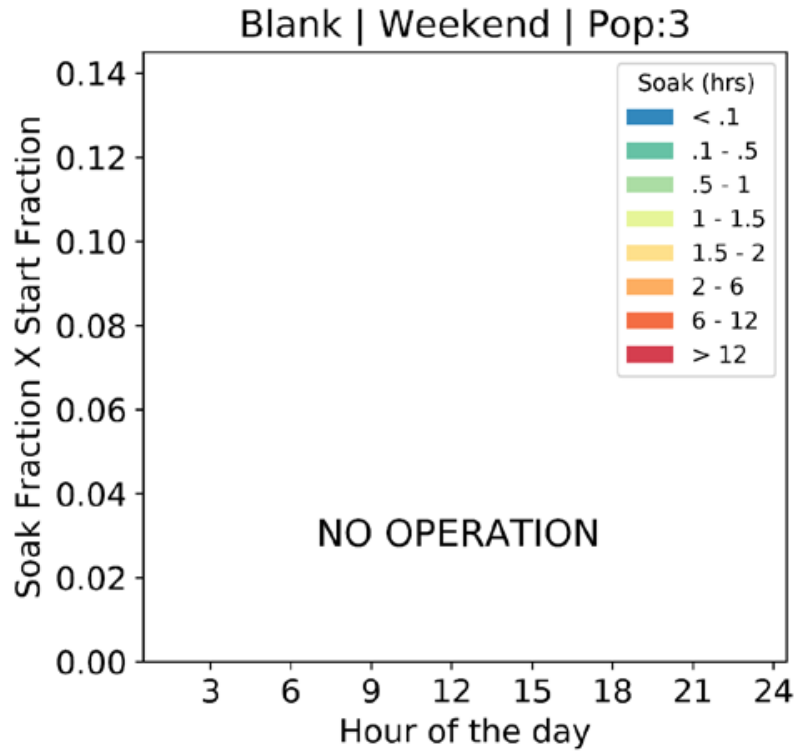

School Bus

Blank | Weekday | Pop:3

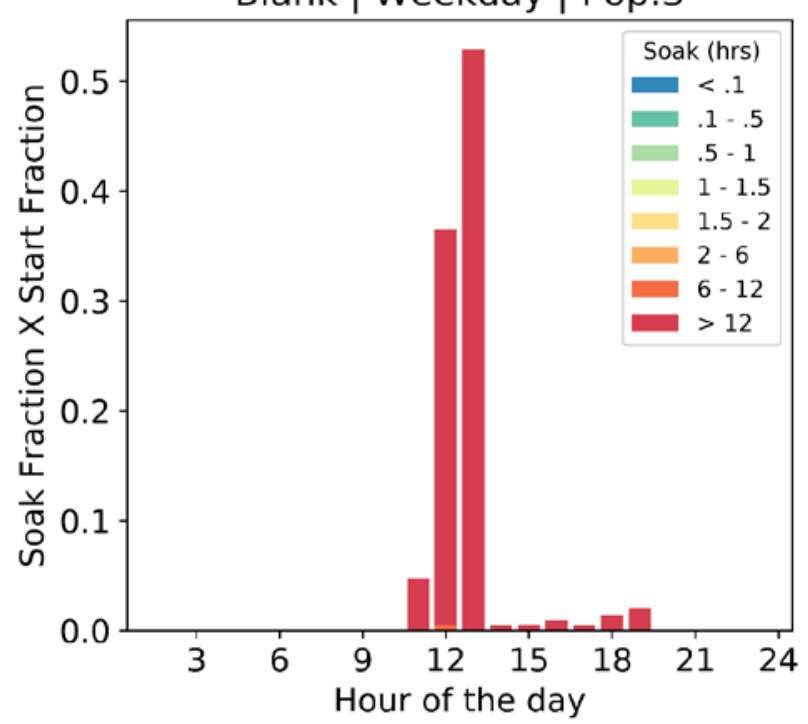

Figure H.6. Soak fraction and start fraction vs. hour of the day for School Bus - Blank 


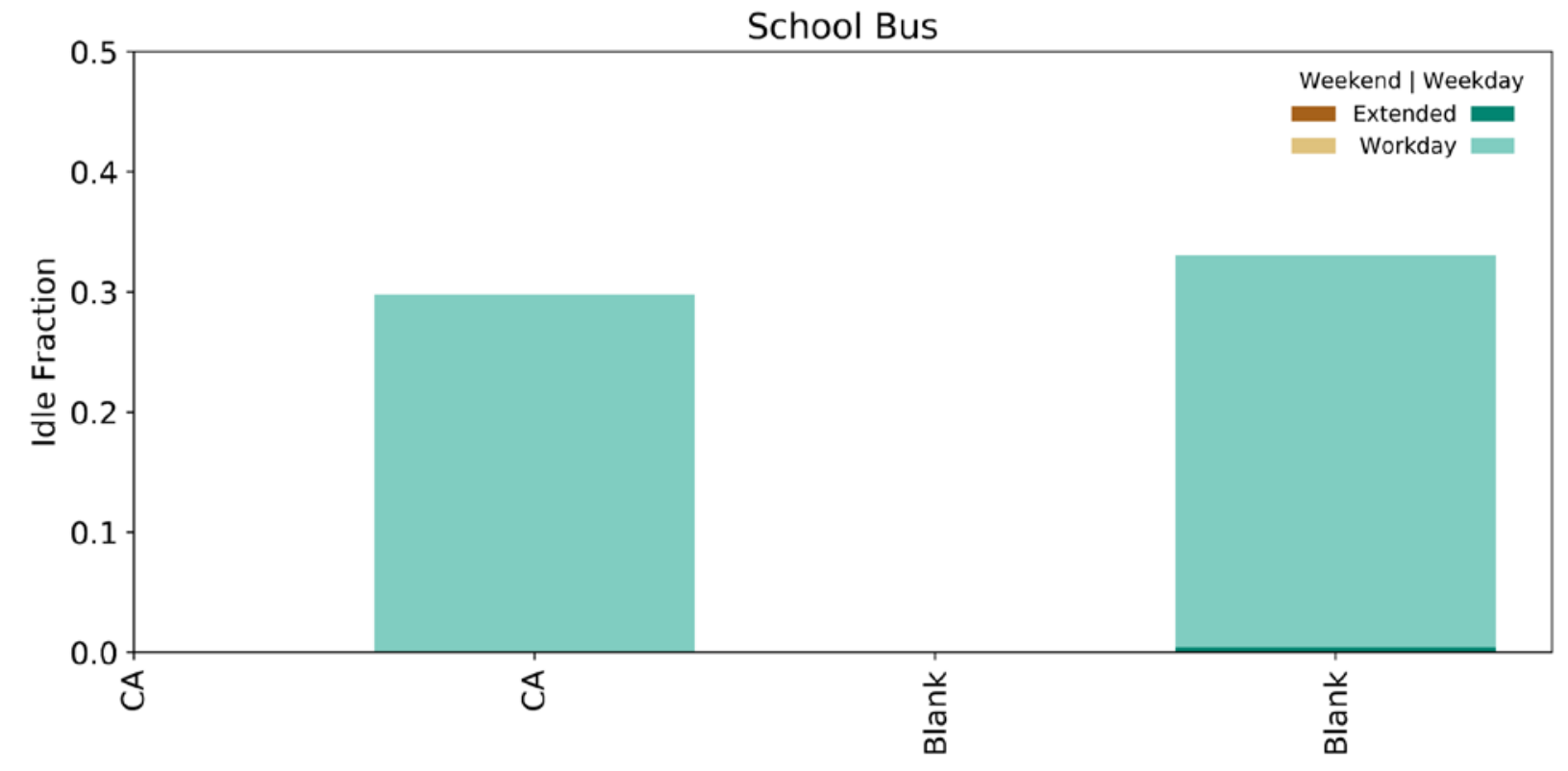

Figure H.7. Extended and workday idle fractions vs. day type and state for School Bus 


\section{Refuse Truck}
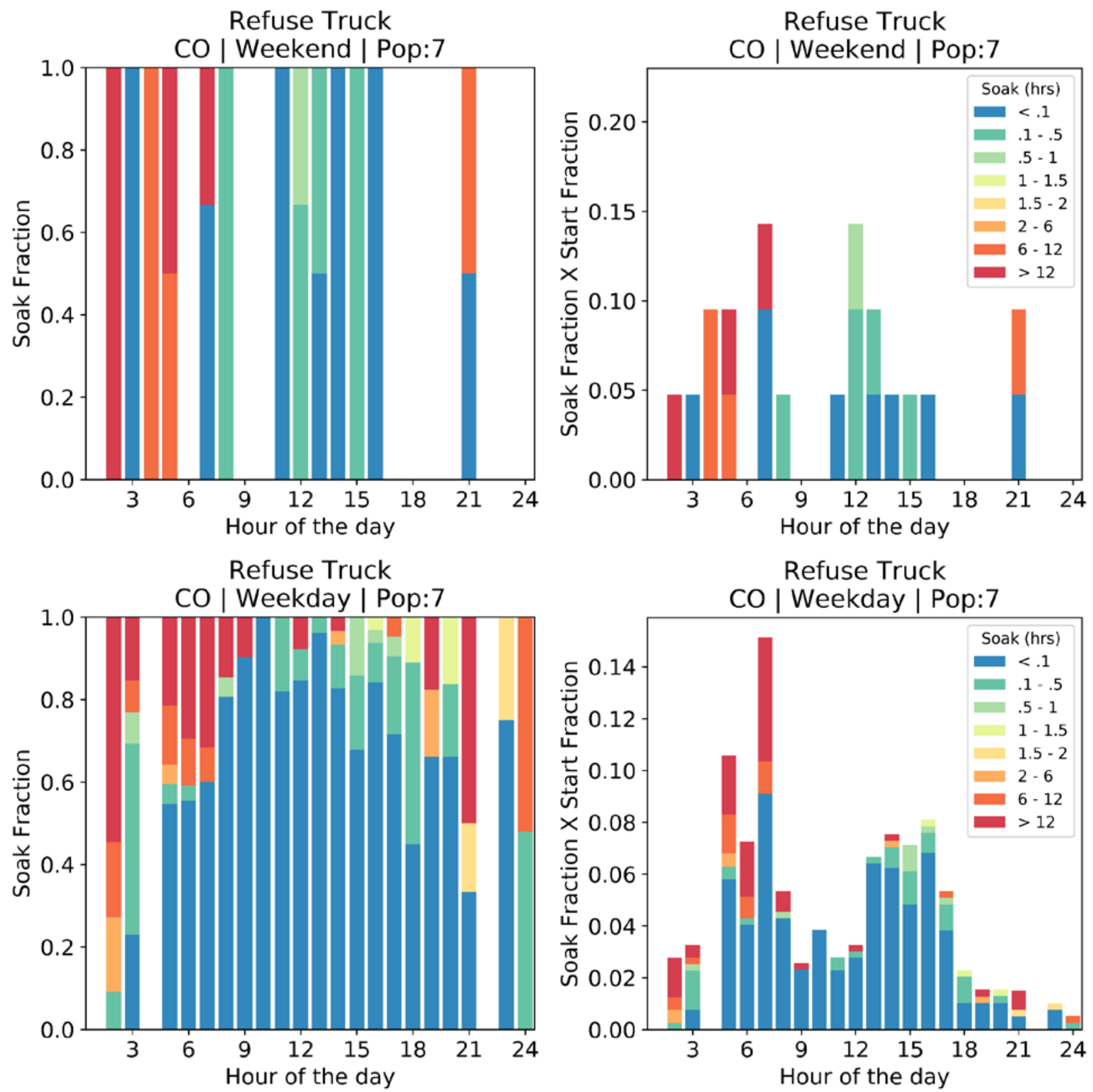

Figure H.8. Soak fraction and start fraction vs. hour of the day for Refuse Truck - CO 

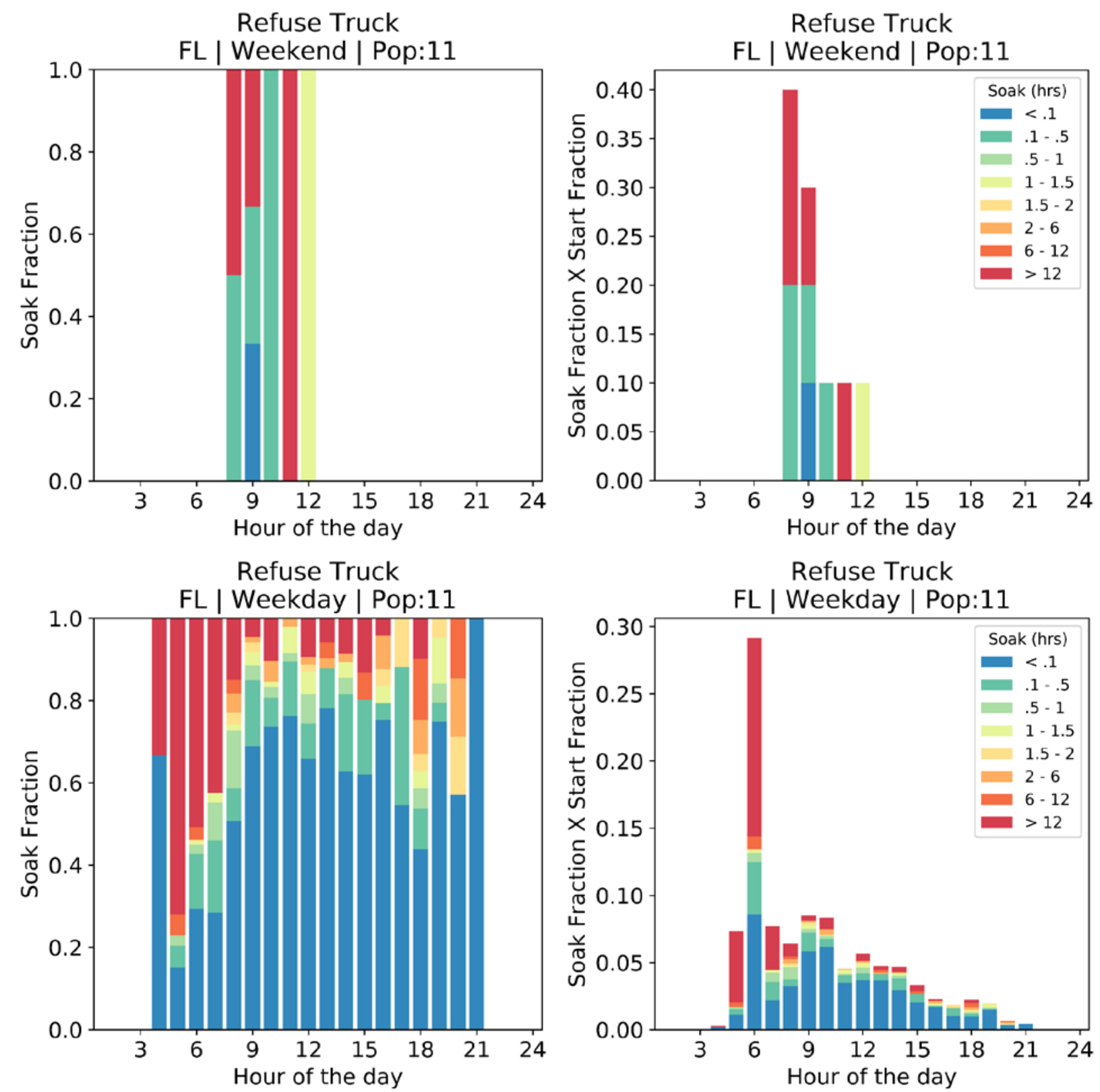

Figure H.9. Soak fraction and start fraction vs. hour of the day for Refuse Truck - FL 

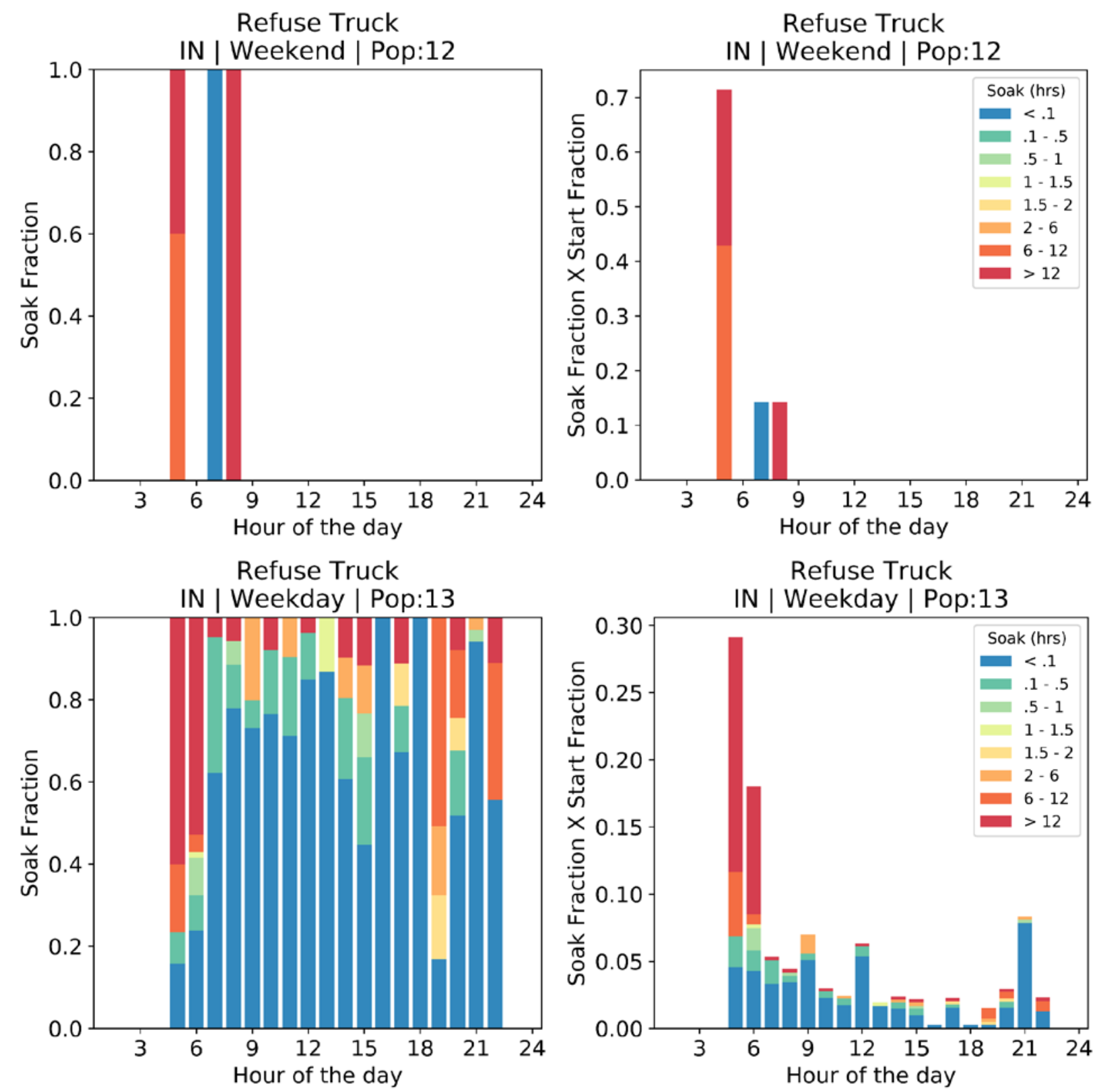

Figure H.10. Soak fraction and start fraction vs. hour of the day for Refuse Truck - IN 

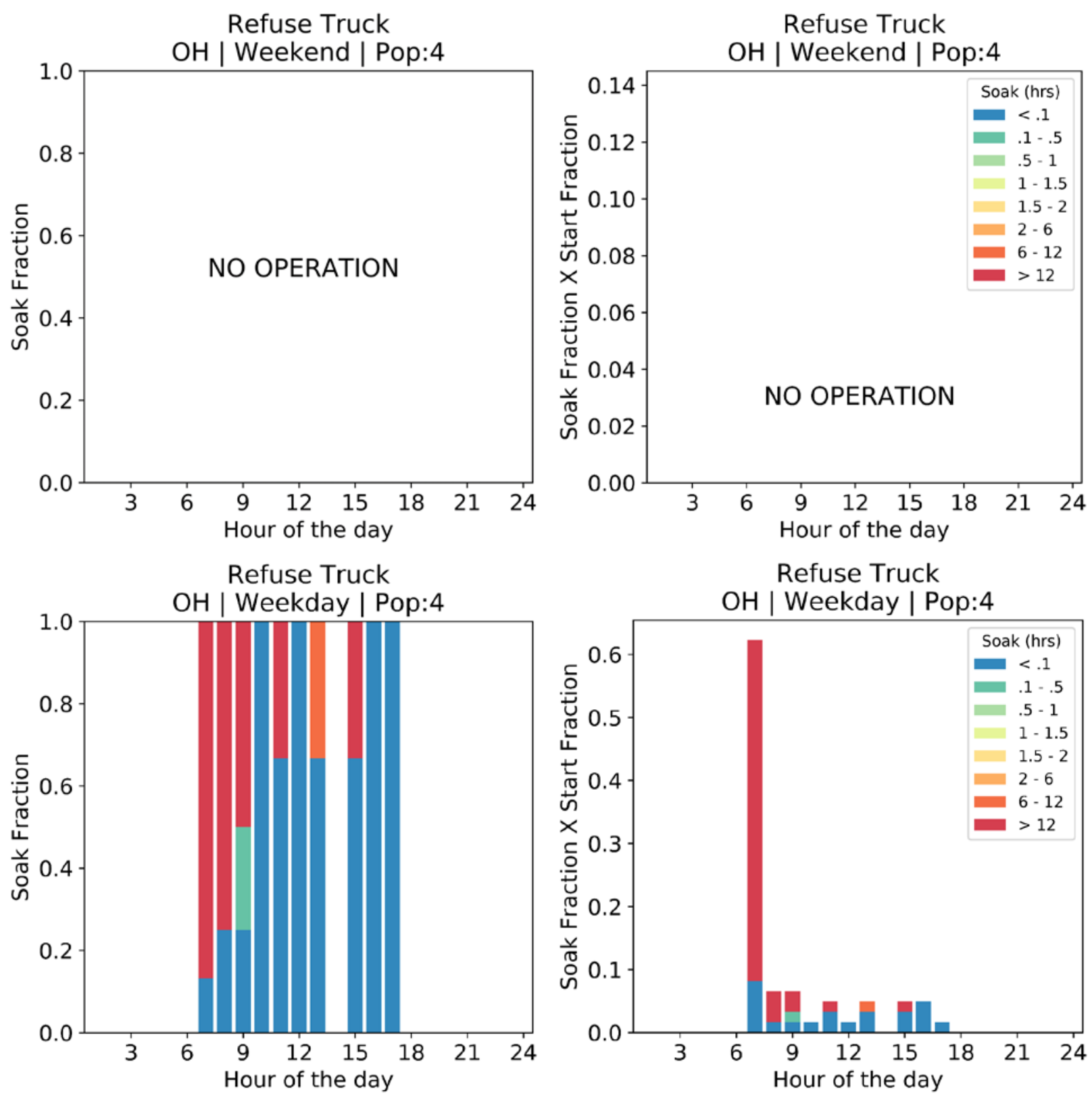

Figure H.11. Soak fraction and start fraction vs. hour of the day for Refuse Truck - OH 

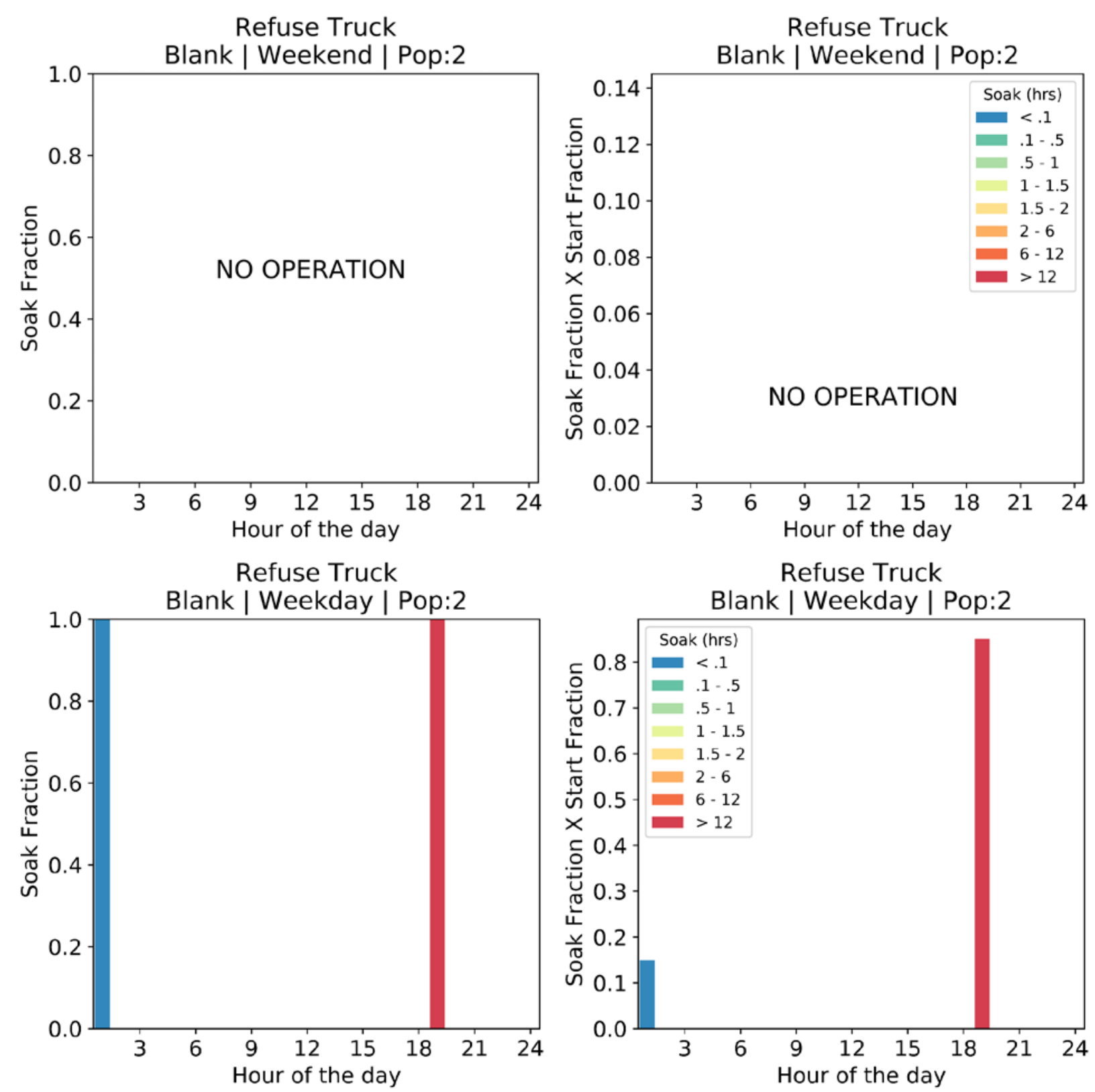

Figure H.12. Soak fraction and start fraction vs. hour of the day for Refuse Truck - Blank 


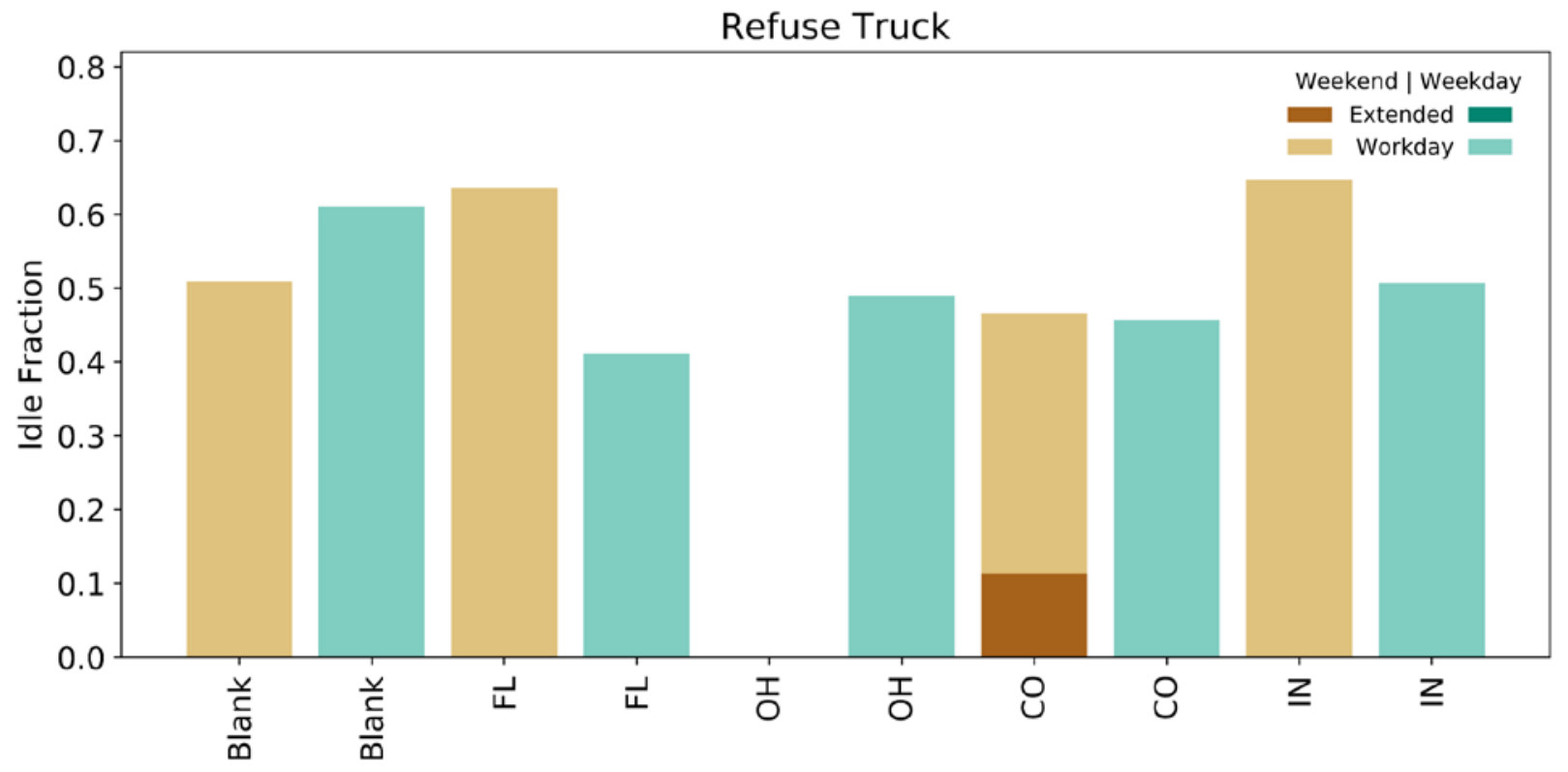

Figure H.13. Extended and workday idle fractions vs. day type and state for Refuse Truck 


\section{Single-Unit Short-Haul}

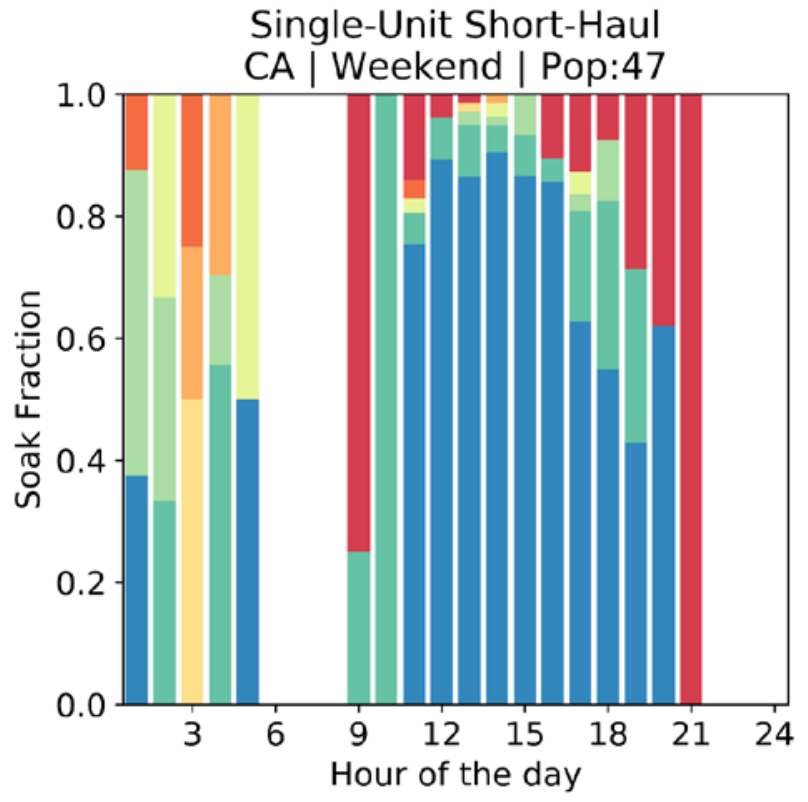

Single-Unit Short-Haul

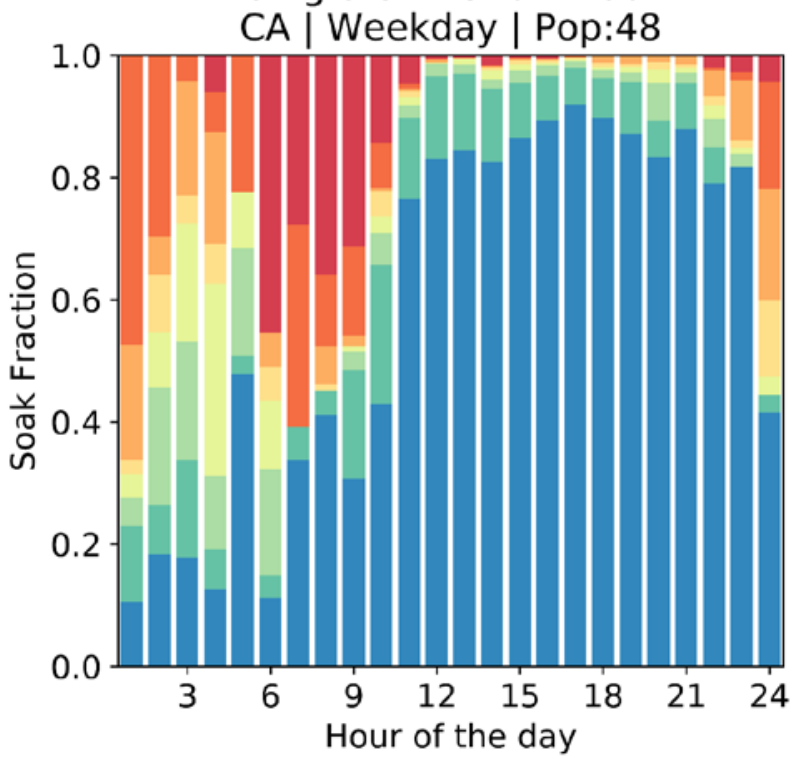

Single-Unit Short-Haul

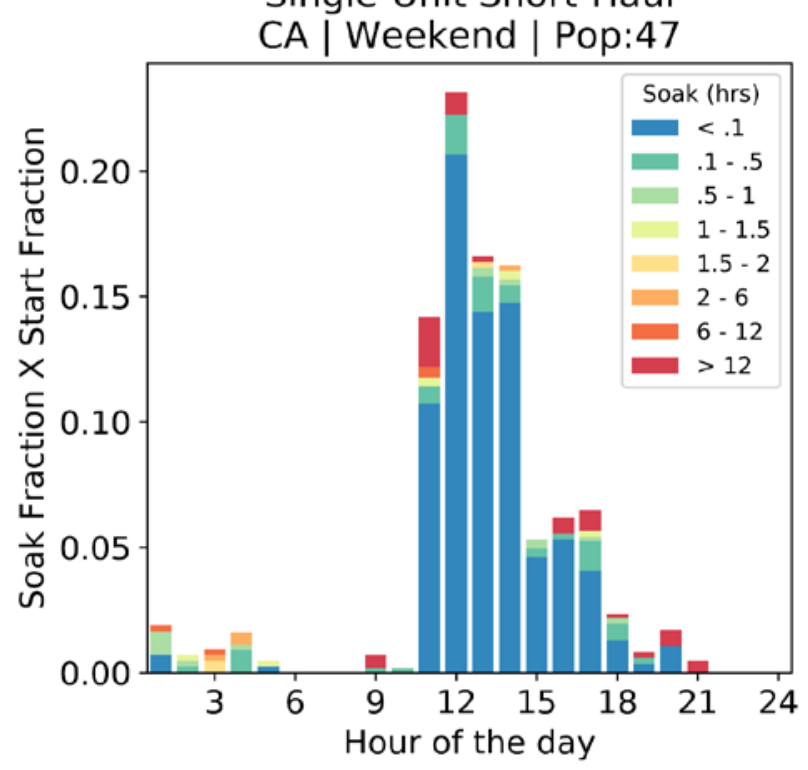

Single-Unit Short-Haul

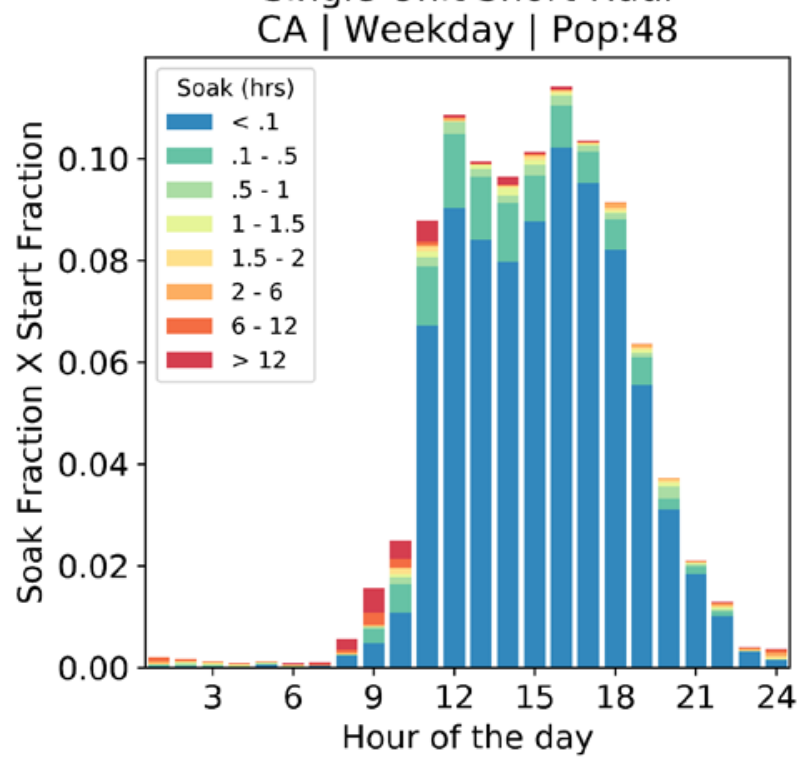

Figure H.14. Soak fraction and start fraction vs. hour of the day for Single-Unit Short-Haul - CA 


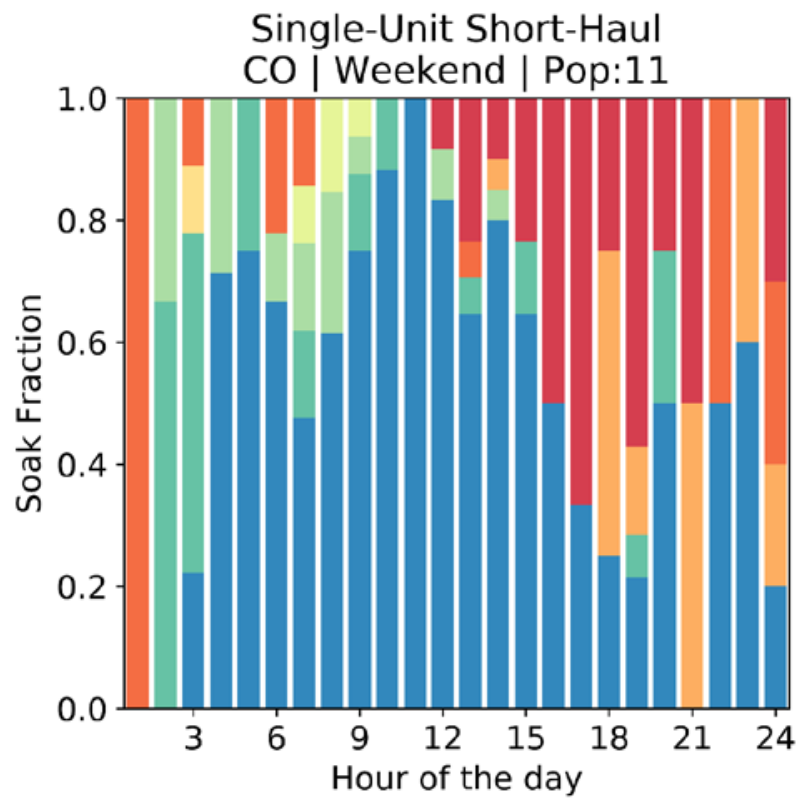

Single-Unit Short-Haul

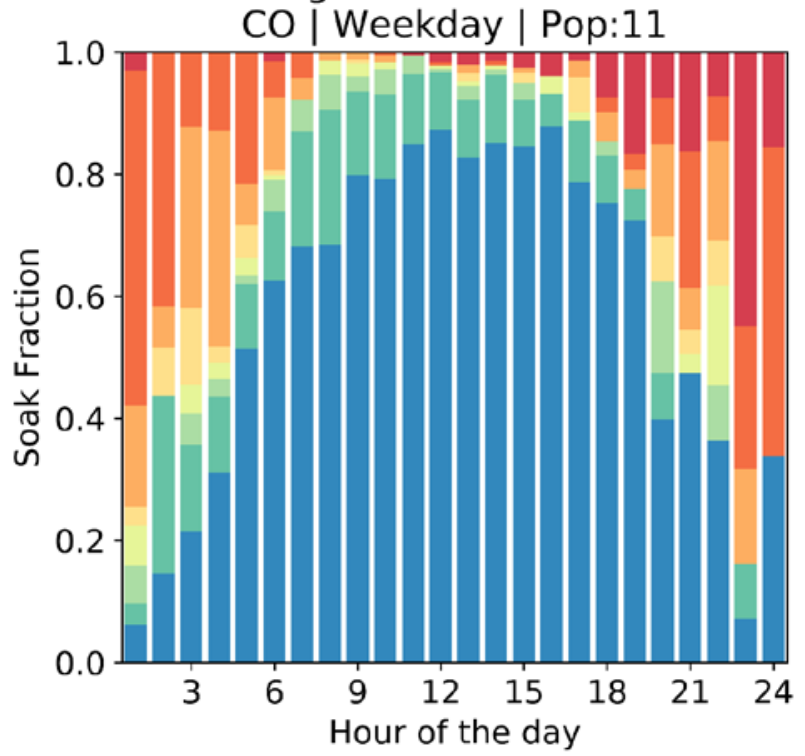

Single-Unit Short-Haul

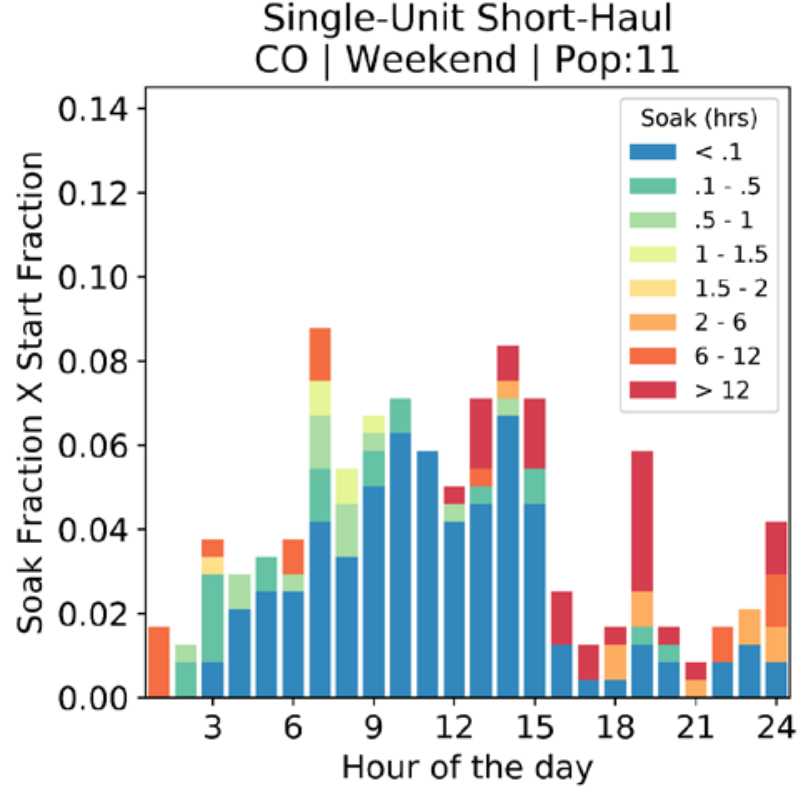

Single-Unit Short-Haul CO | Weekday | Pop:11

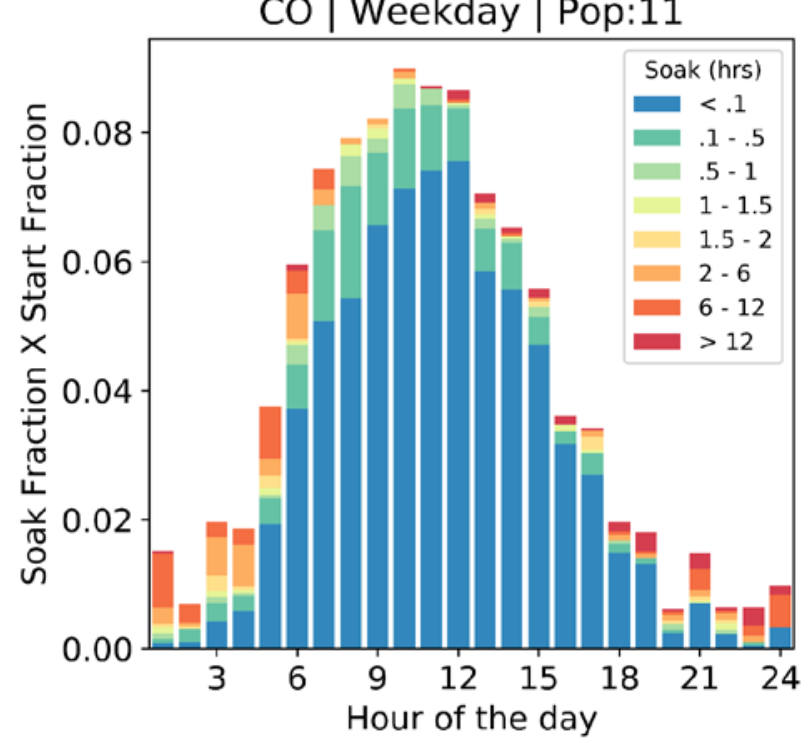

Figure H.15. Soak fraction and start fraction vs. hour of the day for Single-Unit Short-Haul - CO 

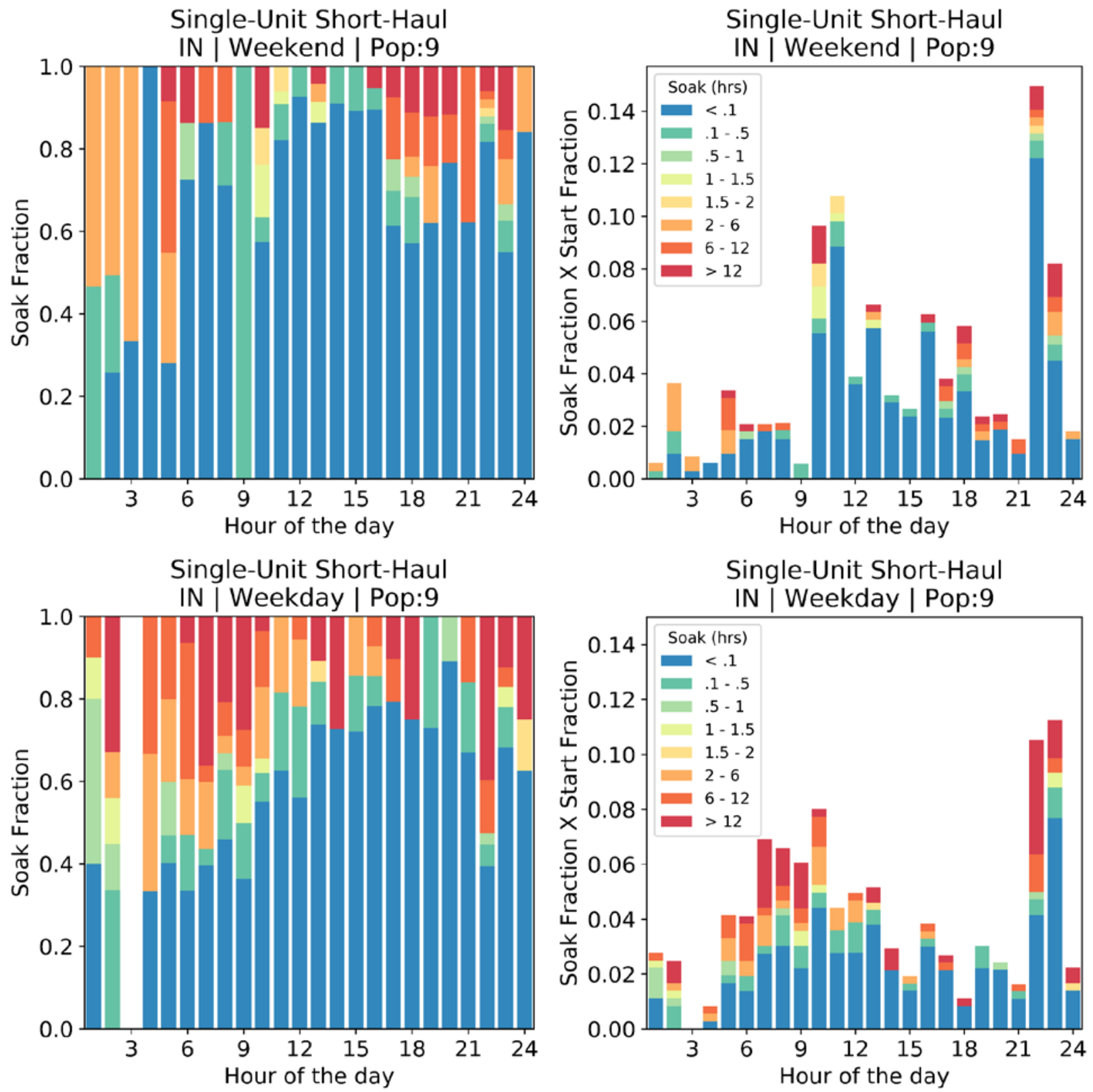

Figure H.16. Soak fraction and start fraction vs. hour of the day for Single-Unit Short-Haul - IN 

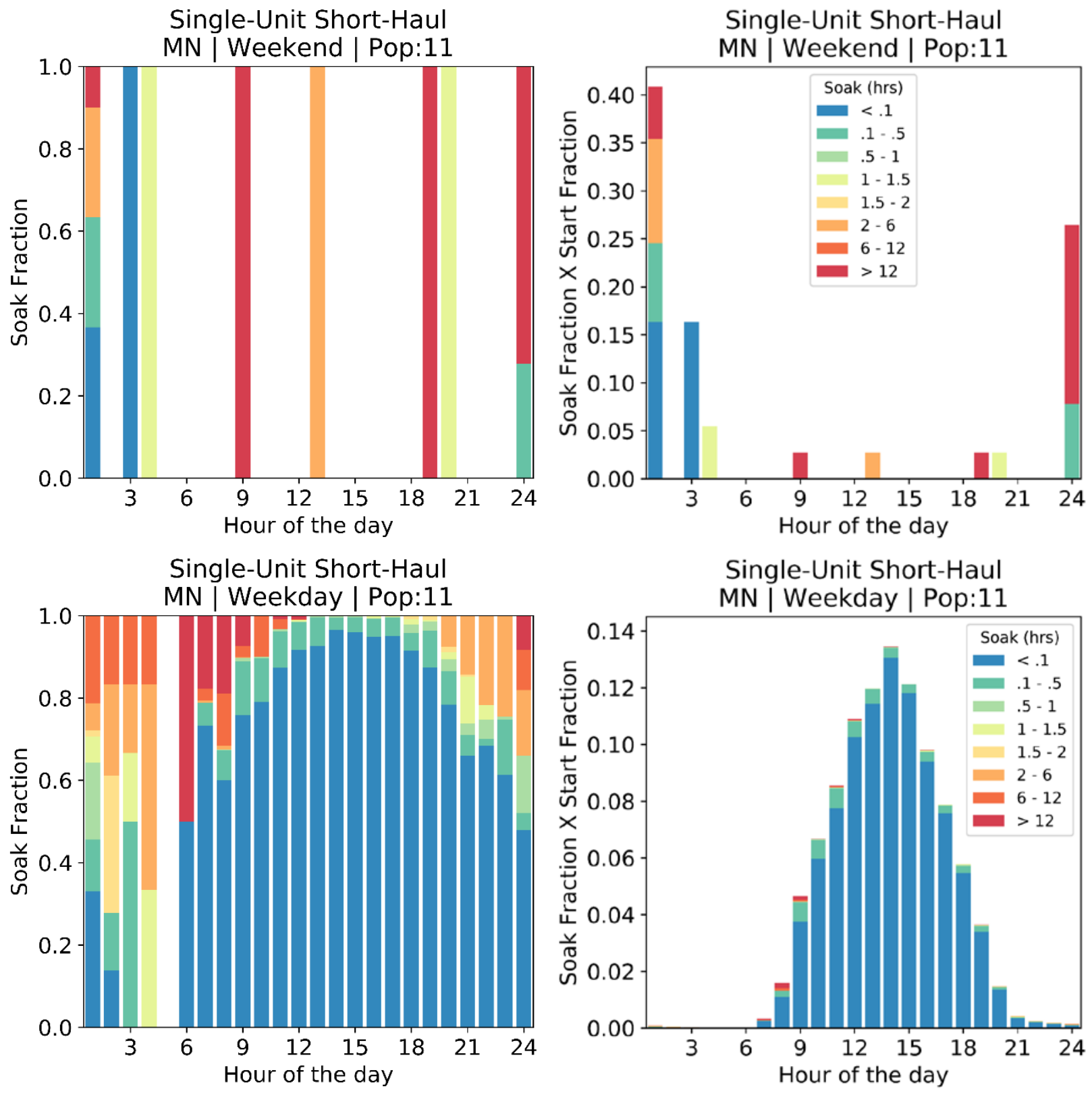

Figure H.17. Soak fraction and start fraction vs. hour of the day for Single-Unit Short-Haul - MN 


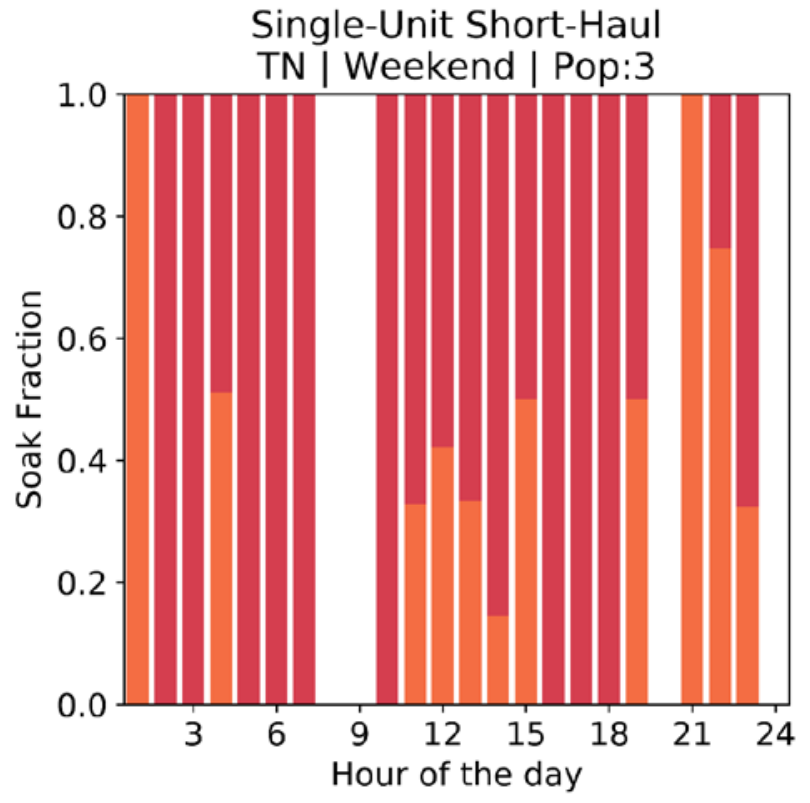

Single-Unit Short-Haul

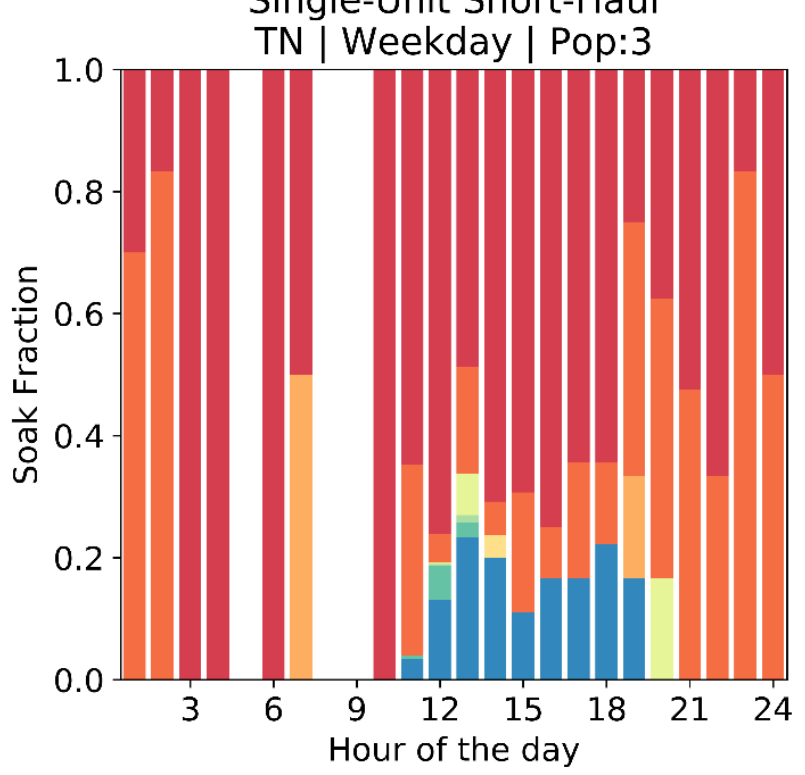

Single-Unit Short-Haul

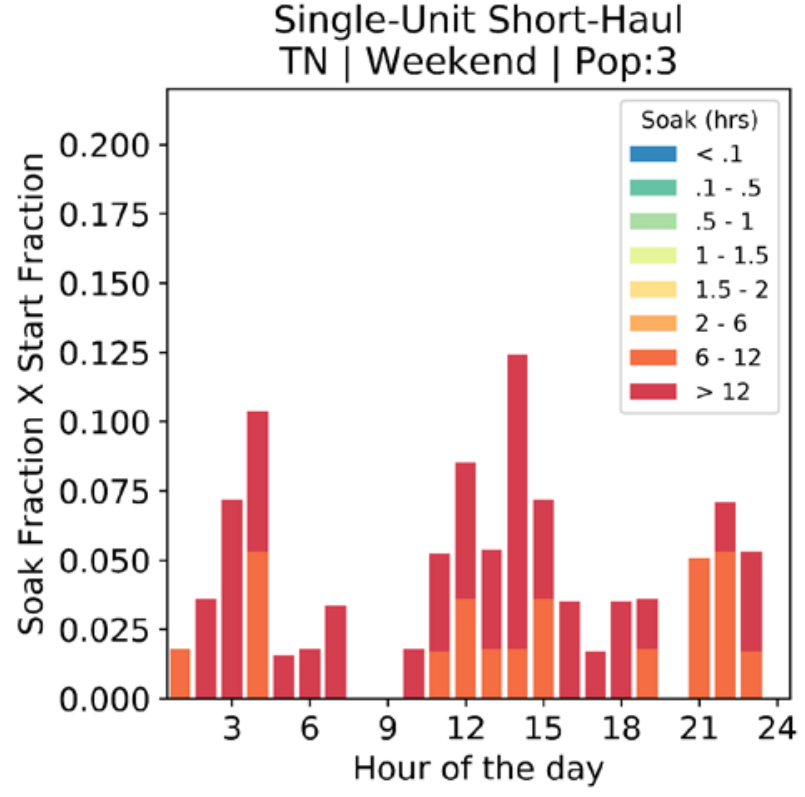

Single-Unit Short-Haul TN | Weekday | Pop:3

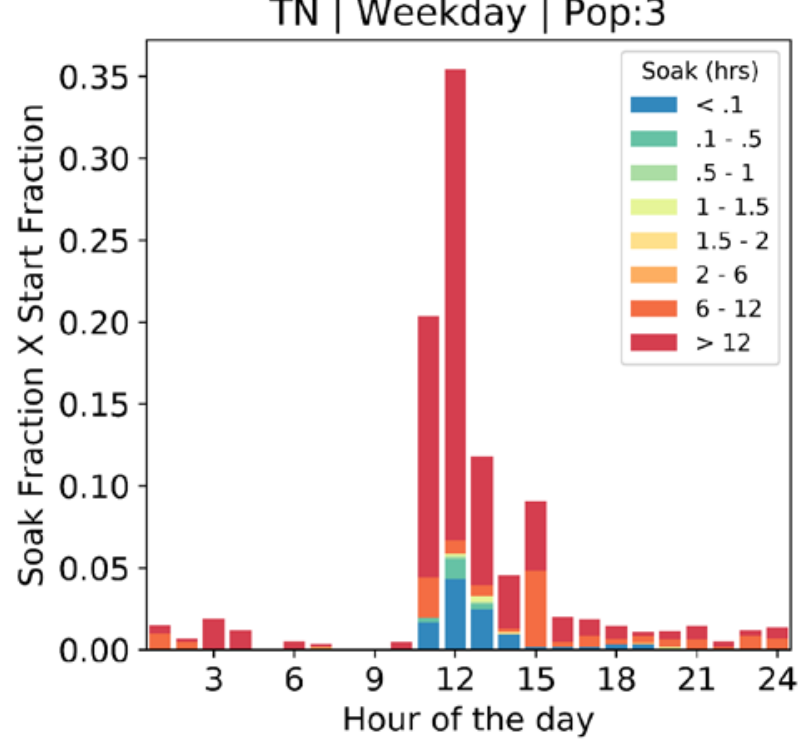

Figure H.18. Soak fraction and start fraction vs. hour of the day for Single-Unit Short-Haul - TN 

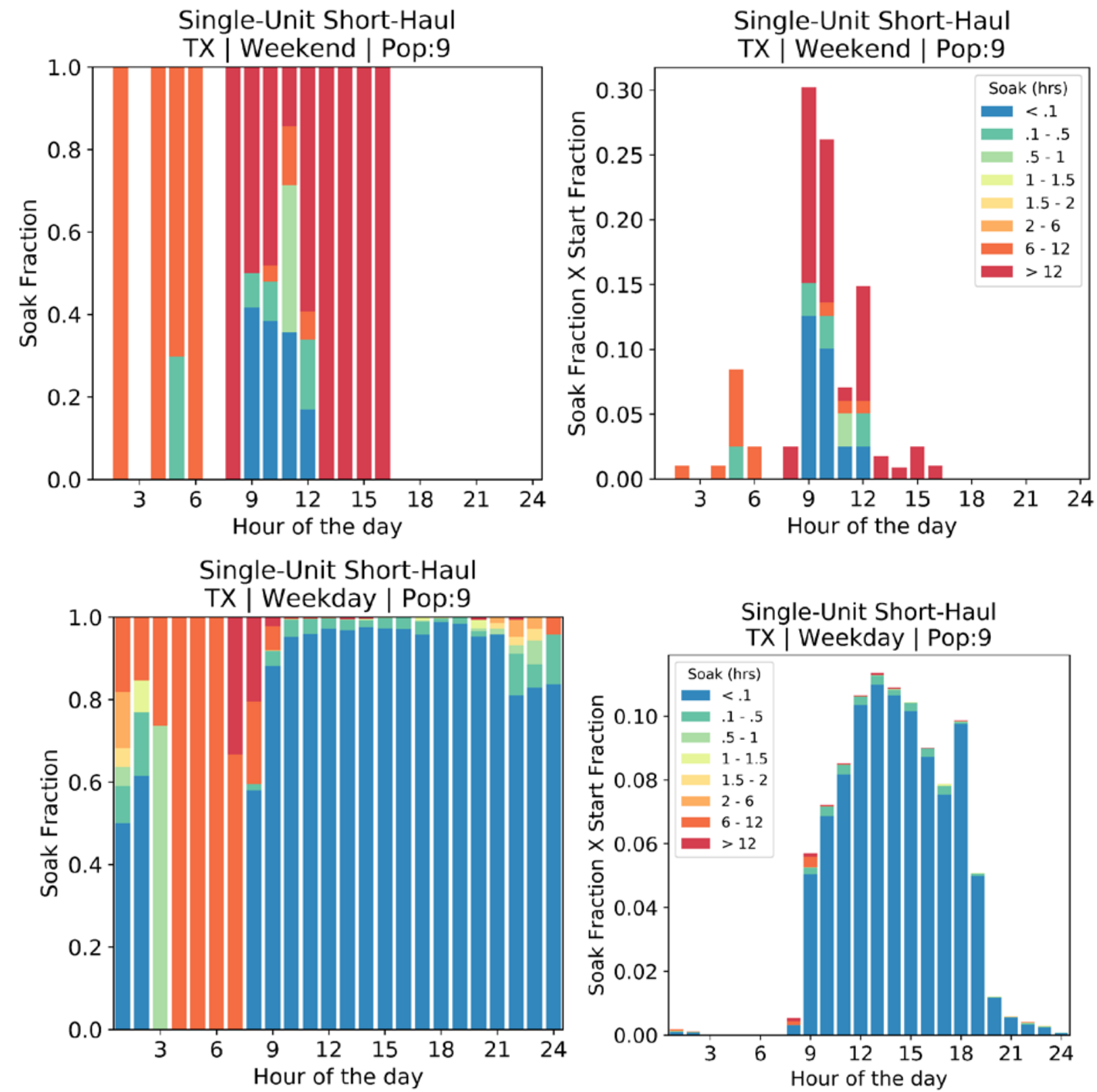

Figure H.19. Soak fraction and start fraction vs. hour of the day for Single-Unit Short-Haul - TX 

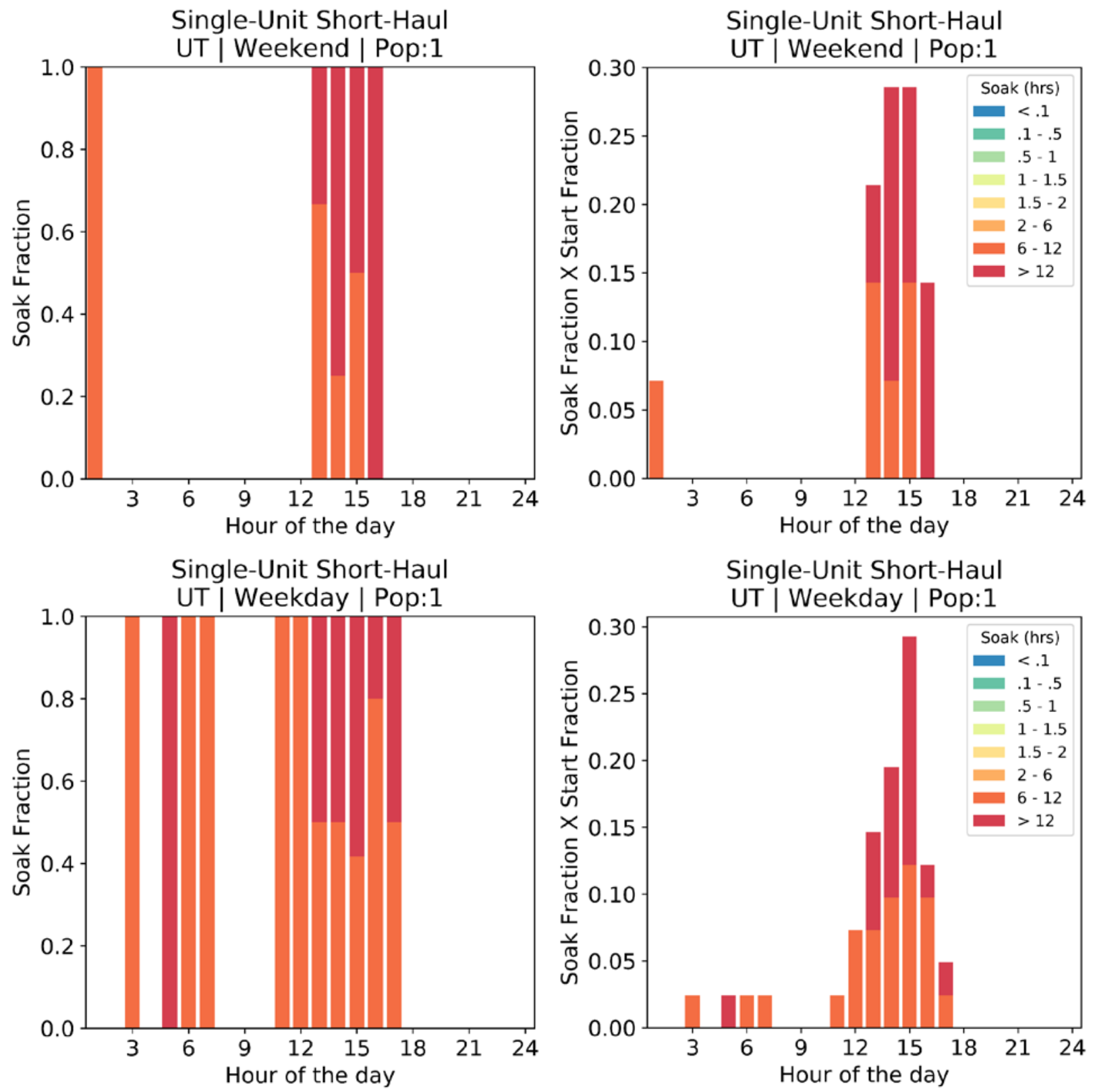

Figure H.20. Soak fraction and start fraction vs. hour of the day for Single-Unit Short-Haul - UT 

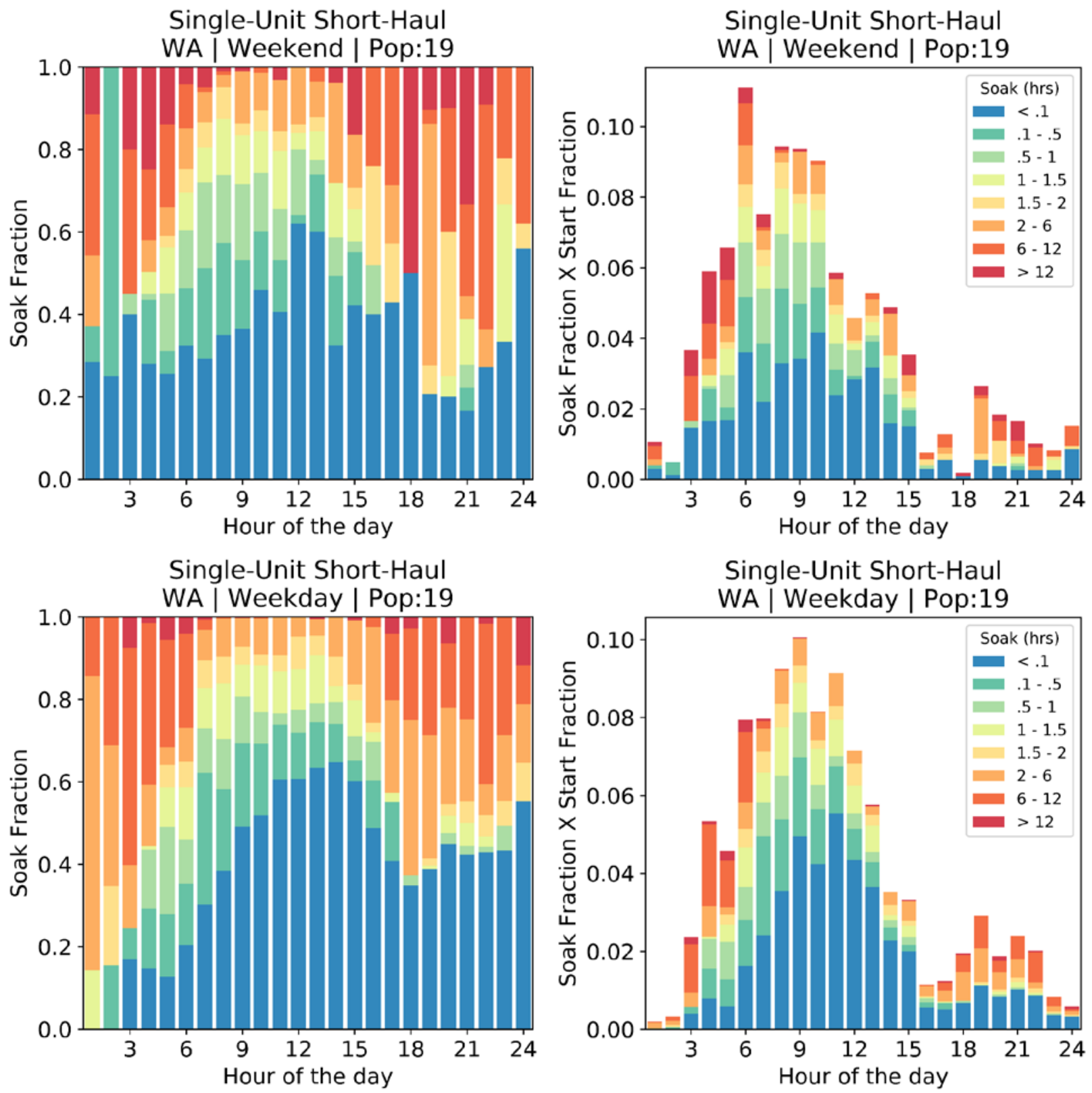

Figure H.21. Soak fraction and start fraction vs. hour of the day for Single-Unit Short-Haul - WA 

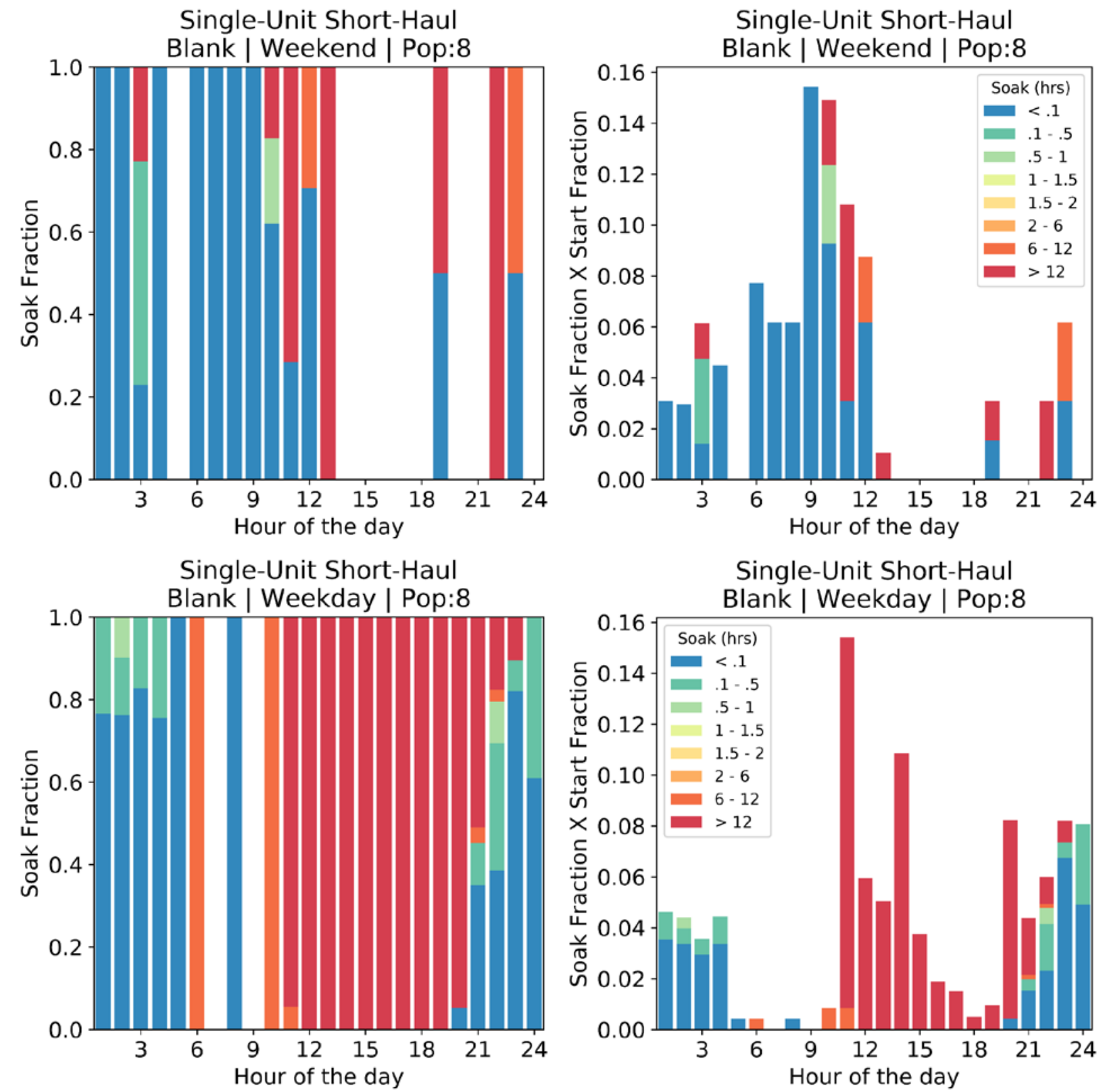

Figure H.22. Soak fraction and start fraction vs. hour of the day for Single-Unit Short-Haul - Blank 


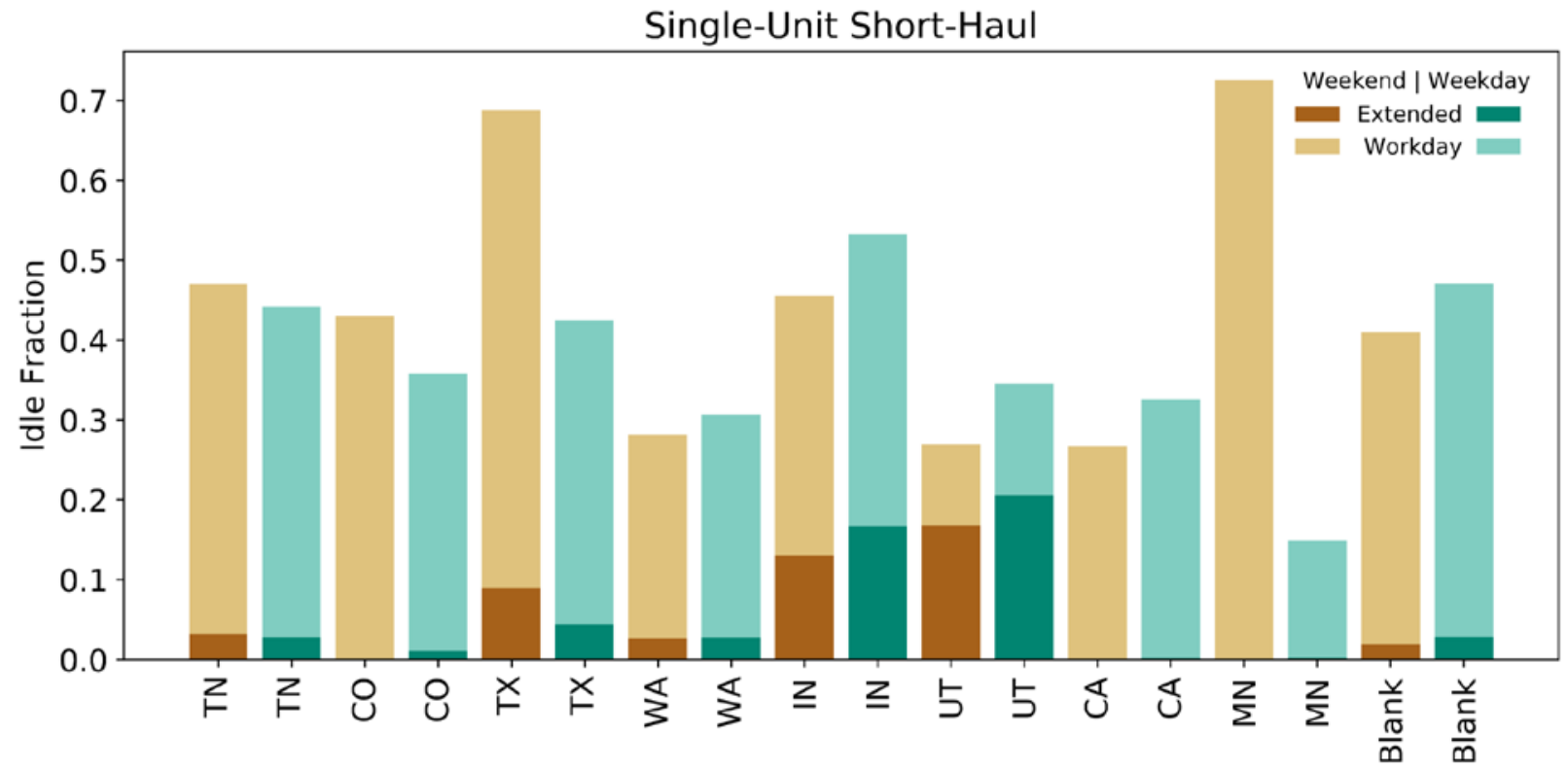

Figure H.23. Extended and workday idle fractions vs. day type and state for Single-Unit Short-Haul 
Combination-Unit Short-Haul
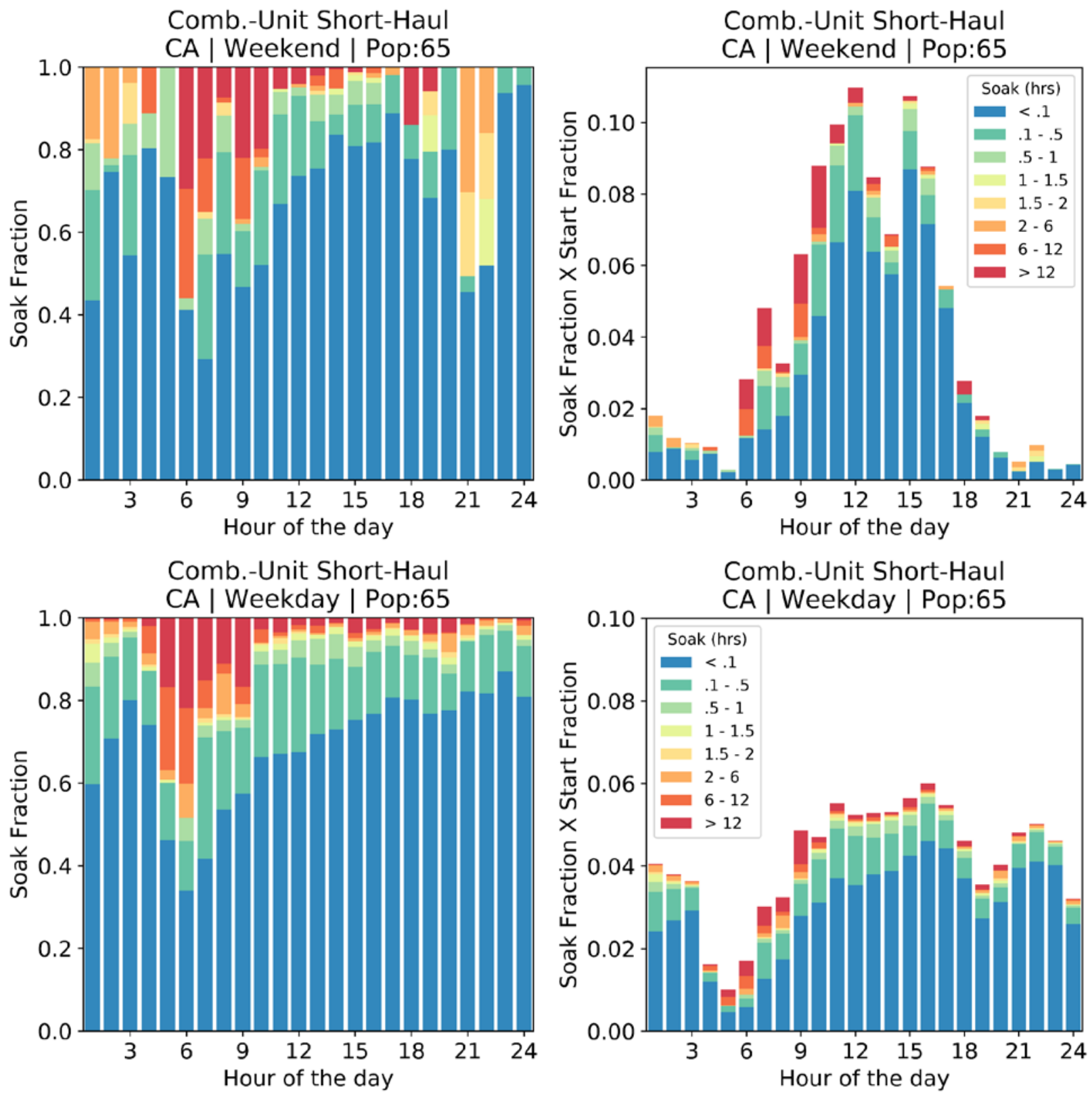

Figure H.24. Soak fraction and start fraction vs. hour of the day for Comb.-Unit Short-Haul - CA 

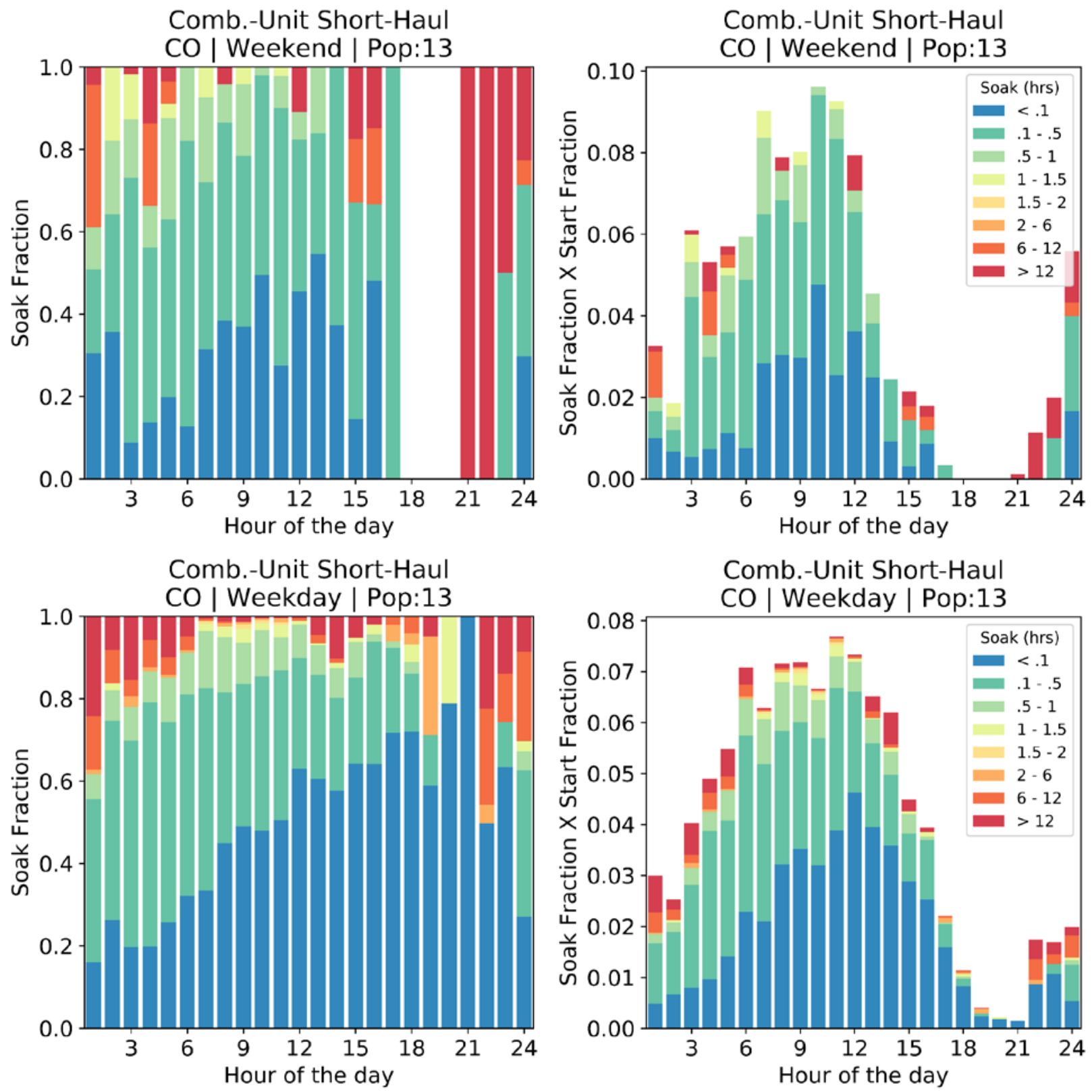

Figure H.25. Soak fraction and start fraction vs. hour of the day for Comb.-Unit Short-Haul - CO 

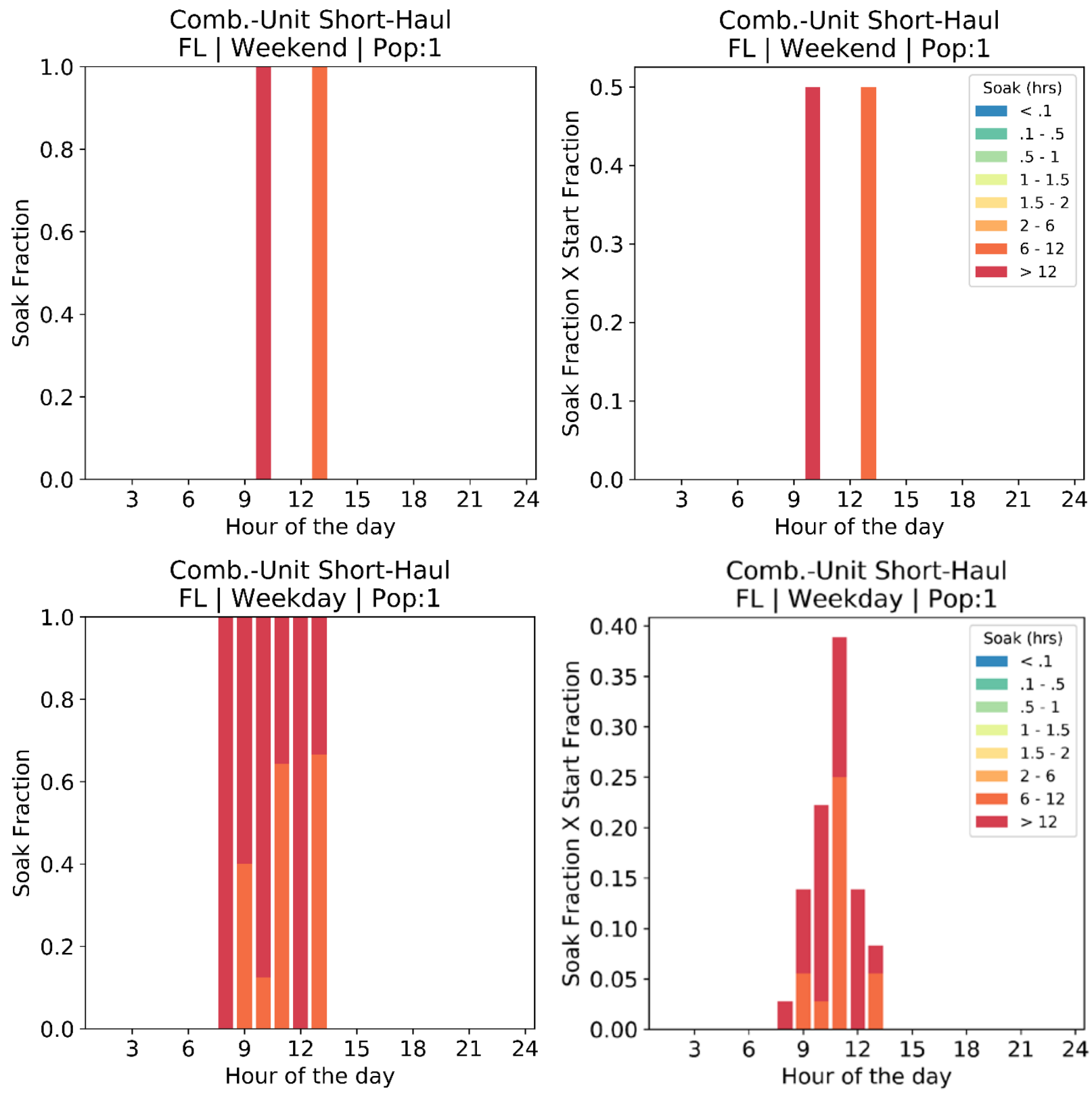

Figure H.26. Soak fraction and start fraction vs. hour of the day for Comb.-Unit Short-Haul - FL 

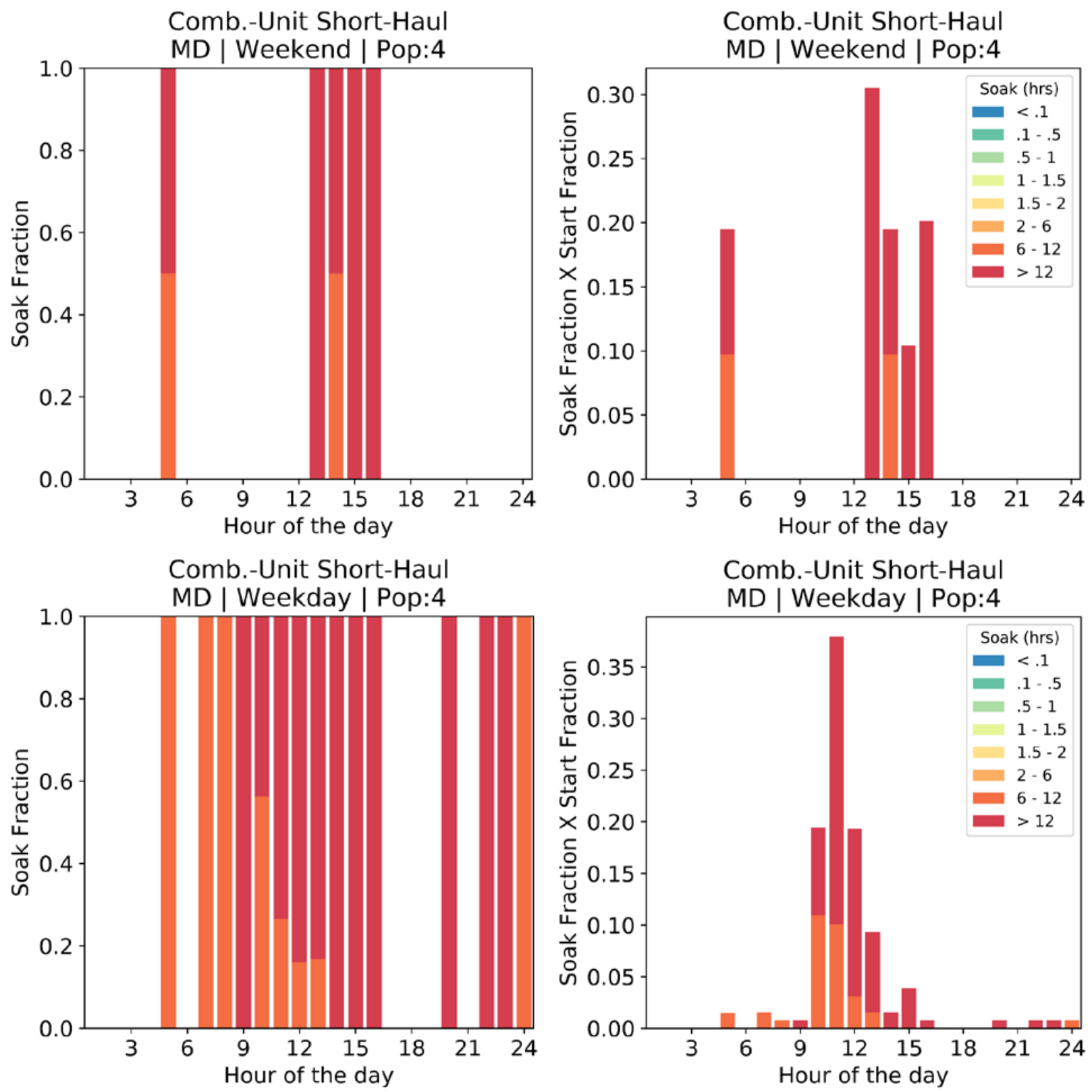

Figure H.27. Soak fraction and start fraction vs. hour of the day for Comb.-Unit Short-Haul - MD 

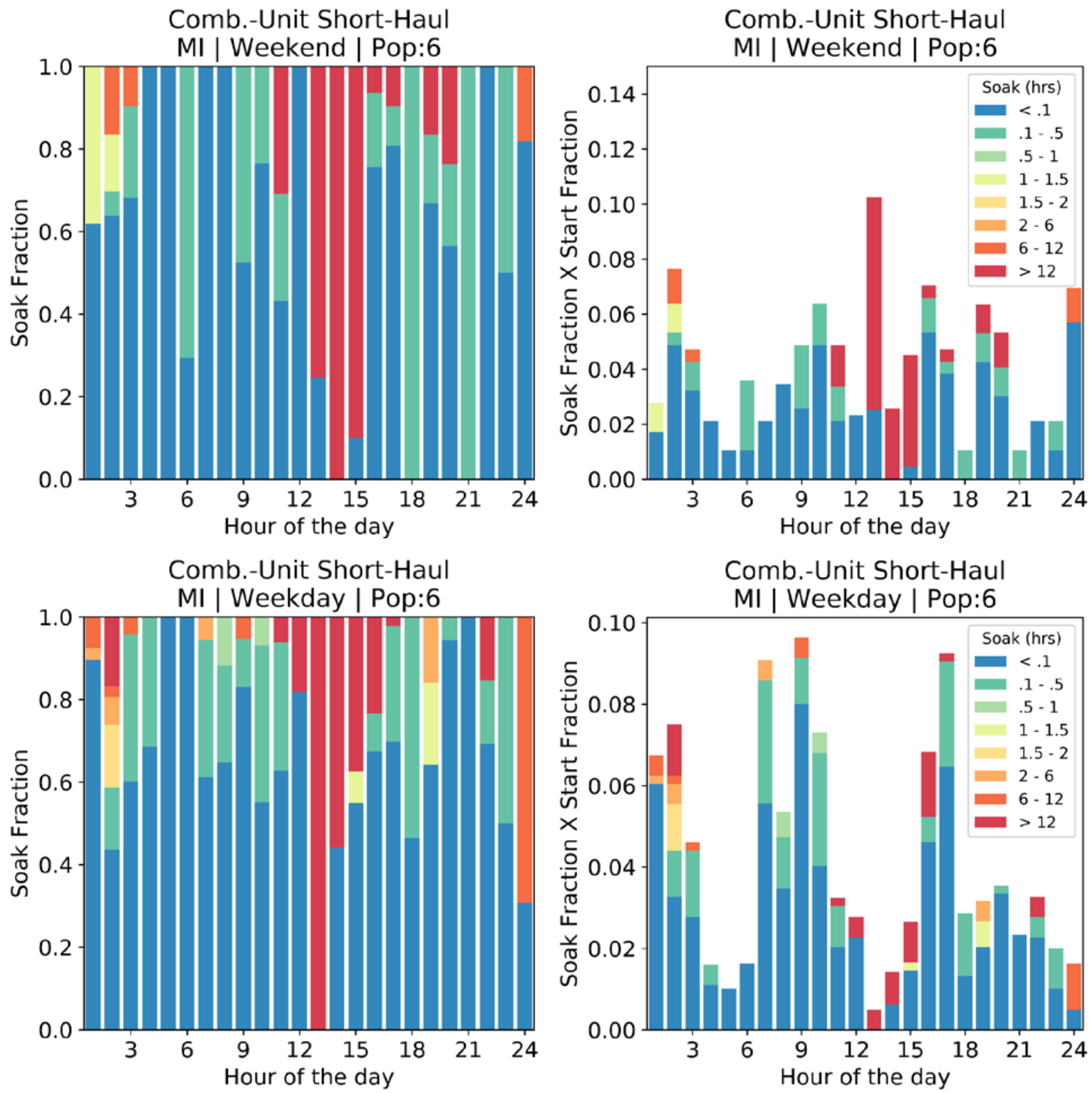

Figure H.28. Soak fraction and start fraction vs. hour of the day for Comb.-Unit Short-Haul - MI 

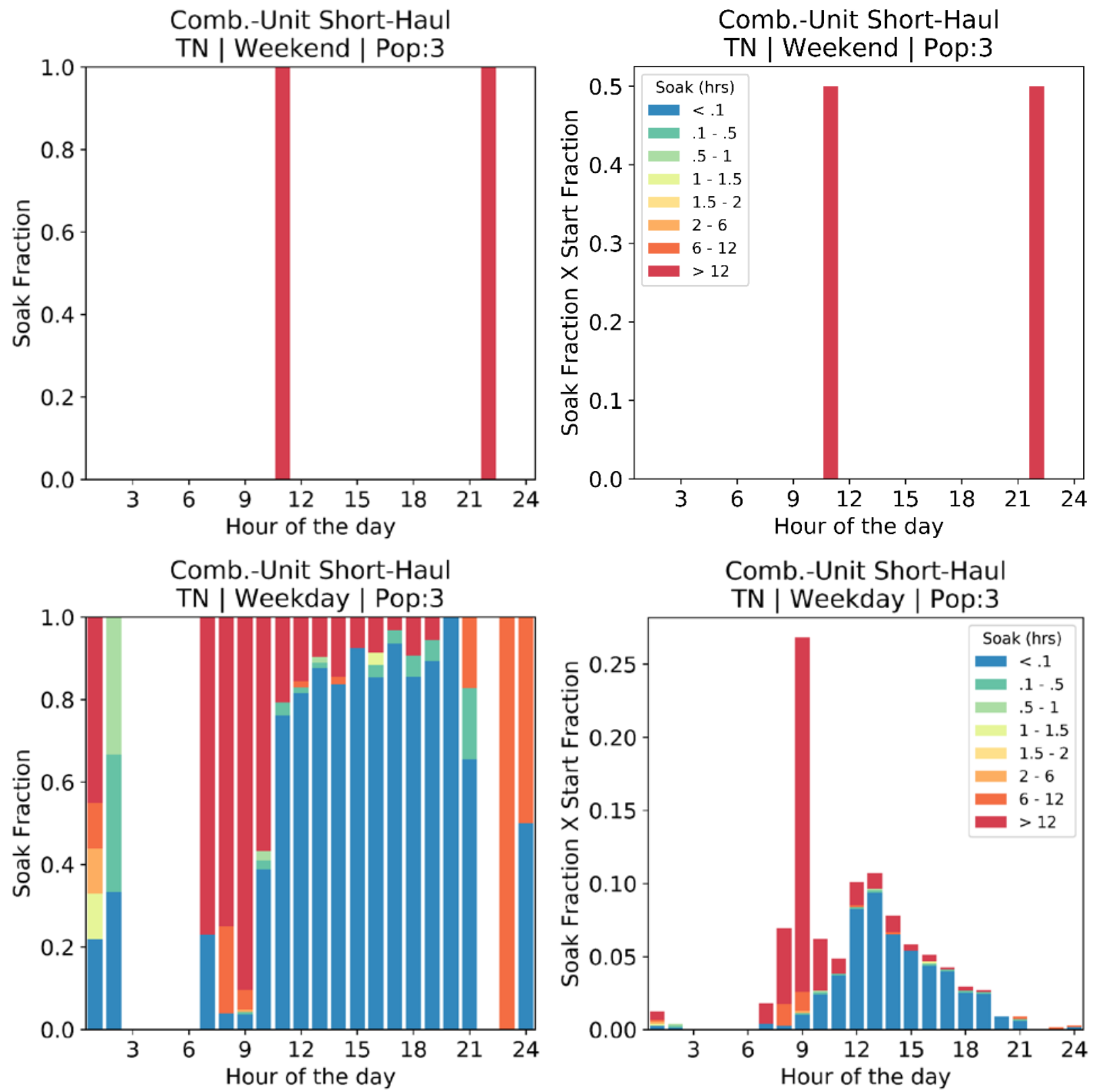

Figure H.29. Soak fraction and start fraction vs. hour of the day for Comb.-Unit Short-Haul - TN 

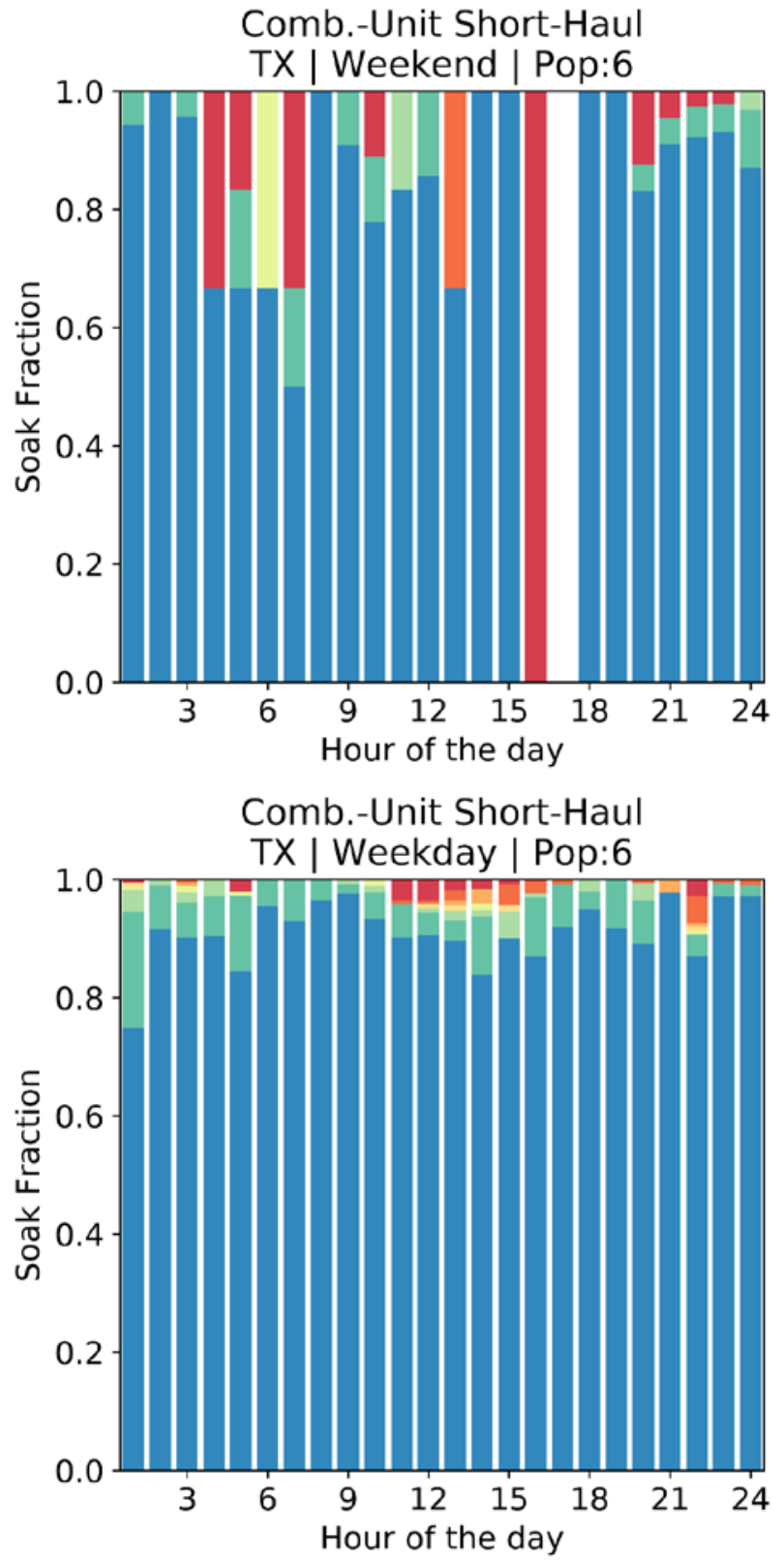

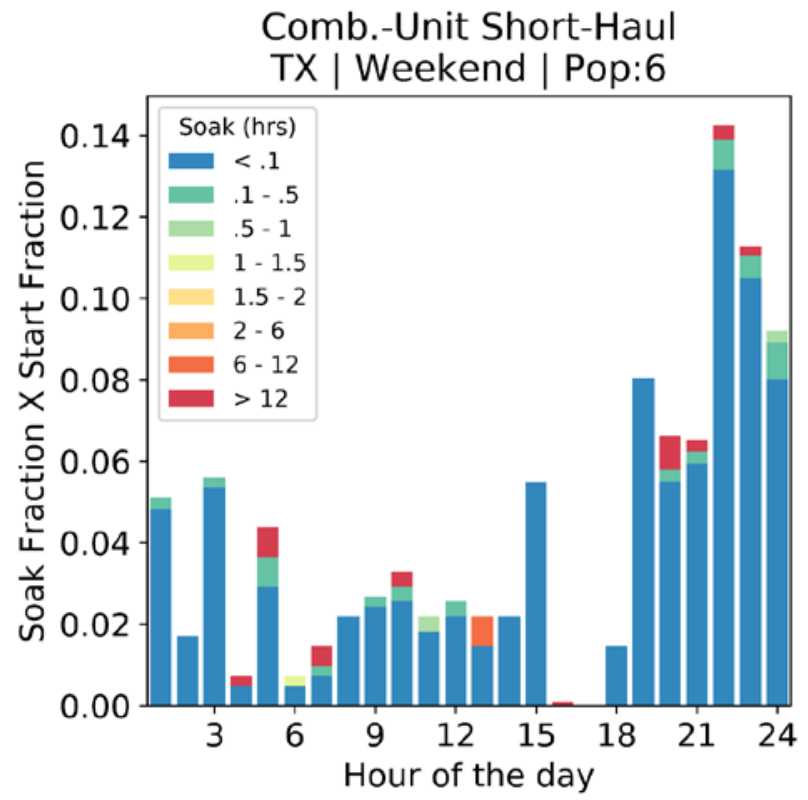

Comb.-Unit Short-Haul

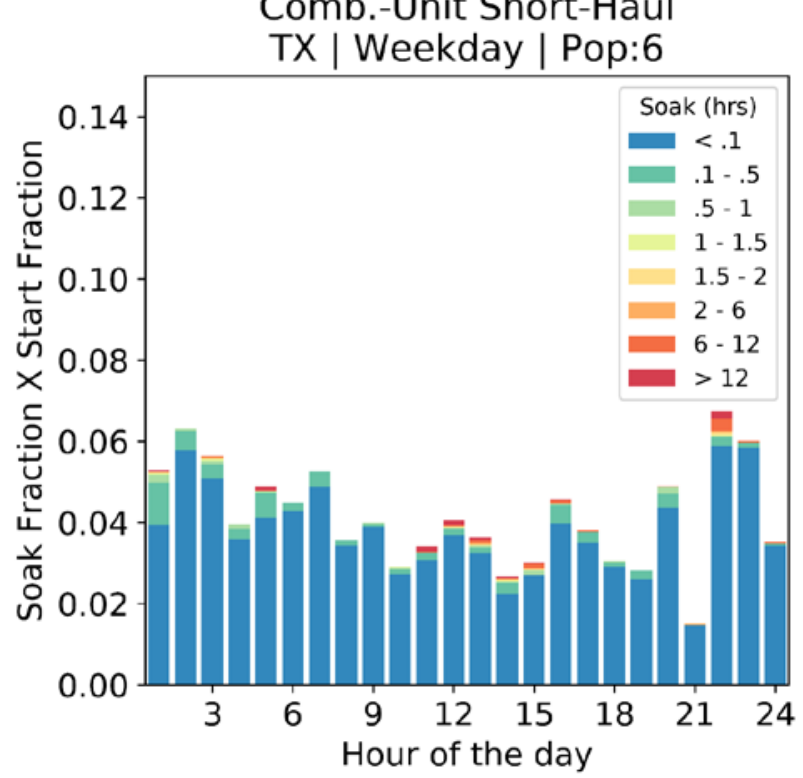

Figure H.30. Soak fraction and start fraction vs. hour of the day for Comb.-Unit Short-Haul - TX 

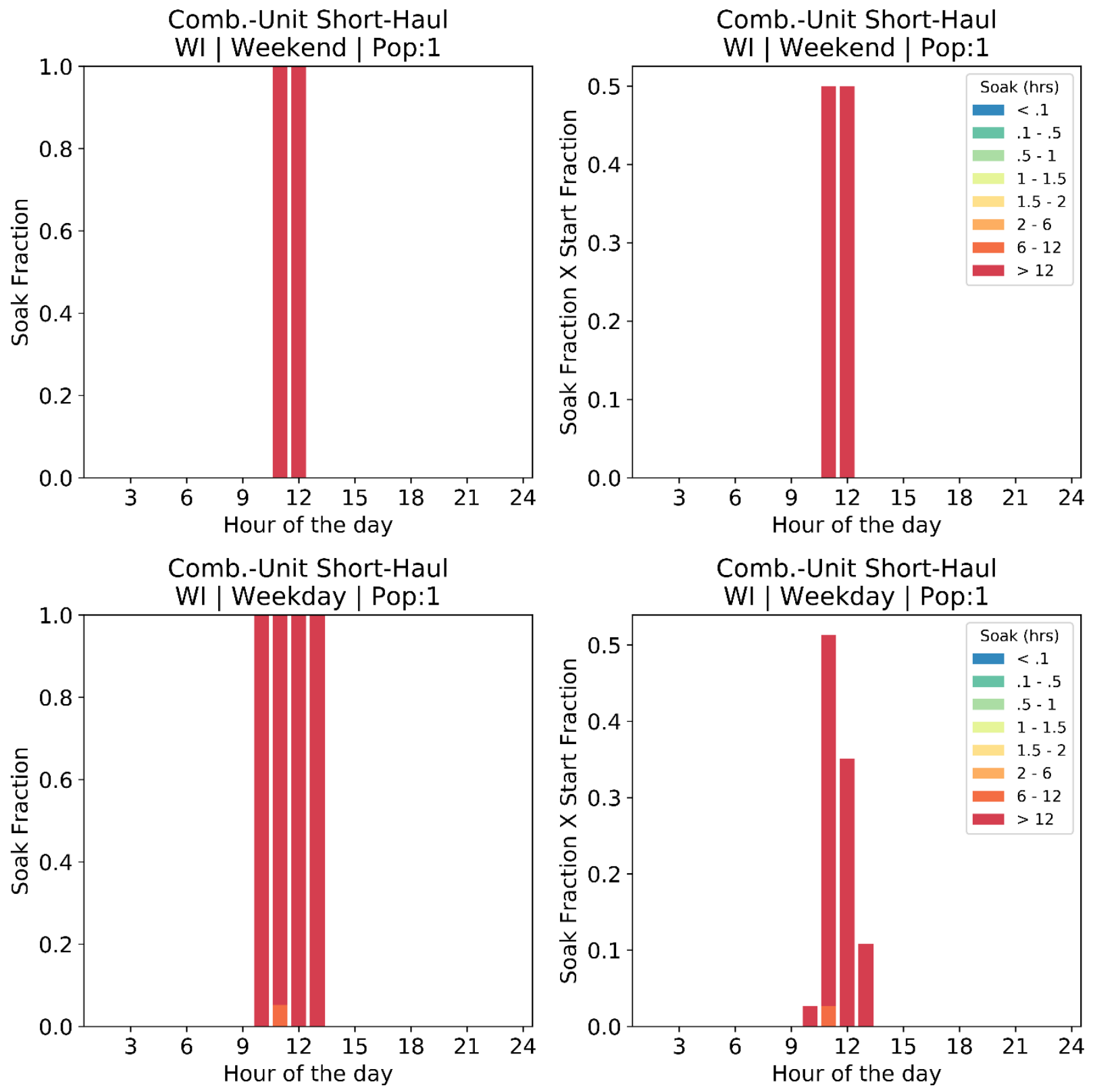

Figure H.31. Soak fraction and start fraction vs. hour of the day for Comb.-Unit Short-Haul - WI 

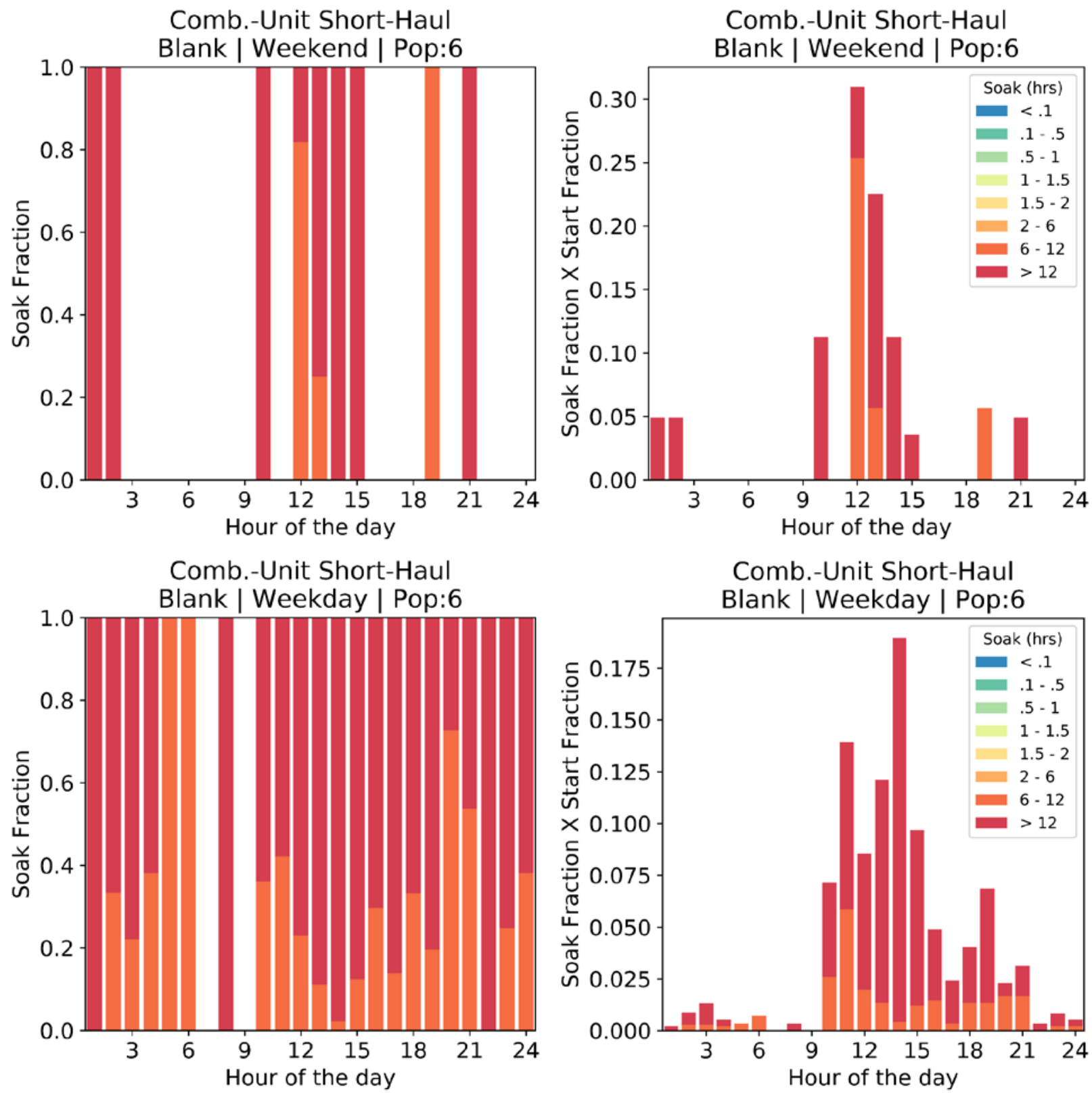

Figure H.32. Soak fraction and start fraction vs. hour of the day for Comb.-Unit Short-Haul - Blank 


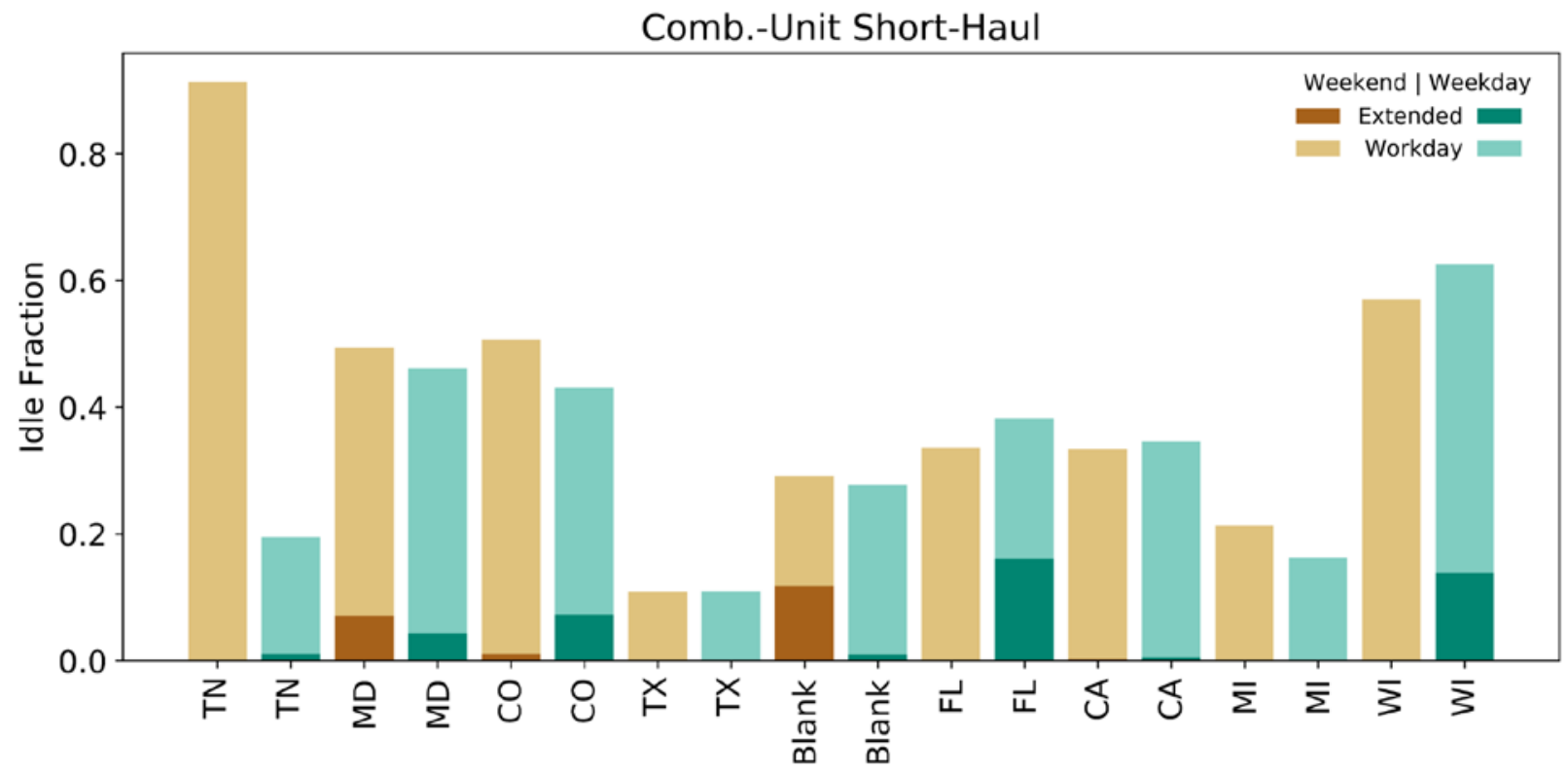

Figure H.33. Extended and workday idle fractions vs. day type and state for Comb.-Unit Short-Haul 


\section{Combination-Unit Long-Haul}
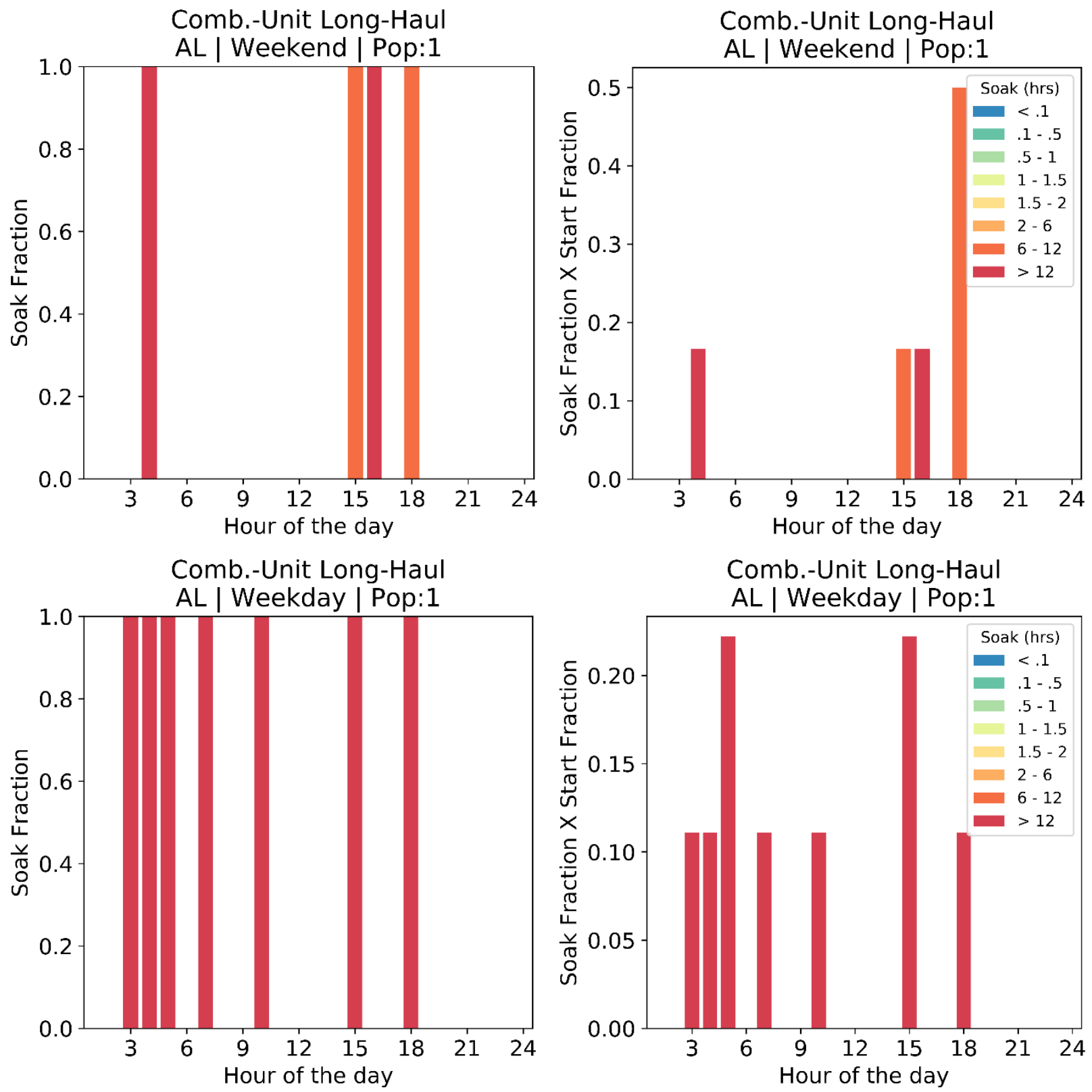

Figure H.34. Soak fraction and start fraction vs. hour of the day for Comb.-Unit Long-Haul - AL 

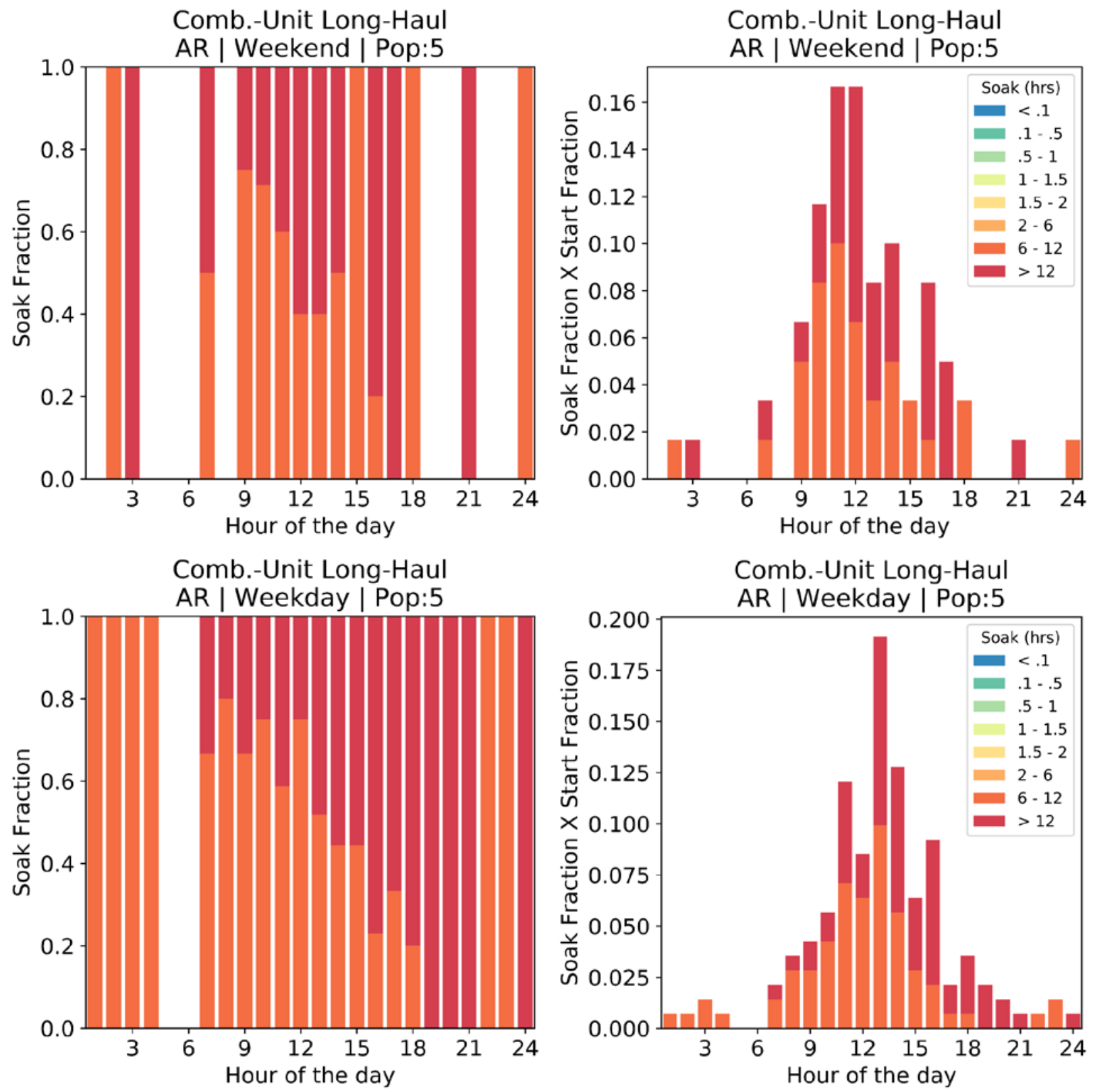

Figure H.35. Soak fraction and start fraction vs. hour of the day for Comb.-Unit Long-Haul - AR 

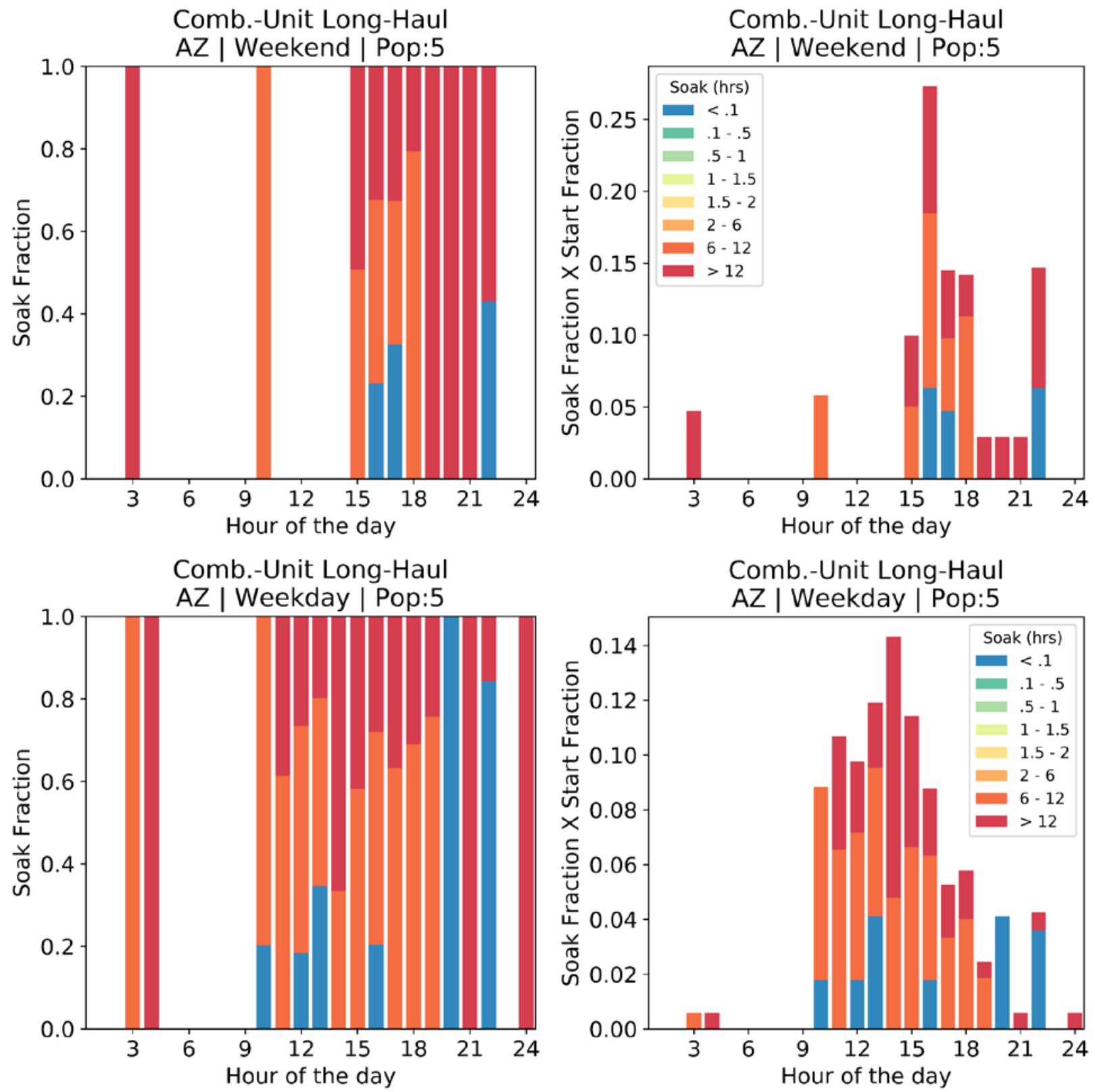

Figure H.36. Soak fraction and start fraction vs. hour of the day for Comb.-Unit Long-Haul - AZ 

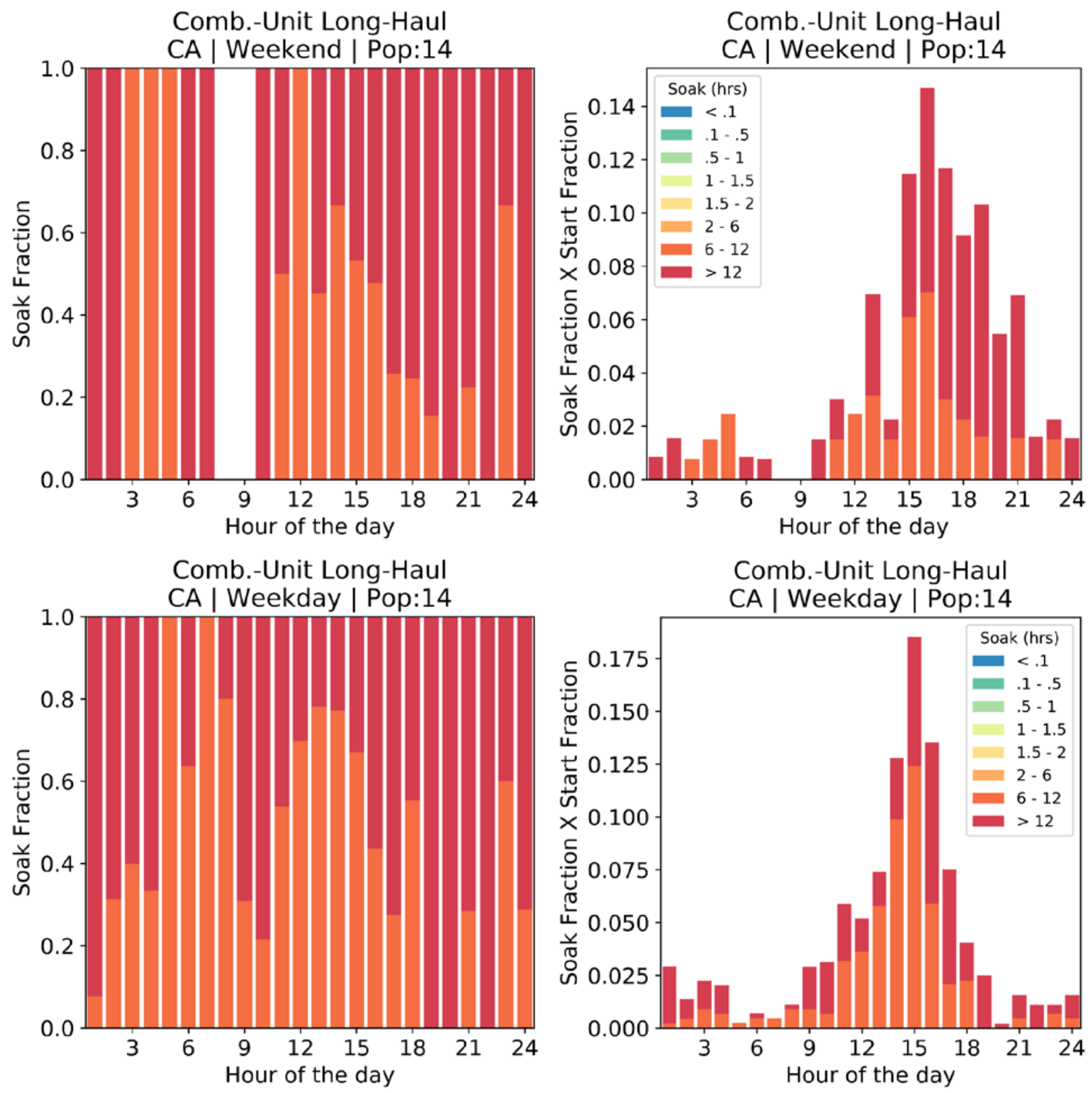

Figure H.37. Soak fraction and start fraction vs. hour of the day for Comb.-Unit Long-Haul - CA 

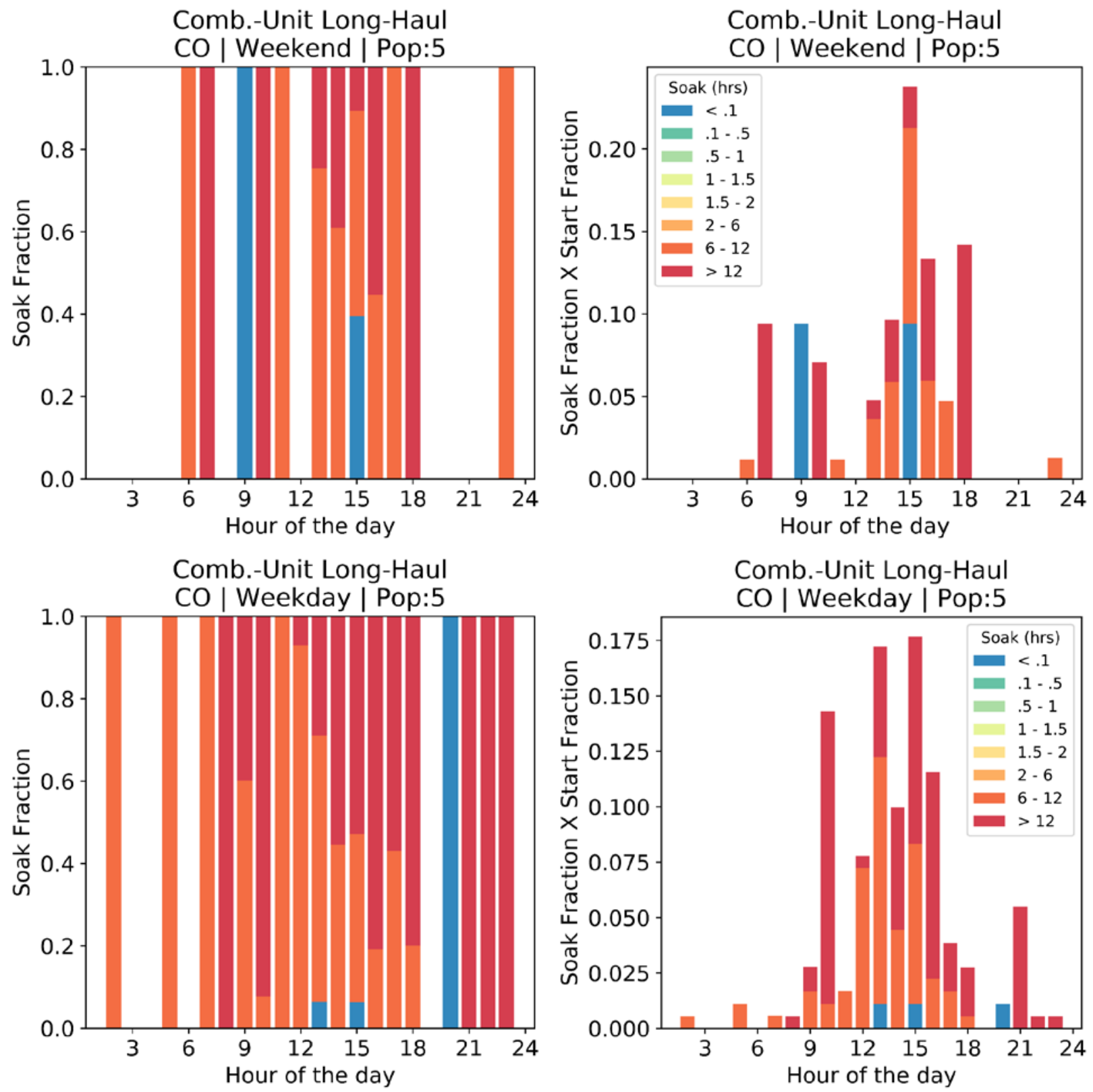

Figure H.38. Soak fraction and start fraction vs. hour of the day for Comb.-Unit Long-Haul - CO 

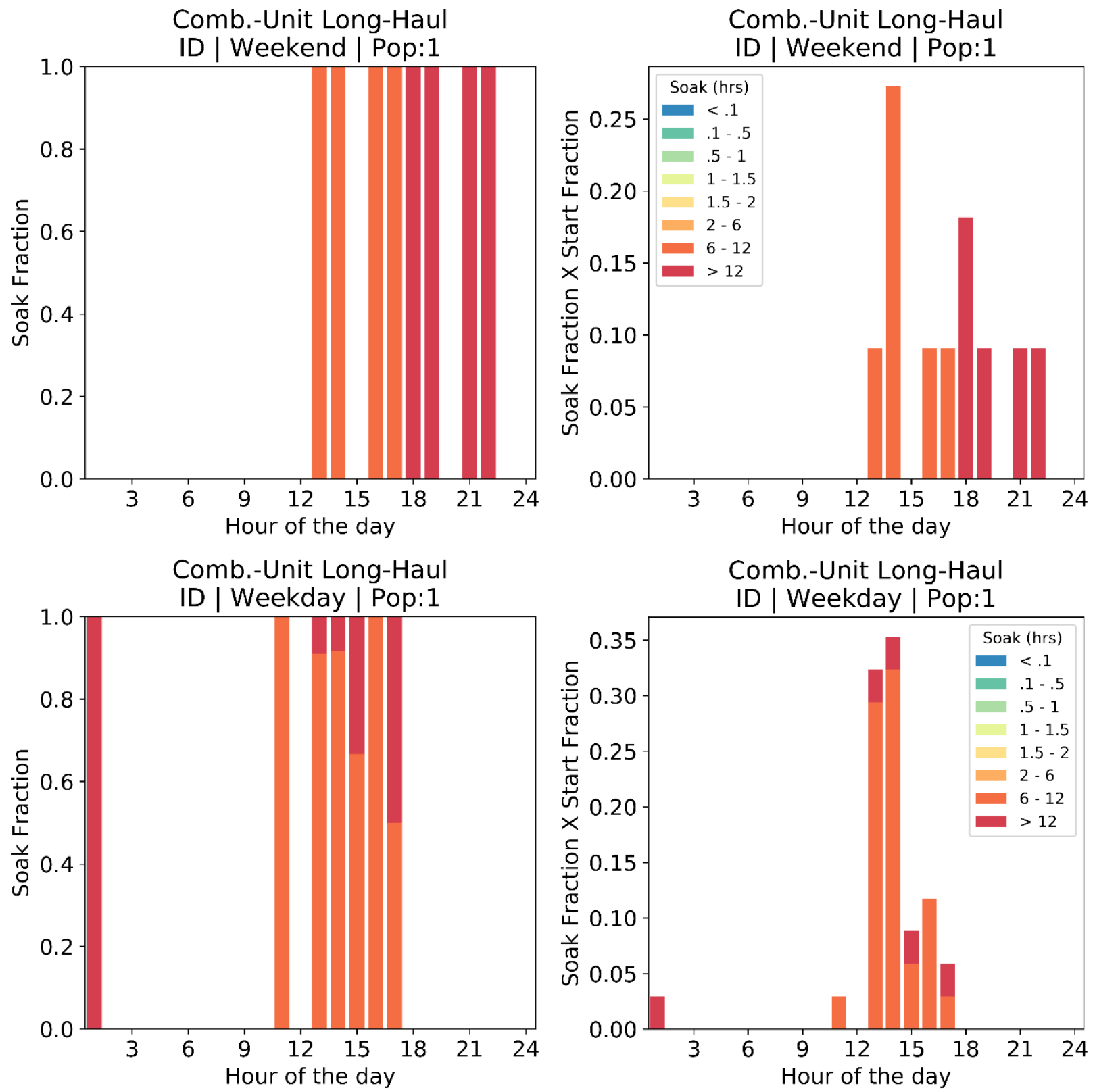

Figure H.39. Soak fraction and start fraction vs. hour of the day for Comb.-Unit Long-Haul - ID 

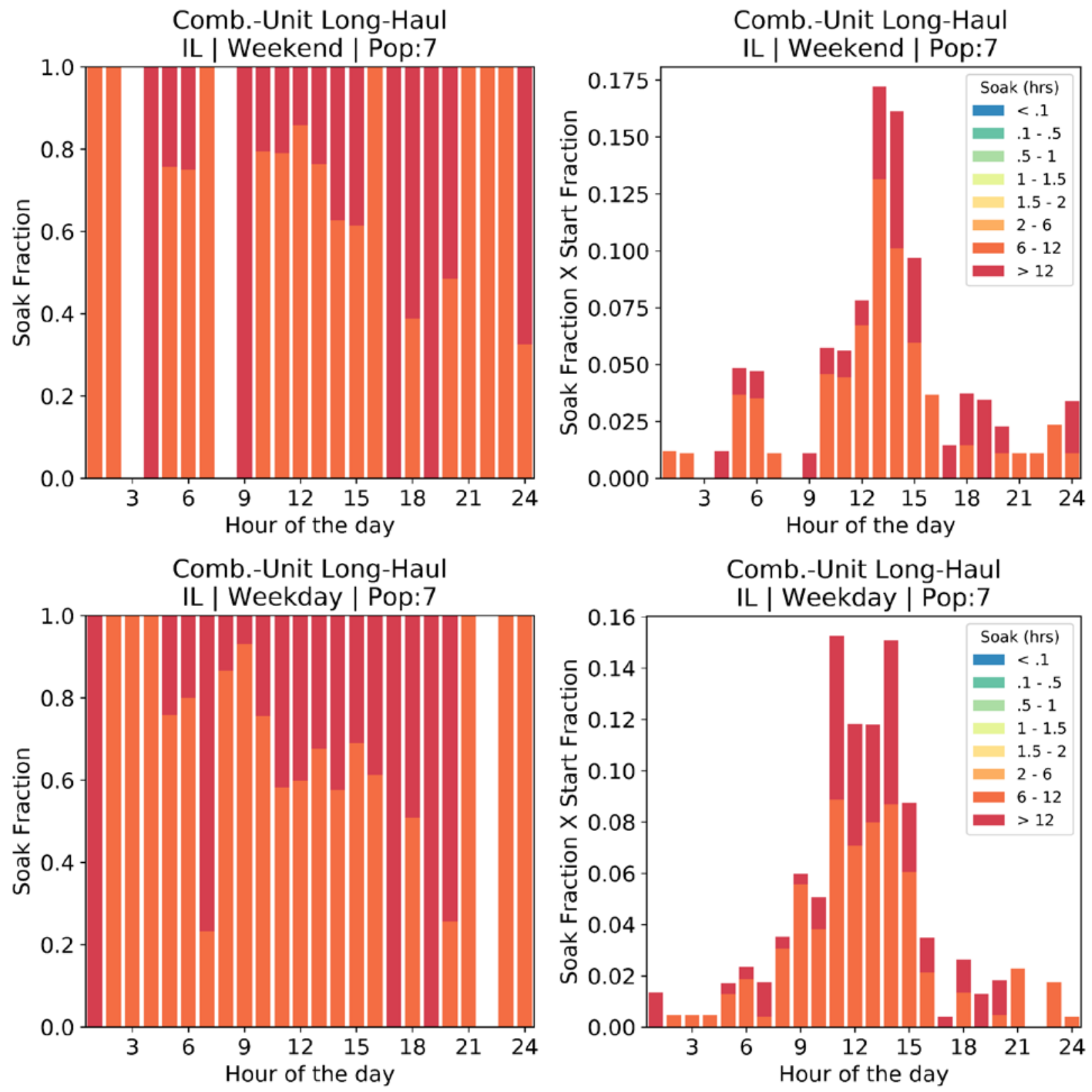

Figure H.40. Soak fraction and start fraction vs. hour of the day for Comb.-Unit Long-Haul - IL 

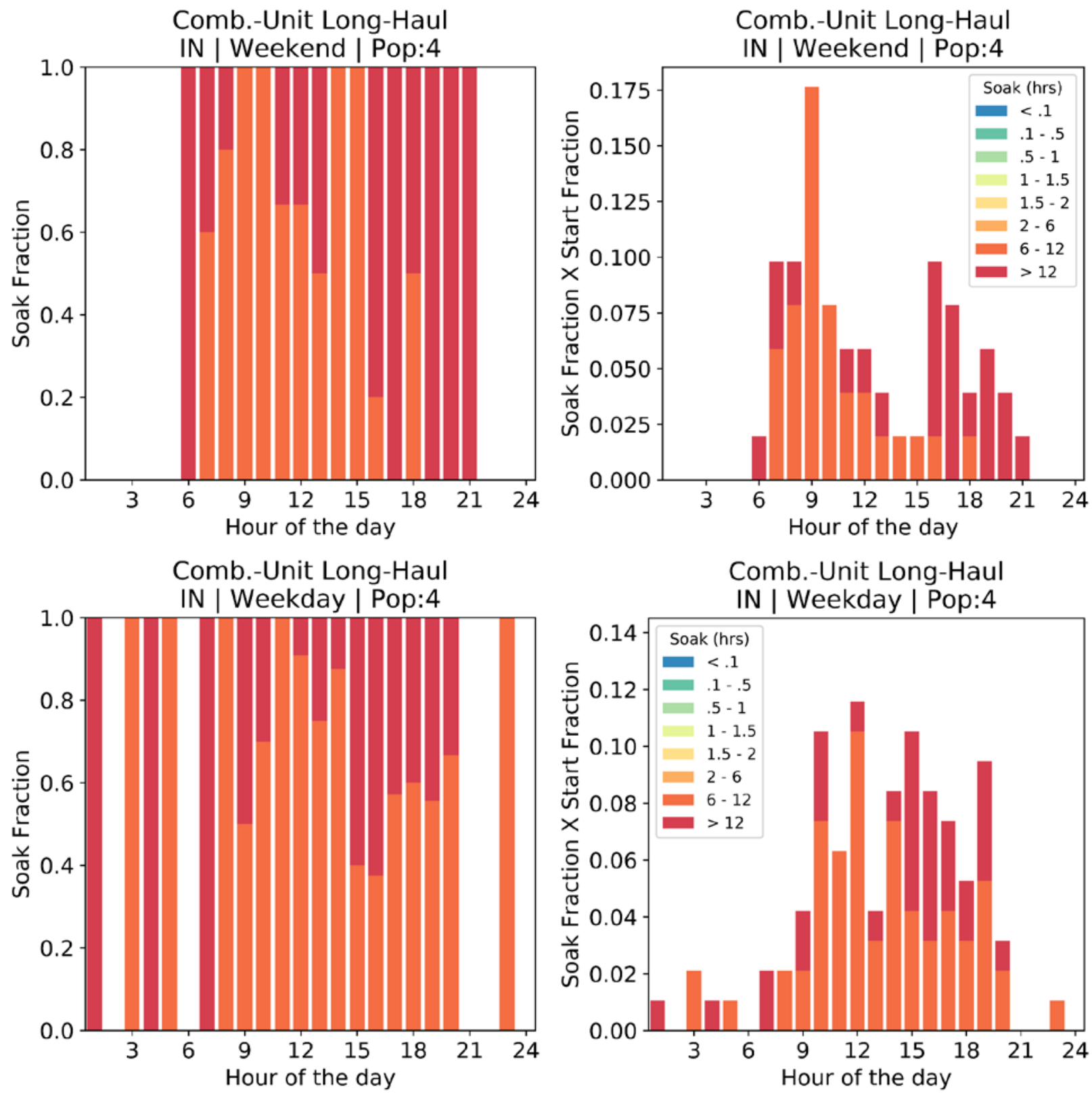

Figure H.41. Soak fraction and start fraction vs. hour of the day for Comb.-Unit Long-Haul - IN 

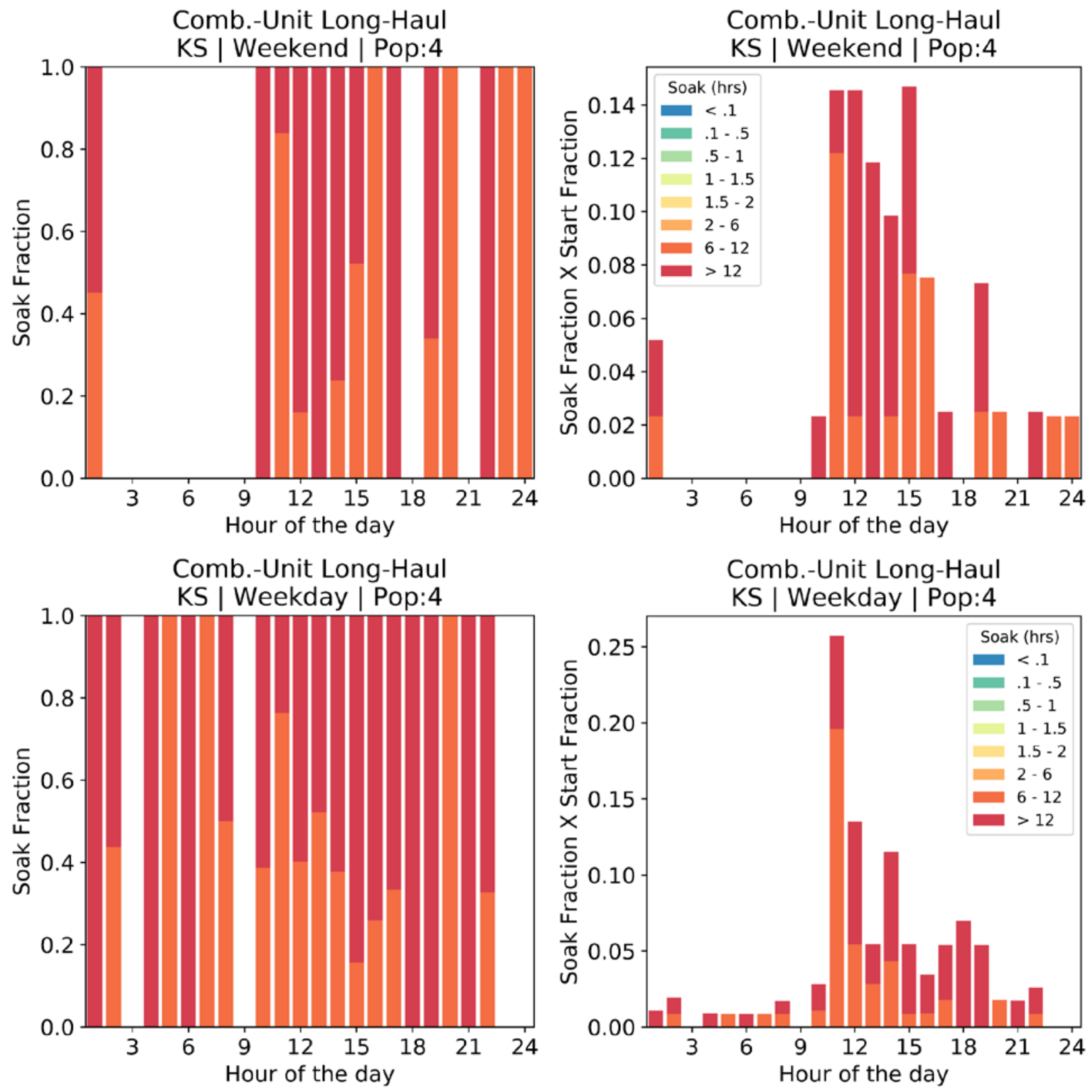

Figure H.42. Soak fraction and start fraction vs. hour of the day for Comb.-Unit Long-Haul - KS 

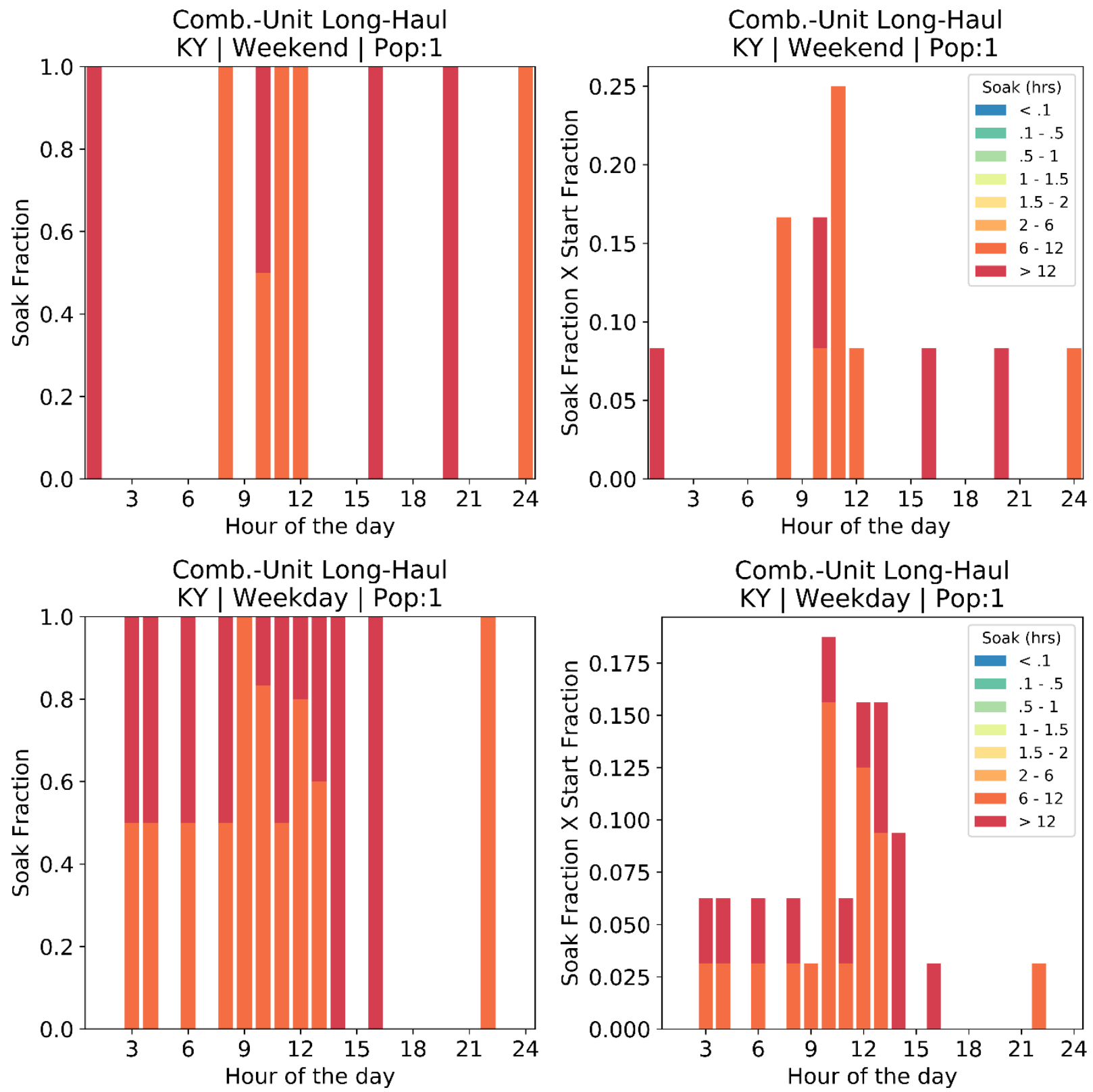

Figure H.43. Soak fraction and start fraction vs. hour of the day for Comb.-Unit Long-Haul - KY 

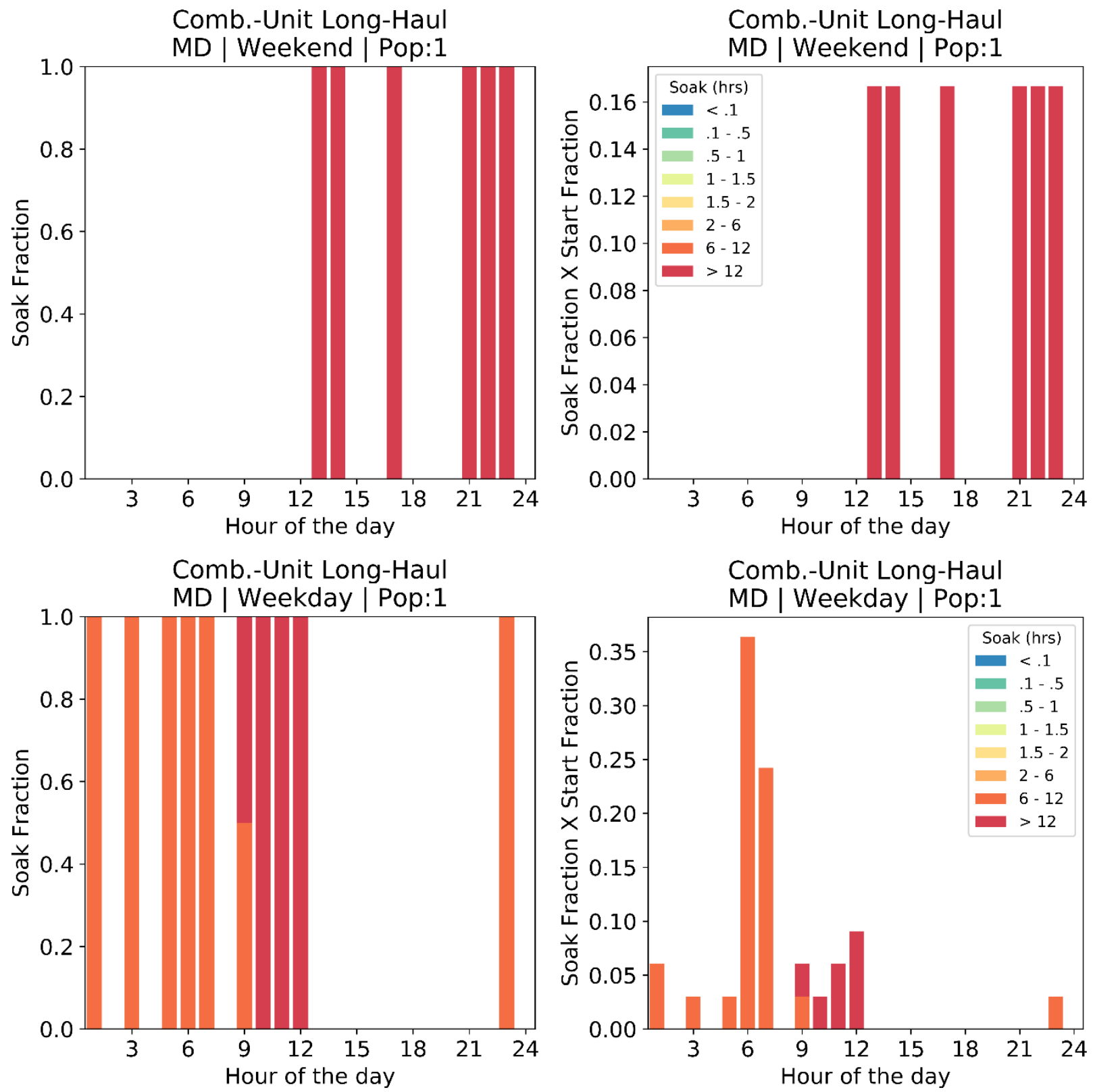

Figure H.44. Soak fraction and start fraction vs. hour of the day for Comb.-Unit Long-Haul - MD 

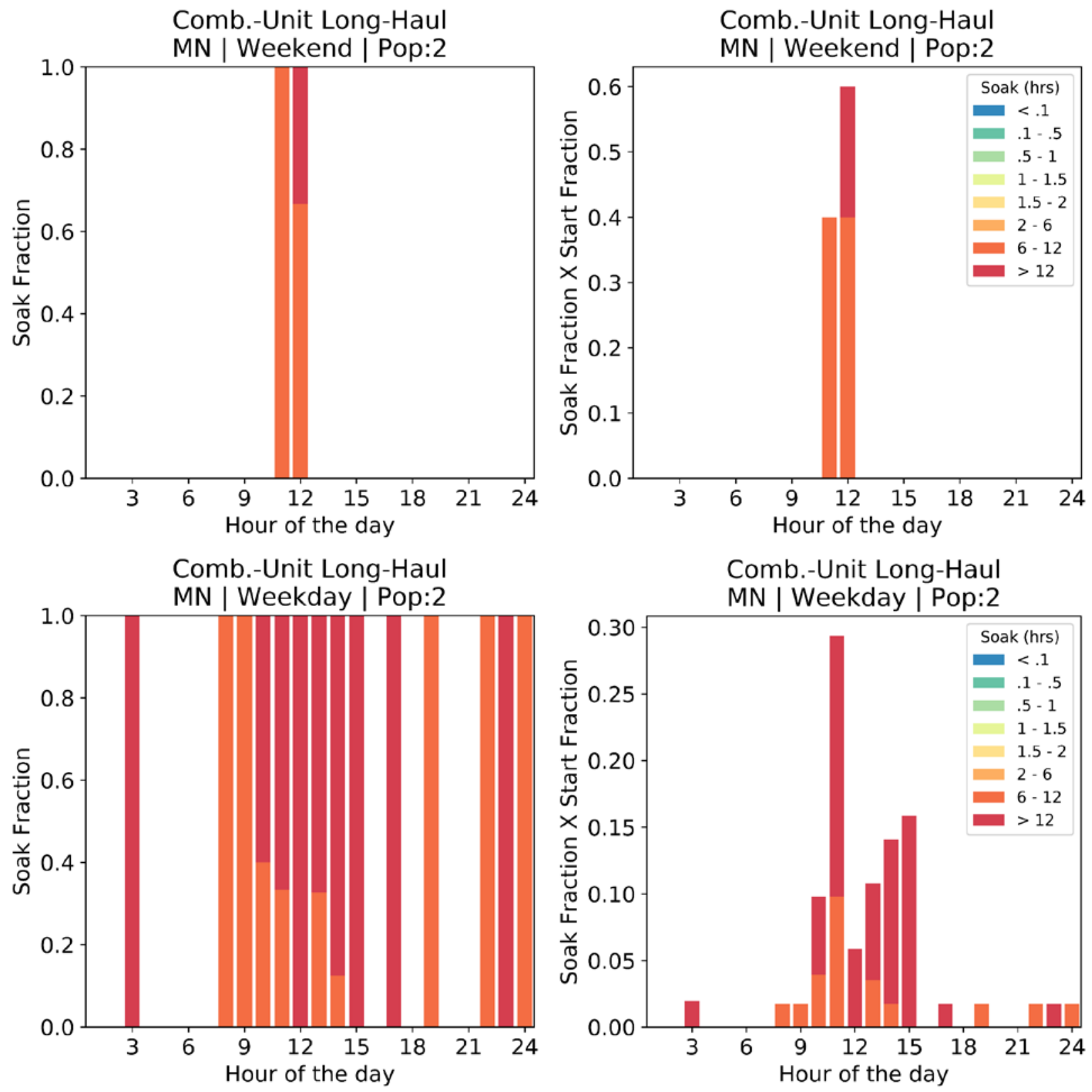

Figure H.45. Soak fraction and start fraction vs. hour of the day for Comb.-Unit Long-Haul - MN 

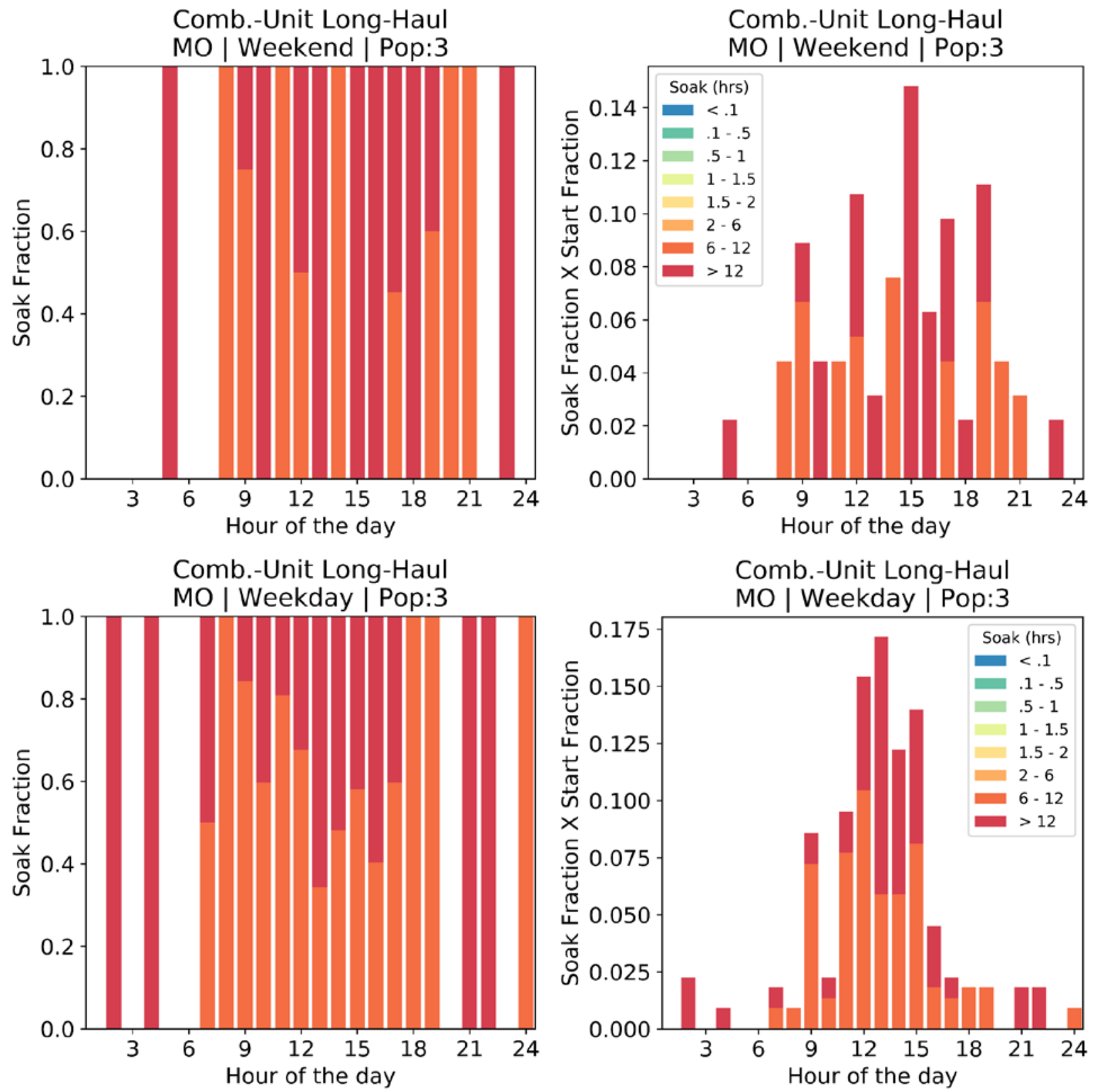

Figure H.46. Soak fraction and start fraction vs. hour of the day for Comb.-Unit Long-Haul - MO 

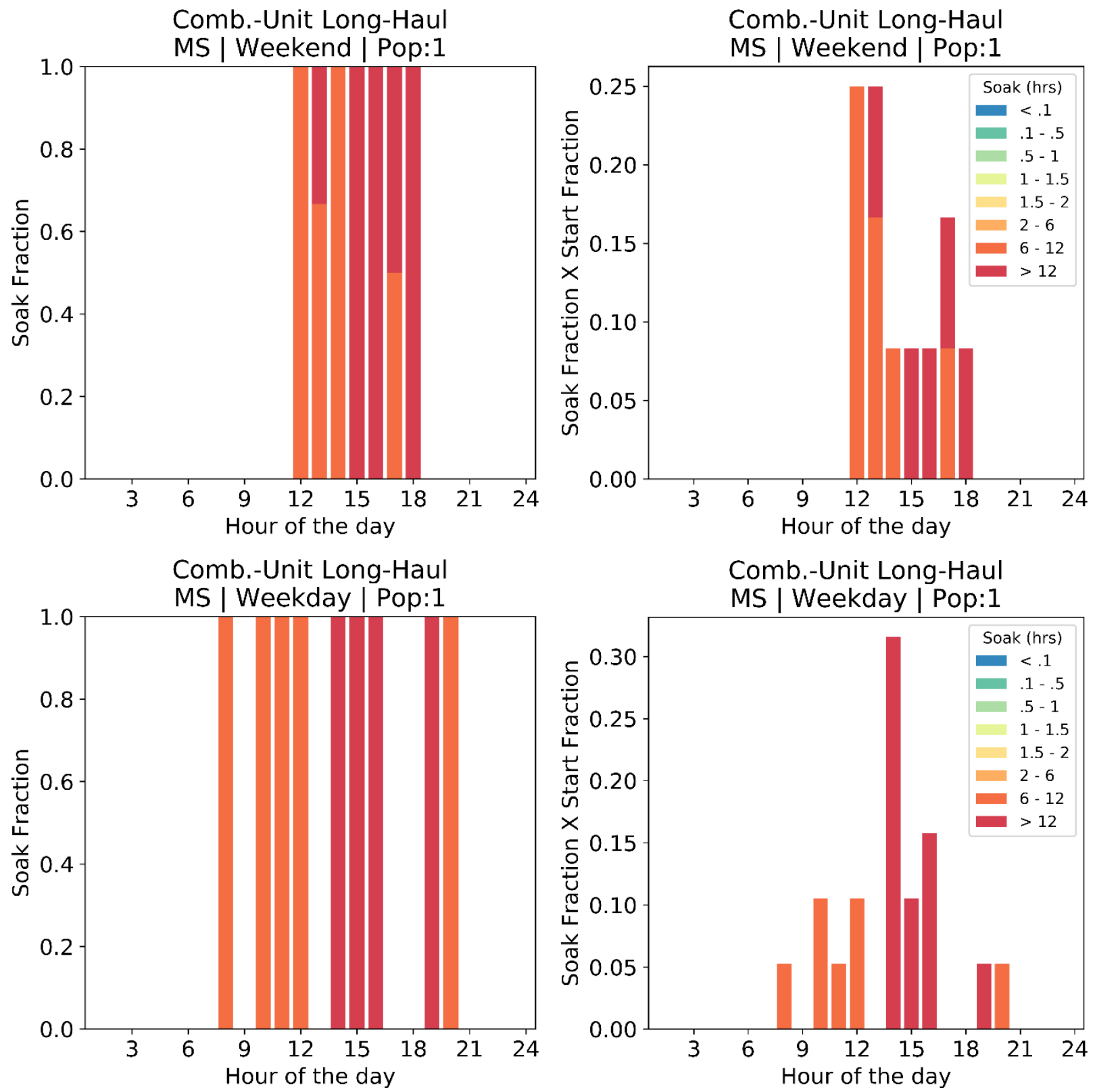

Comb.-Unit Long-Haul

MS | Weekday | Pop:1

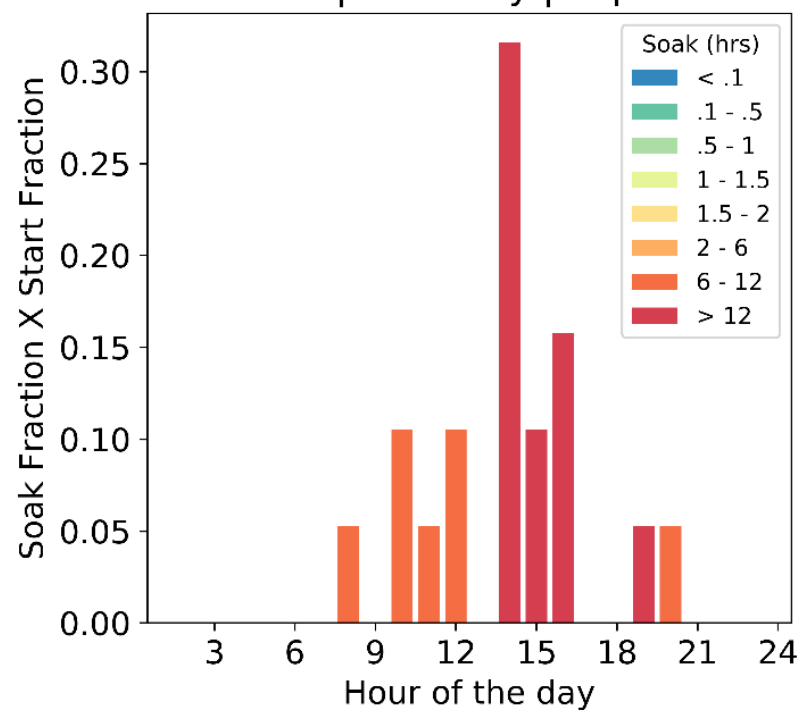

Figure H.47. Soak fraction and start fraction vs. hour of the day for Comb.-Unit Long-Haul - MS 

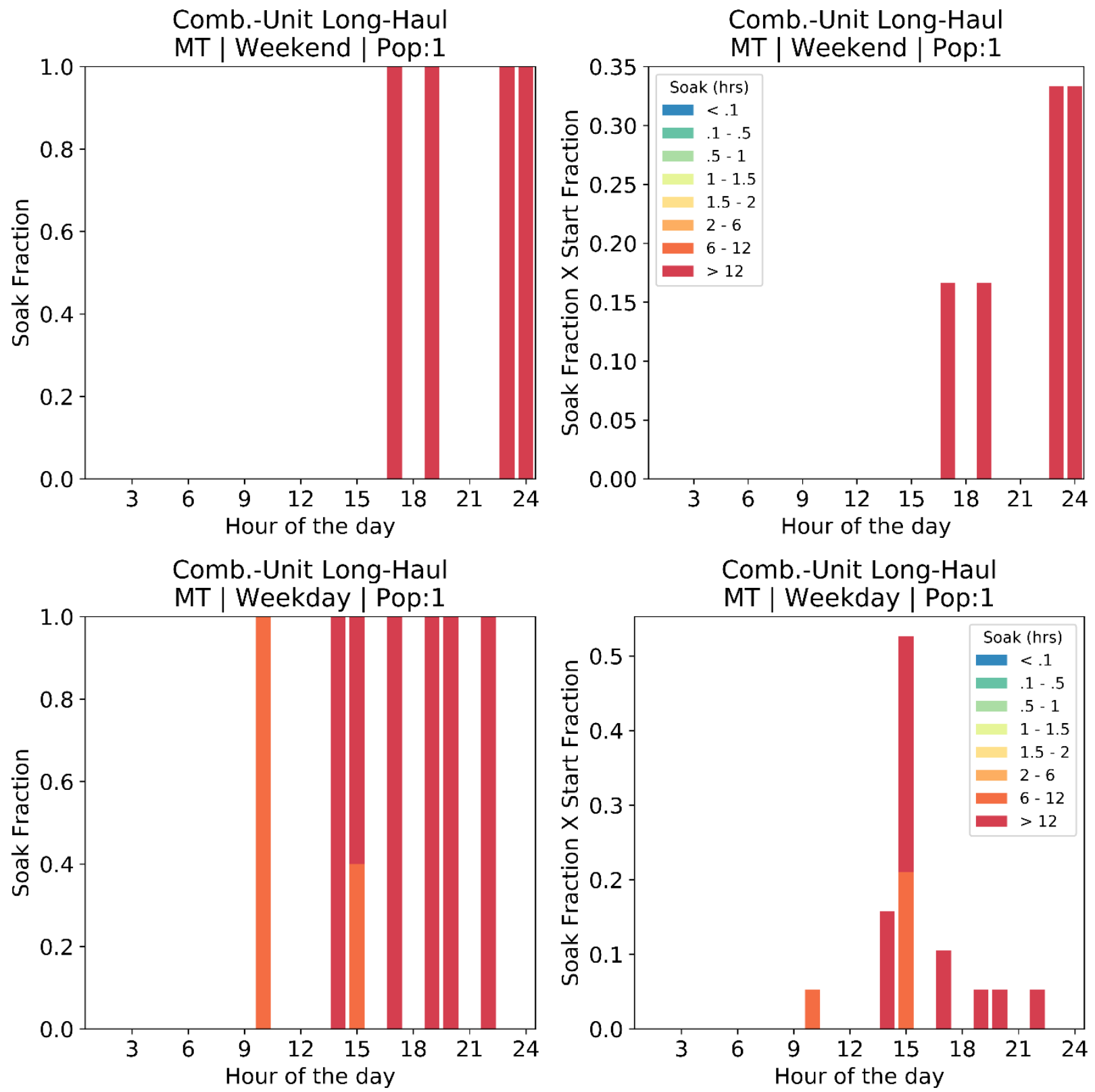

Figure H.48. Soak fraction and start fraction vs. hour of the day for Comb.-Unit Long-Haul - MT 

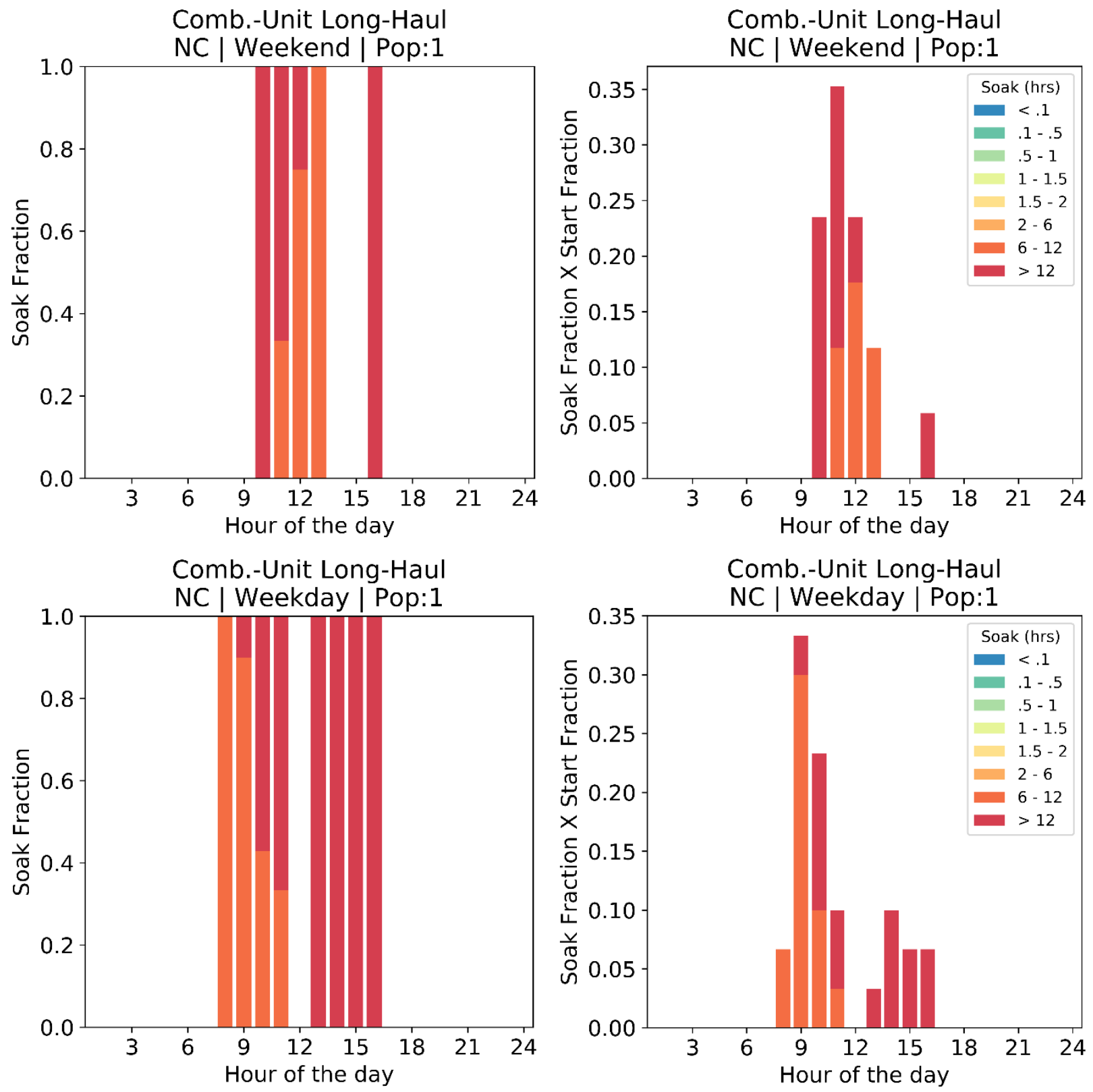

Figure H.49. Soak fraction and start fraction vs. hour of the day for Comb.-Unit Long-Haul - NC 

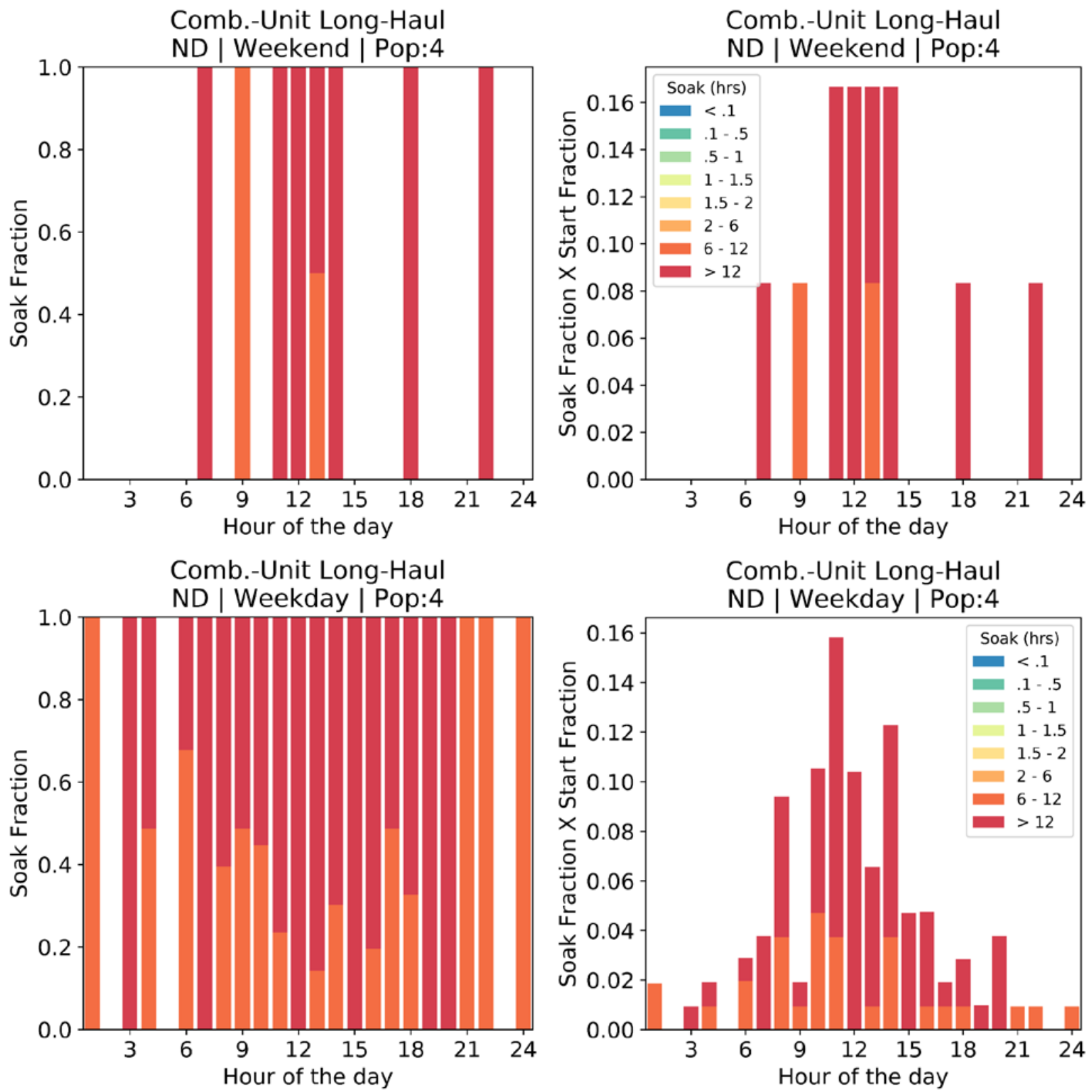

Figure H.50. Soak fraction and start fraction vs. hour of the day for Comb.-Unit Long-Haul - ND 

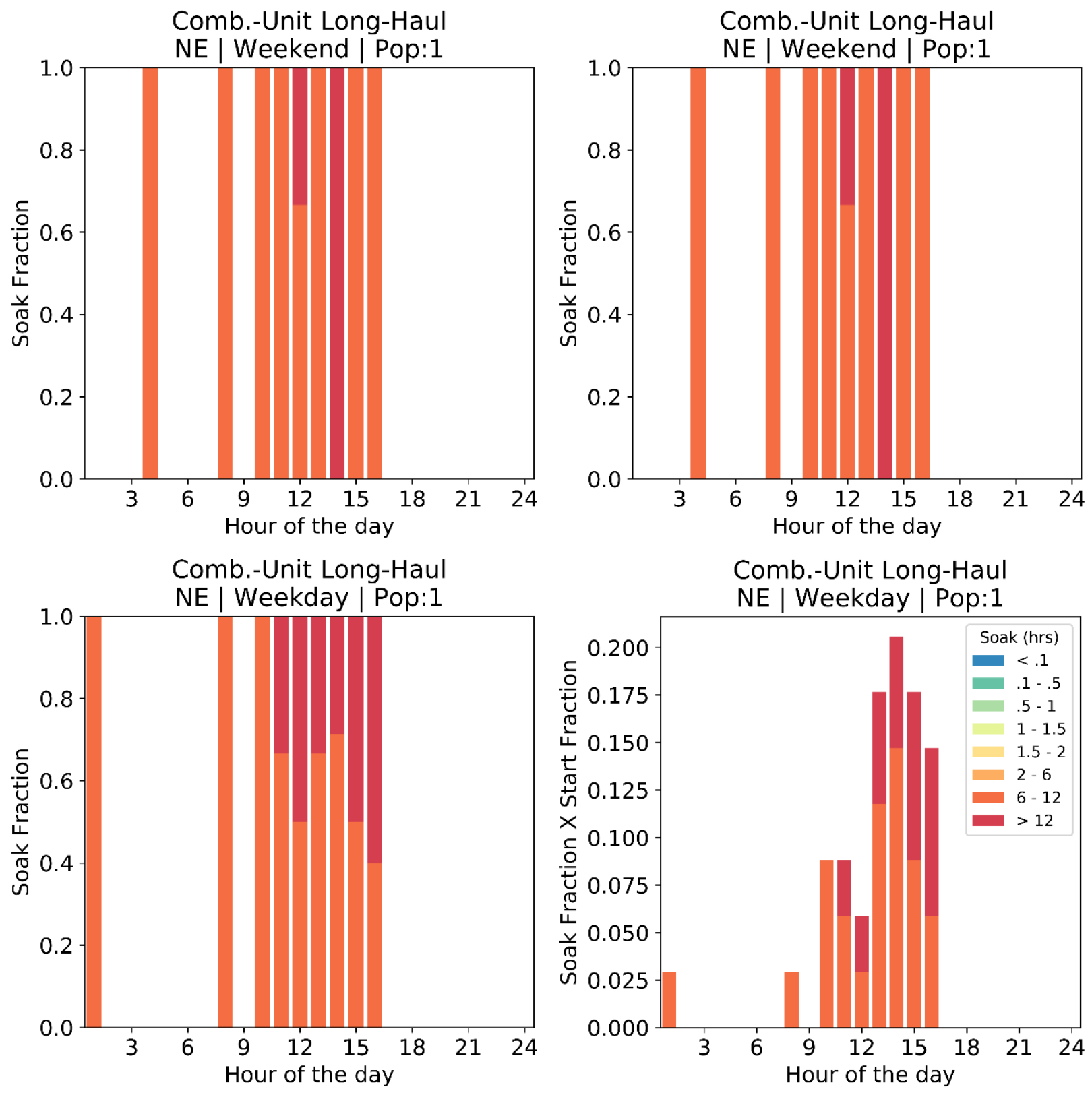

Figure H.51. Soak fraction and start fraction vs. hour of the day for Comb.-Unit Long-Haul - NE 

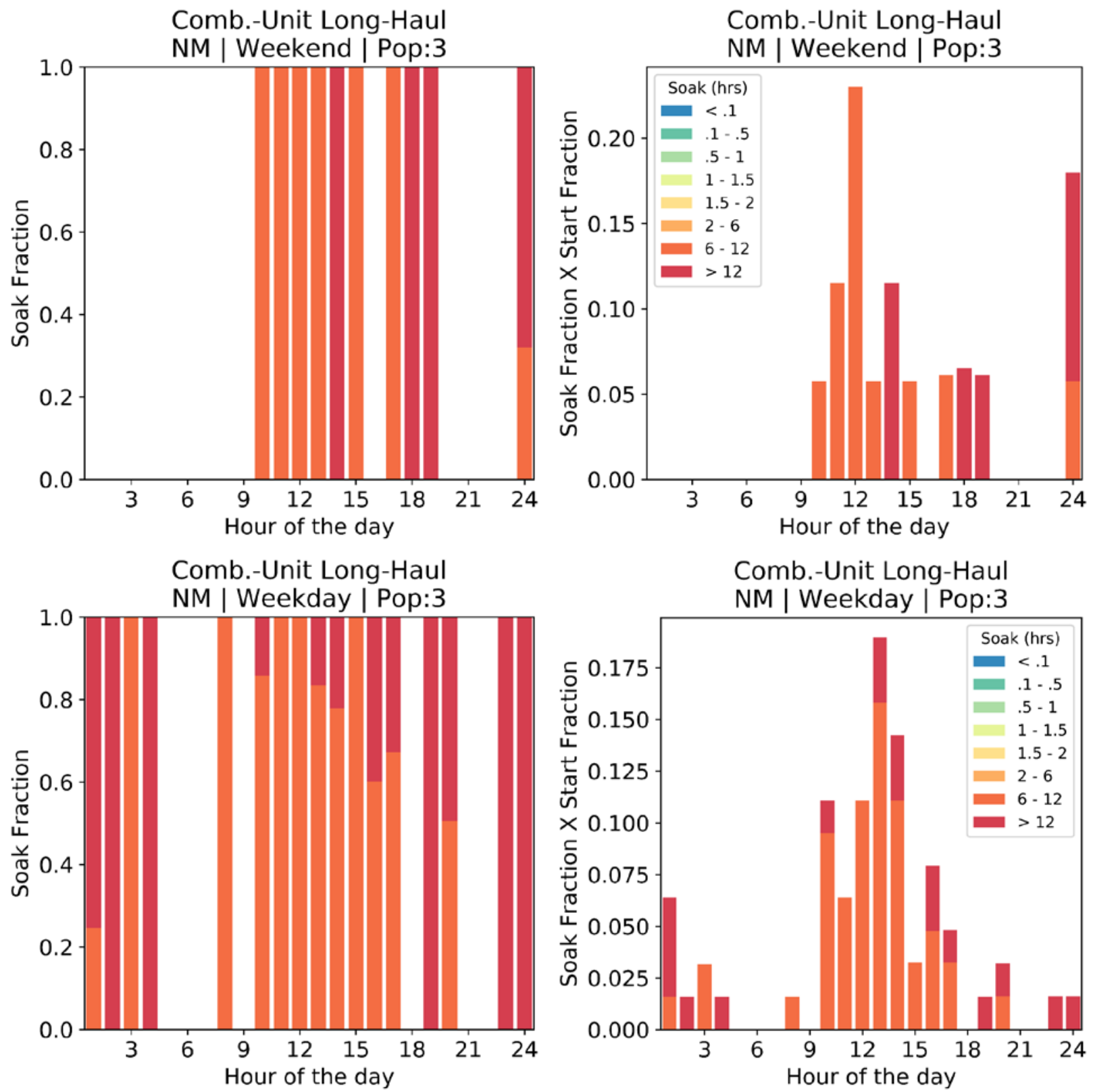

Figure H.52. Soak fraction and start fraction vs. hour of the day for Comb.-Unit Long-Haul - NM 

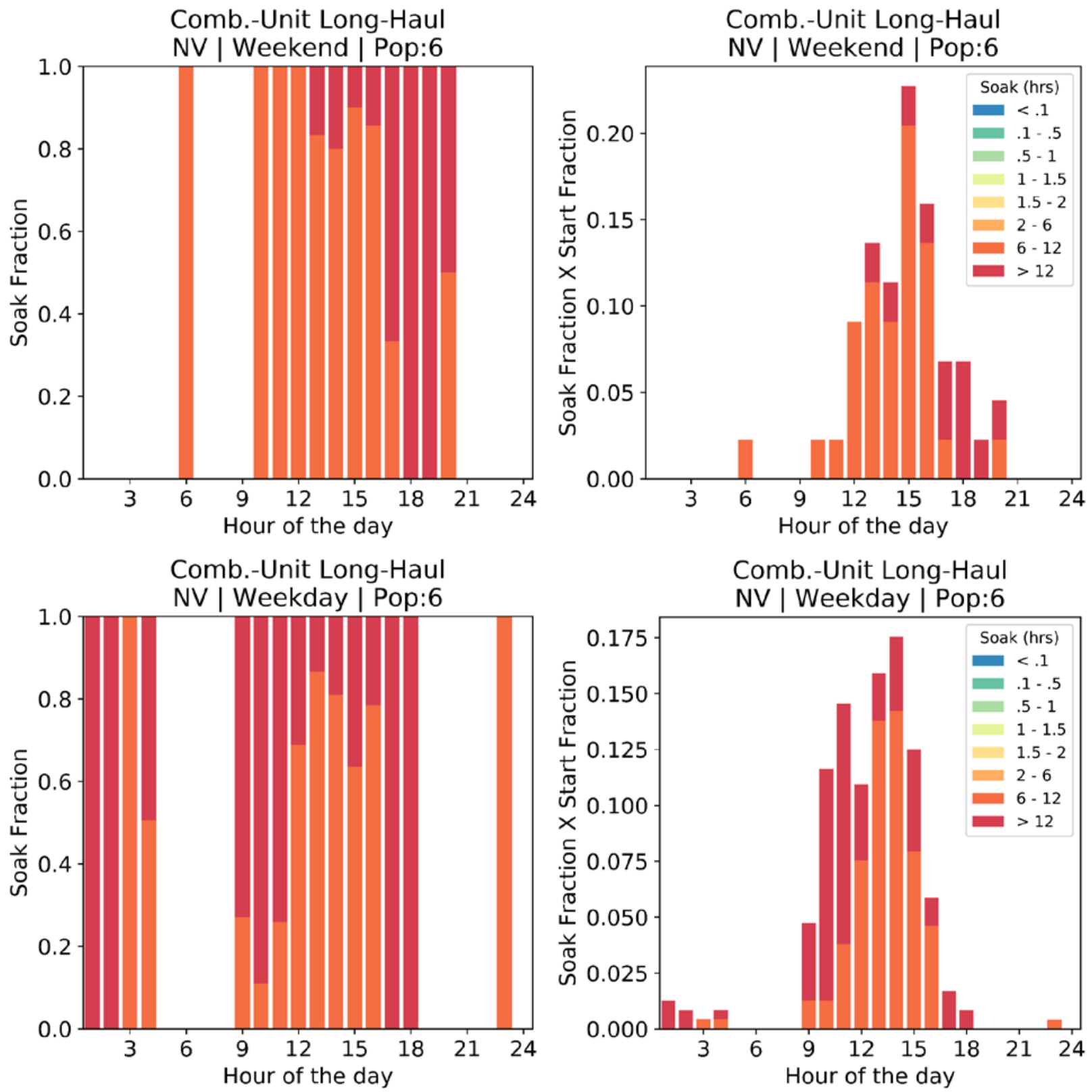

Figure H.53. Soak fraction and start fraction vs. hour of the day for Comb.-Unit Long-Haul - NV 

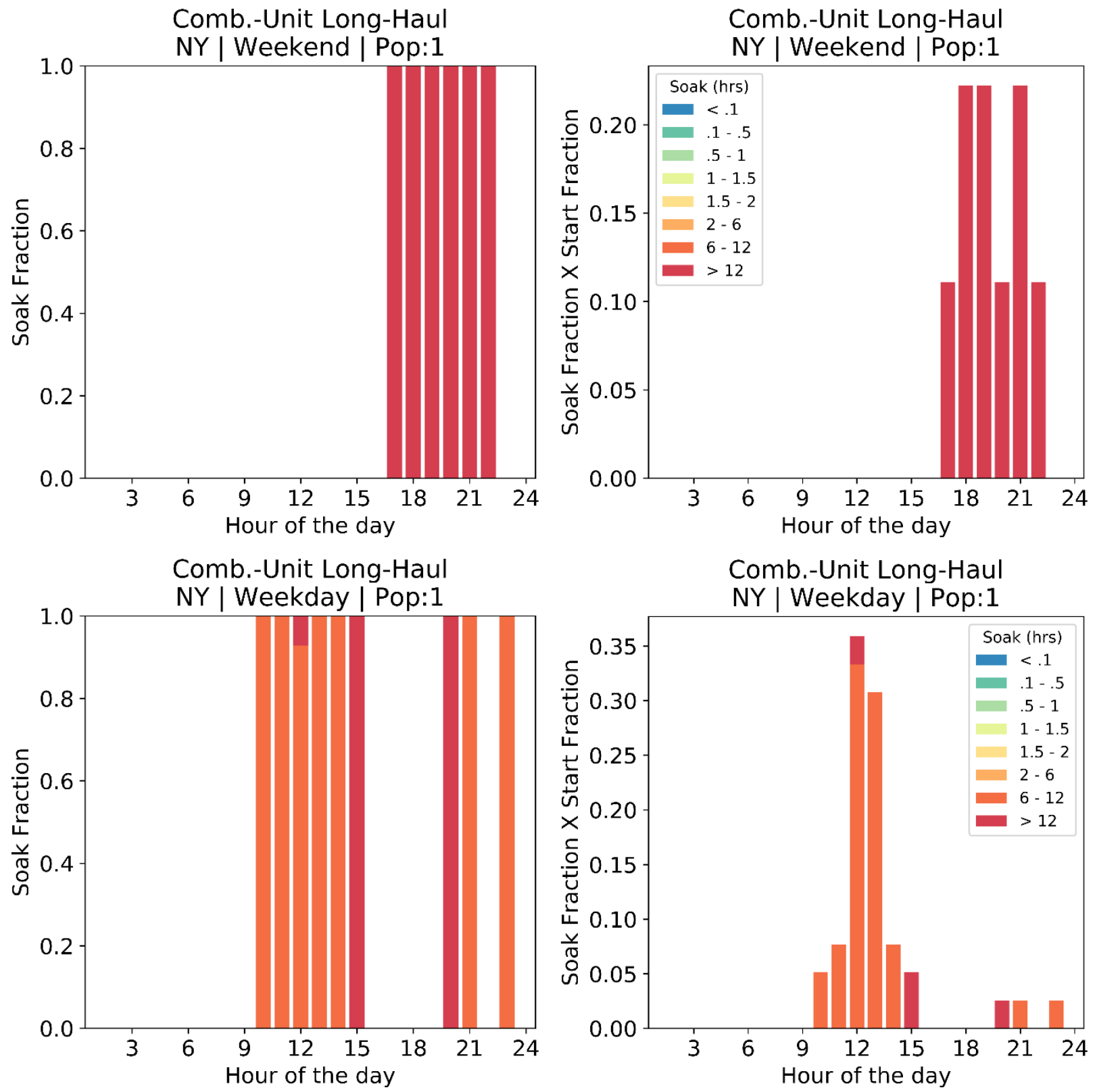

Figure H.54. Soak fraction and start fraction vs. hour of the day for Comb.-Unit Long-Haul - NY 

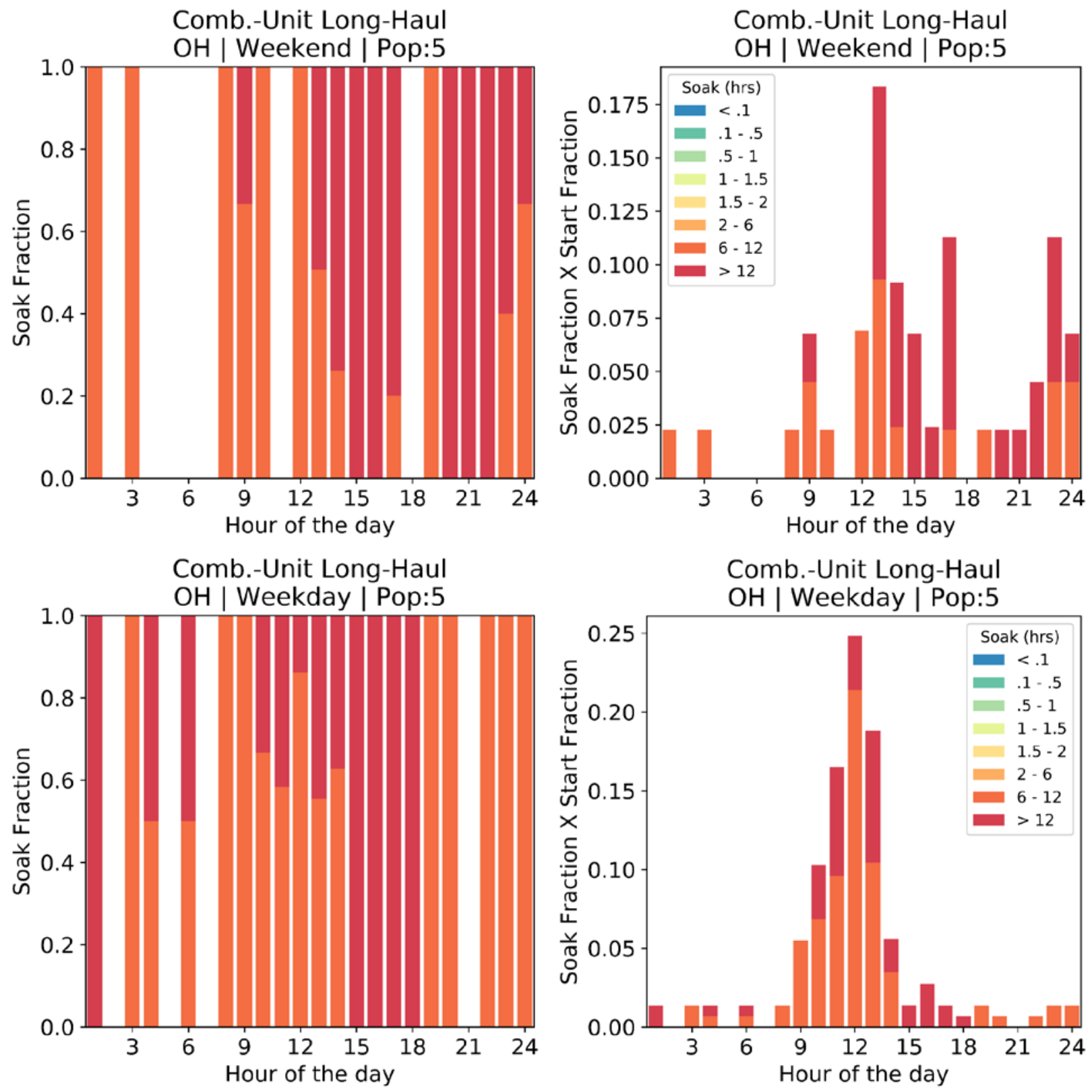

Figure H.55. Soak fraction and start fraction vs. hour of the day for Comb.-Unit Long-Haul - OH 

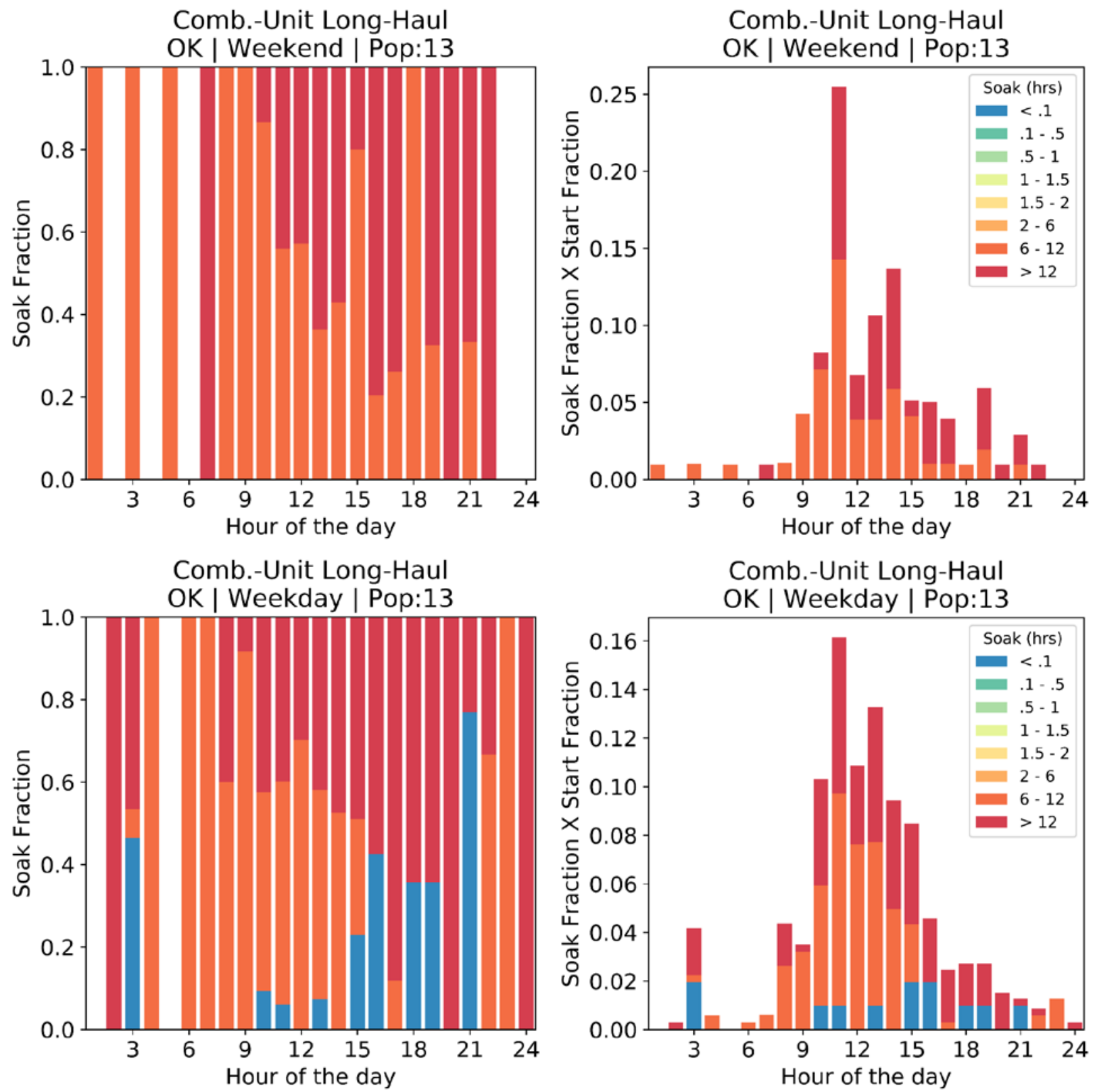

Figure H.56. Soak fraction and start fraction vs. hour of the day for Comb.-Unit Long-Haul - OK 

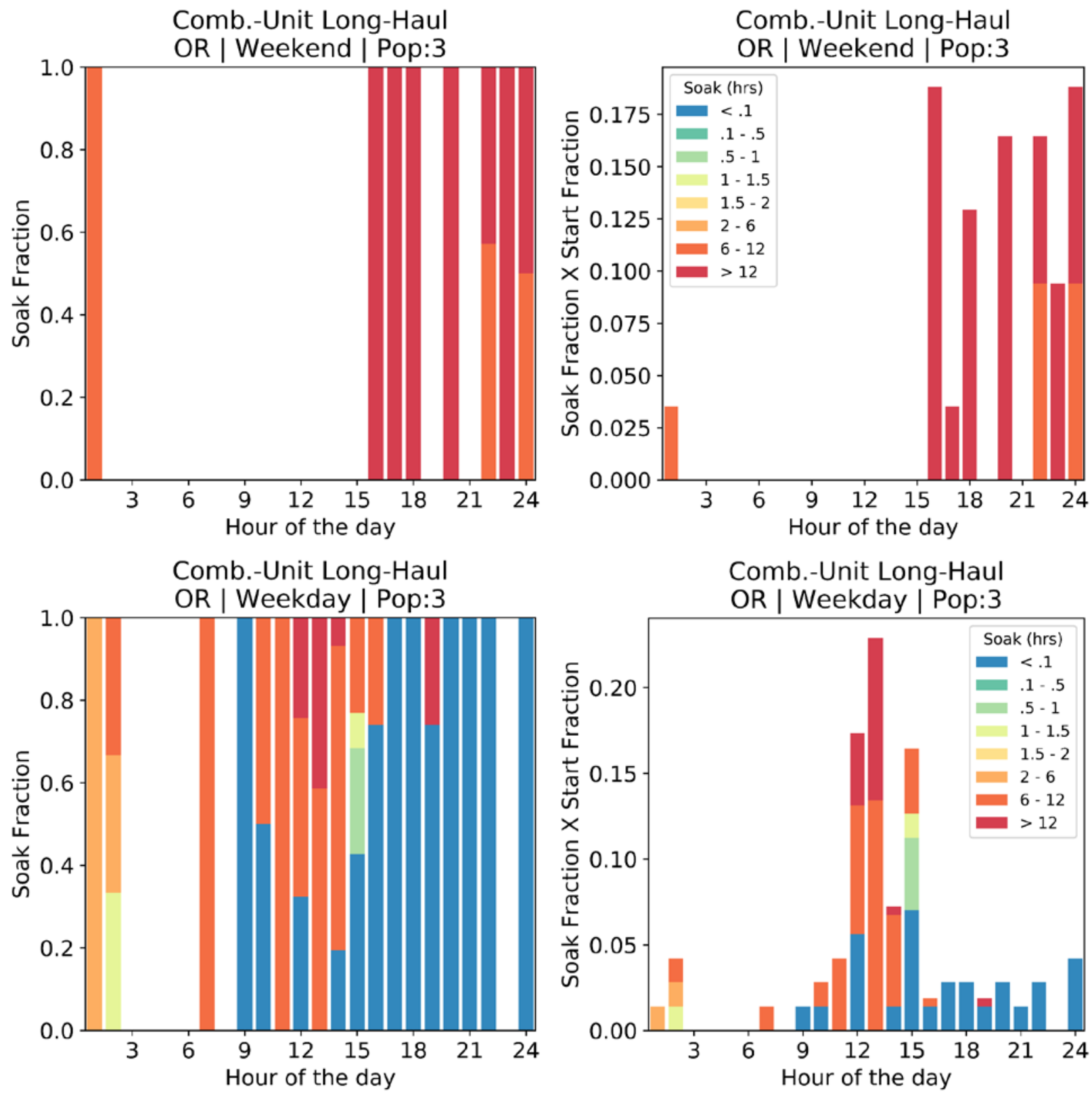

Figure H.57. Soak fraction and start fraction vs. hour of the day for Comb.-Unit Long-Haul - OR 

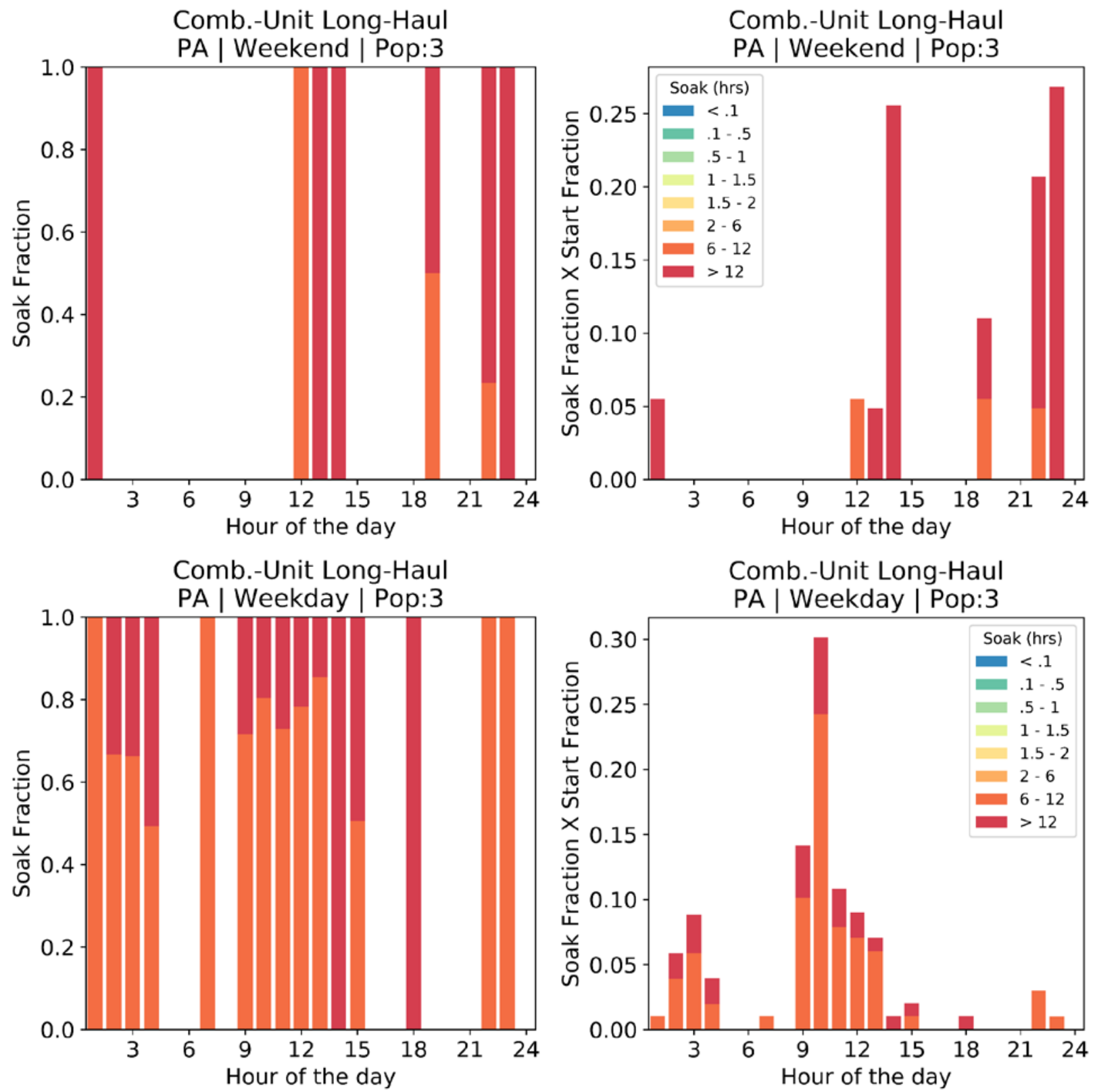

Figure H.58. Soak fraction and start fraction vs. hour of the day for Comb.-Unit Long-Haul - PA 

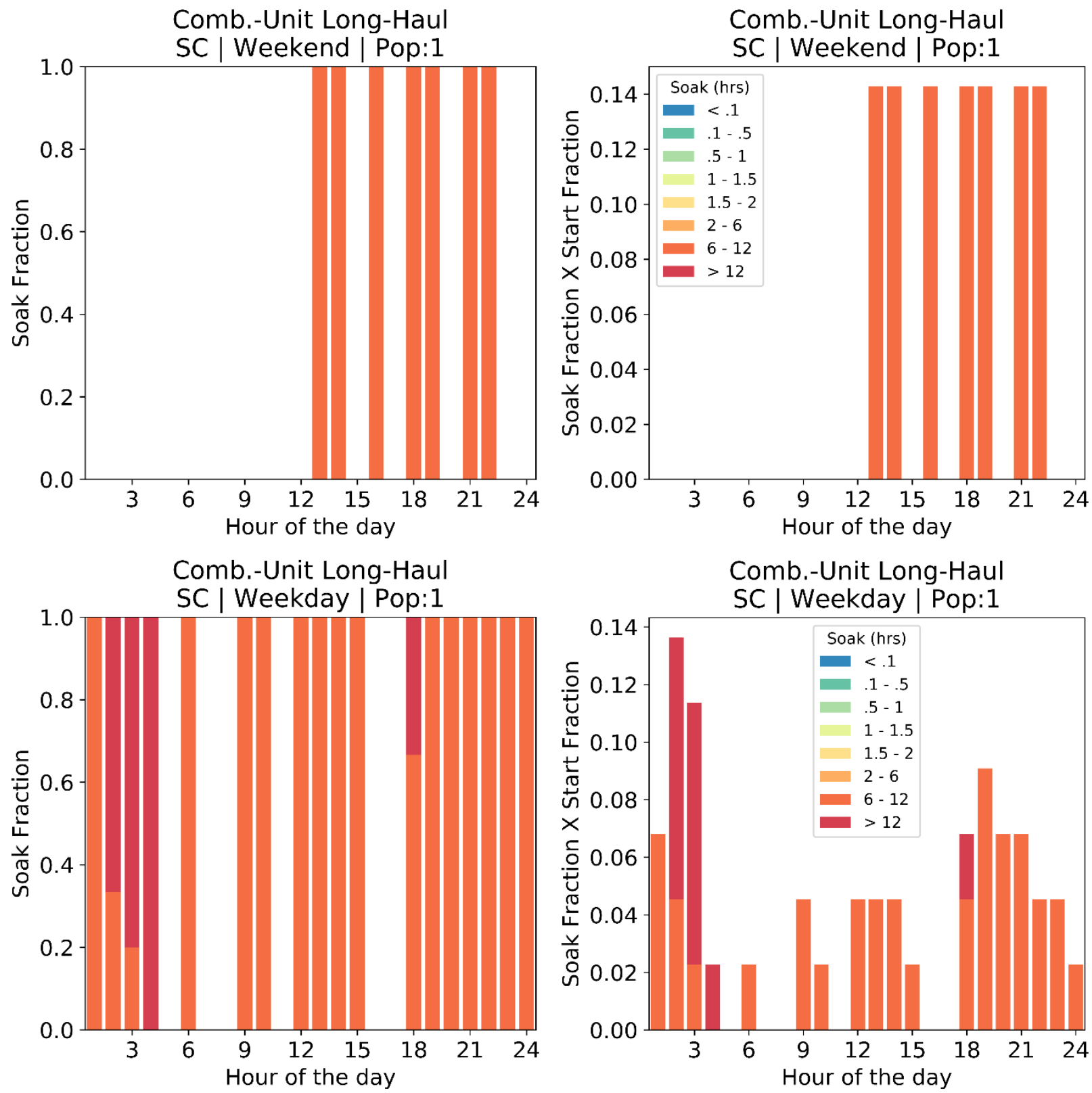

Figure H.59. Soak fraction and start fraction vs. hour of the day for Comb.-Unit Long-Haul - SC 

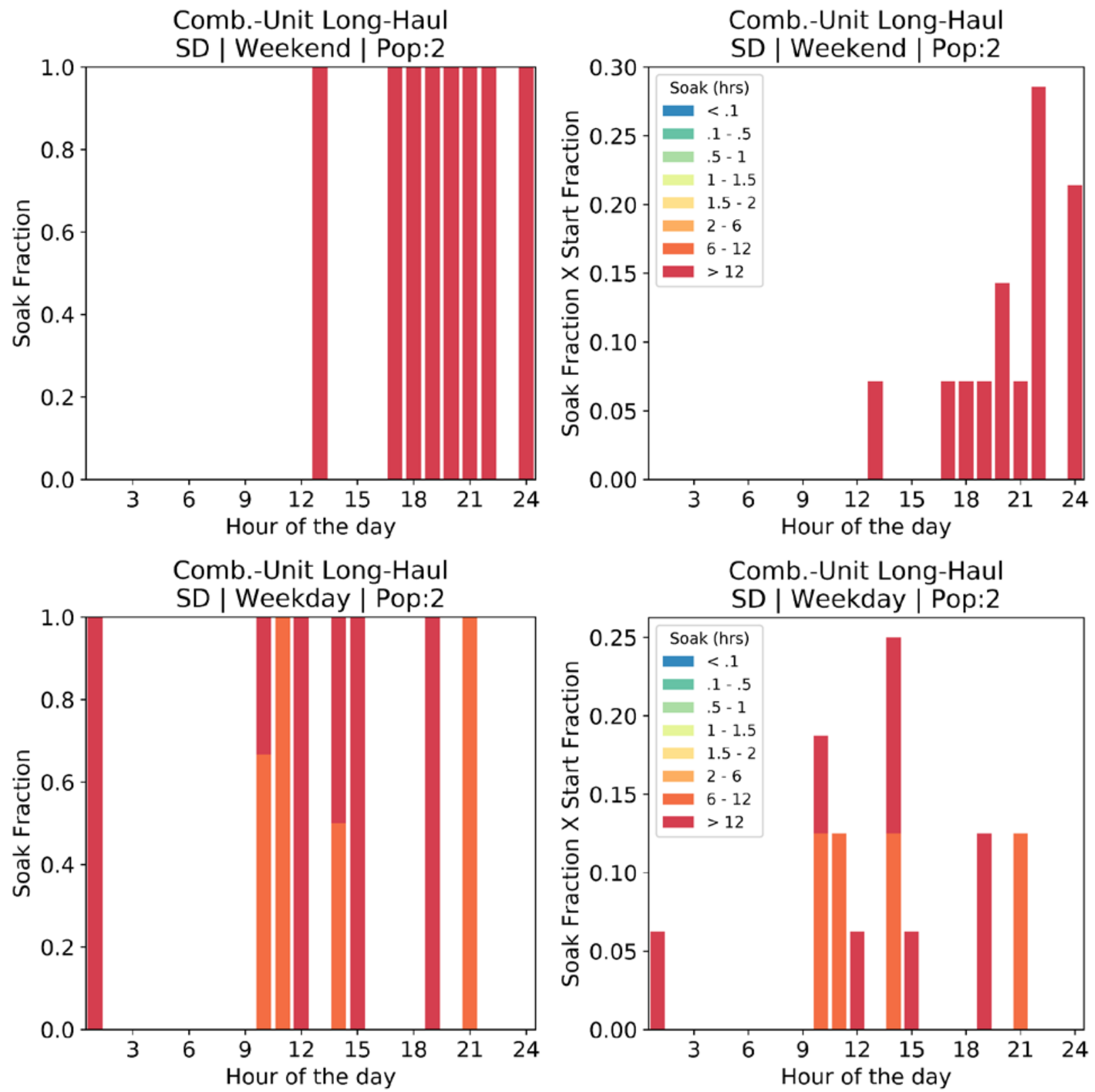

Figure H.60. Soak fraction and start fraction vs. hour of the day for Comb.-Unit Long-Haul - SD 


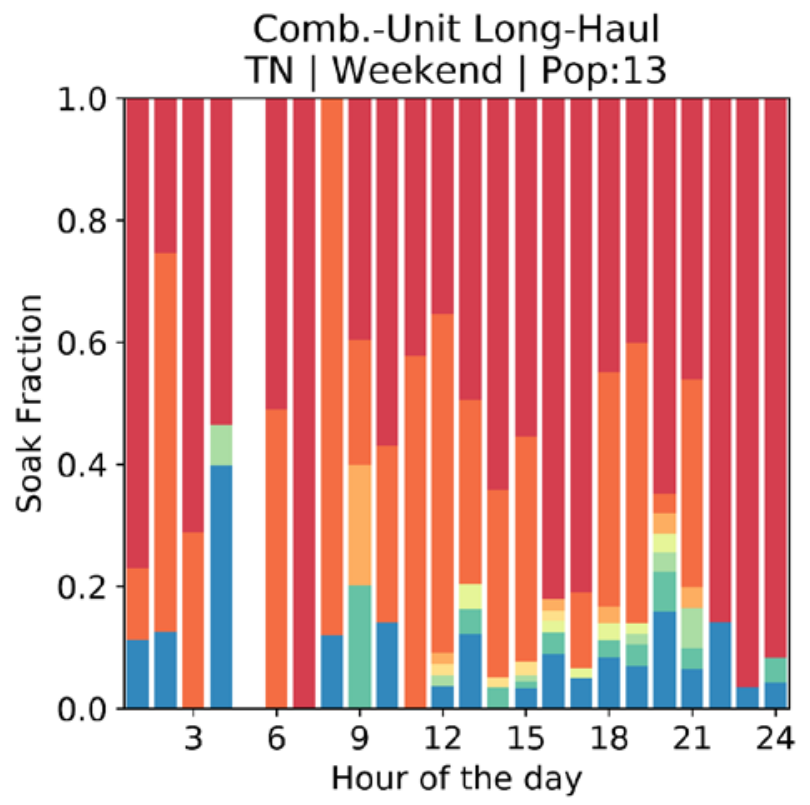

Comb.-Unit Long-Haul TN | Weekday | Pop:13

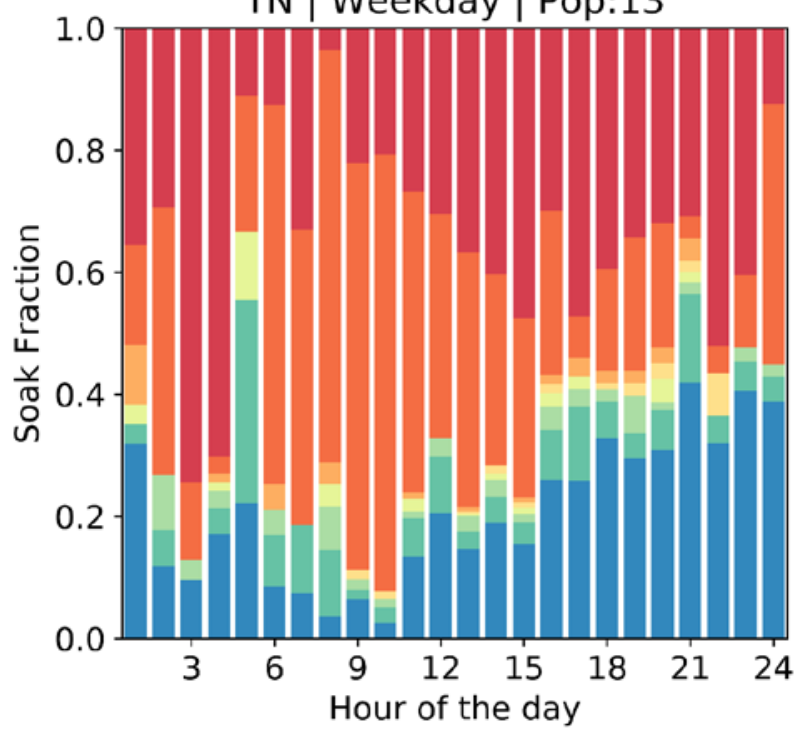

Comb.-Unit Long-Haul

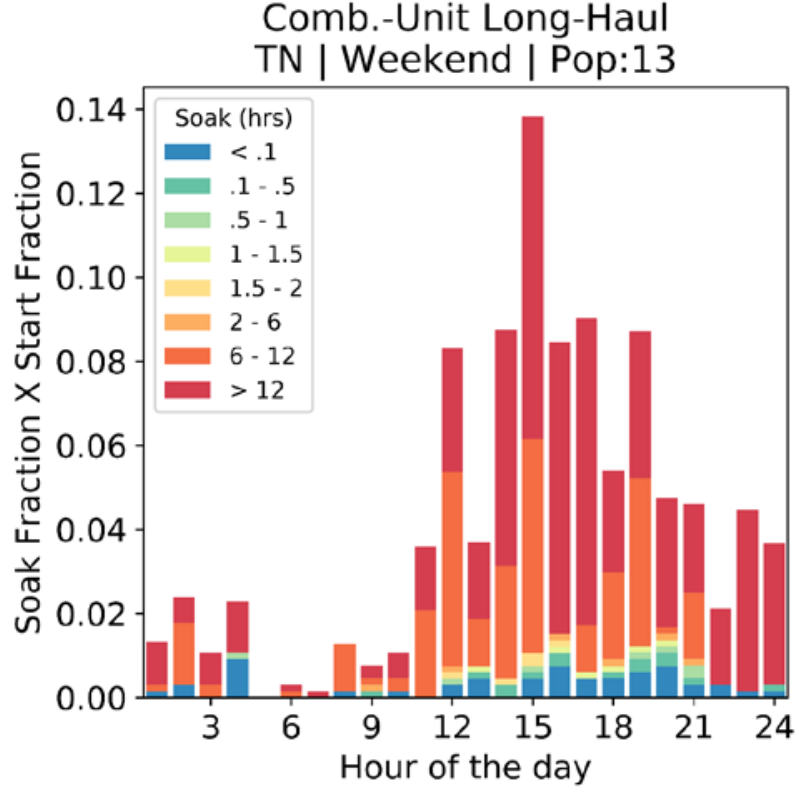

Comb.-Unit Long-Haul TN | Weekday | Pop:13

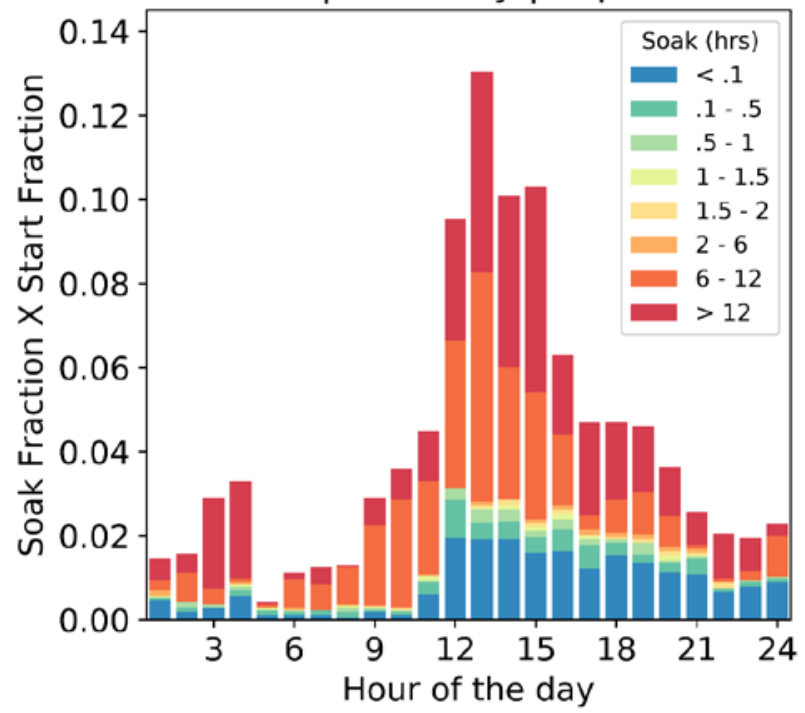

Figure H.61. Soak fraction and start fraction vs. hour of the day for Comb.-Unit Long-Haul - TN 

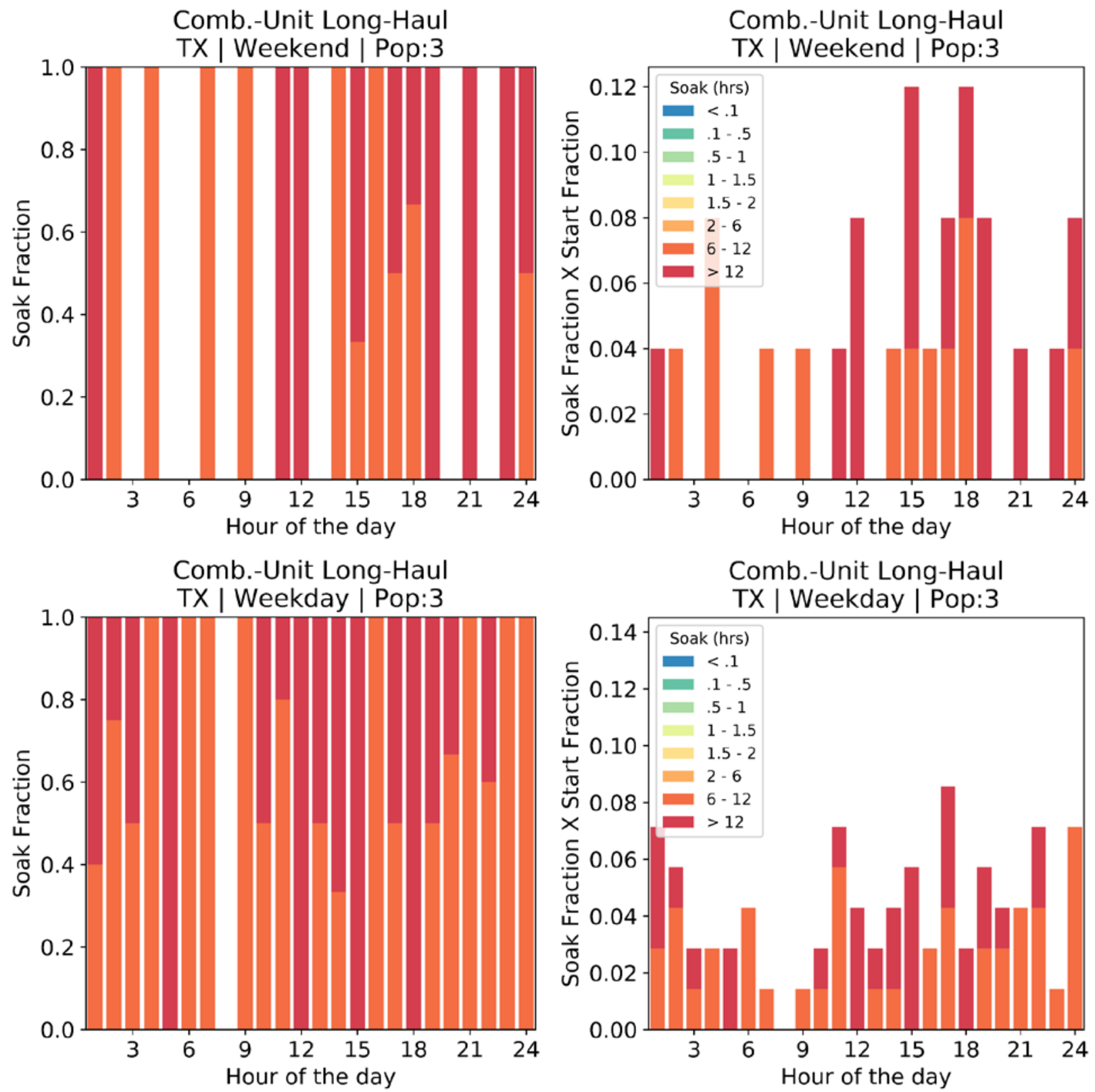

Figure H.62. Soak fraction and start fraction vs. hour of the day for Comb.-Unit Long-Haul - TX 

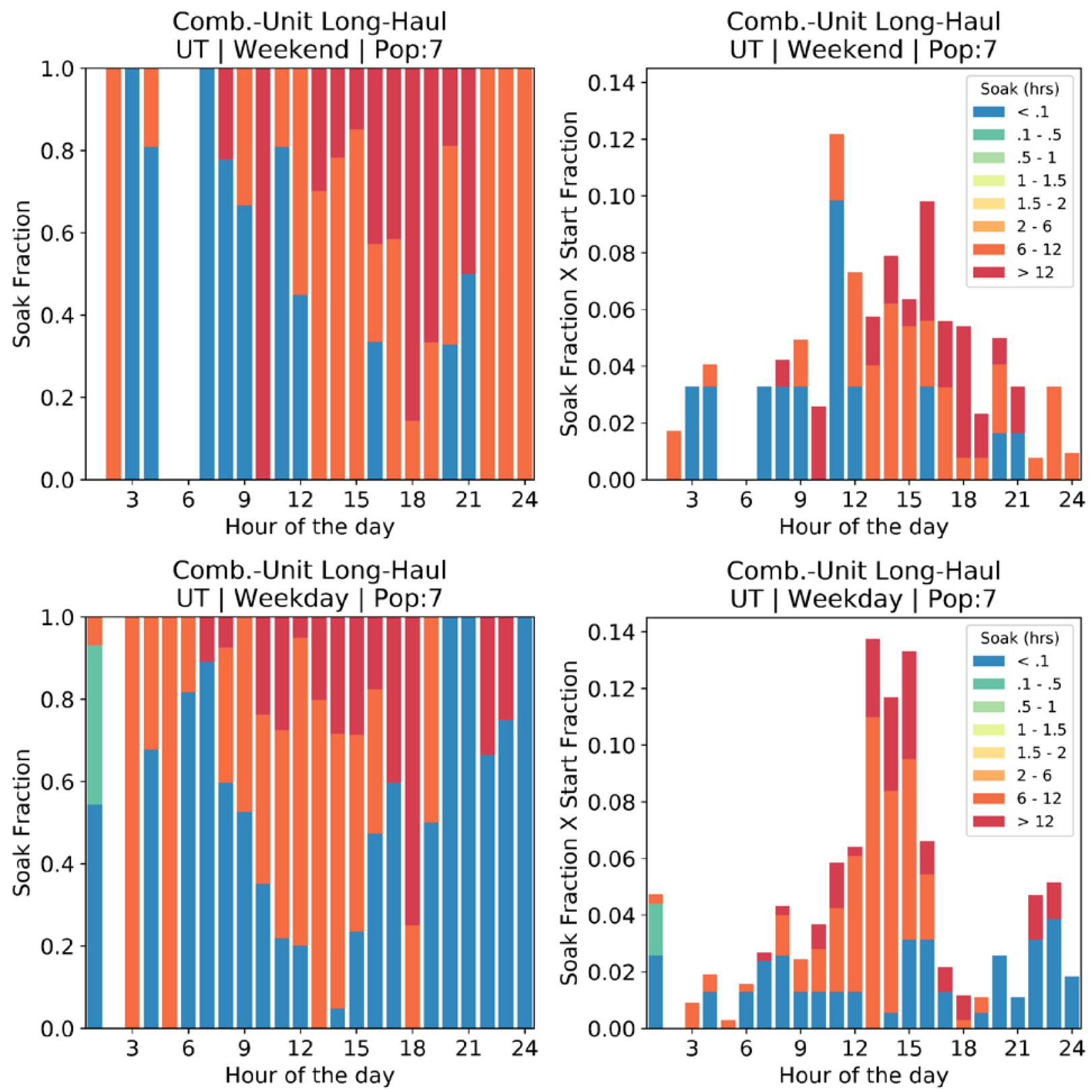

Figure H.63. Soak fraction and start fraction vs. hour of the day for Comb.-Unit Long-Haul - UT 

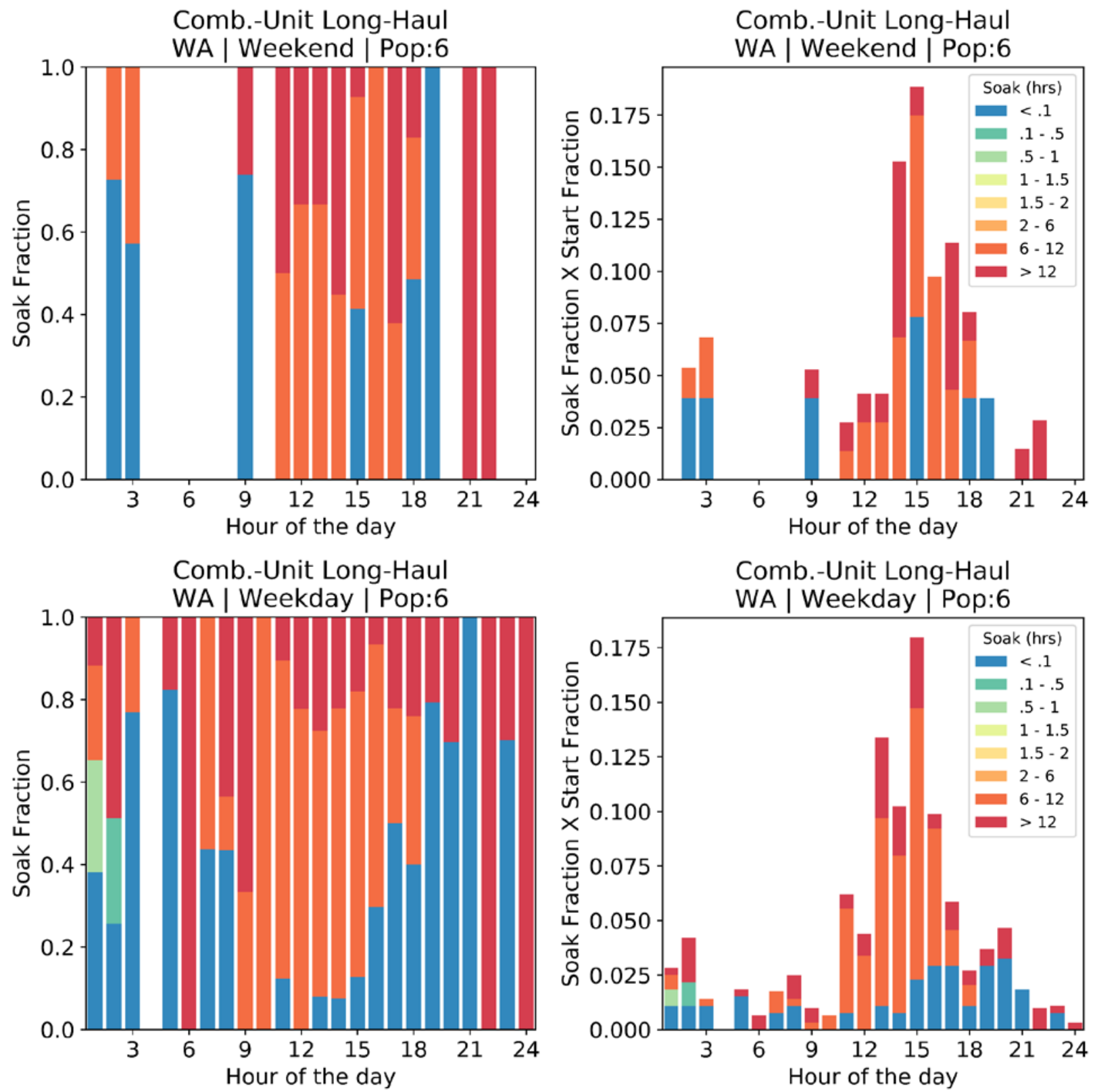

Figure H.64. Soak fraction and start fraction vs. hour of the day for Comb.-Unit Long-Haul - WA 

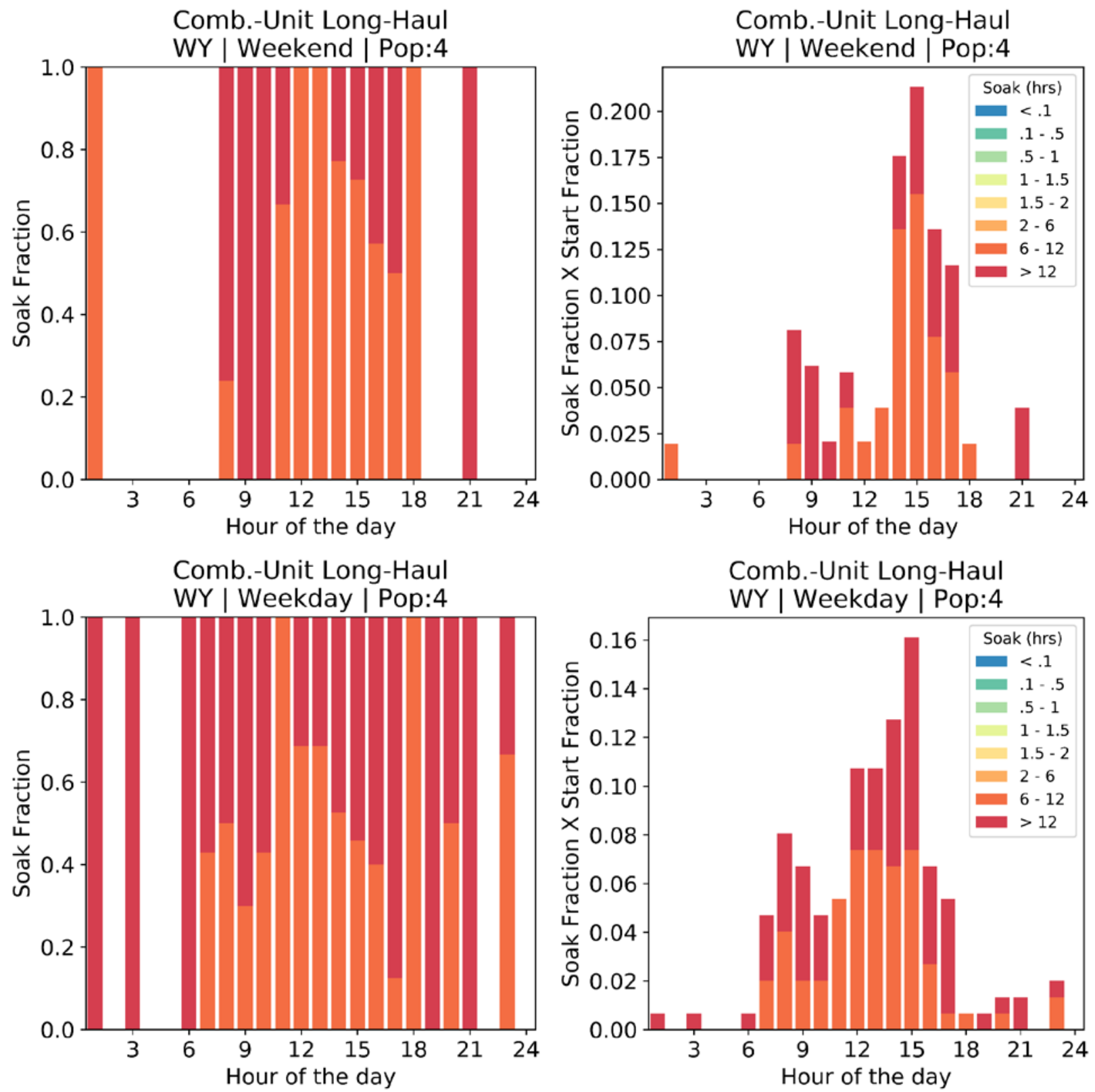

Figure H.65. Soak fraction and start fraction vs. hour of the day for Comb.-Unit Long-Haul - WY 

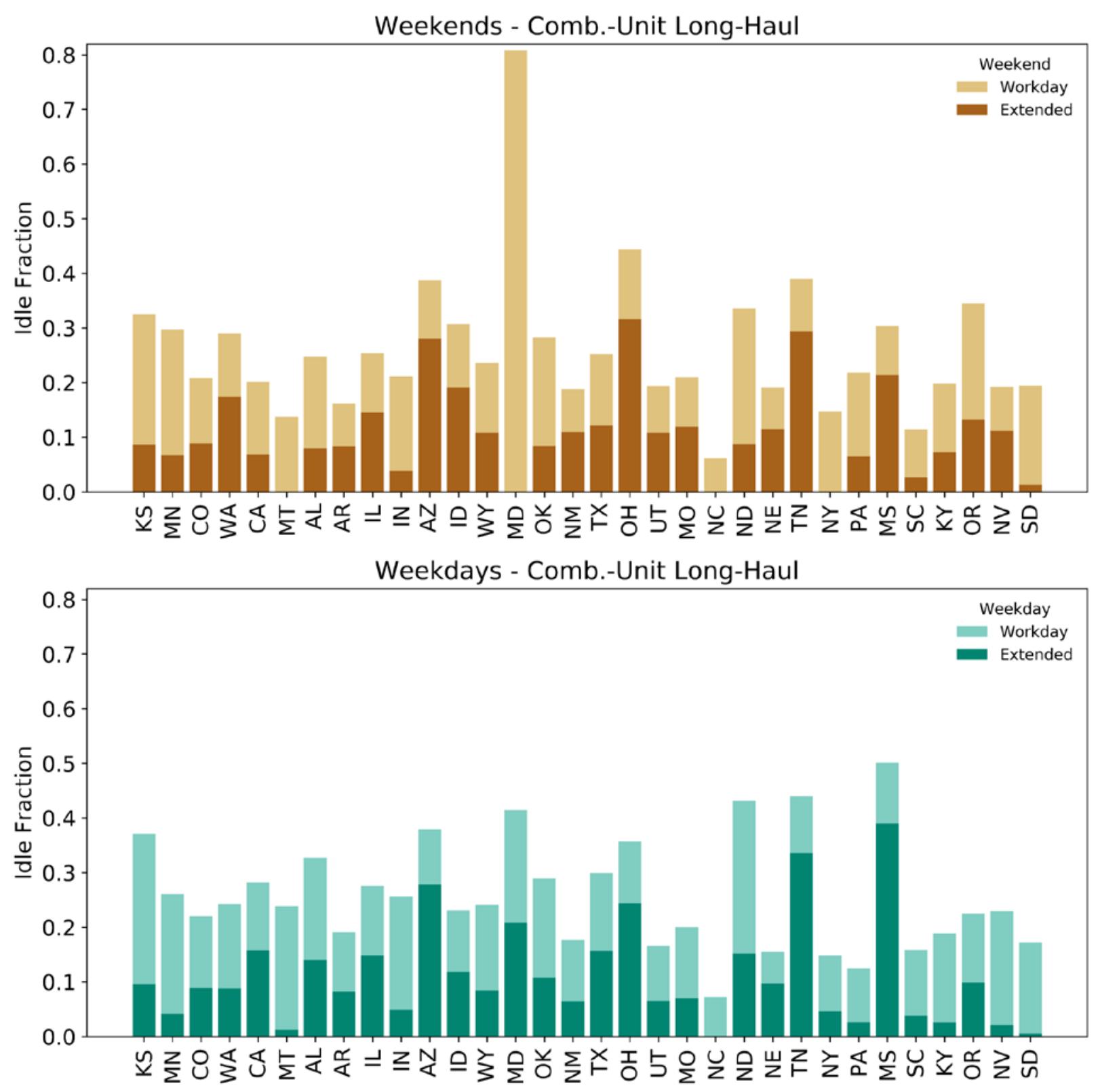

Figure H.66. Extended and workday idle fractions vs. day type and state for Comb.-Unit LongHaul. Note the Maryland idle fraction is based on a single truck and may not be representative of the state. 


\section{Appendix I: Result by State and Vocation}

\section{Single-Unit Short-Haul}
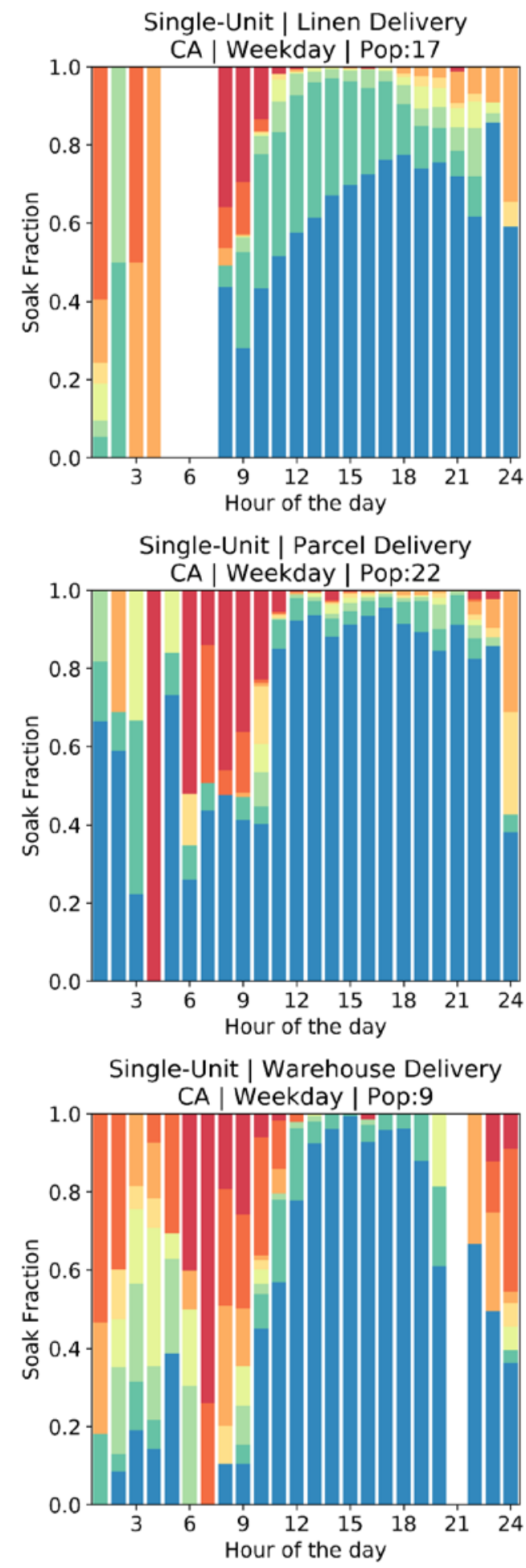

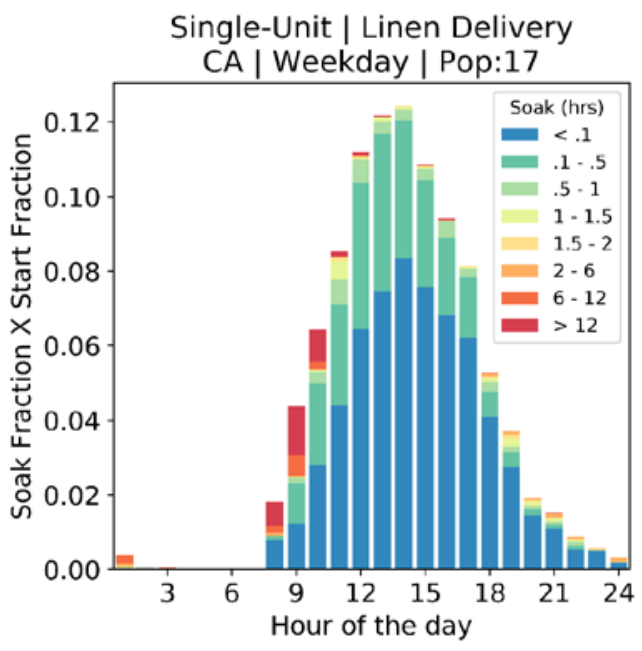

Single-Unit | Parcel Delivery

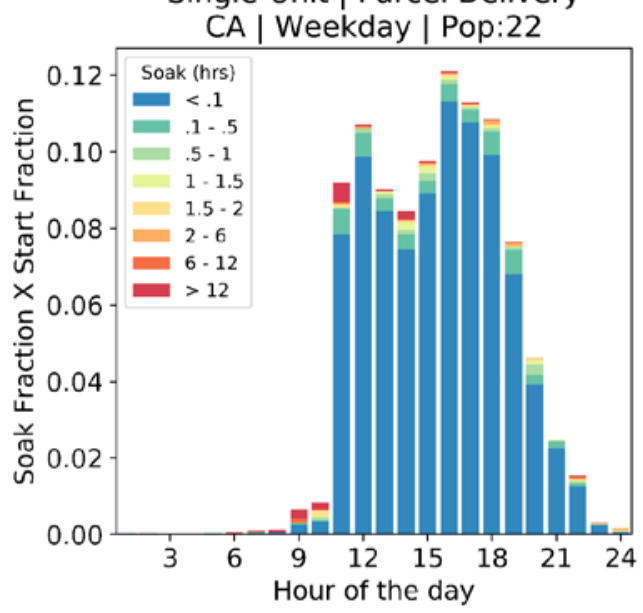

Single-Unit | Warehouse Delivery CA | Weekday | Pop:9

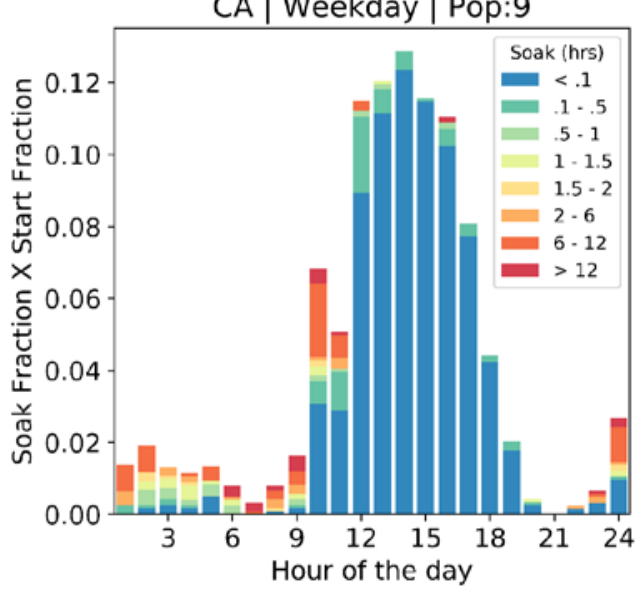



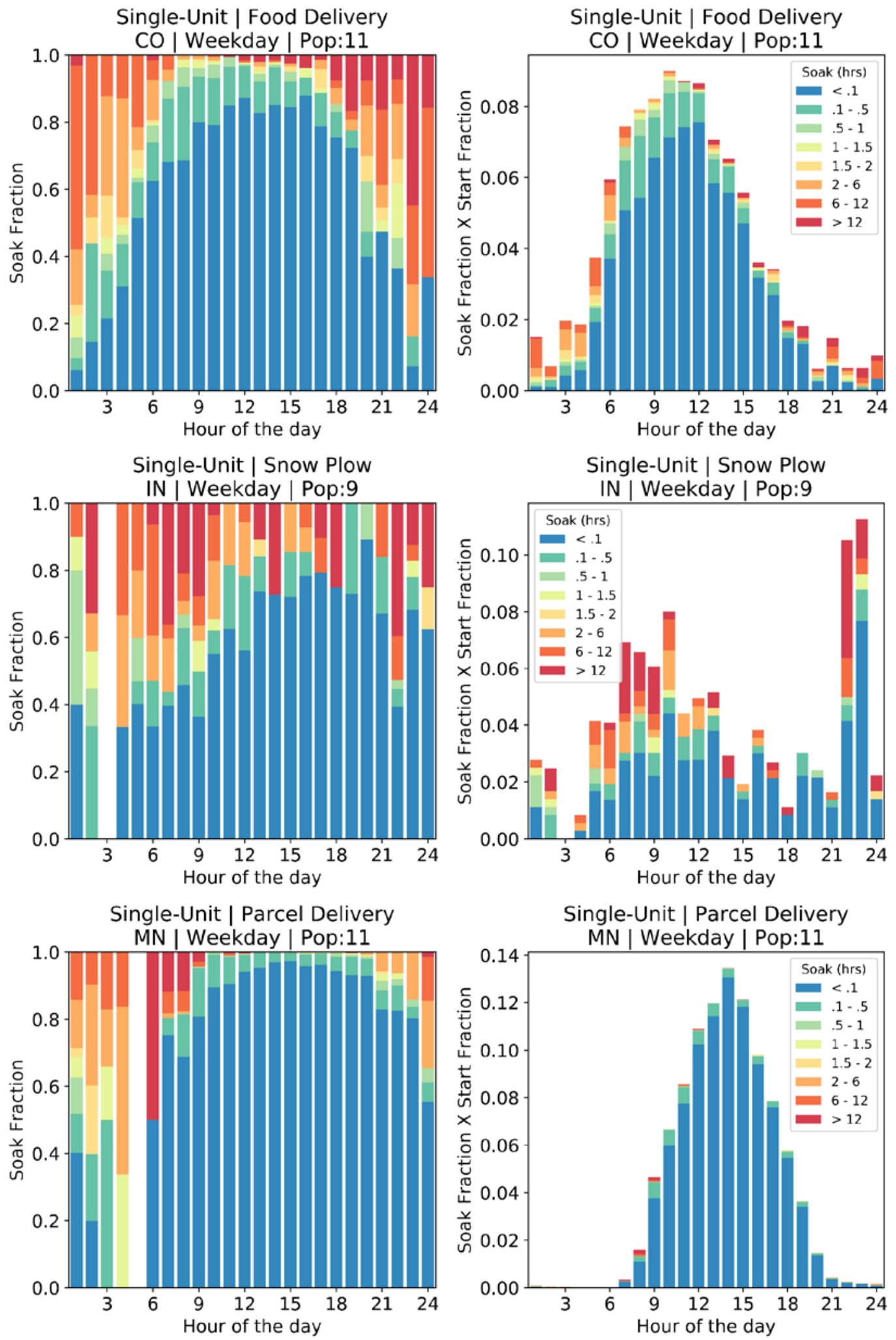


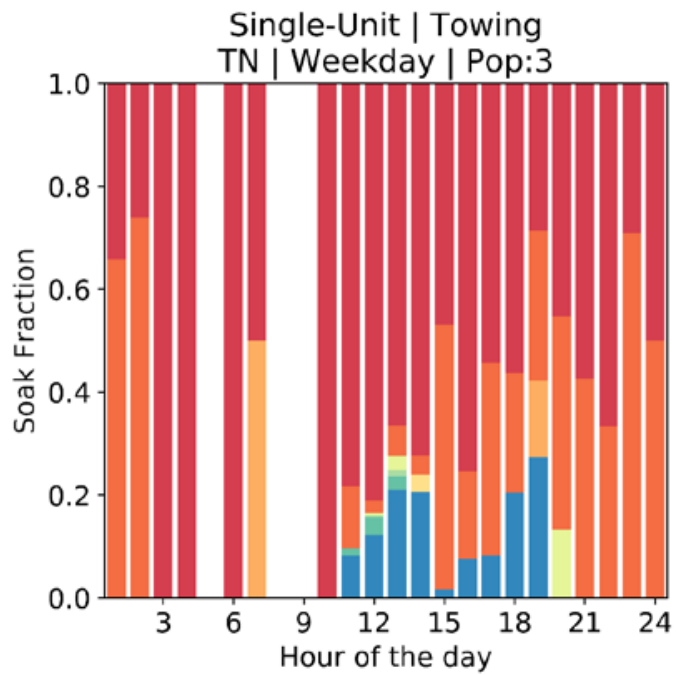

Single-Unit | Concrete
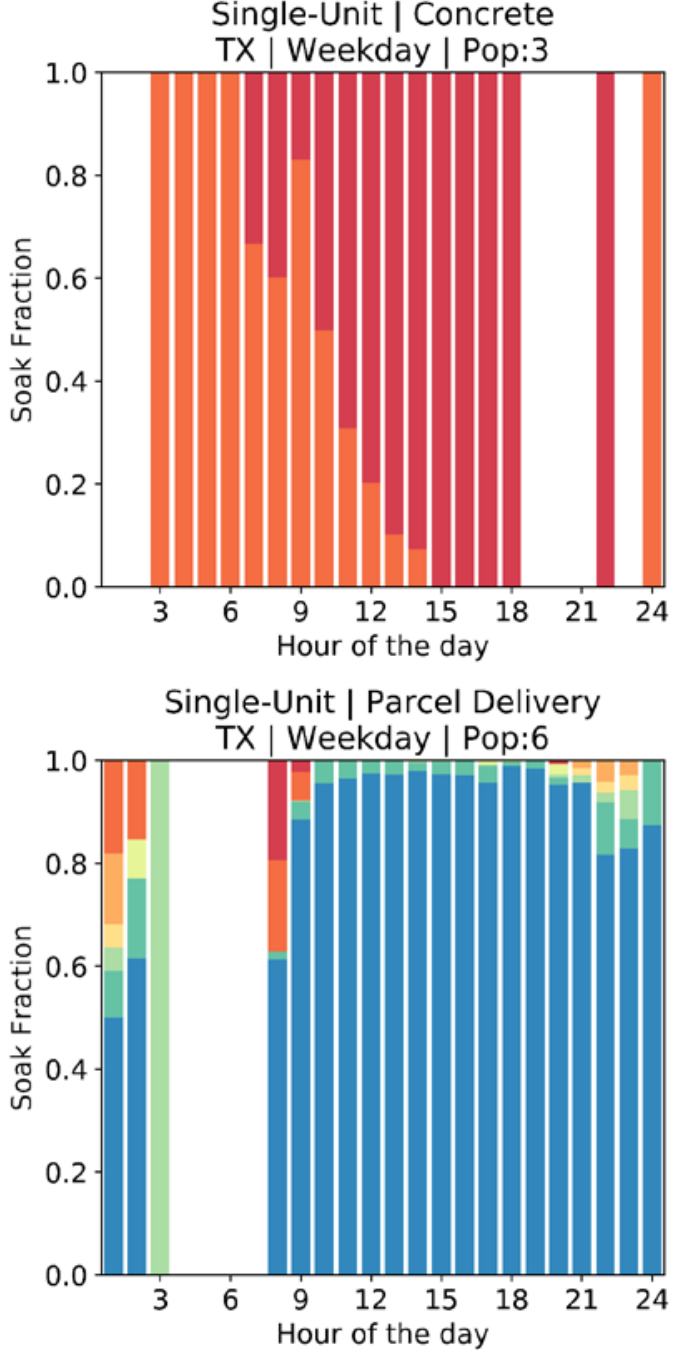

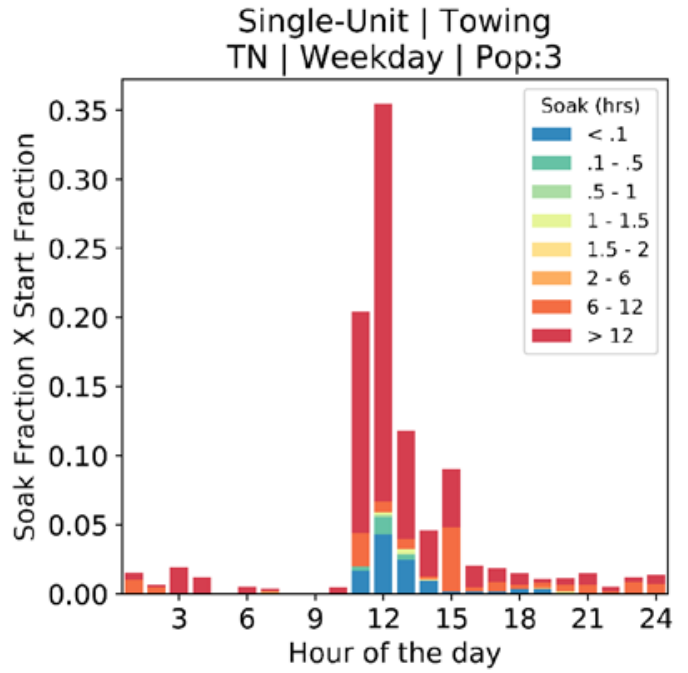

Single-Unit | Concrete TX | Weekday | Pop:3

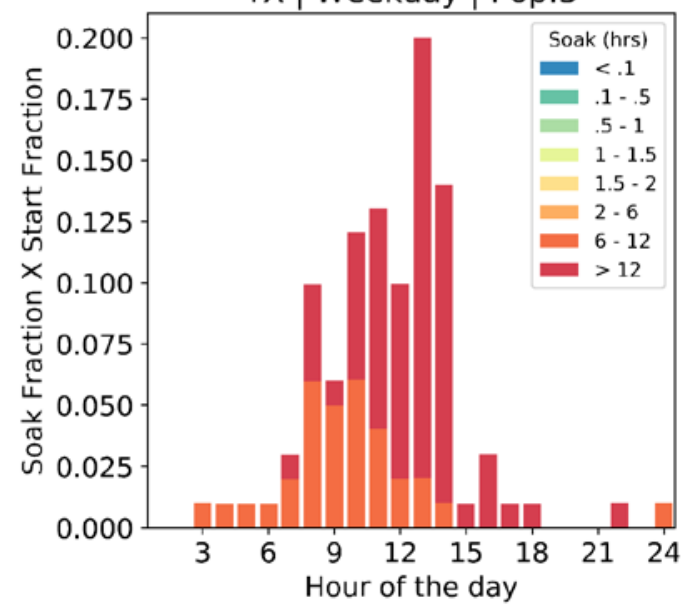

Single-Unit | Parcel Delivery

TX | Weekday | Pop:6

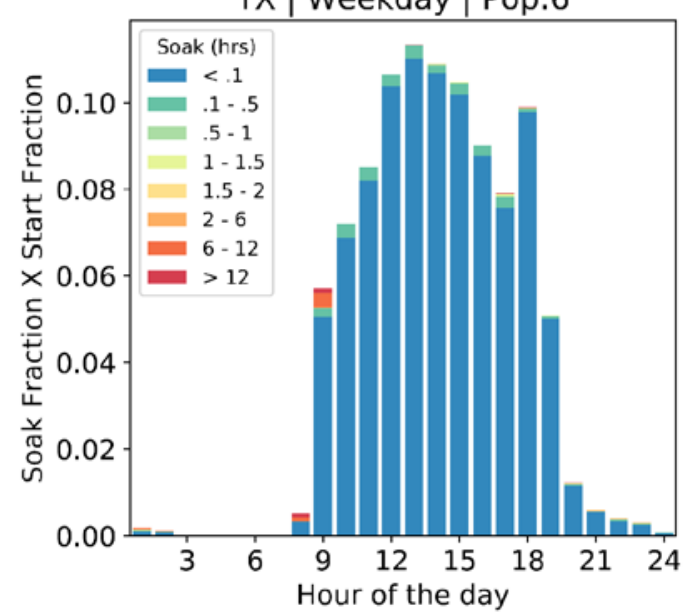



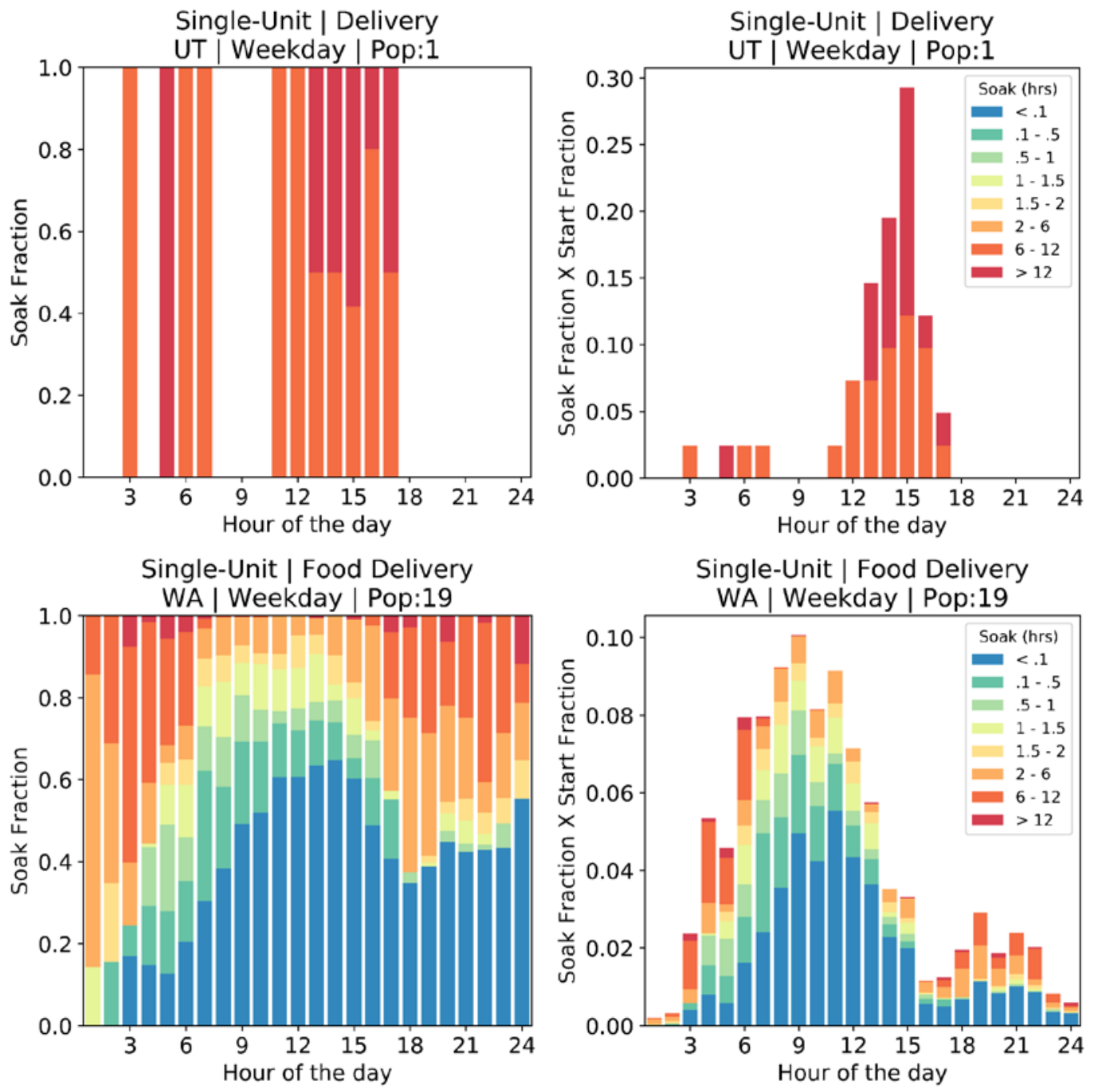


\section{Combination-Unit Short-Haul}
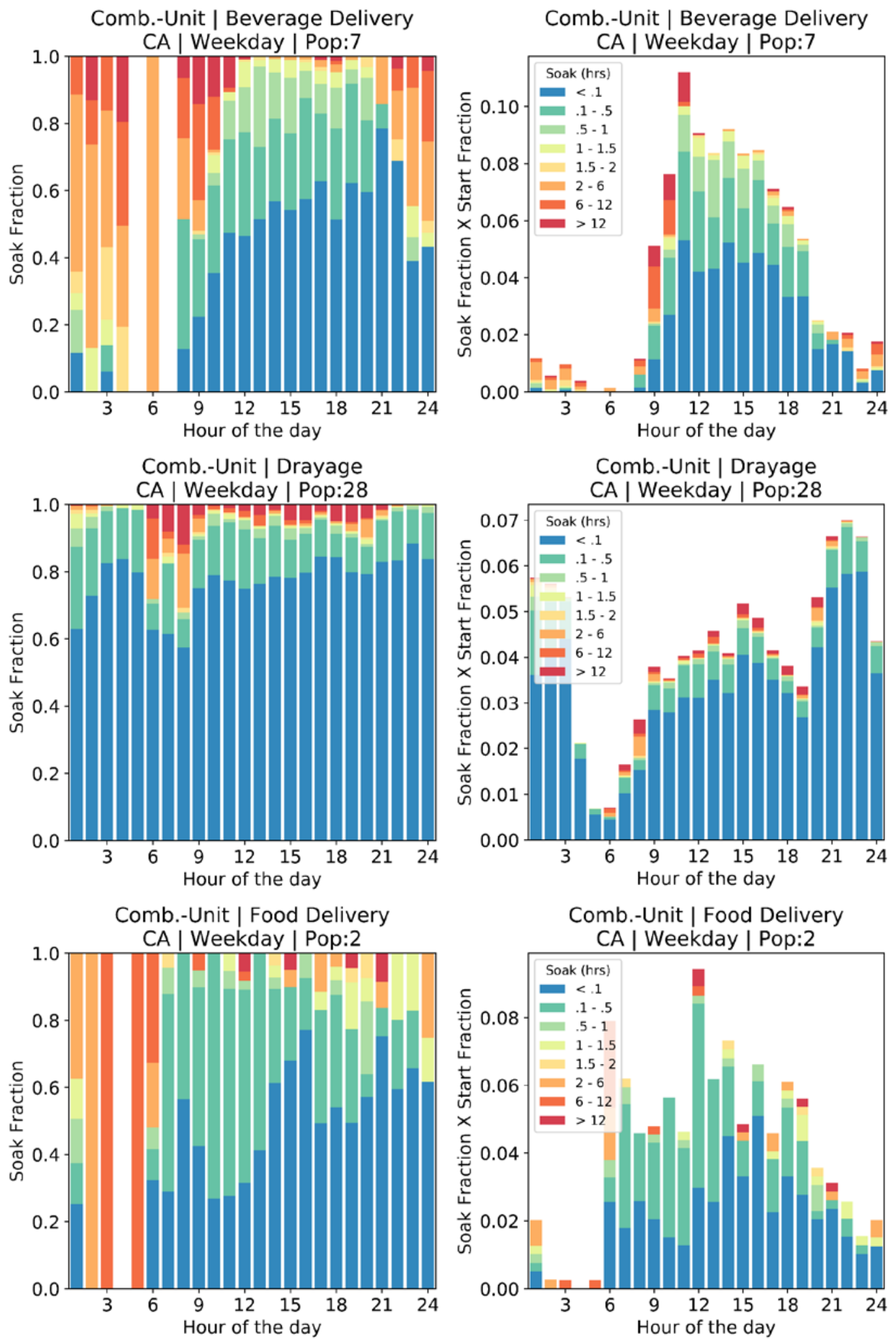

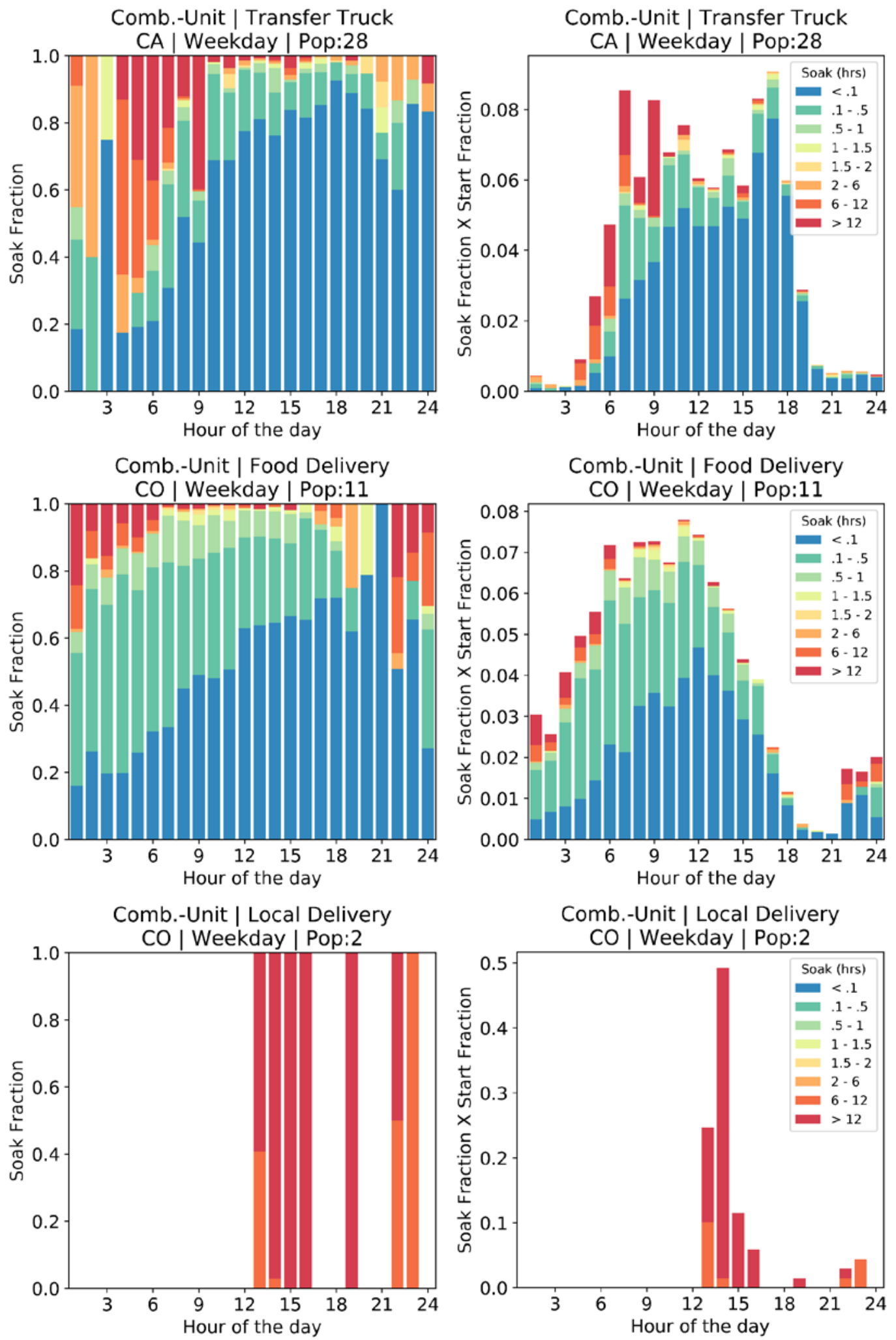

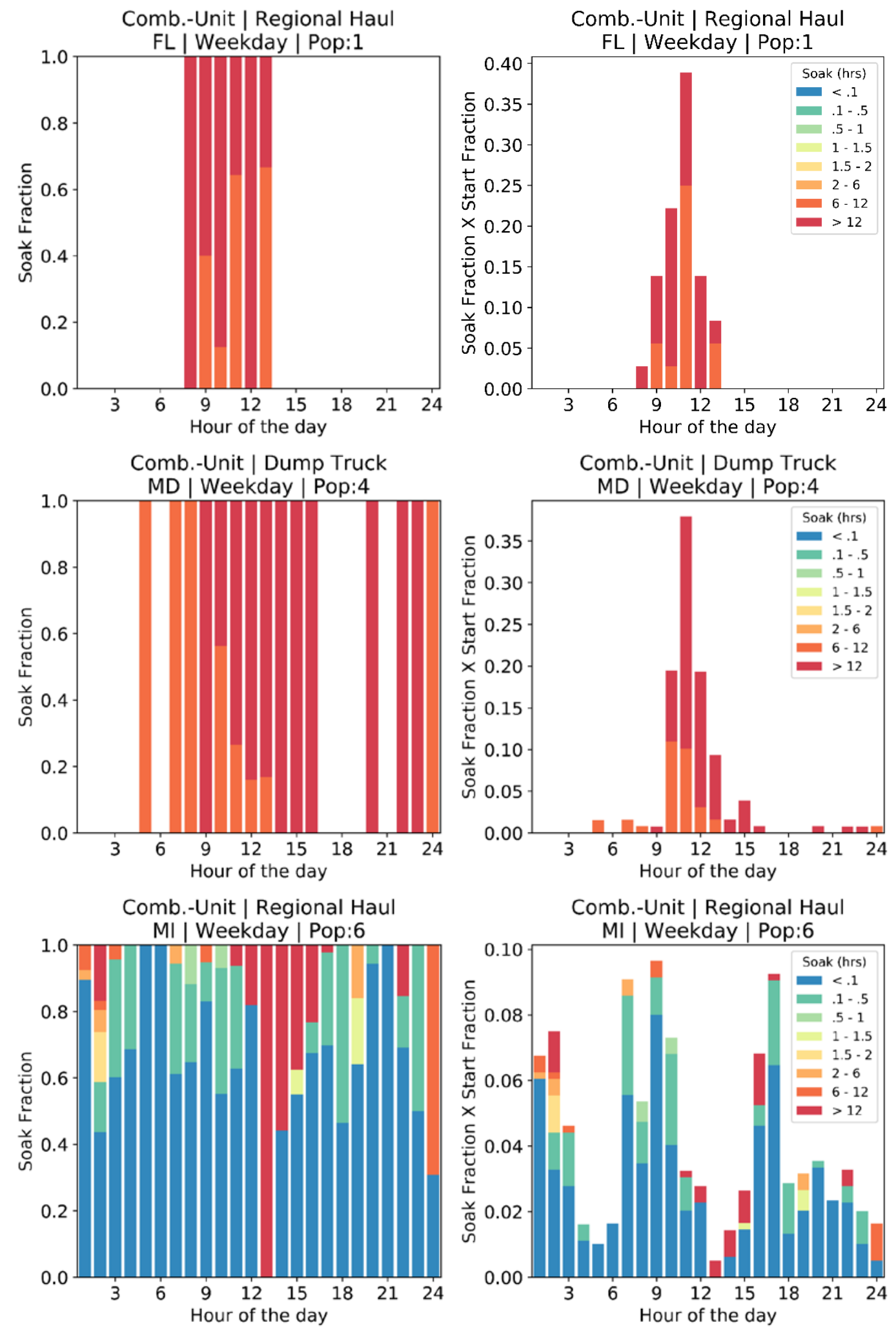

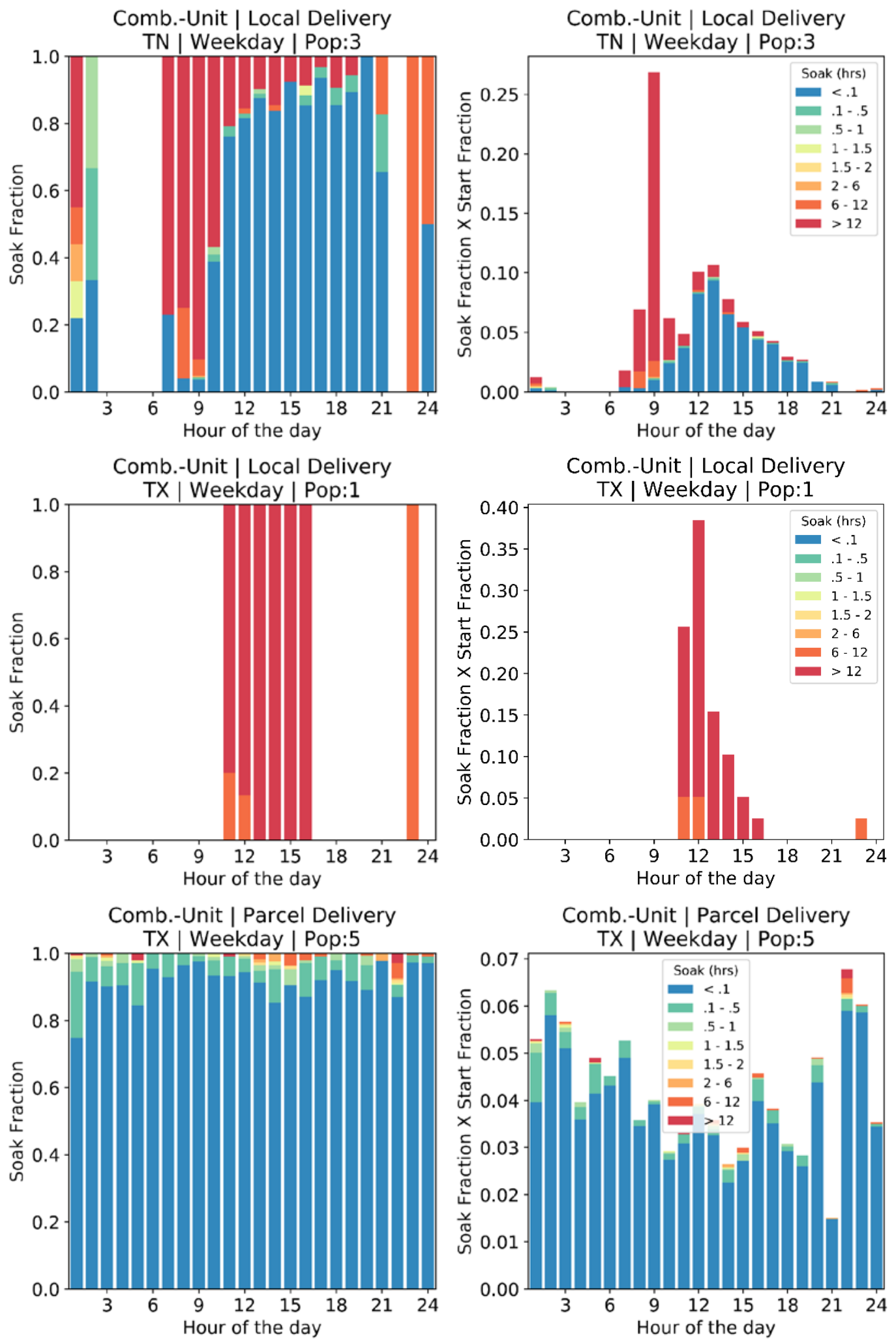

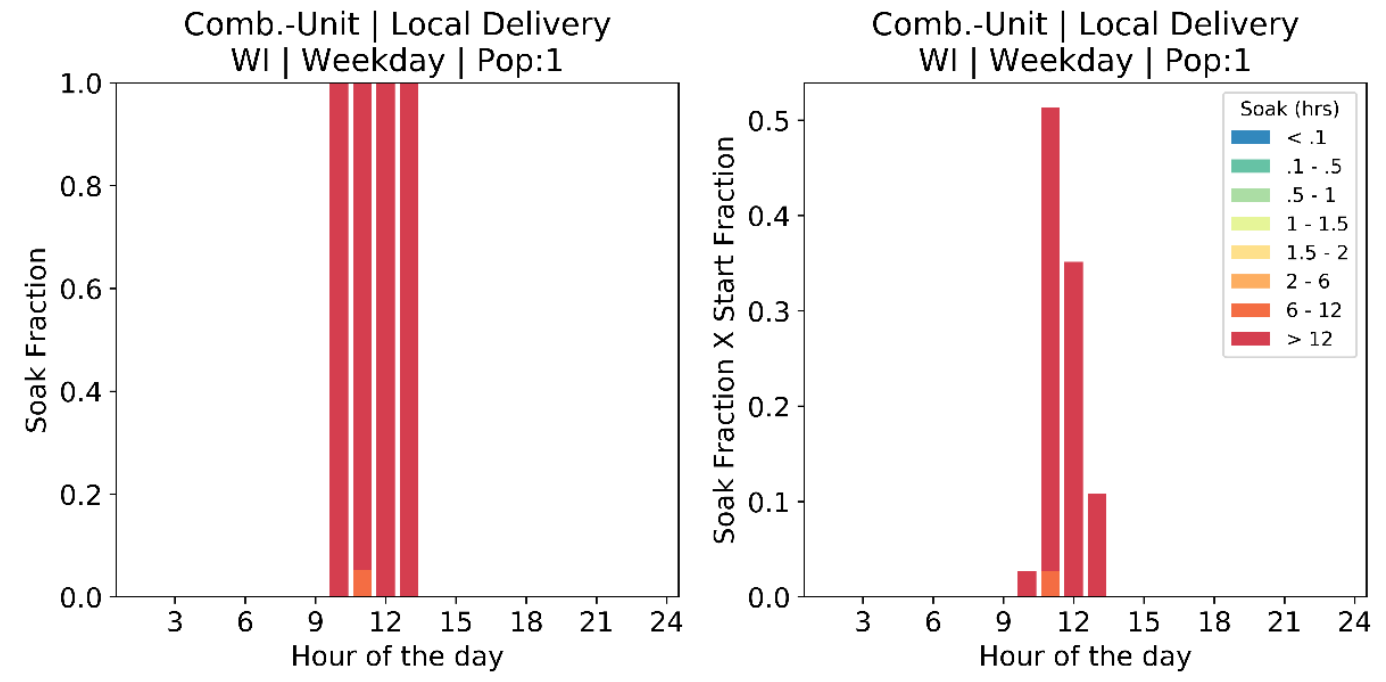

This report is available at no cost from the National Renewable Energy Laboratory (NREL) at www.nrel.gov/publications. 


\section{Appendix J: Result by Vocation}
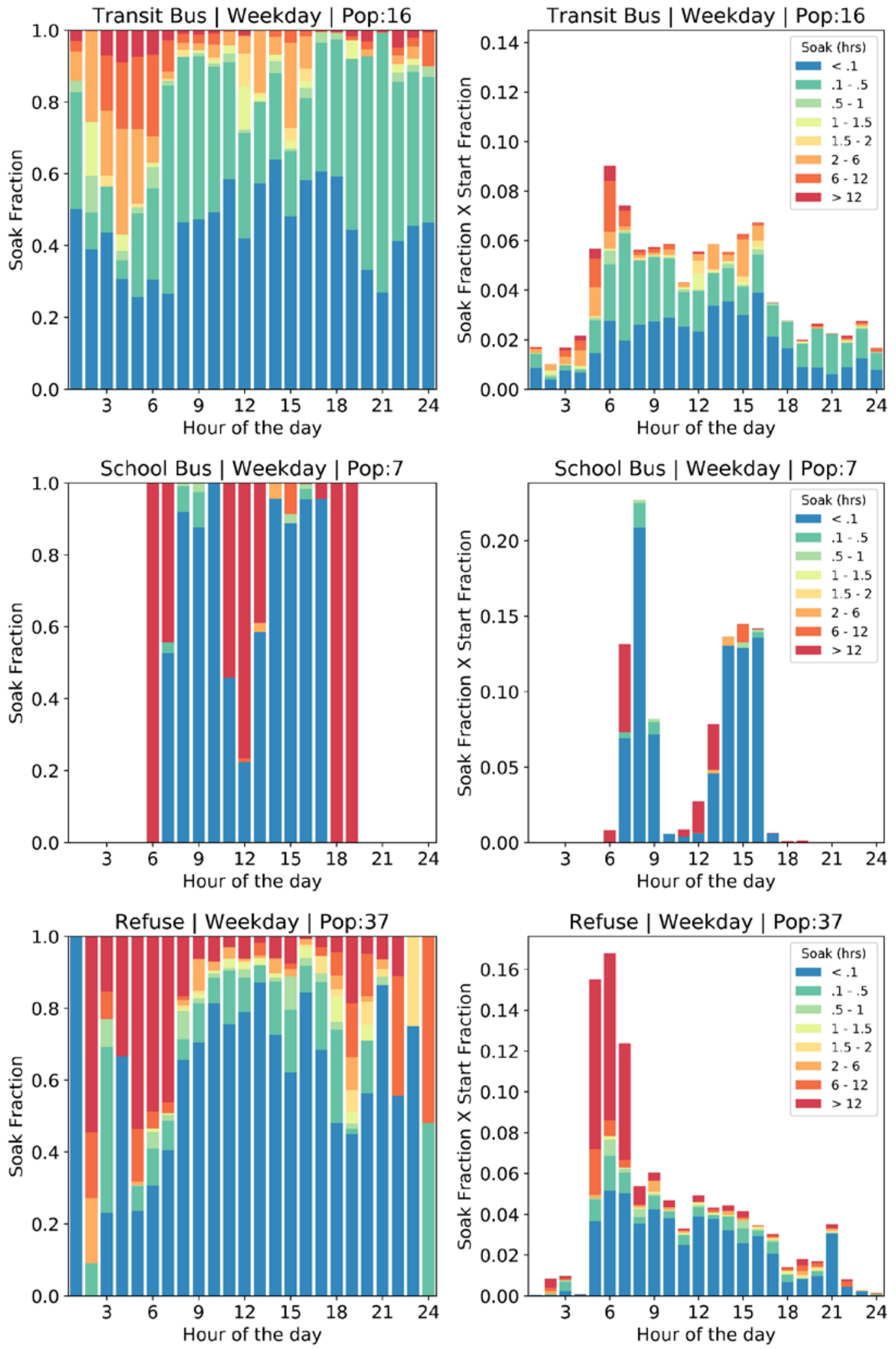

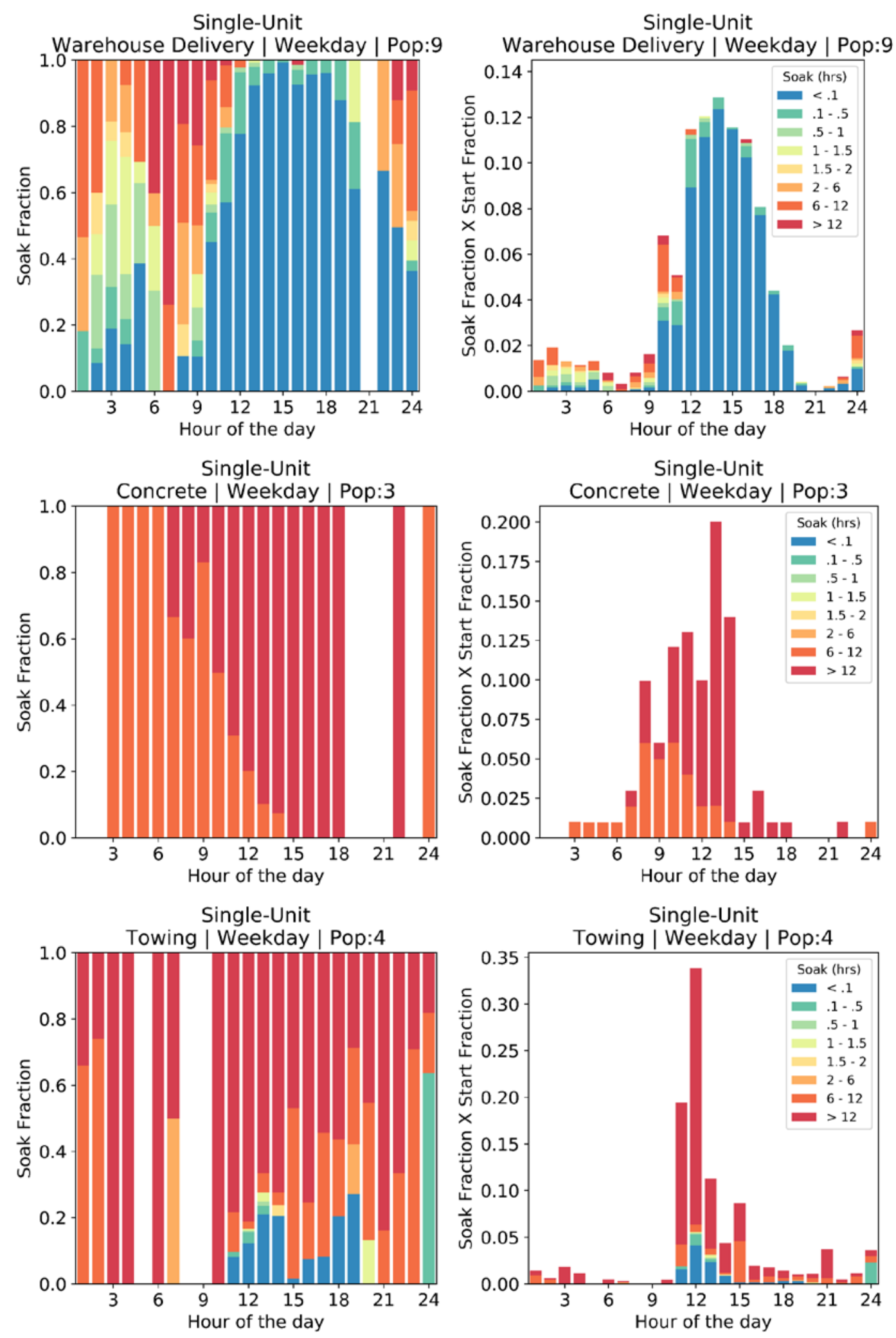

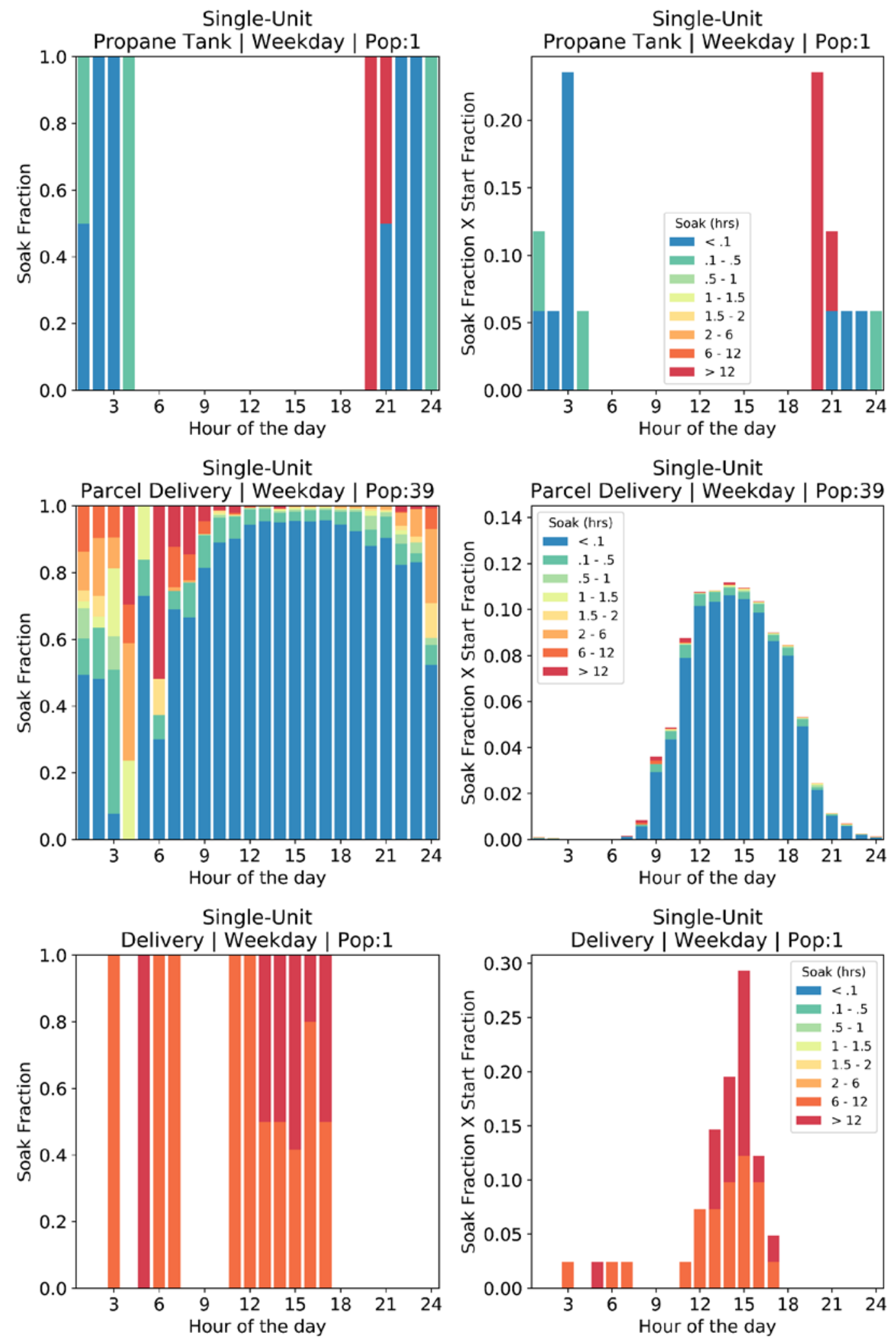

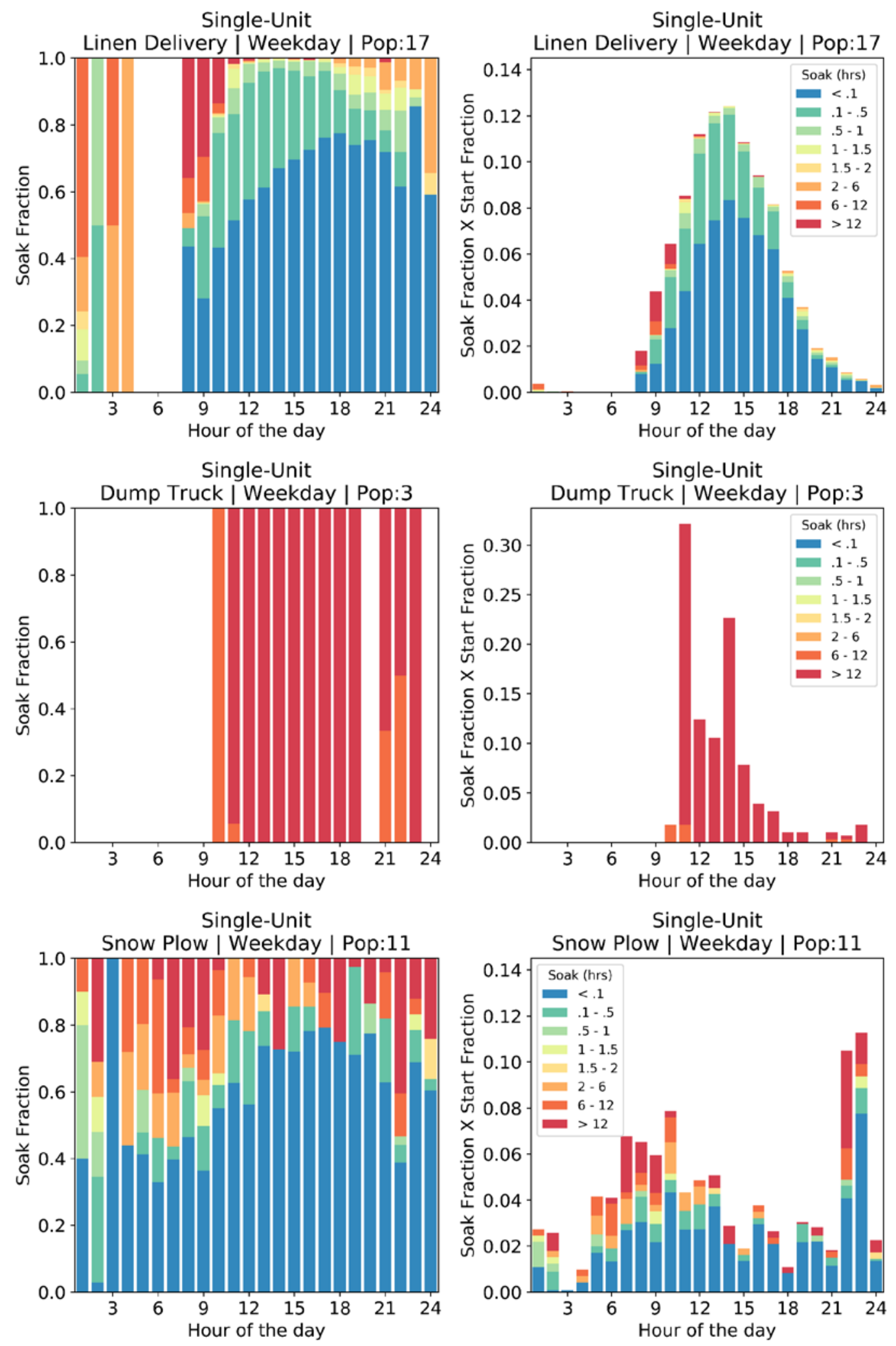

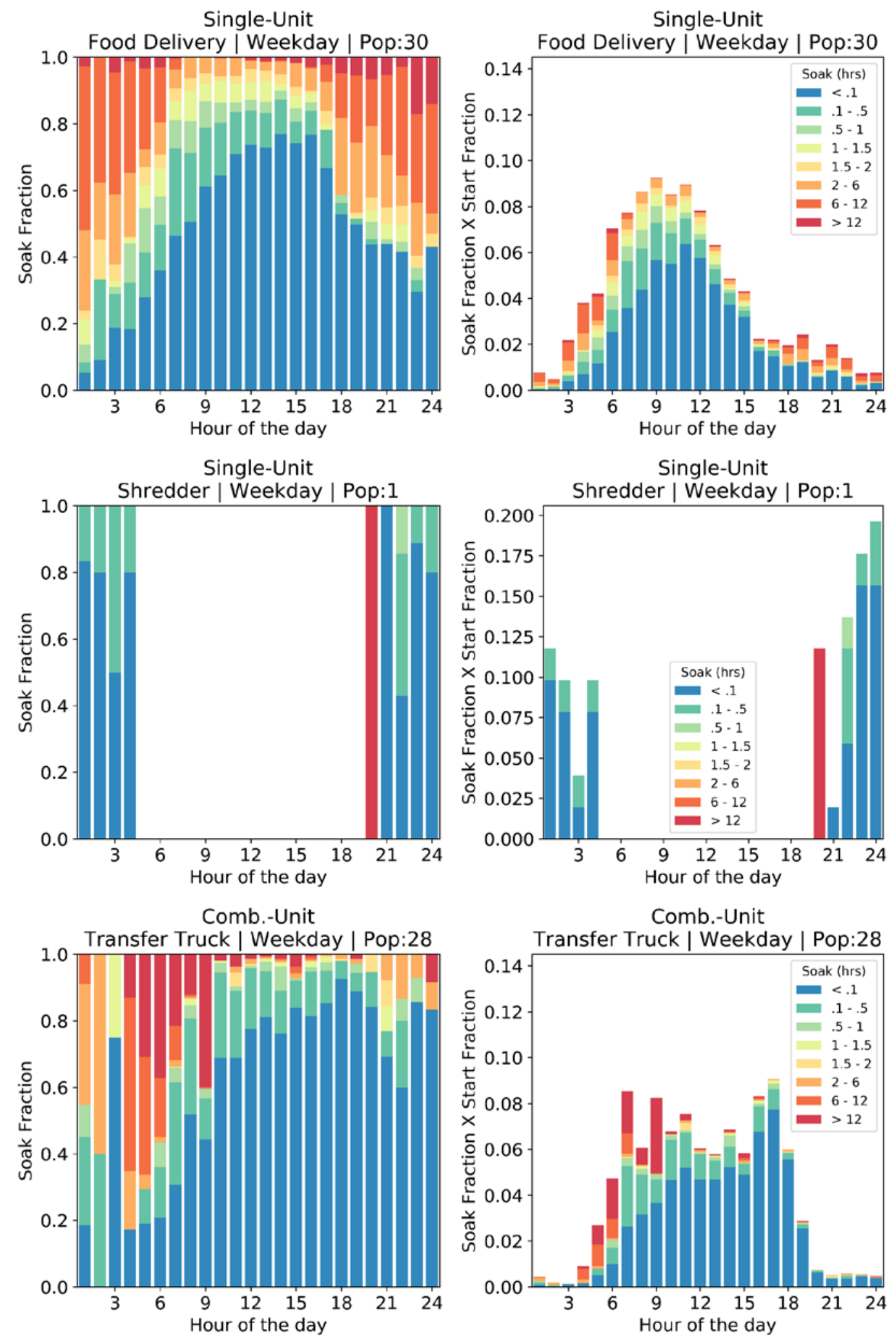

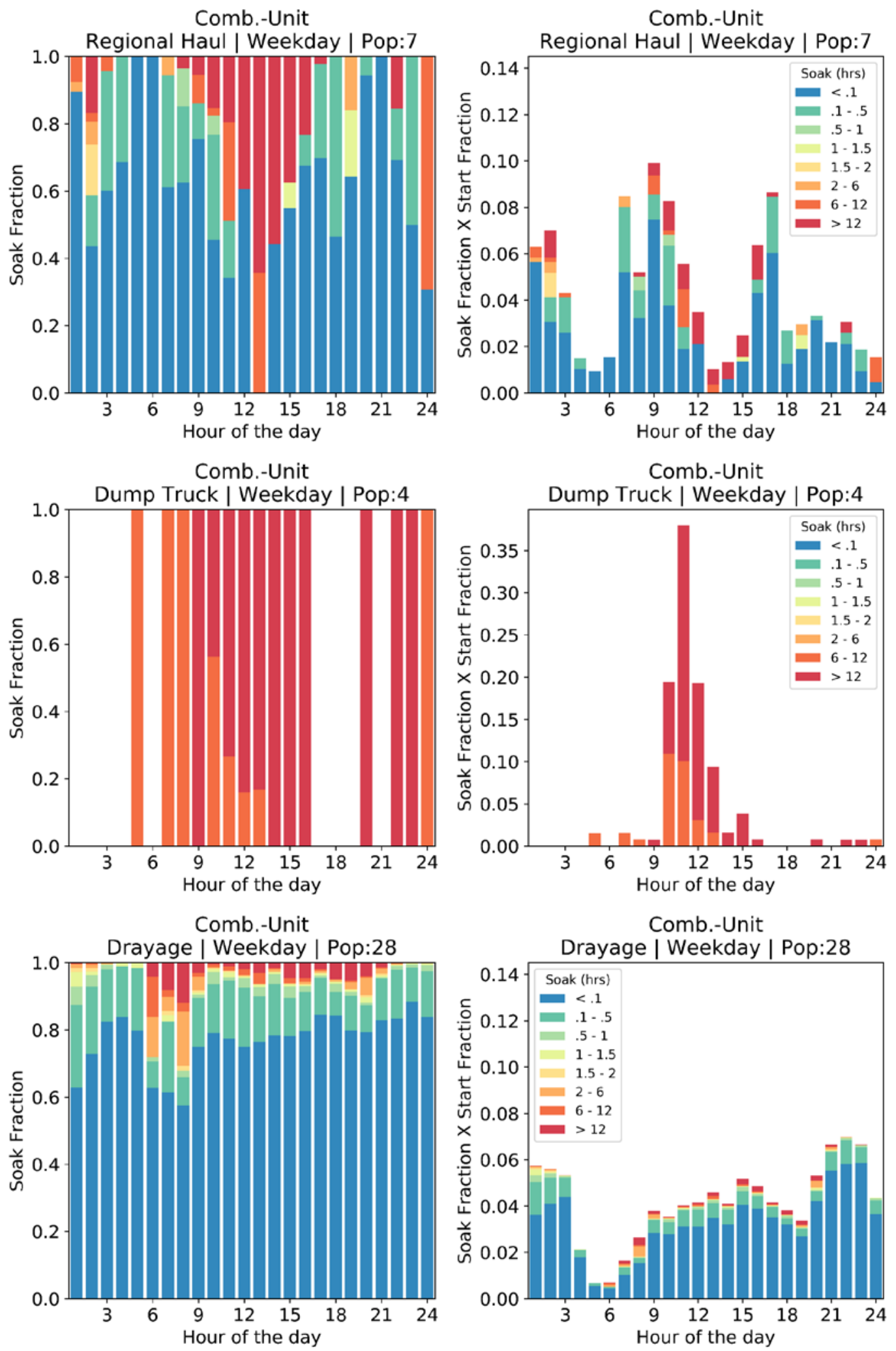

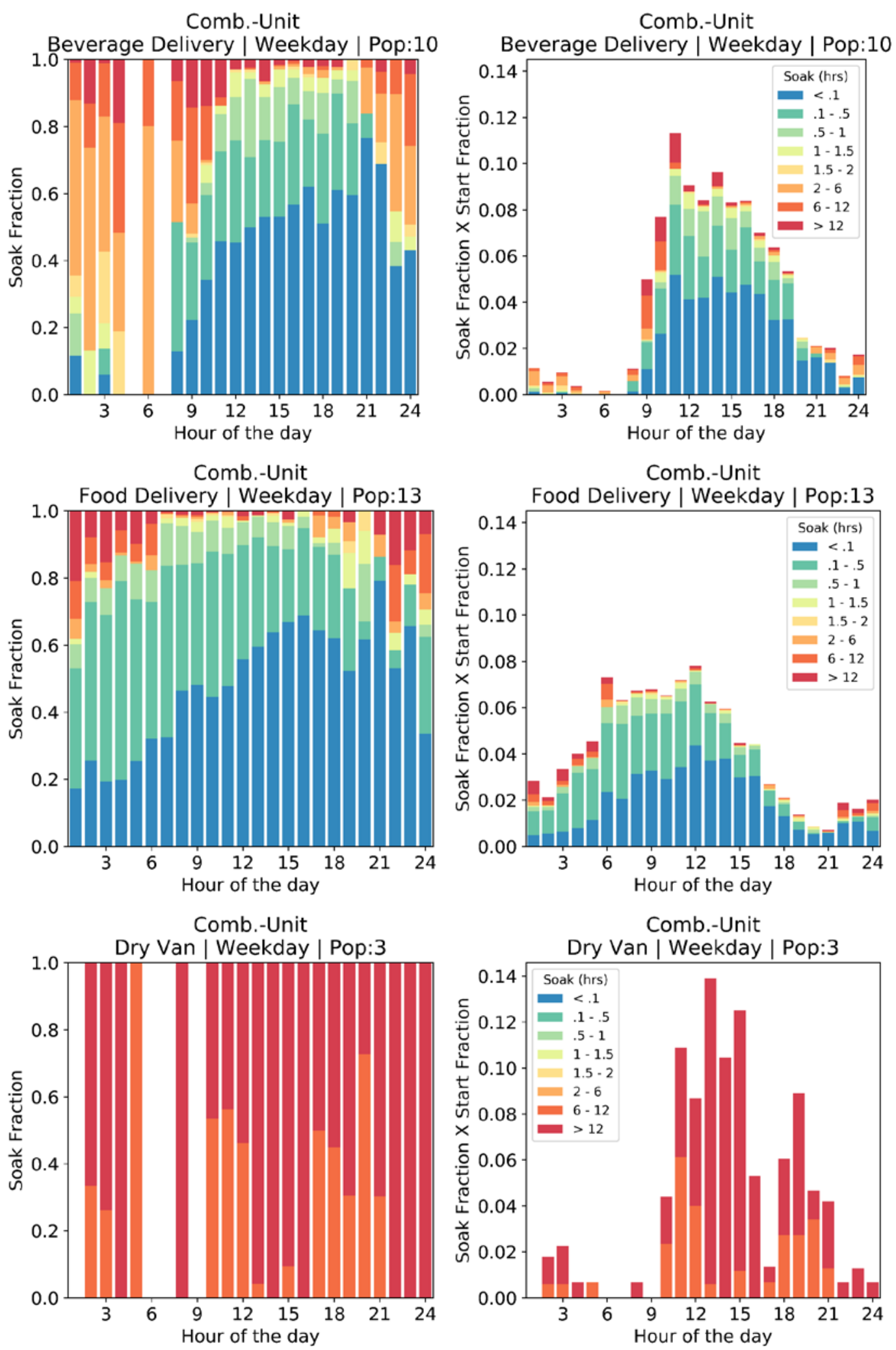

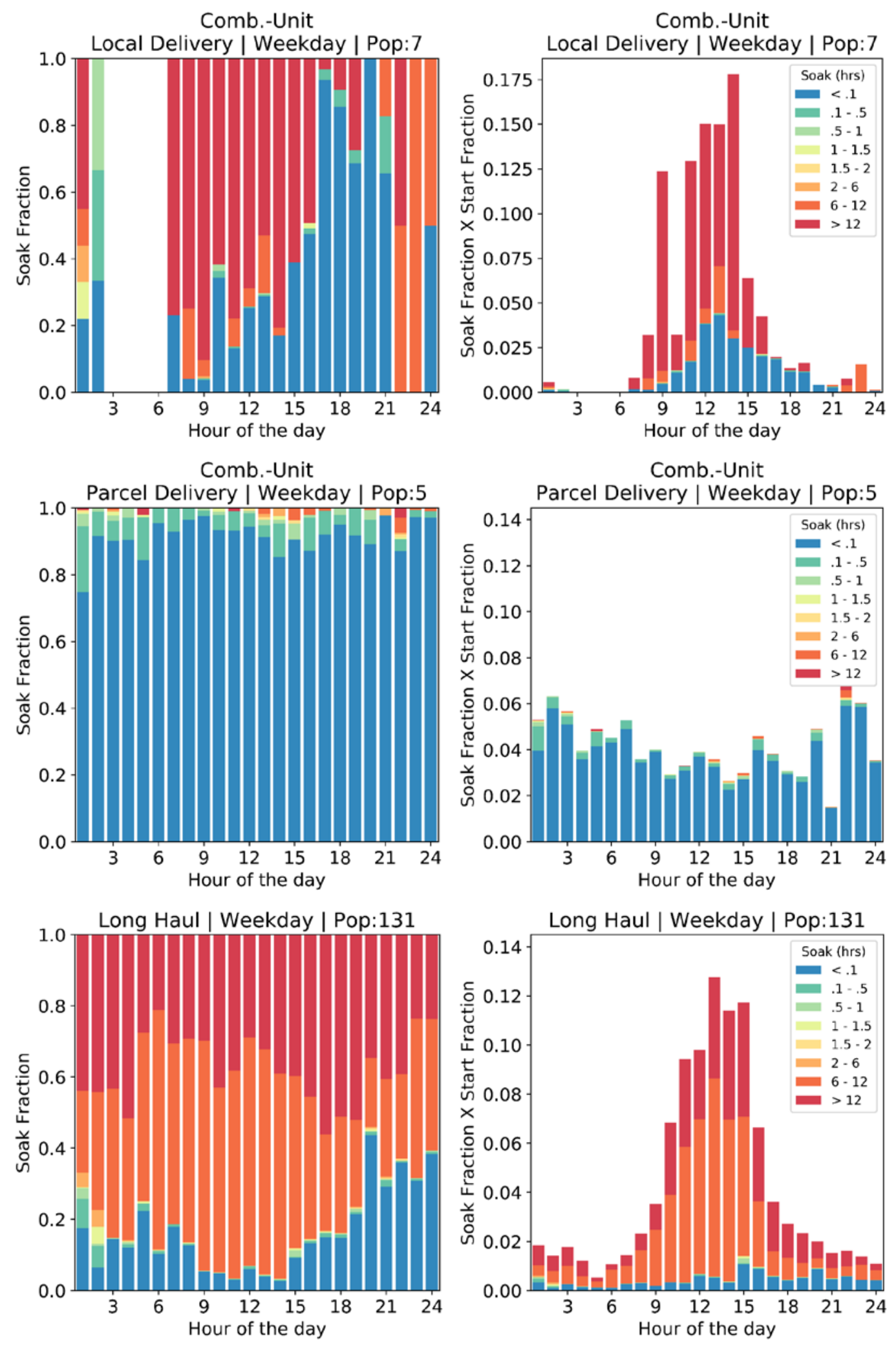
Note: CU indicates Combination-Unit and SU indicates Single-Unit for short haul vehicles
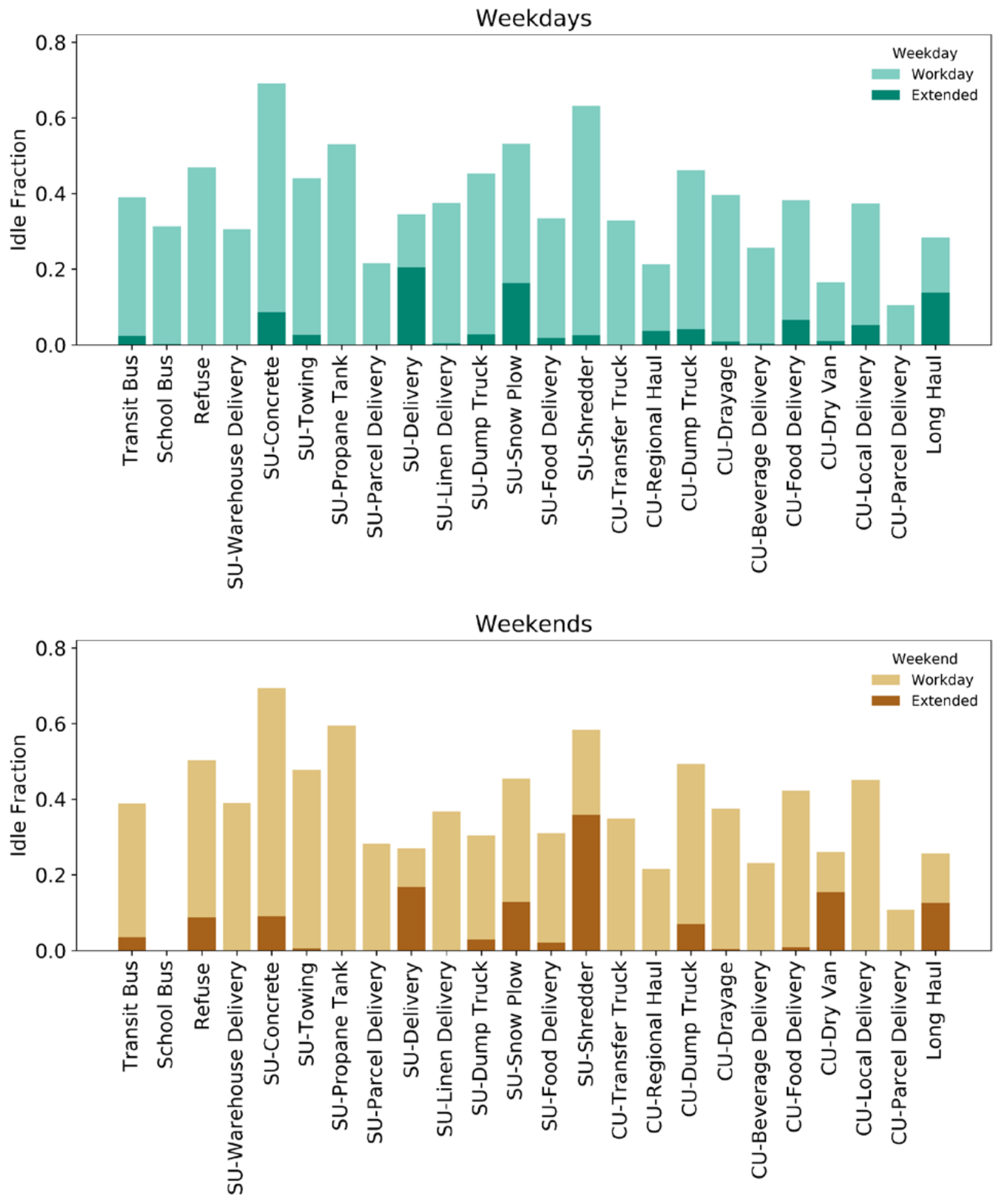


\section{Appendix K: Method Comparison}

\section{Soak times Start Fraction Comparison \\ Old}
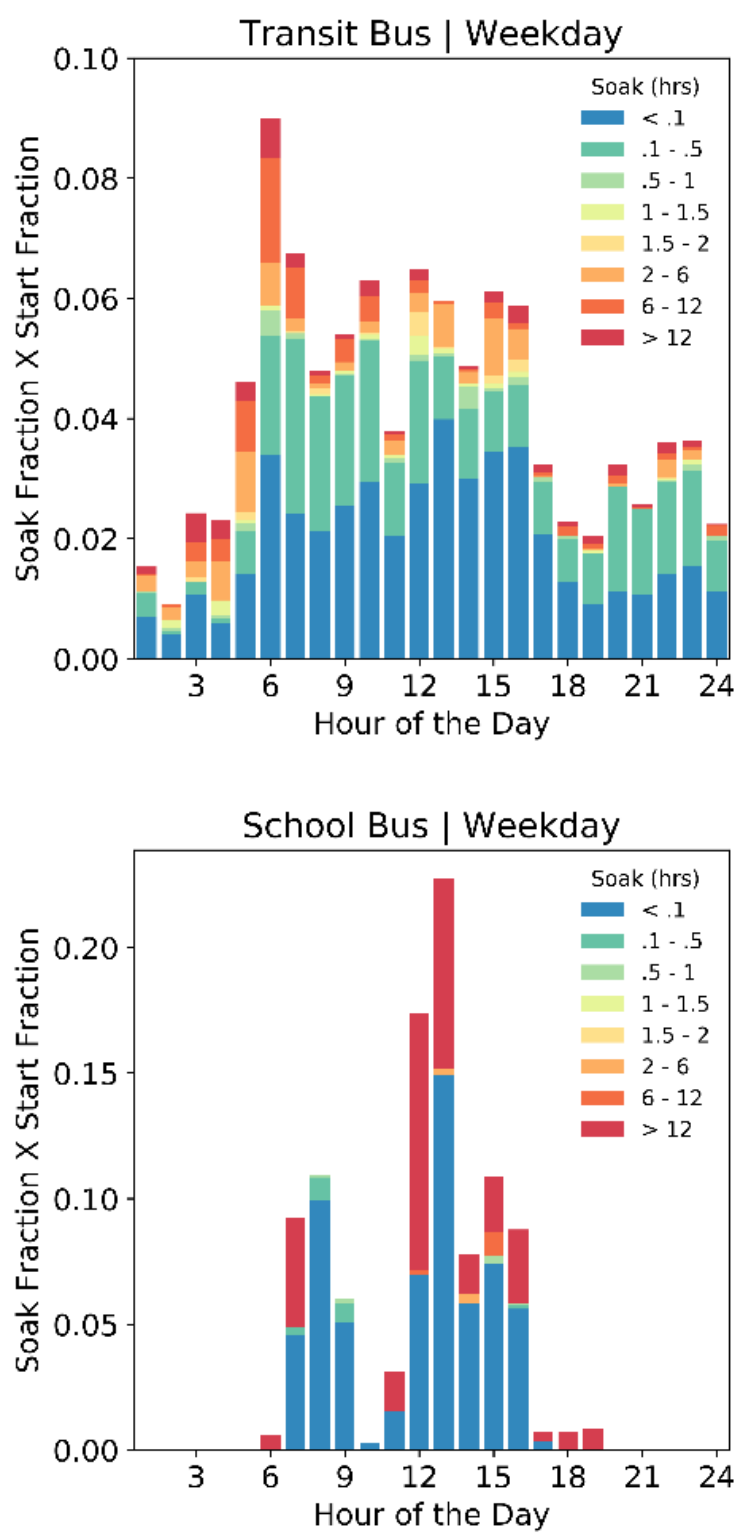

New
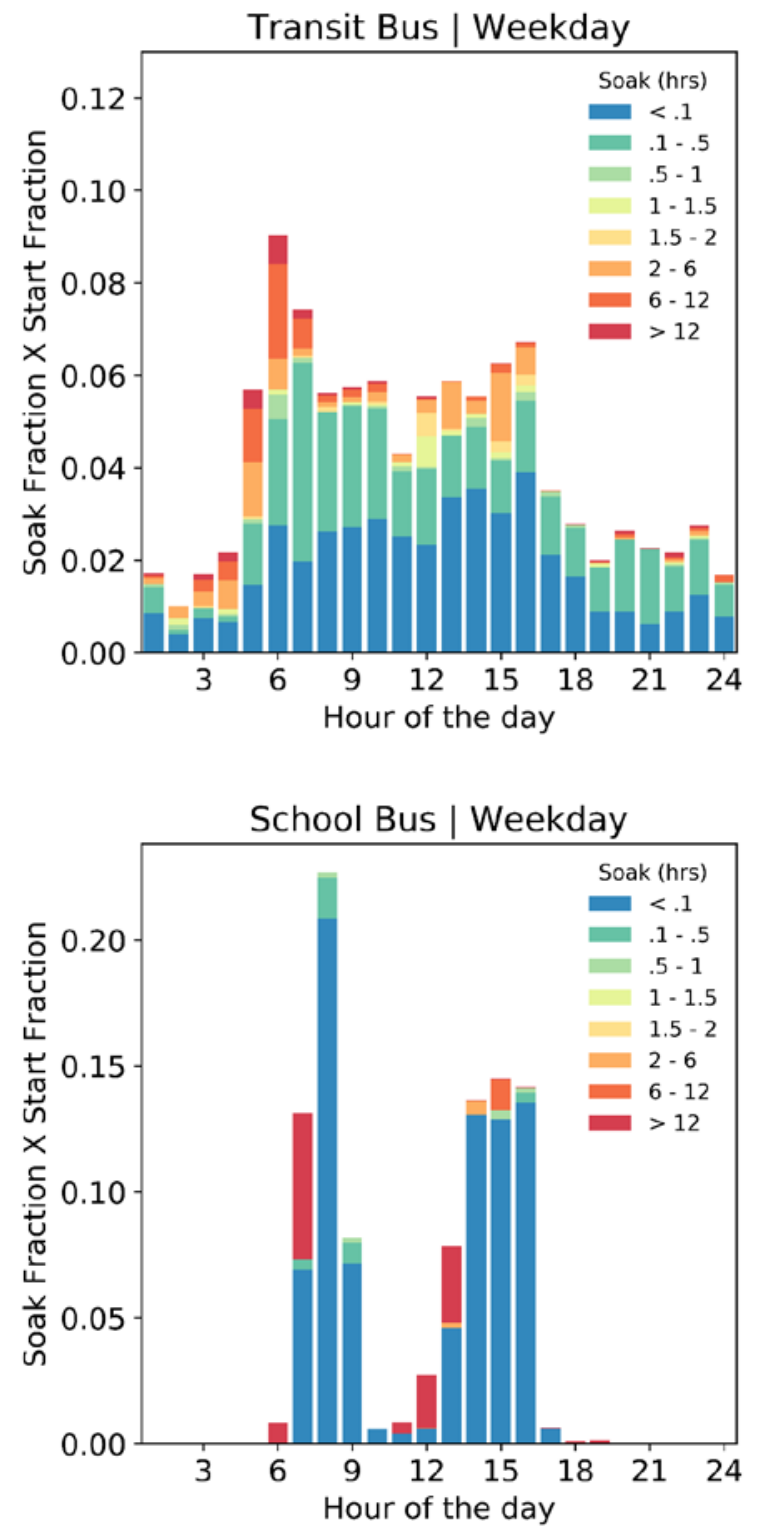
Old
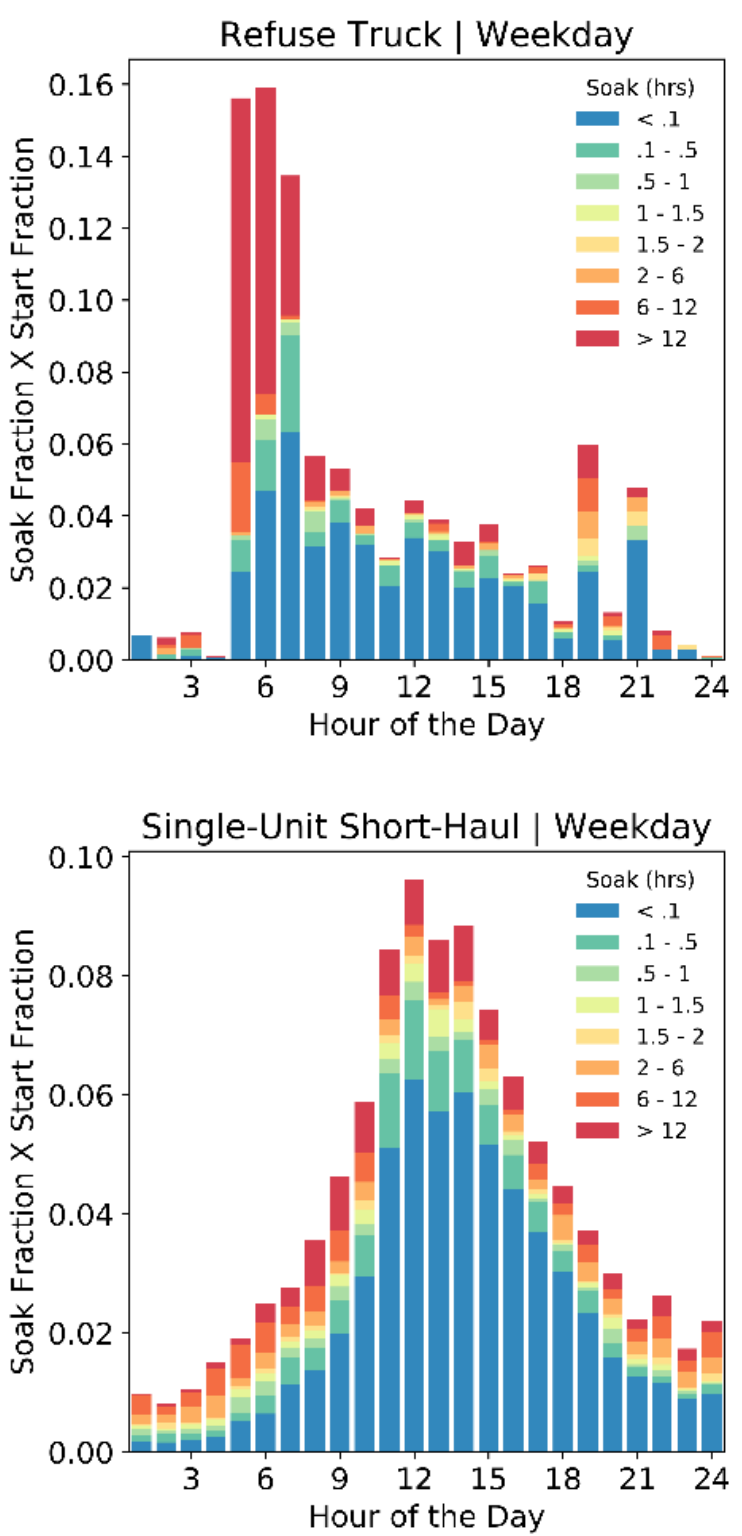

New
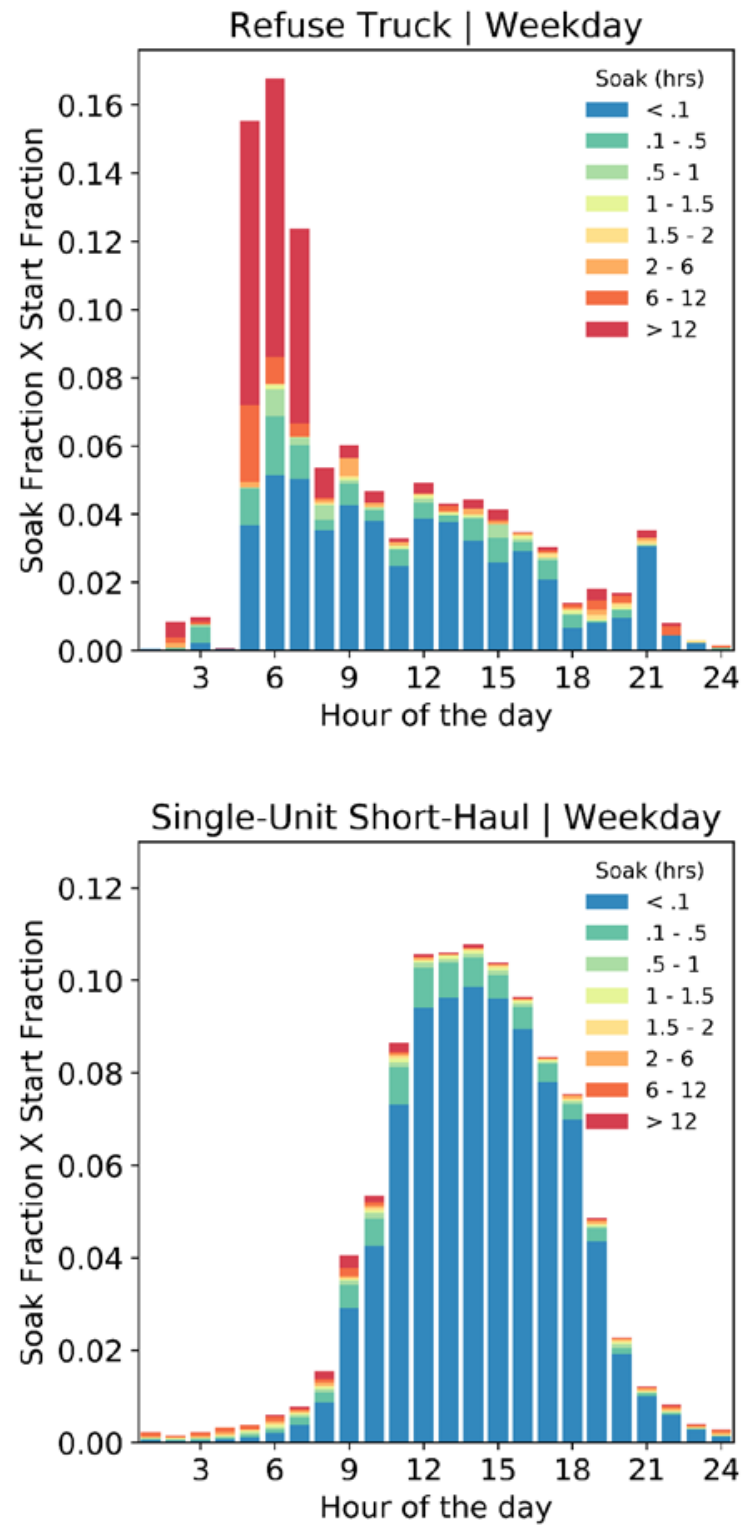

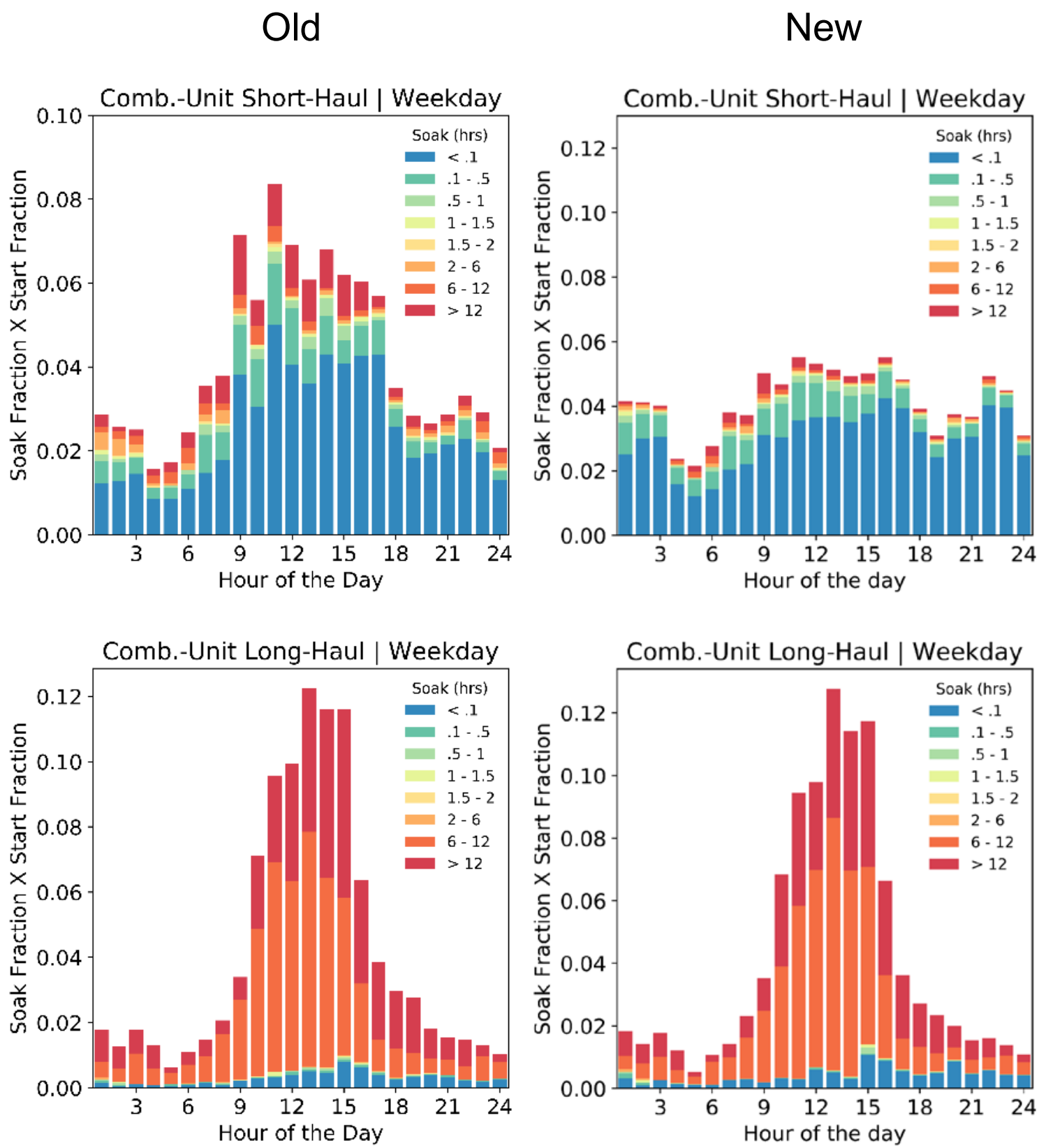


\section{Idle Fraction Comparison}
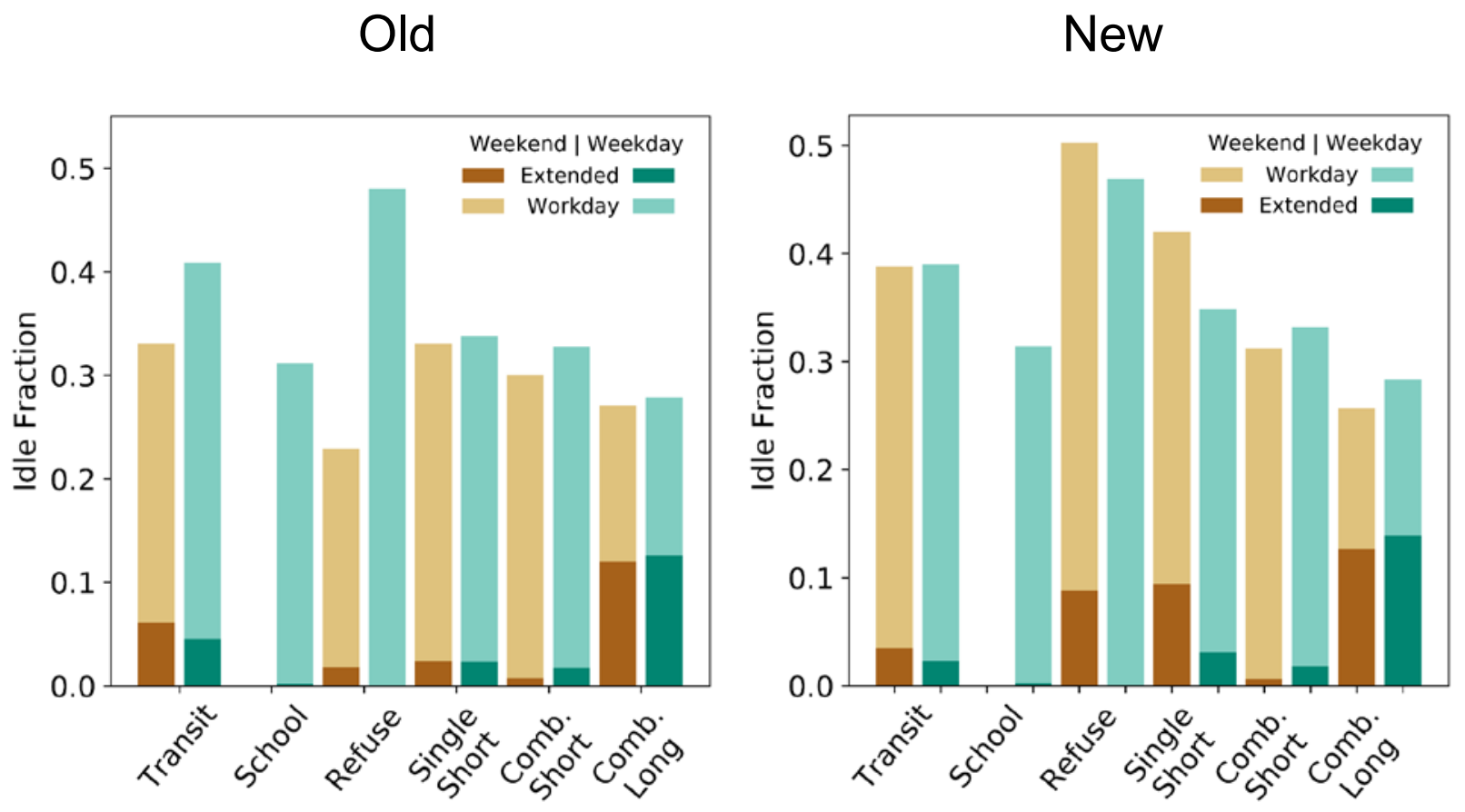
Old
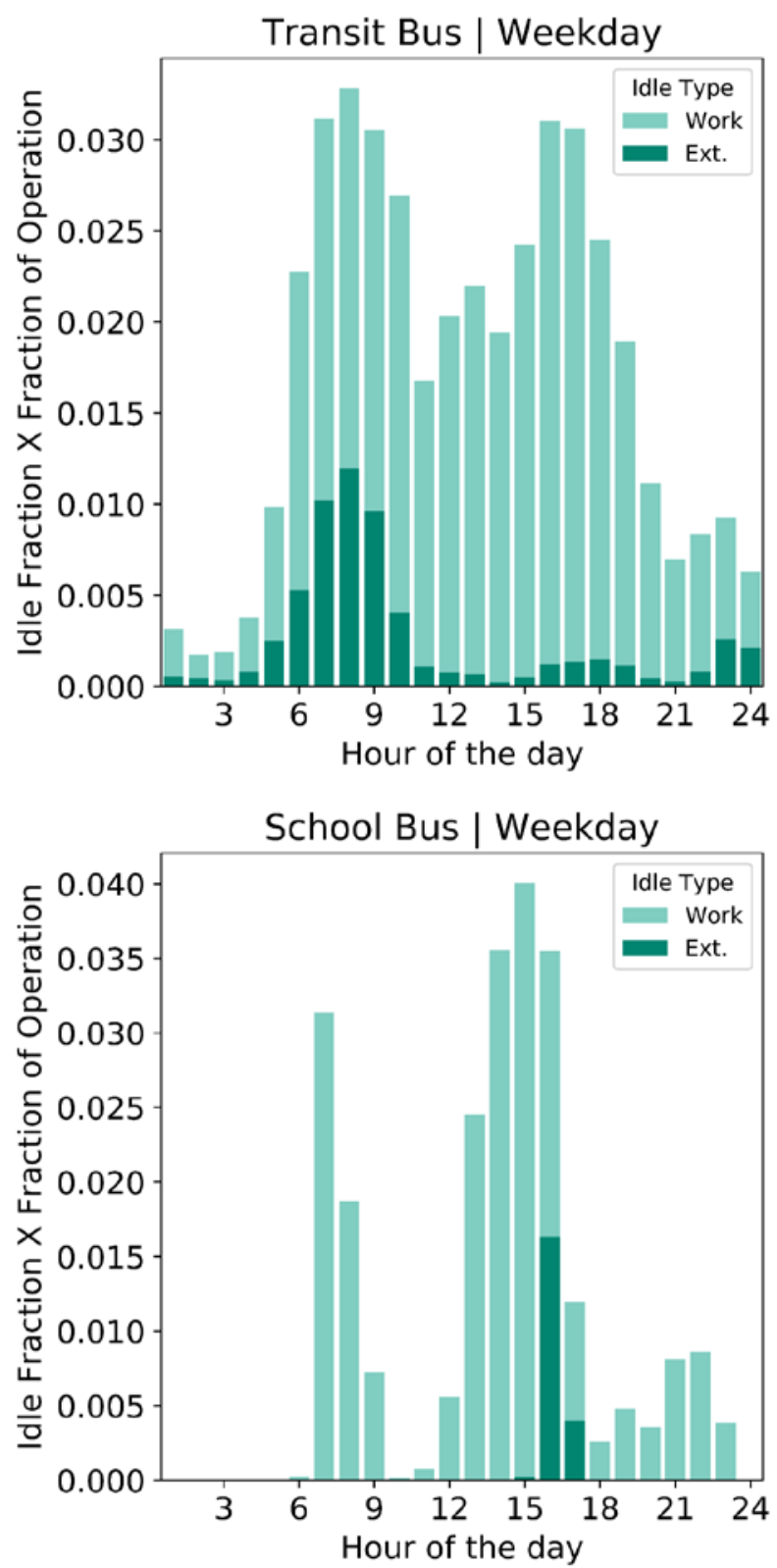

New
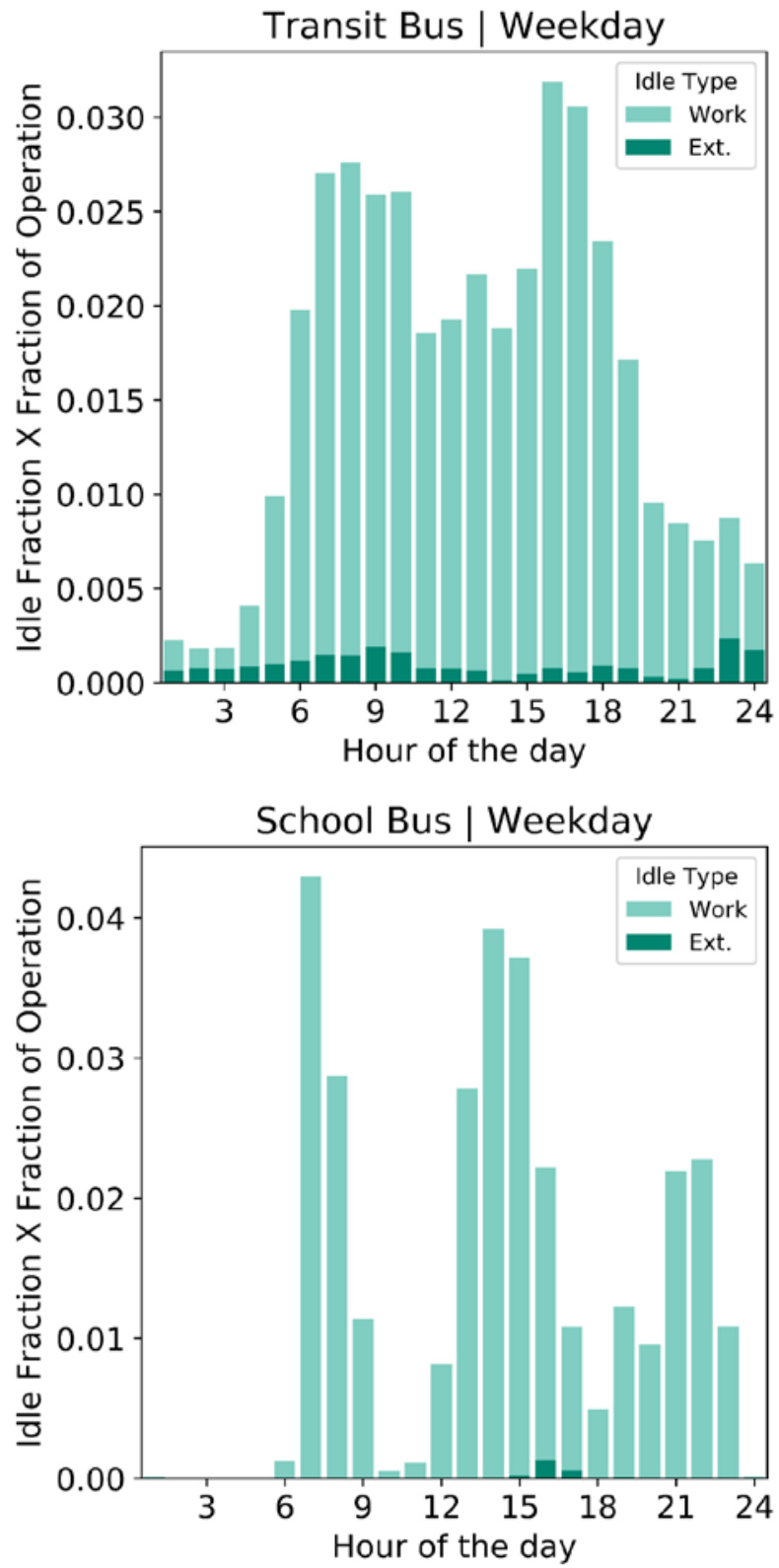
Old
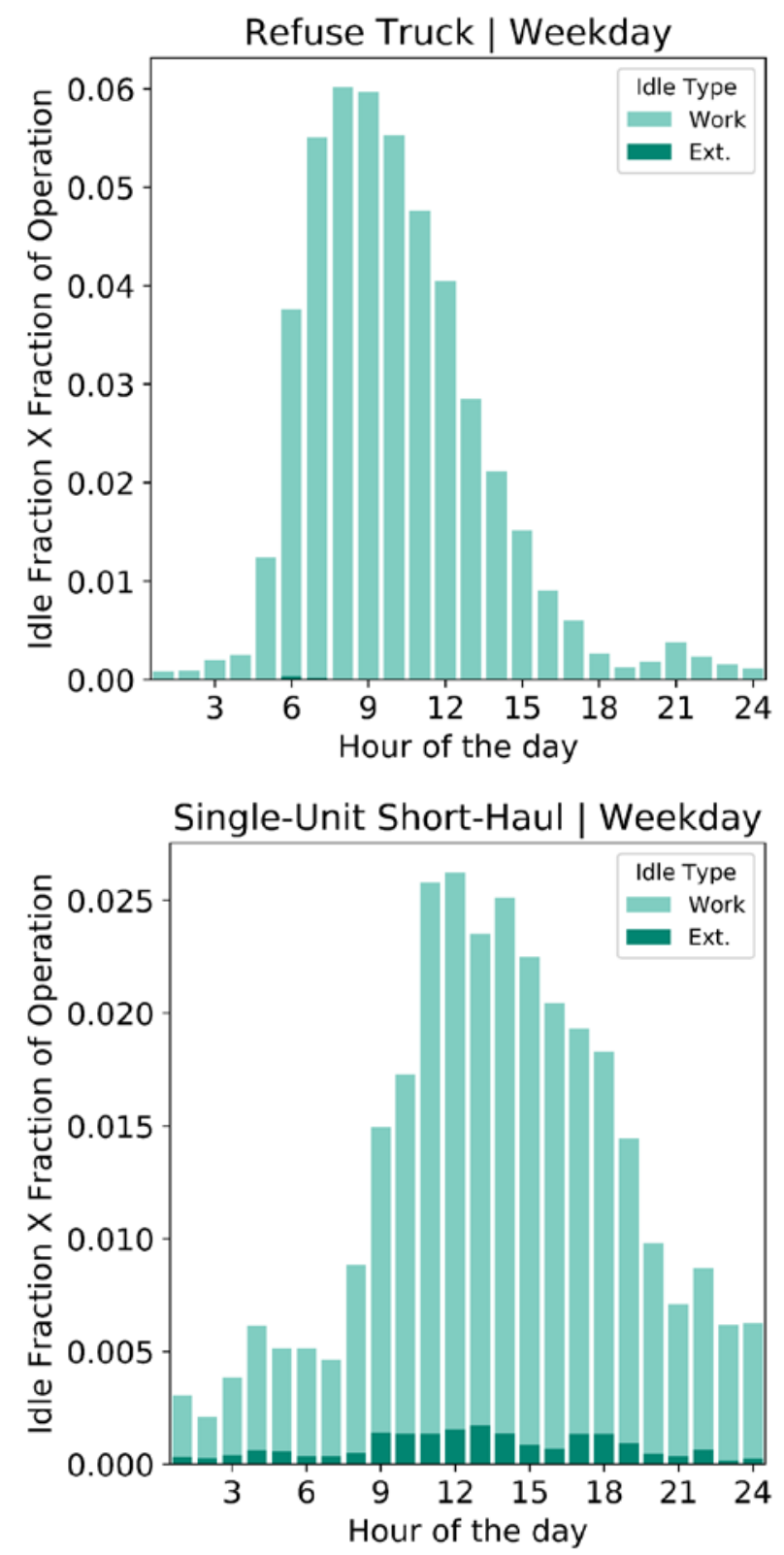

New
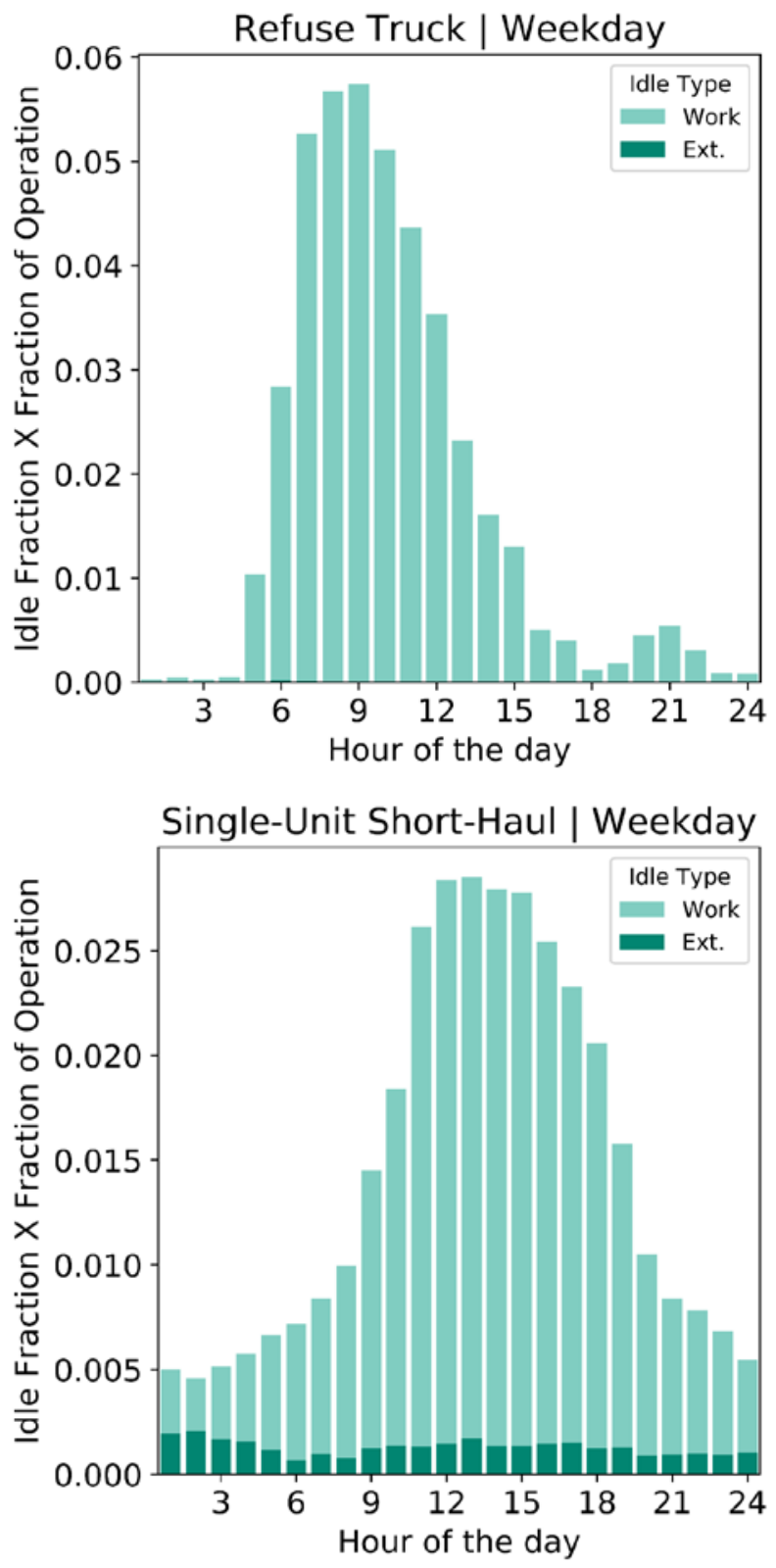
Old
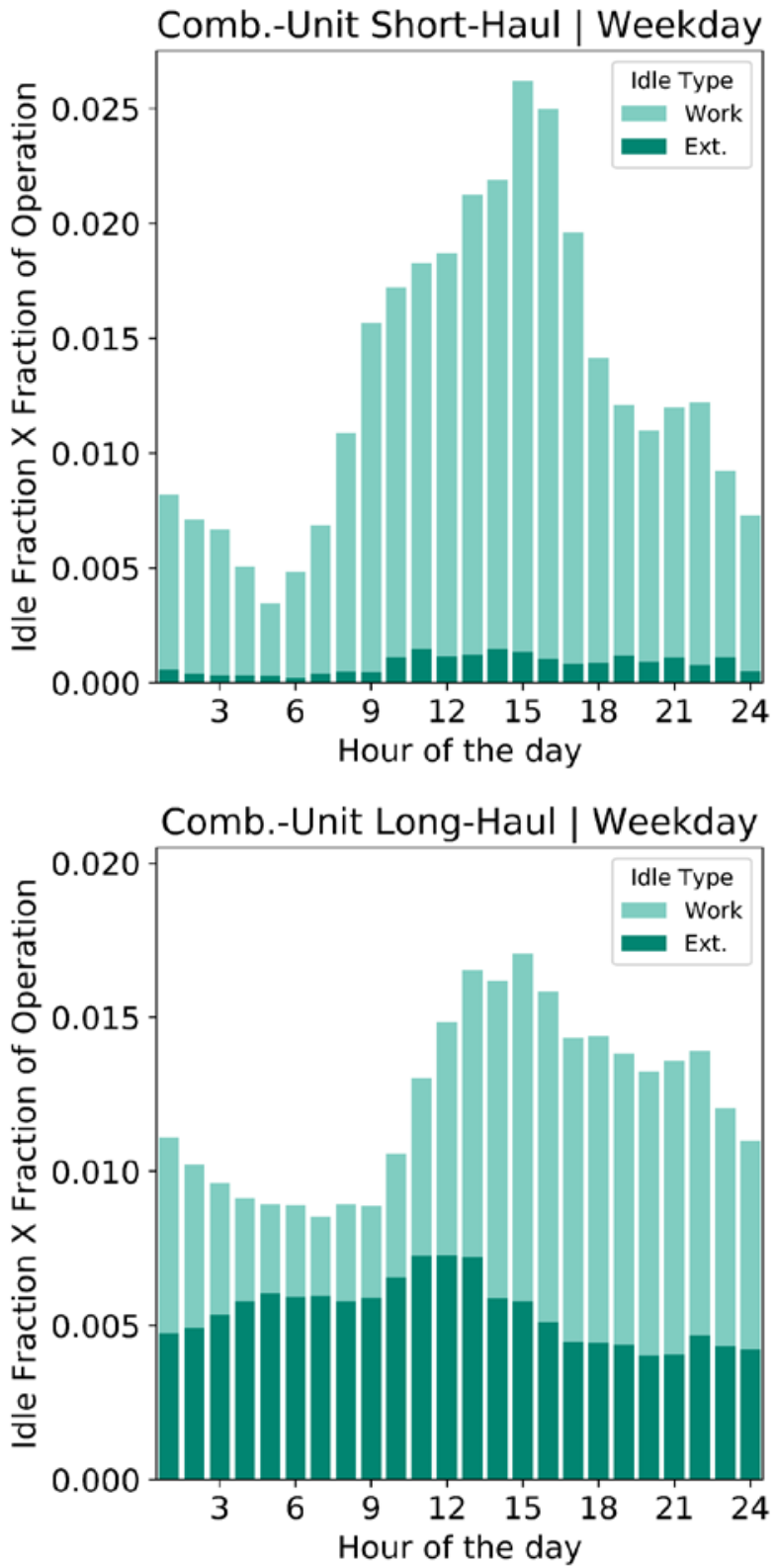

New
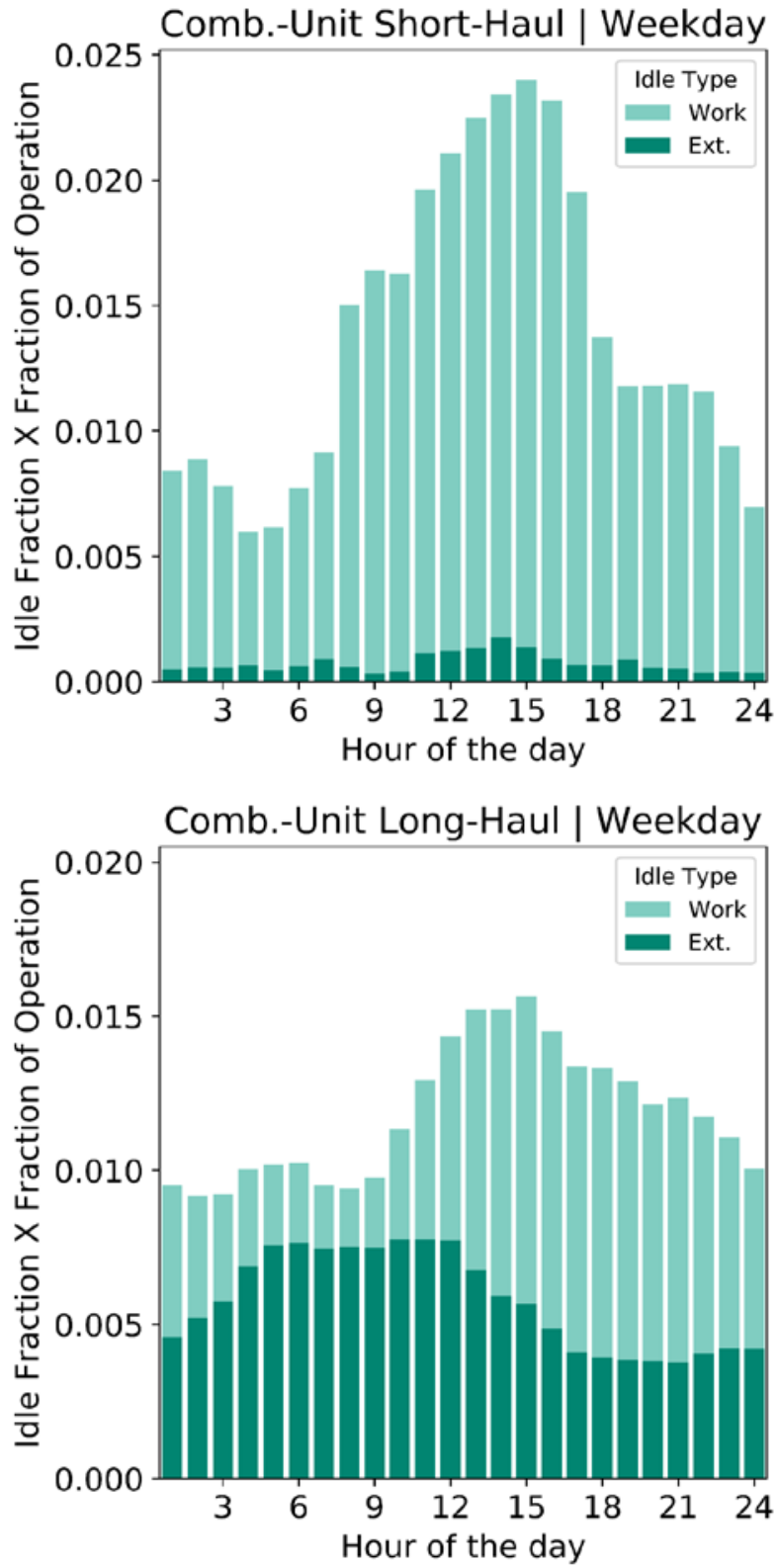